\title{
APPLICATION OF LATTICE GAS AUTOMATA TO ELECTROMAGNETIC SCATTERING AND TRANSMISSION LINE MODELLING
}

\author{
BY \\ LEILI SHAFAI
}

\begin{abstract}
A Thesis
Submitted to the Faculty of Graduate Studies

in Partial Fulfillment of the Requirements

for the Degree of
\end{abstract}

MASTER OF SCIENCE

Department of Electrical and Computer Engineering

University of Manitoba

Winnipeg, Manitoba, Canada

C Copyright by Leili Shafai, 2000 
National Library

of Canada

Acquisitions and Bibliographic Services

395 Wellington Street Ottawa ON KIA ONA Canada
Bibliothèque nationale du Canada

Acquisitions et services bibliographiques

395, nue Wellington

Ottawa ON KIA ONA

Canada
The author has granted a nonexclusive licence allowing the National Library of Canada to reproduce, loan, distribute or sell copies of this thesis in microform, paper or electronic formats.

The author retains ownership of the copyright in this thesis. Neither the thesis nor substantial extracts from it may be printed or otherwise reproduced without the author's permission.
L'auteur a accordé une licence non exclusive permettant à la Bibliothèque nationale du Canada de reproduire, prêter, distribuer ou vendre des copies de cette thèse sous la forme de microfiche/film, de reproduction sur papier ou sur format électronique.

L'auteur conserve la propriété du droit d'auteur qui protège cette thèse. $\mathrm{Ni}$ la thèse ni des extraits substantiels de celle-ci ne doivent être imprimés ou autrement reproduits sans son autorisation.

\section{Canadä}


THE UNIVERSITY OF MANITOBA

FACULTY OF GRADUATE STUDIES

COPYRIGHT PERMISSION PAGE

APPLICATION OF LATTICE GAS AUTOMATA TO ELECTROMAGNETIC

SCATTERING AND TRANSMISSION LINE MODELLING

BY

\title{
LEILI SHAFAI
}

A Thesis/Practicum submitted to the Faculty of Graduate Studies of The University of Manitoba in partial fulfillment of the requirements of the degree

of

\author{
MASTER OF SCIENCE
}

\section{LEILI SHAFAI O2000}

Permission has been granted to the Library of The University of Manitoba to lend or sell copies of this thesis/practicum, to the National Library of Canada to microfilm this thesis and to lend or sell copies of the film, and to Dissertations Abstracts International to publish an abstract of this thesis/practicum.

The author reserves other publication rights, and neither this thesis/practicum nor extensive extracts from it may be printed or otherwise reproduced without the author's written permission. 


\section{ABSTRACT}

Lattice gas automata consist of particles within a lattice that interact with their nearest neighbours according to simple deterministic rules. Essential parts of the algorithm can be implemented in a few lines of code, while stability is maintained by the exact conservation of mass and momentum within the system, with no round-off errors. This thesis deals with the application of lattice gas automata for use in modelling two-dimensional homogeneous electromagnetic phenomena. The behaviour of the HPP lattice gas automaton has been shown to model conditions described by the two-dimensional linear wave equation.

The application of the HPP-LGA algorithm to scattering experiments and transmission line modelling was explored in this thesis. The TLM method was used to assess the performance of the LGA data, as the implementation of the two algorithms in a a computer environment is similar. Furthermore, as an established computational technique, simulation results obtained using the TLM method represented an "exact" solution with which to evaluate the data obtained by the LGA algorithm. Properties inherent to twodimensional HPP-LGA are detailed through simple two-dimensional lattice gas experiments. These include the implementation of boundary conditions, system dissipation, and macroscopic averaging, as well as ensemble averaging of simulated data. 


\section{ACKNOWLEDGMENTS}

I would like to thank Professor G. Bridges for his supervision and guidance throughout the course of this thesis. Special thanks to Dino Cule for paving the way, and for his unsolicited assistance and advice.

I would also like to express my appreciation to Shelly Girardin and Dr. Cyrus Shafai for their assistance during times of crisis and frustration. I am also very grateful to my father, Dr. Lot Shafai, for his professional guidance in the dark hours. A special acknowledgment to both my parents for their encouragement and endless support through all my endeavors. And, last but not least, a very special thanks to Trevor Sawatzky, who has put up with all the long hours and late nights, and for his unfailing assistance and support from the beginning. Financial support from the Natural Sciences and Engineering Research Council of Canada and the University of Manitoba Duff Roblin Fellowship is gratefully acknowledged. 


\section{TABLE OF CONTENTS}

ABSTRACT ii

ACKNOWLEDGMENTS iii

TABLE OF CONTENTS iv

LIST OF FIGURES vii

LIST OF TABLES X xii

Chapter 1 INTRODUCTION 1

1.1 Cellular Automata 1

1.2 Lattice Gas Automata 2

1.3 Motivation 2

1.4 Scope 3

Chapter 2 LATTICE GAS AUTOMATA THEORY 5

2.1 HPP-LGA Algorithm 5

2.1.1 Collision 5

$\begin{array}{ll}2.1 .2 & \text { Advection }\end{array}$

$\begin{array}{lll}2.2 & \text { Microscopic Dynamics } & 10\end{array}$

$\begin{array}{lll}2.3 & \text { Macroscopic Behaviour } & 12\end{array}$

2.3.1 Linear Wave Behaviour 13

2.3.2 Macroscopic Observables 15

2.4 Similarities Between Fluid Flow and Electromagnetic Propagation 18

2.4.1 Analysis of Acoustic Waves at a Plane Boundary 19

2.4.2 Analysis of Electromagnetic Waves at a Plane Boundary 21

Chapter 3 ELEMENTARY LATTICE GAS EXPERIMENTS 25

$\begin{array}{lll}3.1 & \text { Source Excitation } & 26\end{array}$ 
3.2 Boundary Conditions

3.2.1 Perfectly Conducting Magnetic Boundary 29

3.2.3 Absorbing Boundary 34

3.3 Propagation Velocity and Viscosity 38

3.3.1 Propagation Velocity 38

3.3.2 Viscosity 39

3.4 Averaging 45

3.4.1 Spatial Averaging 45

3.4.2 Ensemble Averaging 47

3.5 Dimensional Translation 53

3.5.1 Spatial Averaging 56

3.5.2 Ensemble Averaging 58

3.6 Summary of Results $\quad 60$

Chapter 4 APPLICATION OF LGA TO SCATTERING PROBLEMS 61

4.1 Transmission Line Matrix Method 61

4.1.1 Lumped Element Model of Maxwell's Equations 61

4.1.2. TLM Algorithm 66

4.1.3 Boundary Conditions 68

4.1.3.1 Perfect Magnetic Conducting Boundary 68

4.1.3.2 Absorbing Boundary 69

4.2 Scattering Experiments $\quad 70$

4.2.1 Effect of Window Size on Scattered Signals 74

4.2.1.1 Time Domain 76

4.2.1.2 Frequency Domain 84

4.2.2 Effect of Cylinder Dimensions on Scattered Signals 95

4.2.2.1 Time Domain 96

4.2.2.2 Frequency Domain 104

4.2.3 Summary of Results 112 
Chapter 5 DESIGN OF A MICROWAVE TRANSMISSION LINE FILTER

5.1 Sample Microstrip Filter Design 113

5.2 Simulation of a Transmission Line Model 116

5.3 Simulation of a First Order Filter 122

5.3.1 Time Domain 122

5.3.1.1 LGA and TLM Configurations 122

5.3.1.2 Results 124

5.3.2 Frequency Domain 131

5.4 Summary of Results $\quad 135$

Chapter 6 CONCLUSIONS AND FUTURE RESEARCH 136

$\begin{array}{lll}6.1 & \text { Conclusions } & 136\end{array}$

$\begin{array}{lll}6.2 & \text { Future Research } & 139\end{array}$

Appendix COMPUTATIONAL EFFICIENCY 141

REFERENCES 143 


\section{List of Figures}

Figure 2.1: $\quad$ Particle streaming [8]. 6

Figure 2.2: $\quad$ Particle collision [8]. 6

Figure 2.3: Examples of HPP-LGA collision rules [7]. 7

Figure 2.4: Cell configuration. 8

Figure 2.5: Collision and advection over one time step $\Delta t[8]$. 9

Figure 2.6: Example of a square sampling window [7]. 16

$\begin{array}{ll}\text { Figure 2.7: } & 18\end{array}$

Figure 2.8: Reflection of an acoustic wave at a plane boundary. $\quad 20$

Figure 2.9: Reflection of an electromagnetic wave at a plane boundary. $\quad 21$

Figure 2.10: Reflection of an TE, wave at a plane boundary [8]. 23

Figure 3.1: Lattice configuration. 25

Figure 3.2: Gaussian pulsed plane wave excitation for $\rho_{m}=0.2,0.4,0.6$, and 0.8 ; (a) window 1, (b) window 2 , and (c) window 3 . 28

Figure 3.3: Perfect magnetic conducting boundary condition [8]. 29

Figure 3.4: Time-domain waveforms for four walled PMC mesh with $x_{o}=201 \Delta$; (a) window 1 ; (b) window 2; (c) window 3 . 31

Figure 3.5: Time-domain waveforms for PMC boundaries at north, south, east, and west walls, $x_{0}=1 \Delta x$; (a) window 1 ; (b) window 2 ;

(c) window 3.

Figure 3.6: Time-domain waveforms for north, south, and west PMC walls, $\mathrm{ABC}$ on east wall, $801 \Delta \times 801 \Delta$ mesh; (a) window 1;

(b) window 2 ; (c) window 3.

Figure 3.7: Time-domain waveforms for north, south, and west PMC walls, $A B C$ on east wall, $801 \Delta \times 801 \Delta$ mesh; (a) window l;

(b) window 2 ; (c) window 3 .

Figure 3.8: Lattice sound speed, $c_{s}$ vs. background distribution of particles, $\rho_{o} .40$

Figure 3.9: Computed decay, $\alpha$ vs. background distribution of particles, $\rho_{o} . \quad 42$

Figure 3.10: Time domain waveforms for $801 \Delta \mathrm{X} 401 \Delta$ mesh with four PMC walls; (a) $\rho_{o}=0.25$; (b) $\rho_{o}=0.35$; (c) $\rho_{o}=0.45$; (d) $\rho_{o}=0.55$. 
Figure 3.11: Time domain waveforms for $801 \Delta X 401 \Delta$ mesh with four PMC walls; window sizes (a); $80 \Delta \mathrm{X} \mathrm{80 \Delta ;} \mathrm{(b)} 120 \Delta \mathrm{X} 120 \Delta$;

(c) $160 \Delta \times 160 \Delta$.

Figure 3.12: Noise levels for $801 \Delta X 401 \Delta$ mesh PMC walls and $120 \Delta X$ $120 \Delta$ windows; (a) 1 run; (b) 5 runs; (c) 10 runs; (d) 20 runs.

Figure 3.13: Waveforms for $801 \Delta \times 401 \Delta$ mesh with PMC walls and I20 X X $120 \Delta$ windows; (a) 1 run; (b) 5 runs; (c) 10 runs; (d) 20 runs.

Figure 3.14: DFT of the incident pulse of Figure 3.4 (a) for a four walled PMC mesh with a Gaussian excitation launched at $x_{o}=1.0 \Delta$.

Figure 3.15: DFT of incident Gaussian for simulations of varying window size. 56

Figure 3.16: DFT of incident LGA Gaussian, theoretical Gaussian and sinc, and their product for windows sizes of (a) $80 \Delta \times 80 \Delta$, (b) $120 \Delta$ X $120 \Delta$, (c) $160 \Delta \times 160 \Delta$.

Figure 3.17: DFT of 1 simulation and 5,10 , and 20 simulation averages. $\quad 59$

$\begin{array}{lll}\text { Figure 4.1: } & \text { (a) Shunt node and (b) TLM equivalent [27]. } & 62\end{array}$

Figure 4.2: Schematic of a TLM mesh: (a) impulse excitation, (b) results of the first scattering event [28].

Figure 4.3: Node configuration.

Figure 4.4: (a) LGA lattice, (b) TLM lattice, (c) physical cavity dimensions, with rectangular cylinder cross-section and two sampling windows.

Figure 4.5: Mathematical model for calculating the equivalent circular diameter.

Figure 4.6: Time domain waveforms of window 1 without scattering object for window dimensions of (a) $80 \Delta \times 80 \Delta$, (b) $120 \Delta \times 120 \Delta$, and (c) $160 \Delta \times 160 \Delta$.

Figure 4.7: Time domain waveforms of window 2 without scattering object for window dimensions of (a) $80 \Delta \times 80 \Delta$, (b) $120 \Delta \times 120 \Delta$, and (c) $160 \Delta \times 160 \Delta$.

Figure 4.8: Time domain waveforms of window 1 for $60 \Delta \times 120 \Delta$ cylinder cross-sectional area computed with window dimensions of (a) $80 \Delta \times 80 \Delta$, (b) $120 \Delta \times 120 \Delta$, and (c) $160 \Delta \times 160 \Delta$.

Figure 4.9: Time domain waveforms of window 2 for $60 \Delta$ X 120 cylinder cross-sectional area computed with window dimensions of 
(a) $80 \Delta \times 80 \Delta$, (b) $120 \Delta \times 120 \Delta$, and (c) $160 \Delta \times 160 \Delta$.

Figure 4.10: Backward scatter at window 1 for $60 \Delta \times 120 \Delta$ cylinder crosssectional area computed in the time domain with window dimensions of (a) $80 \Delta \times 80 \Delta$, (b) $120 \Delta \times 120 \Delta$, and

(c) $160 \Delta \times 160 \Delta$.

Figure 4.11: Forward scatter at window 2 for $60 \Delta \times 120 \Delta$ cylinder crosssectional area computed in the time domain with window dimensions of (a) $80 \Delta \times 80 \Delta$, (b) $120 \Delta \times 120 \Delta$, and (c) $160 \Delta \times 160 \Delta$.

Figure 4.12: Normalized Fourier transform of a rectangular gate and a circular gate function of (a) identical widths, and (b) with equivalent widths.

Figure 4.13: Frequency response of the incident pulse computed with window dimensions of (a) $80 \Delta \times 80 \Delta$, (b) $120 \Delta \times 120 \Delta$, and (c) $160 \Delta \times 160 \Delta$.

Figure 4.14: Frequency response of window 1 for $60 \Delta \times 120 \Delta$ cylinder crosssectional area computed with window dimensions of

(a) $80 \Delta \times 80 \Delta$, (b) $120 \Delta \times 120 \Delta$, and (c) $160 \Delta \times 160 \Delta$.

Figure 4.15: Frequency response of window 2 for $60 \Delta \times 120 \Delta$ cylinder crosssectional area computed with window dimensions of (a) $80 \Delta \times 80 \Delta$, (b) $120 \Delta \times 120 \Delta$, and (c) $160 \Delta \times 160 \Delta$.

Figure 4.16: DFT of backward scatter response for $60 \Delta \times 120 \Delta$ cylinder crosssectional area computed with window dimensions of

(a) $80 \Delta \times 80 \Delta$, (b) $120 \Delta \times 120 \Delta$, and (c) $160 \Delta \times 160 \Delta$.

Figure 4.17: DFT of forward scatter response for $60 \Delta \times 120 \Delta$ cylinder crosssectional area computed with window dimensions of (a) $80 \Delta \times 80 \Delta$, (b) $120 \Delta \times 120 \Delta$, and (c) $160 \Delta \times 160 \Delta$.

Figure 4.18: Response of the backward scatter to the incident pulse for $60 \Delta \times 120 \Delta$ cylinder cross-sectional area with window dimensions of (a) $80 \Delta \times 80 \Delta$, (b) $120 \Delta \times 120 \Delta$, and (c) $160 \Delta \times 160 \Delta$.

Figure 4.19: Response of the forward scatter to the incident pulse for $60 \Delta \times 120 \Delta$ cylinder cross-sectional area with window dimensions of (a) $80 \Delta \times 80 \Delta$, (b) $120 \Delta \times 120 \Delta$, and (c) $160 \Delta \times 160 \Delta$.

Figure 4.20: Lattice configuration with rectangular cylinders of varying cross-sectional areas.

Figure 4.21: Total field (incident and backward scatter) waveforms of window 1 for (a) no object, and for a cylinder cross-sectional 
area of (b) $60 \Delta \times 120 \Delta$, (c) $140 \Delta \times 120 \Delta$, and (d) $60 \Delta \times 240 \Delta$.

Figure 4.22: Total field (incident and forward scatter) waveforms of window 2 for (a) no object, and for a cylinder cross-sectional area of (b) $60 \Delta \times 120 \Delta$, (c) $140 \Delta \times 120 \Delta$, and (d) $60 \Delta \times 240 \Delta$.

Figure 4.23: Time domain waveforms of backward scatter in window $l$ for cylinder cross-sectional area of (a) $60 \Delta \times 120 \Delta$, (b) $140 \Delta \times 120 \Delta$, and (c) $60 \Delta \times 240 \Delta$.

Figure 4.24: Time domain waveforms of forward scatter in window 2 for cylinder cross-sectional area of (a) $60 \Delta \times 120 \Delta$, (b) $140 \Delta \times 120 \Delta$, and (c) $60 \Delta \times 240 \Delta$.

Figure 4.25: Frequency response (incident and backward scatter) in window 1 for a cylinder cross-sectional area of (a) $60 \Delta$ X 120 (b) $140 \Delta \times 120 \Delta$, and (c) $60 \Delta \times 240 \Delta$.

Figure 4.26: Frequency response (incident and forward scatter) of window 2 for a cylinder cross-sectional area of (a) $60 \Delta \times 120 \Delta$, (b) $140 \Delta \times 120 \Delta$, and (c) $60 \Delta \times 240 \Delta$.

Figure 4.27: Frequency response of backward scatter in window 1 for a cylinder cross-sectional area of (a) $60 \Delta \times 120 \Delta$,

(b) $140 \Delta \times 120 \Delta$, and (c) $60 \Delta \times 240 \Delta$.

Figure 4.28: Frequency response of forward scatter in window 2 for a cylinder cross-sectional area of (a) $60 \Delta \times 120 \Delta$,

(b) $140 \Delta \times 120 \Delta$, and (c) $60 \Delta \times 240 \Delta$.

Figure 4.29: Response of the backward scatter in window 1 to the incident pulse for cylinder cross-sectional area of (a) $60 \Delta \times 120 \Delta$, (b) $140 \Delta \times 120 \Delta$, and (c) $60 \Delta \times 240 \Delta$.

Figure 4.30: Response of the forward scatter in window 2 to the incident pulse for cylinder cross-sectional area of (a) $60 \Delta \times 120 \Delta$,

(b) $140 \Delta \times 120 \Delta$, and (c) $60 \Delta \times 240 \Delta$.

Figure 5.1: Filter network structure.

Figure 5.2: Single stub transmission line filter design. 114

Figure 5.3: Simulation of transmission line filter using Libra. 115

Figure 5.4: Transmission line configuration for LGA simulation. 116

Figure 5.5: Time domain waveforms for a single transmission line with rectangular window dimensions of (a) $50 \times 50$, (b) $60 \times 50$, (c) $80 \times 50$, (d) $100 \times 50$, (e) $120 \times 50$, and (f) $140 \times 50$.

Figure 5.6: Representation of the input Gaussian signal, the actual pulse 
observed by the $120 \Delta X 50 \Delta$ window, and the theoretical rms noise level.

Figure 5.7: Time-domain output of a 10 run ensemble average for a

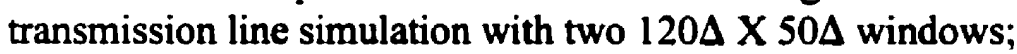
(a) window 1 , (b) window 2.

Figure 5.8: Filter configuration for LGA simulation. 122

Figure 5.9: Filter configuration for TLM simulation. 123

Figure 5.10: Time domain waveforms for (a) input (window 1), (b) output (window 2), and (c) the stub window, for the simulated TLM and LGA filter.

Figure 5.11: Reflected and transmitted power in AB for LGA and TLM frequency transmission line filter; (a) $S_{11}$, and (b) $S_{21}$.

Figure 5.12: Reflected and transmitted power in $\mathrm{dB}$ of four individual LGA seed simulations; (a) $S_{11}$, and (b) $S_{21}$. 


\section{List of Tables}

Table 3.1: Time at each observation point, their spatial distance, and sound speed in each sampling window.

Table 3.2: Propagation velocities for $\rho_{o}=0.25$ to 0.60 . 40

Table 3.3: Computed decay, $\alpha$, for $\rho_{o}=0.25$ to 0.60 . 42

Table 3.4: Rms noise levels for single, 5,10 , and 20 ensemble simulations. $\quad 47$

Table 5.1: Computed and predicted pulse amplitudes in $\mathrm{V} / \mathrm{m}$ at windows 1,2 , and 3 (input, output, and stub) for the first-order mismatch.

Table 5.2: $\quad$ Observed and ideal pulse amplitudes in $\mathrm{V} / \mathrm{m}$ at windows 1,2 , and 3 (input, output, and stub) for the second and third order mismatches.

Table 5.3: Amplitudes in $\mathrm{V} / \mathrm{m}$ of the $\mathrm{ABC}$ reflections at windows 1 and 2 (input and output) introduced after the first $\mathrm{ABC}$ reflection, and their resulting mismatch at the stub junction.

Table 5.4: Amplitudes in $\mathrm{V} / \mathrm{m}$ of the $\mathrm{ABC}$ reflections at windows 1 and 2 (input and output) introduced after the second $A B C$ reflection, and their resulting mismatch at the stub junction.

Table 5.5: Correction of reflected and transmitted signal by contribution of the first $A B C$ reflections in $\mathrm{V} / \mathrm{m}$ at the three port windows.

Table 5.6: Correction of reflected and transmitted signal by contribution of the second $A B C$ reflections in $\mathrm{V} / \mathrm{m}$ at the three port windows.

Table A.1: Number of operations and CPU time completed for selected experiments on Unix Ultra 5 and Ultra 10 stations. 


\section{Chapter 1}

\section{INTRODUCTION}

\subsection{Cellular Automata}

Cellular automata are discrete dynamical systems capable of modelling physical phenomena. The cellular automata system has a spatially discrete geometry consisting of a large lattice of identical cells. Each cell within the lattice contains a small number of states represented by a few bits of data. Evolution of the system is governed by simple rules that are local and uniform in space and time. With each discrete time step, the data within each cell is computed according to the data located in the cells of its nearest neighbours.

Mathematician and physicist John von Neumann introduced the concept of cellular automata in the late 1940's. The initiative was proposed originally by Stan Ulam, "to provide a more realistic model for the behaviour of complex extended systems" [1]. Von Neumann used the concept of cellular automata for the purpose of modelling certain biological systems. Over the years, cellular automata has been studied for the application of physical laws. Wolfram, in the early 1980's, showed their capability of exhibiting complex dynamical behaviour [2,3], through experimentation with one-dimensional cellular automata. Further investigations have been found to provide accurate models of differential equations (heat and wave) $[1.4]$, as well as the Navier-Stokes equation of fluid dynamics $[1,5,6]$. Several branches of study have emerged, where cellular automata are being used to model such physical phenomena as diffusion, turbulence, semi-conductor device modelling and electromagnetics [7]. 


\subsection{Lattice Gas Automata}

Lattice gas automata (LGA), a class of cellular automata, consist of particles within a lattice that interact with their nearest neighbours according to simple deterministic rules. Cellular automata evolve through a series of generations, proceeding from generation to generation in single steps. In contrast, LGA evolve through a two step process [8] of collision and advection similar to a fluid.

Lattice gas techniques have been used to successfully simulate the Navier-Stokes equation. and thus have been shown to simulate fluid flow. However, instead of explicitly solving the Navier-Stokes equations, the movements and interactions of thousands of gas particles are monitored [9]. The rules dictate the state of each cell, and conserve the mass and momentum of the particles within each cell.

Similarities between fluid flow and wave propagation suggest that LGA can be used to simulate electromagnetic models. The idea of LGA as a new and "innovative" [9] method of electromagnetic analysis was proposed in the late 1980's. Initial tests with simple twodimensional electromagnetic phenomena are presented in [10], and with further detail in [2.7.8.11], where comparisons to FDTD, and in particular, TLM methods are addressed. The TLM and LGA techniques share similar properties, and as such, the TLM method has been used to compare and analyze LGA results with an established computational technique.

\subsection{Motivation}

Simplicity, stability, and the ability to model complicated boundaries are important features of LGA techniques. Essential parts of the algorithm can be implemented in a few lines of code, while stability is maintained by the exact conservation of mass and momentum within the system, with no round-off errors. Additionally, boundary conditions are easy to implement and can be simulated within a structure of significant complexity over a large 
number of time steps [12].

An important advantage of LGA methods is their memory efficiency. Unlike traditional differential equation based computation approaches, such as Finite-Difference Time-Domain (FDTD), or Transmission-Line Matrix (TLM) methods, which use floating point variable based techniques, LGA use only simple binary terms (I's and 0's) to describe the information in each cell. Therefore, LGA techniques require only a fraction of the bits (usually from 4 to 6, depending on the algorithm) to completely define the contents of a cell, while standard differential equation based calculations are computed using 64 bit words, regardless of the size of the variables. Memory storage is thus more efficient. Further, the rules governing particle interaction can be implemented by table look-up, or by logic operation. Consequently, since their operations are bit-oriented, LGA methods execute more naturally in a computer environment. The lattice gas automaton is thus exactly computable, resulting in a faster computation time, with no loss of information due to round-off error.

LGA algorithms are inherently parallel and thus evolve more efficiently on a parallel machine. rather than a conventional sequential computer. Parallel computing systems have been specifically designed for the simulation of LGA systems and are known as cellular automata machines (CAM). Its simulation speed is several orders of magnitude faster than a conventional computer. Further information regarding the CAM environment is detailed in $[1,13]$.

\subsection{Scope}

This thesis deals with the application of lattice gas automata for use in modelling two-dimensional electromagnetic phenomena. Only homogeneous structures are considered, and thus discussion concerning the modelling of inhomogeneities will not be covered.

Chapter 2 presents the theory and rules that govern a lattice gas system. The microscopic and macroscopic behaviour of the LGA system are detailed, with emphasis on the linear wave behaviour with regard to the approximation of the Navier-Stokes equation. 
Similarities are highlighted for acoustic and electromagnetic wave analysis at a plane boundary.

In Chapter 3, properties inherent to two-dimensional lattice gas automata are detailed using results from simple two-dimensional lattice gas experiments. These include discussions concerning the effects of viscosity, averaging, dimensional translation, i.e. time domain vs. frequency domain, as well as propagation velocity, source excitation, and boundary conditions.

In Chapter 4, lattice gas techniques are applied to the analysis of plane wave scattering from a perfectly conducting cylinder. Three different cylinder geometries, as well as three variations of the mesh configuration, were simulated, with time domain and trequency domain results compared with traces obtained using the TLM method.

Chapter 5 details simple transmission line experiments by LGA, with a progression to transmission line/microstrip filter designs. Again, analysis of the time and frequency domain is provided with a comparison of results obtained using the TLM method.

Finally, a conclusion is presented with a summary of the information presented in this thesis and with recommendations for future study. 


\section{Chapter 2}

\section{LATTICE GAS AUTOMATA THEORY}

\subsection{HPP-LGA Algorithm}

The class of cellular automaton of interest in this thesis is the HPP lattice gas model. The HPP acronym refers to the model's three inventors, J. Hardy, O de. Pazzis, and Y. Pomeau, who originally introduced the system in 1973 as a study of "a particularly simple two-dimensional system of classical particles" [14]. The algorithm was further detailed with specific reference to the dynamics of the Navier-Stokes equation of hydrodynamics in a 1976 paper [5] by the same authors.

\subsubsection{Collision}

The HPP-LGA model consists of a two-dimensional Cartesian lattice. Each cell is restricted to a maximum of four moving particles, with each particle occupying a discrete site within a cell. Movement of each particle is limited to the north, south, east, and west directions of the cell, with no more than one moving particle in each of the four directions, a property known as the exclusion principle.

In one time step, a particle will advance one step to the next cell in its direction of motion, as shown in Figure 2.1. In Figure 2.1 (b), the east going particle moves to the neighbouring right cell and the west going particle moves on to the left neighbouring cell without any interaction. The northbound cell simply continues in its northbound direction. They continue along this path, unless they collide head-on with another particle traveling in the opposite direction. A head-on collision results in a transformation of velocity and the 
two particles are scattered at right angles to their original velocity state, detailed in Figure 2.2. Particles in other configurations, i.e. cells having one, three, and four particles, or two particles at right angles to each other, experience no transformation of velocity. They simply pass through one another as they continue along their original direction of motion. The HPP collision rules, summarized in Figure 2.3, are rotationally symmetric. That is, the rules are unchanged when the input particle configurations are rotated by $90^{\circ}$.

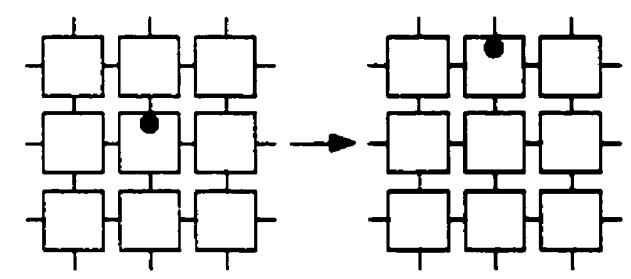

(a)

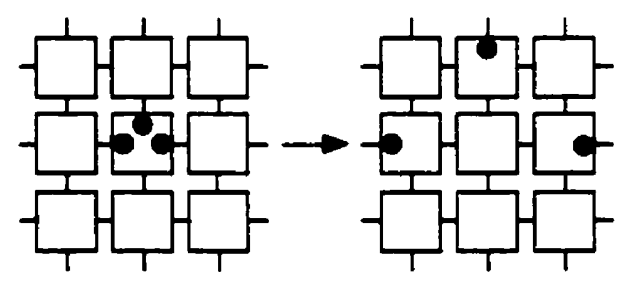

(b)

Figure 2.1: Particle streaming [8].

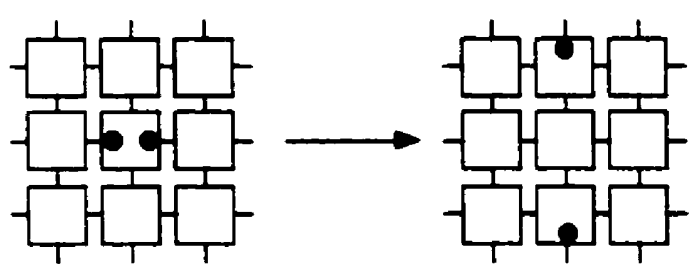

Figure 2.2: Particle collision [8]. 


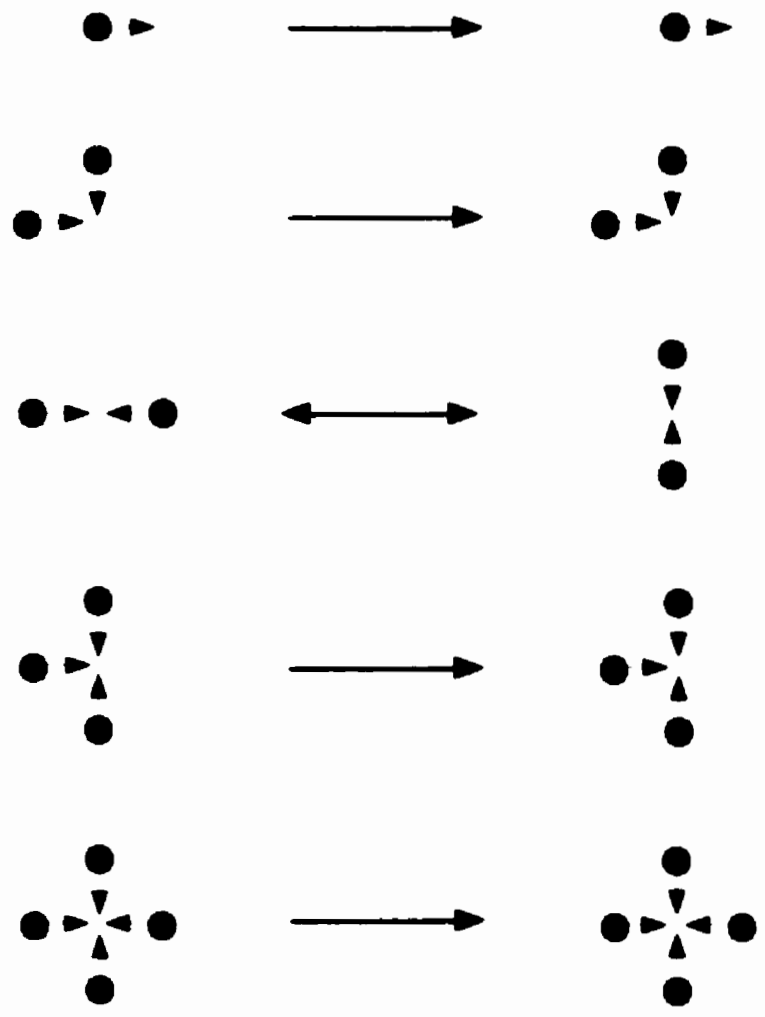

Figure 2.3: Examples of HPP-LGA collision rules [7]. 
Binary notation is used to denote the state of a particle site, where a "l" represents a site occupied by a particle, and a " 0 " represents a hole. The particle configuration within the lattice is defined by a cell $E[5]$,

$$
E_{i j}=d_{i j} c_{i j} b_{i j} a_{i j}
$$

where $d, c, b$, and $a$ represent the four sites (west, north, east, and south, shown in Figure 2.4) of a particular lattice cell with $i$ and $j$ denoting the column and row indices of the lattice. The state of each cell is therefore represented by four bits. Thus. there are a total of sixteen $\left(4^{2}\right)$ possible cell states,

$$
0 \leq E_{i j} \leq 1111
$$

and the effect of a collision on the matrix $E$ may be expressed as,

$$
\hat{C}\left(E_{i j}\right)=\left\{\begin{array}{cc}
0101, & \text { if } E_{i j}=1010 \\
1010, & \text { if } E_{i j}=0101 \\
E_{i j}, & \text { otherwise }
\end{array}\right.
$$

where $\hat{C}$ is the collision operator. The transition of velocity states resulting from a collision within a cell, is easily accomplished with the use of a look-up table.

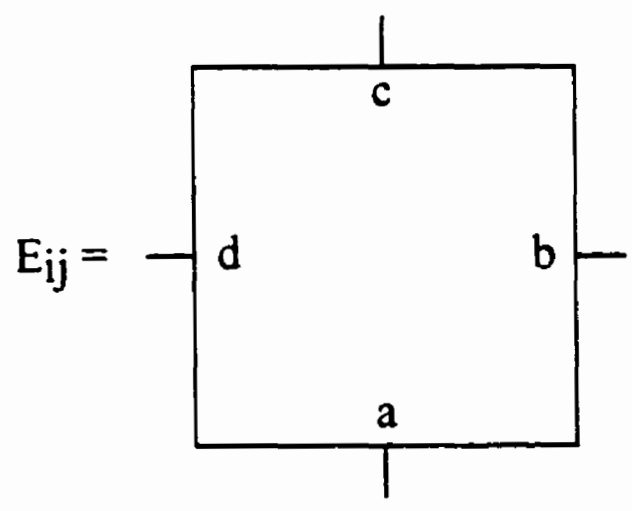

Figure 2.4: Cell configuration. 


\subsubsection{Advection}

In the HPP-LGA algorithm, one time step consists of two distinct stages: (1) a collision stage, and (2) an advection stage. In the collision stage, there is no interaction between cells, i.e. the mass and momentum of an individual cell remain unchanged, but the velocity states of each particle may differ according to the HPP collision rules and their original configurations. The advection stage involves a "free translation" [5], with no change in velocities. Particles are transferred to neighbouring cells following the collision event, shown in Figure 2.5. Referring to the notation used in the previous section, the advection can be described by a translation operator $T_{o}[5]$,

$$
\left(T_{o} E\right)_{i j}=d_{i-1 j} c_{i j-1} b_{i-1, j} a_{i j-1}
$$

to denote that each particle takes one step forward in the direction of its velocity.

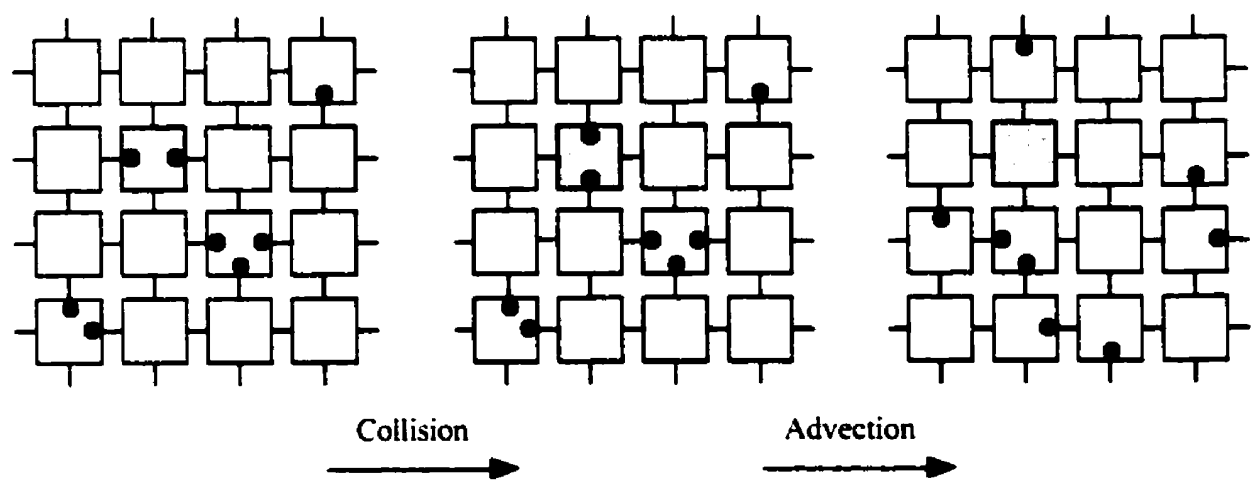

Figure 2.5: Collision and advection over one time step $\Delta t$ [8]. 


\subsection{Microscopic Dynamics}

A mathematical description of the microscopic dynamics of a lattice gas $[7,8]$, outlined in section 2.1 , is presented in this section. The velocity states, $c$, to which the particles of a particular cell may exist can be expressed by,

$$
c^{j}=\cos \left[\frac{\pi}{2}(j-1)\right] \hat{x}+\sin \left[\frac{\pi}{2}(j-1)\right] \hat{y}, \quad j=1,2,3,4
$$

Let $b_{i}(x, t)$ represent the presence or absence $(1$ or 0$)$ of a particle at time $t$, in velocity state $i$, of lattice site $x$. At the next time step, $t+\Delta t$, the expression for particles that do not experience a transformation of velocity (Figure 2.1 ) is written as,

$$
b_{i}\left(x+c^{i} \Delta l, t+\Delta t\right)=b_{i}(x, t)
$$

Thus, updating using (2.6) applies to a lattice with no collision, involving only particle streaming.

For a lattice with particles that undergo a collision (Figure 2.2), the expression becomes,

$$
b_{i}\left(x+c^{i} \Delta l, t-\Delta t\right)=b_{i}(x, t)-C_{i}(b(x, t))
$$

where $b(x, t)$ is a vector representing all the bits in cell $\boldsymbol{x}$ at time $t$, and $C_{t}(\boldsymbol{b}(\boldsymbol{x}, t)$ is known as the microscopic collision operator for direction $i$ which has $b(x, t)$ as its input. Therefore, the presence or absence of a particle in velocity state $i$ of cell $x+c_{i} \Delta l$ at time $t+\Delta t$ is known.

The complete dynamics of the HPP-LGA can now be described by a set of boolean equations, where the subscript $i$ has been replaced by $N, S, E, W$, representing the north, south, east, and west states, respectively, of cell $x$. The symbol $\bar{b}_{i}$ represents the complement of bit $b_{i}$, where $b_{i}$ is used for $b_{i}(x, t)$ to simplify the notation. 


$$
\begin{aligned}
& b_{N}(x, y+\Delta l, t+\Delta t)=b_{N}+C_{N} \\
& =b_{N}+\overline{b_{N} b_{S}} b_{E} b_{W}-b_{v} b_{S} \overline{b_{E} b_{W}} \\
& b_{s}(x, y-\Delta l, t+\Delta t)=b_{s}+C_{s} \\
& =b_{S}+\overline{b_{v} b_{S}} b_{E} b_{W}-b_{v} b_{S} \overline{b_{E} b_{W}} \\
& b_{E}(x+\Delta l, y, t+\Delta t)=b_{E}+C_{E} \\
& =b_{E}+b_{v} b_{s} \overline{b_{E} b_{W}}-\overline{b_{v} b_{s}} b_{E} b_{w} \\
& b_{w}(x-\Delta l, y, t+\Delta t)=b_{w}-C_{W} \\
& =b_{W}+b_{v} b_{s} \overline{b_{E} b_{W}}-\overline{b_{v} b_{s}} b_{E} b_{W}
\end{aligned}
$$

The total number of particles (mass), and the momentum along the $x$ - and $y$-axis of the lattice are conserved in the HPP-LGA model. Collisions conserve mass and momentum locally. Advection passes information throughout the lattice conserving them globally [15]. This can be shown mathematically using (2.8) to (2.11), for the conservation of mass (2.12), and for the conservation of momentum in the $x(2.13)$ and $y(2.14)$ directions, respectively.

$$
\begin{gathered}
C_{\mathrm{v}}+C_{S}+C_{\mathrm{e}}-C_{W}=0 \\
C_{E}-C_{W}=0 \\
C_{\mathrm{N}}-C_{S}=0
\end{gathered}
$$




\subsection{Macroscopic Behaviour}

The creators of the HPP-LGA model focused on the Navier-Stokes hydrodynamical equation to explore the dynamics of lattice gases. As a result, the area of fluid dynamics has undergone significant study with respect to modelling phenomena using lattice gases. However, the HPP-LGA algorithm was found to only approximate the Navier-Stokes equation. The HPP automaton is unable to provide the correct viscosity when modelling the complete equation [16]. This limitation was overcome by the FHP-LGA model [17] (inventors Frisch, Hasslacher, and Pomeau), a hexagonal lattice which correctly models the complete equation, including the viscosity. To correctly model the viscosity, lattice vectors must be isotropic to the fourth rank $[3,6,7]$. The symmetry of the HPP lattice is insufficient to insure tho isotropy of the fourth-degree tensor relating momentum flux to quadratic terms in the momentum equation [6]. The limitations of the HPP-LGA model in representing the Navier-Stokes equation can be neglected by considering only fluid flow involving small perturbations from an equilibrium state (a uniform background distribution of particles with no initial flow) with low fluid viscosity $[2,17,1]$. This limited model, although not accurate for simulating the full Navier-Stokes equation, is adequate for simulation of linear wave equations by the HPP-LGA model. The propagation of a disturbance or "wave" is permitted by the deletion of the non-linear and viscous terms $[18,1]$. Conservation of mass and momentum is also satisfied by this method.

The collision and advection rules detailed in section 2.1, outline the microscopic behaviour of the HPP-LGA algorithm. However, it is at the macroscopic level where the wave equations and the Navier-Stokes equation have been reproduced. The relationship between the lattice gas macroscopic behaviour and the Navier-Stokes equation is due to the conservation of mass and momentum within the lattice. Specifically, the particle number (mass) and impulsion (momentum) are conserved during the collision and moving of particles [18]. 


\subsubsection{Linear Wave Behaviour}

The modelling of a fluid is expressed by the two-dimensional Navier-Stokes equation and the continuity equation, respectively $[11,16,19]$,

$$
\begin{gathered}
\frac{\partial}{\partial t} \rho \boldsymbol{u}-\rho(\boldsymbol{u} \cdot \nabla) \boldsymbol{u}=-\nabla p-\mu \nabla^{2} \boldsymbol{u} \\
\frac{\partial \rho}{\partial t}-\nabla \cdot(\rho \boldsymbol{u})=0
\end{gathered}
$$

where $\rho$ is the density, $p$ the pressure, $u$ the velocity, and $\mu$ is the shear viscosity of the fluid. For small perturbations from a uniform background distribution with low velocity, the nonlinear and viscous effects of the fluid can be neglected. Under these conditions of uniform flow, Euler's equation results, where the conservation of momentum within the fluid can be expressed as [2],

$$
\frac{\partial}{\partial t} \rho u=\rho \frac{\partial u}{\partial t}=-\nabla p
$$

and the conservation of mass becomes,

$$
\nabla \cdot(\rho u) \approx \rho \nabla \cdot u=-\frac{\partial \rho}{\partial t}
$$

Fluid density, pressure, and flow can be expressed as the sum of their of uniform background distribution and small perturbations parts $[2,19,20]$,

$$
\begin{aligned}
& \rho=\rho_{o}-\rho_{e} \\
& p=p_{o}-p_{e} \\
& u=u_{e}
\end{aligned}
$$


where $\rho_{o}$ and $p_{o}$ are the uniform background density and pressure, and $\rho_{e}, p_{e}, u_{e}$ are the small density, pressure and flow perturbations, respectively. Considering the small density and flow velocity perturbations, a consistent approximation to the conservation of momentum in (2.17) using the quantities in (2.19) is [11,20],

$$
\frac{\partial u_{e}}{\partial t}=-\frac{1}{\rho_{o}} \nabla p_{e}
$$

The conservation of mass becomes [9],

$$
\rho_{o} \nabla \cdot u_{e}=-\frac{\partial \rho_{e}}{\partial t}
$$

by neglecting $\rho_{e} u_{e}$ as a second order quantity [19].

For small $\boldsymbol{u}$ (compared to individual particle velocity), density and pressure are related as follows $[8,11]$,

$$
c_{s}=\left(\frac{\partial p}{\partial \rho}\right)_{u-0}^{1,2}=\left(\frac{p_{e}}{\rho_{e}}\right)^{1.2}
$$

where $c_{s}$ is the sound speed of the medium (or the propagation velocity of the particles in the lattice). Taking the differential on both sides of (2.21) and combining equations (2.20), (2.21), and (2.22), yields a linear wave equation for small perturbations in density,

$$
\nabla^{2} \rho_{e}=\frac{1}{c_{s}^{2}} \frac{\partial^{2} \rho_{e}}{\partial t^{2}}
$$

Equation (2.23) can also be expressed for small perturbations in pressures or flow velocity. In more general terms, the HPP lattice gas automaton can thus model conditions described by the following linear scalar wave equation, 


$$
\nabla^{2} \Phi=\frac{1}{c_{s}^{2}} \frac{\partial^{2} \Phi}{\partial t^{2}}
$$

The HPP-LGA algorithm was used for this thesis to model electromagnetic phenomena. The $\Phi$ term in the linear scalar wave equation of (2.22) can be equated to the electric field $E_{z}$, or magnetic field $H_{;}$, amplitudes when considering two-dimensional, transverse-magnetic (TM:), or transverse-electric ( $\mathrm{TE}_{\mathbf{z}}$ ) wave propagation, respectively, with the application of appropriate boundary conditions and sources [2].

\subsubsection{Macroscopic Observables}

Quantities such as the density and flow of a fluid are determined by the macroscopic behaviour of the lattice gas. To recover the linear wave equation, an "ensemble average" [21,22] must be performed over a local group of cells. A sampling region $R$, is therefore selected and defined at a specified location within the lattice. The total number of particles is determined within the sampling region, and then averaged over the number of cells in the window for every time step. An example of a square sampling window is shown in Figure 2.6 .

The microscopic density $\rho_{\text {cell }}$ and velocity $\boldsymbol{u}_{\text {cell }}$ of a particular cell $j$ is defined as [23],

$$
\begin{gathered}
\rho_{c e l l}\left(x_{j} y_{j}\right)=\sum_{i=1}^{4} b_{i}\left(x_{j} y_{j}\right) \\
u_{c e l d}\left(x_{j} y_{j}\right)=\sum_{i=1}^{+} b_{i}\left(x_{j} y_{j}\right) c^{i}
\end{gathered}
$$

where $x_{j}, y_{j}$ denote the coordinates of cell $j$, and $b_{i}\left(x_{j}, y_{j}\right)$ indicates the velocity state $i(i=1$, $2,3,4$, for west, north, east, and south) of cell $j$. The presence of a particle is defined by, $b_{i}$ $=1$, and the absence of a particle by $b_{i}=0$. 


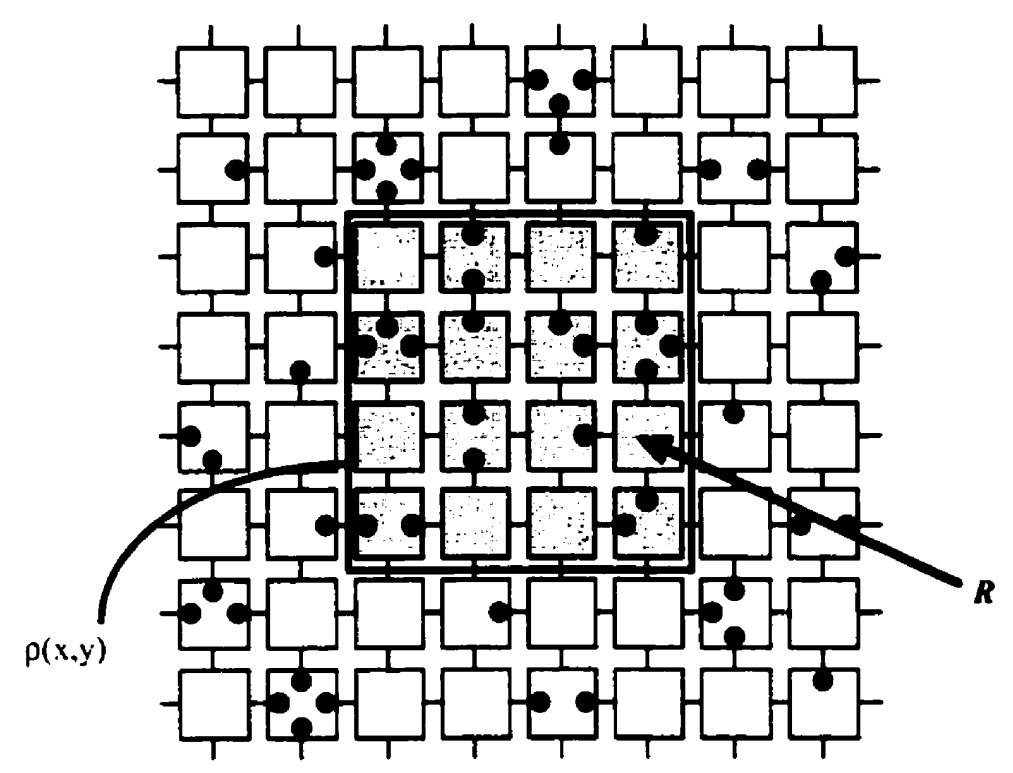

Figure 2.6: Example of a square sampling window over region $R$.

The macroscopic (or spatial) density and velocity at a particular location within the lattice is determined by the averaging of the microscopic states over a group of cells. This consists of averaging the values of a group of $\rho_{\text {cell }}$, or $\boldsymbol{u}_{\text {cell }}$, respectively,

$$
\begin{aligned}
& \rho(x, y)=\frac{1}{N_{T}} \sum_{R}\left(\sum_{i=1}^{+} b_{i}\left(x_{R} y_{R}\right)\right) \\
& \boldsymbol{u}(x, y)=\frac{2}{N_{T}} \sum_{R}\left(\sum_{i=1}^{+} b_{i}\left(x_{R} y_{R}\right) c^{i}\right)
\end{aligned}
$$

where the window $R$ is the neighbourhood centered around $\left(x_{2} y\right),\left(x_{R}, y_{R}\right)$ is the coordinate of a particular cell within $R$, and $N_{T}$ is the total number of bit variables within $R$.

Wave propagation by the HPP-LGA model is achieved by introducing a wave (density perturbation) at a particular location within the defined lattice. The HPP rules govern the interaction of the particles at the cellular level, and allow the propagation of the wave throughout the system. When modelling a two-dimensional TM, wave using the HPP. 
LGA algorithm, the microscopic electric field at a particular cell $j$ will be defined as the density of particles in the cell [2], i.e. $E_{\text {s.cell }}=\rho_{\text {cell }}$,

$$
E_{z, c e l l}\left(x_{j}, y_{j}\right)=\sum_{i=1}^{+} b_{i}\left(x_{j}, y_{j}\right)
$$

The macroscopic electric field $E_{z}$, is analogous to the macroscopic density of a particular region of cells. The values of $E_{i c e l l}$ are averaged within the sampling window over $R$ as follows,

$$
E_{z}(x, y)=\frac{1}{N_{T}} \sum_{R}\left(\sum_{i=1}^{+} b_{i}\left(x_{R} v_{R}\right)\right)
$$

The microscopic flow velocity $u=\left(u_{x}, u_{y}\right)$ of a particular cell is analogous to the microscopic magnetic field $\boldsymbol{H}=\left(-H_{y}, H_{x}\right)$ (discussed in section 2.4.2). The macroscopic $H$ fields are thus described by the macroscopic flow velocity, and can be simplified as,

$$
\begin{aligned}
& H_{x}=\frac{2}{N_{T}} \sum_{R}\left(b_{2}-b_{4}\right) \\
& H_{y}=-\frac{2}{N_{T}} \sum_{R}\left(b_{1}-b_{3}\right)
\end{aligned}
$$

In the case of a two-dimensional $\mathrm{TE}_{z}$ wave, the magnetic field $H_{z}$ would be equated to the macroscopic density, and the electric field components, $E_{x}$, and $E_{y}$, to the macroscopic velocity. 


\subsection{Similarities Between Fluid Flow and Electromagnetic Propagation}

Similarities between fluid flow and wave propagation suggest that lattice gas automata may be used for the analysis of electromagnetic phenomena. The scalar analysis of acoustic waves is analogous to a similar electromagnetic system, and will be illustrated for the reflection of a sound wave and an EM wave at a plane boundary between two uniform regions [24].

A time-harmonic plane wave traveling in the $x-y$ plane, indicated in Figure 2.7, can be expressed in the following form,

$$
\Phi\left(x_{y} y\right)=A e^{j\left(k_{\mathrm{r}} x \cdot k_{y} y\right)}
$$

where $A$ is the field amplitude, $k_{x}=|\mathbf{k}| \cos (\phi), k_{y}=|\mathbf{k}| \sin (\phi)$ are the $x$ and $y$ components of the propagation vector $\mathbf{k}$ from the origin to a point on the wave front, and the magnitude of $k$ is $|k|=2 \pi / \lambda$ or $\omega / c$.

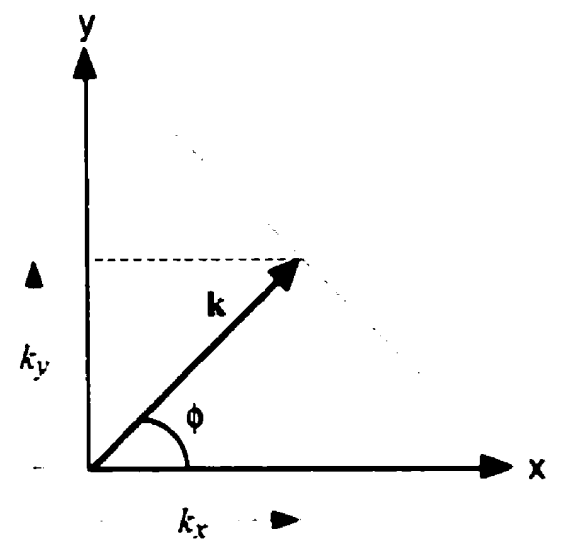

Figure 2.7: Wave propagation vector [8]. 


\subsubsection{Analysis of Acoustic Waves at a Plane Boundary}

The reflection of an acoustic wave at a plane boundary is shown in Figure 2.8. The incident, reflected and transmitted pressure field components can be expressed as follows,

$$
\begin{aligned}
& p_{i}=A e^{j\left(k_{1_{r}} x+k_{1_{y}} y\right)} \\
& p_{r}=B e^{j\left(-k_{t_{r}} x-k_{1_{y}} y\right)} \\
& p_{t}=C e^{j\left(k_{z_{r}} x-k_{y_{r}} y\right)}
\end{aligned}
$$

where $A, B$, and $C$ are the wave amplitudes.

The flow velocity fields in region 1 and 2 can be determined from the defined pressure fields by defining the wave impedances, $Z_{1}$ and $Z_{2}\left(Z=\rho c_{s}\right.$, where $c_{s}$ is the velocity of propagation of the medium), in the two regions,

$$
\begin{array}{ll}
u_{i_{\mathrm{r}}}=\frac{p_{i}}{Z_{1}} \cos \left(\phi_{1}\right) & u_{i_{\mathrm{y}}}=\frac{p_{i}}{Z_{1}} \sin \left(\phi_{1}\right) \\
u_{r_{\mathrm{r}}}=-\frac{p_{r}}{Z_{1}} \cos \left(\phi_{1}\right) & u_{r_{\mathrm{y}}}=\frac{p_{r}}{Z_{1}} \sin \left(\phi_{1}\right) \\
u_{t_{\mathrm{r}}}=\frac{p_{t}}{Z_{2}} \cos \left(\phi_{2}\right) & u_{t_{\mathrm{y}}}=\frac{p_{t}}{Z_{2}} \sin \left(\phi_{2}\right)
\end{array}
$$



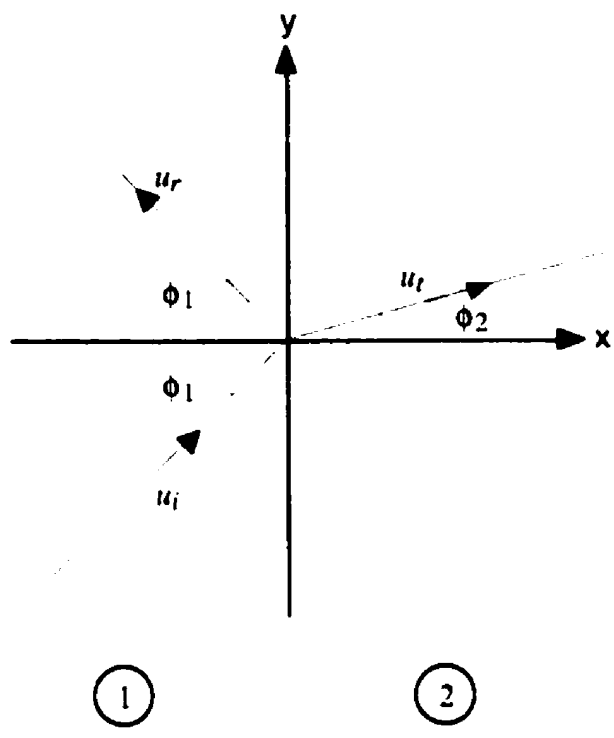

Figure 2.8: Reflection of an acoustic wave at a plane boundary [8].

The conditions at the boundary are defined as,

$$
\begin{aligned}
p_{i}-p_{r} & =p_{t} \\
u_{i_{\mathrm{r}}}-u_{r_{\mathrm{r}}} & =u_{t_{\mathrm{r}}}
\end{aligned}
$$

The regions must have the same pressure amplitudes and normal velocity if there is to be no penetration of one medium into the other at the boundary $x=0$. The $y$-dependent variables on both sides should be the same $\left(k_{i y}=k_{3 y}\right)$. Using equations (2.32) to (2.38), the boundary conditions can be expressed as,

$$
A-B=C \text { and }\left(\frac{A-B}{Z_{1}}\right) \cos \left(\phi_{1}\right)=\left(\frac{C}{Z_{2}}\right) \cos \left(\phi_{2}\right)
$$

The reflection and transmission coefficients, $\Gamma$ and $\tau$, can be defined from (2.39),

$$
\begin{aligned}
& \Gamma=\frac{B}{A}=\frac{Z_{2} \cos \left(\phi_{1}\right)-Z_{1} \cos \left(\phi_{2}\right)}{Z_{2} \cos \left(\phi_{1}\right)+Z_{1} \cos \left(\phi_{2}\right)} \\
& \tau=\frac{C}{A}=\frac{2 Z_{2} \cos \left(\phi_{1}\right)}{Z_{2} \cos \left(\phi_{1}\right)+Z_{1} \cos \left(\phi_{2}\right)}
\end{aligned}
$$




\subsubsection{Analysis of Electromagnetic Waves at a Plane Boundary}

The reflection of an EM wave at a plane boundary is shown in Figure 2.9 for the TM. case. The corresponding equations for the electric field in the $z$-direction are,

$$
\begin{aligned}
& E_{i_{z}}=A^{\prime} e^{j\left(k_{1_{x}} x-k_{1} y\right)} \\
& E_{r_{z}}=B^{\prime} e^{j\left(-k_{1_{\mathrm{r}}} x-k_{1_{y}} y\right)} \\
& E_{t_{z}}=C^{\prime} e^{j\left(k_{z_{r}} x \cdot k_{y_{y}} y\right)}
\end{aligned}
$$

where $A^{\prime}, B^{\prime}$, and $C^{\prime}$ are the amplitudes of the incident, reflected, and transmitted electric field components.
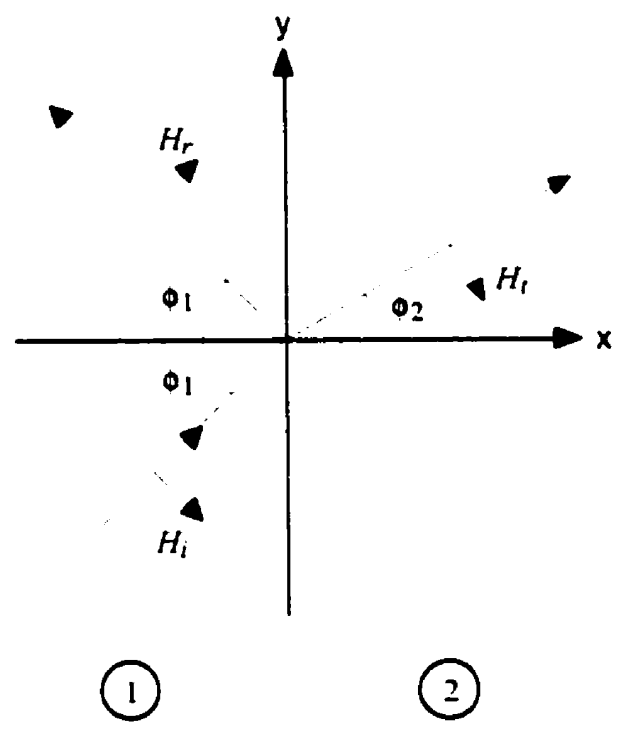

Figure 2.9: Reflection of a TM: wave at a plane boundary [8]. 
The magnetic field in the $x$ and $y$-directions can be obtained using the wave impedance $Z=(\mu / \epsilon)^{1 / 2}$, where the incident, reflected, and transmitted components shown in Figure 2.9, are

$$
\begin{array}{ll}
H_{i_{\mathrm{v}}}=-\left(\frac{E_{i_{z}}}{Z_{1}}\right) \cos \left(\phi_{1}\right) & H_{i_{\mathrm{r}}}=\left(\frac{E_{i_{z}}}{Z_{1}}\right) \sin \left(\phi_{1}\right) \\
H_{r_{v}}=\left(\frac{E_{r_{z}}}{Z_{1}}\right) \cos \left(\phi_{1}\right) & H_{r_{\mathrm{r}}}=\left(\frac{E_{r_{z}}}{Z_{1}}\right) \sin \left(\phi_{1}\right) \\
H_{t_{\mathrm{v}}}=-\left(\frac{E_{t_{z}}}{Z_{2}}\right) \cos \left(\phi_{2}\right) & H_{t_{\mathrm{r}}}=\left(\frac{E_{t_{z}}}{Z_{2}}\right) \sin \left(\phi_{2}\right)
\end{array}
$$

and $Z_{1}$ and $Z_{2}$ are the wave impedances in regions 1 and 2 , respectively. Thus, from the analysis detailed by equations (2.32) to (2.37) for acoustics waves and (2.42) to (2.47) for EM waves, the electric field $E_{i}$, is analogous to the pressure field, and the magnetic field components, $\boldsymbol{H}=\left(-H_{y}, H_{x}\right)$, to the flow velocity, $\boldsymbol{u}=\left(u_{x}, u_{y}\right)[2,8]$.

The boundary conditions at the interface of regions 1 and 2 require that the tangential electric (and magnetic) components be continuous across the boundary. The $E_{z}$ (and $H_{y}$ ) components must be the same in both regions. Thus, equivalent to equation (2.39), the boundary conditions are,

$$
A^{\prime}-B^{\prime}=C^{\prime} \quad \text { and } \quad\left(\frac{A^{\prime}-B^{\prime}}{Z_{1}}\right) \cos \left(\phi_{1}\right)=\left(\frac{C^{\prime}}{Z_{2}}\right) \cos \left(\phi_{2}\right)
$$

Using (2.48), the reflection and transmission coefficients become, 


$$
\begin{aligned}
& \Gamma=\frac{B^{\prime}}{A^{\prime}}=\frac{Z_{2} \cos \left(\phi_{1}\right)-Z_{1} \cos \left(\phi_{2}\right)}{Z_{2} \cos \left(\phi_{1}\right)+Z_{1} \cos \left(\phi_{2}\right)} \\
& \tau=\frac{C^{\prime}}{A^{\prime}}=\frac{2 Z_{2} \cos \left(\phi_{1}\right)}{Z_{2} \cos \left(\phi_{1}\right)+Z_{1} \cos \left(\phi_{2}\right)}
\end{aligned}
$$

which are identical to (2.40) and (2.41).

For the $\mathrm{TE}_{\text {: }}$ case, $E_{\mathrm{z}}$ is zero, with the following $E$ - and $H$-field incident, reflected, and transmitted components, shown in Figure 2.10.
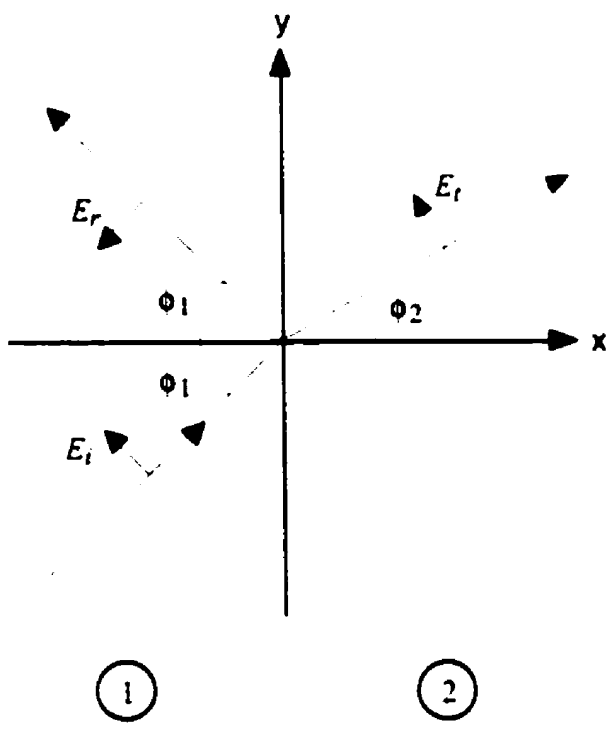

(2)

Figure 2.10: Reflection of an TE: wave at a plane boundary [8]. 


$$
\begin{gathered}
E_{i_{y}}=E_{i} \cos \left(\phi_{1}\right) \quad E_{i_{\mathrm{r}}}=-E_{i} \sin \left(\phi_{1}\right) \\
E_{r_{\mathrm{v}}}=-E_{r} \cos \left(\phi_{1}\right) \quad E_{r_{\mathrm{r}}}=-E_{r} \sin \left(\phi_{1}\right) \\
E_{t_{\mathrm{y}}}=E_{t} \cos \left(\phi_{2}\right) \quad E_{t_{\mathrm{r}}}=-E_{t} \sin \left(\phi_{2}\right) \\
H_{i_{z}}=\frac{E_{i}}{Z_{1}} \\
H_{r_{:}}=\frac{E_{i}}{Z_{1}} \\
H_{t_{z}}=\frac{E_{t}}{Z_{2}}
\end{gathered}
$$

Thus, for the TE: case, the magnetic field $\mathrm{H}_{\mathrm{i}}$ is analogous to the pressure field, and the electric field components, $\boldsymbol{E}=\left(E_{y},-E_{\jmath}\right)$, to the flow velocity, $\boldsymbol{u}=(u, u)$. Boundary conditions, and the reflection and transmission coefficients result in expressions similar to equations $(2.48)$ to $(2.50)$. 


\section{Chapter 3}

\section{ELEMENTARY LATTICE GAS EXPERIMENTS}

A fortran program was written to implement the HPP-LGA algorithm. Discussion of the following factors:

- Source excitation

- Boundary conditions

- $\quad$ Propagation speed and viscosity

- Spatial and ensemble averaging

- Effect of frequency transformation

are considered in this chapter using the lattice parameters outlined below. Figure 3.1 depicts the general properties of the lattice for the modelling of TM: field problems where $\rho \equiv E_{z}$. For the examples given in the following pages, the lattice size was kept constant to illustrate differences resulting from a specific simulation parameter.

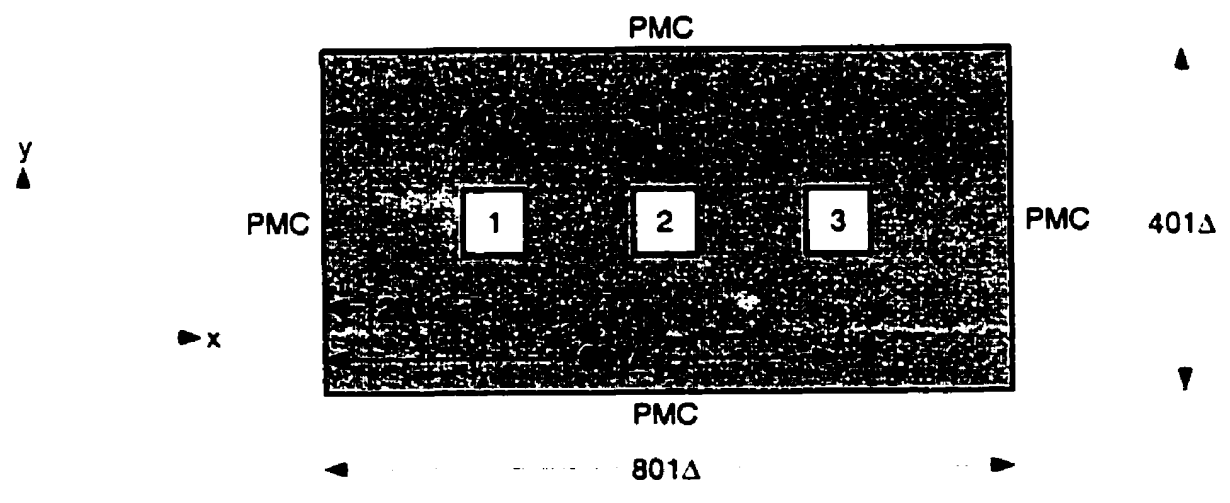

Figure 3.1: Lattice configuration. 
A rectangular lattice having a mesh size of $N_{x} \Delta x \mathrm{X} N_{y} \Delta y$ is implemented in each case, where $N_{\mathrm{r}}=801$ and $N_{y}=401$ are the total number of cells along the $x$ and $y$-directions of the lattice, and $\Delta x=\Delta y=\Delta$ is one lattice unit. A background distribution of particles was initialized with approximately $50 \%$ of the total velocity states in the lattice occupied by particles. A Gaussian pulse plane wave having a pulse width of $\sigma=40 \Delta$, propagating in the $x$-direction, is launched in the mesh with an amplitude $20 \%$ above the background particle density. Three square sampling windows with dimensions of $120 \Delta \times 120 \Delta$ were placed in the mesh, centered at $(201 \Delta, 201 \Delta),(401 \Delta, 201 \Delta)$, and $(601 \Delta, 201 \Delta)$.

\subsection{Source Excitation}

A plane wave is excited at a particular location in the lattice by superimposing a specified excitation on the background distribution of particles. In each case studied, the wave was propagated along the $x$-axis. A Gaussian pulse is generated by superimposing particles in the lattice according to the following equation for the source perturbation $\rho_{e}$,

$$
\rho_{e}=p_{m} e^{-\left(\frac{x_{n}-x_{0}}{0}\right)^{2}}
$$

where $x_{n}$ represents a particular column of cells in the $x$-direction of the lattice, and $\sigma$ represents the standard deviation of the pulse. The variable $p_{m}$ denotes the amplitude of the perturbation. The pulse is centered about $x_{o}$, and thus has its maximum, $p_{m}$, in column $x_{o}$ of the lattice. The pulse will split into two opposite traveling pulses having a maximum amplitude of $p_{m} / 2$ [2,21]. This property will be further addressed in section 3.2. Gaussian waveforms were applied to the bulk of the experiments in this thesis.

The amplitude of the source is typically selected to employ a maximum perturbation of $10 \%$ to $20 \%$ above the background distribution of particles [2]. When the amplitude of the perturbation has a magnitude greater than 0.2 , a distortion occurs in the pulse perturbation. The time-domain trace for a Gaussian pulsed plane wave is shown in Figure 
3.2 for $p_{m}=0.2,0.4,0.6$, and 0.8 . The distortion is evident by the increasingly triangular appearance of the Gaussian waveform for larger amplitude of $p_{m}$.

The wave amplitude is a combination of background density of particles in the lattice and the density of the source perturbation. By expressing the complete particle distribution of the lattice using the uniform background density $\rho_{o}$, and the small perturbation density $\rho_{e}$, the total macroscopic density $\rho$ is,

$$
\rho=\rho_{o}-\rho_{e}, \quad 0<\rho<1
$$

A background particle density of $\rho_{o}=0.5(50 \%)$ was implemented in the majority of the experiments in this thesis. Dissipative effects, with respect to the propagation velocity in the mesh and the decay of the pulse perturbation, are less significant for values of $\rho_{\mathrm{o}}$ about 0.5 . These effects are discussed in section 3.3

A random number generator (RAND) was used to fill the HPP-LGA lattice, by randomly allocating particles to the four velocity states in each cell. The program written to execute the HPP-LGA algorithm adopted an initial seed value to energize the random number generator. If the random number is less than $\rho$, a particle is placed in the corresponding cell state. Identical initial seeds call the same group of random numbers to fill the lattice. Because it is possible to duplicate simulation results by initiating the generator with the identical original seed value, the fill process is only pseudo-random.

It should be noted that in all cases discussed in this chapter and other sections of this thesis, the background distribution of particles $\rho_{o}$, has been subtracted from the averaged particle ensemble at each time step. Thus, only the small perturbation injected into the lattice is shown in each time-domain plot. A wave amplitude of zero does not imply that the no particles exist in the sampling region. 
(a)

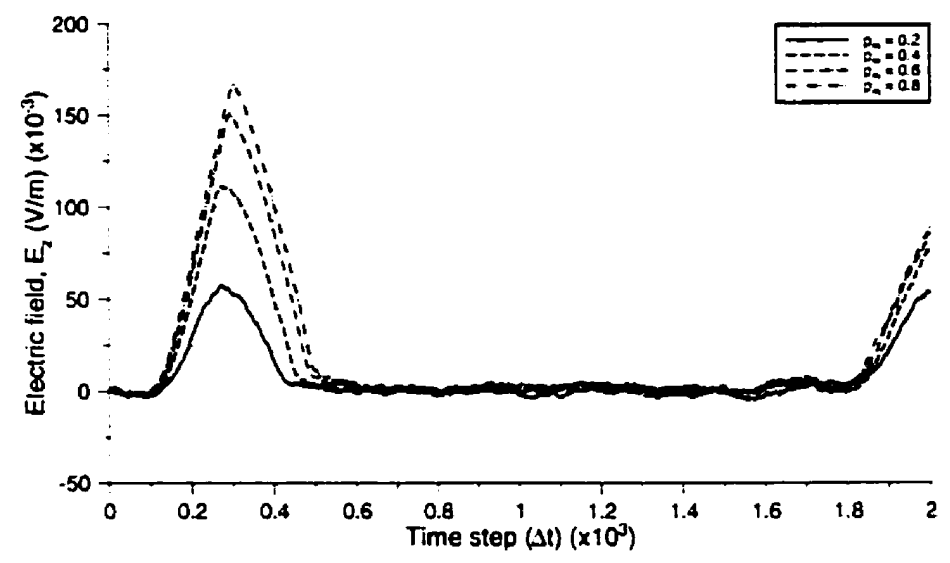

(b)

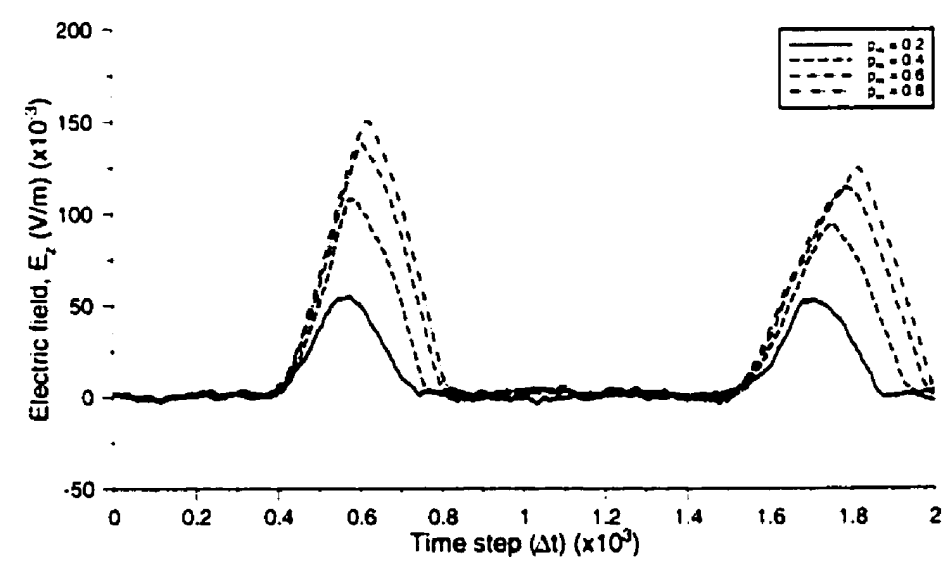

(c)

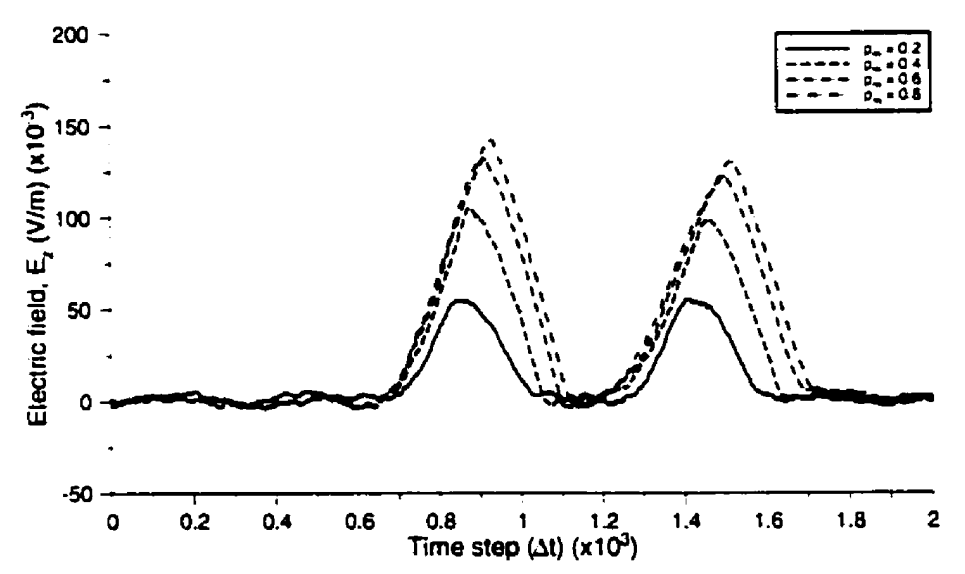

Figure 3.2: Gaussian pulsed plane wave excitation for $\rho_{m}=0.2,0.4,0.6$ and 0.8 ; (a) window 1 , (b) window 2 , and (c) window 3 . 


\subsection{Boundary Conditions}

Two types of boundary conditions are considered in this thesis. They include:
1) Perfect magnetic conducting boundary
2) Absorbing boundary

Simple plane wave propagation is simulated to illustrate the effect of each boundary. The electric field $E_{z}$ (for TM waves) is determined by the macroscopic averaging of the particles located in the cells of each window, as defined by (2.26).

\subsubsection{Perfect Magnetic Conducting Boundary}

In the $\mathrm{TM}_{\mathrm{z}}$ case, a perfect magnetic conducting (PMC) boundary is a soft boundary condition that enforces a zero microscopic tangential magnetic field at a boundary [10]. An open circuit termination with a reflection coefficient of $\Gamma=+1$, is implemented by reflecting particles that enter a cell along the PMC boundary. Particles are thus bounced back into the lattice with the opposite velocity. Figure 3.3 illustrates particles at a PMC boundary located along the top row of the illustrated lattice portion. Notice that the particles are moving in the opposite direction after each internal collision.
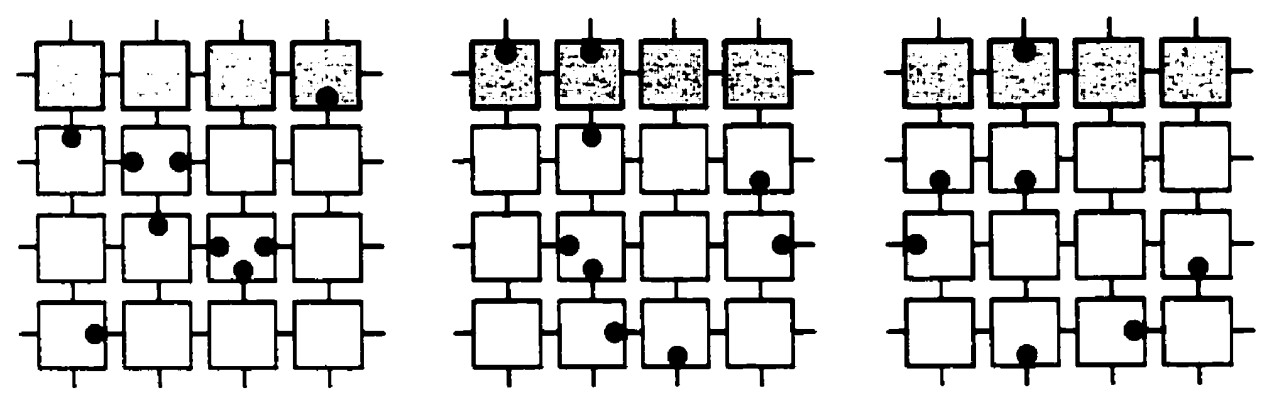

$n \Delta t$

$(n+1) \Delta t$

$(n+2) \Delta t$

Figure 3.3: Perfect magnetic conducting boundary condition [8]. 
Referring to Figure 2.8 and (2.31) to (2.33), an $x$-directed acoustic wave $\left(u_{y}=0\right)$ normal to a soft boundary undergoes a reflection equivalent to $u_{i x}=-u_{r x}$. From (2.34), $u_{x}=$ 0 for flow normal to the boundary. Using the analogy between flow and the magnetic field expressed in section 2.4 for $\mathrm{TM}_{z}$ fields, $-H_{i y}=H_{n y},\left(E_{i z}=E_{r}\right)$ at a PMC boundary.

A hard boundary condition for $\mathrm{TM}_{z}$ analysis models a perfect electric conducting (PEC) boundary, or a short circuit where $\Gamma=-1$. A dual grid system is implemented, where one lattice carries positive particles and the other negative particles $[2,10]$. The particles on each respective lattice do not interact except along the PEC boundaries. PMC boundaries do not require a dual lattice approach, since particles do not undergo a change in sign.

As an example, a Gaussian pulsed plane wave is excited at $x_{o}=201 \Delta x$, with all four walls of the lattice subject to PMC boundaries. The resulting time domain waveforms are given in Figures 3.4 (a), (b), and (c) for the calculated electric field at windows 1, 2, and 3, respectively. The pulses shown in each window do not have the same magnitude, an effect of the splitting of the Gaussian wave. The east and west PMC boundaries cause the $x$ directed wave to oscillate back and forth within the mesh.

Figure 3.4 (a) for window 1, which was centered at the exact location of the source at $x=201 \Delta x$. illustrates the original pulse at time $t=0$. The second smaller pulse is represented by the original left-directed wave after being bounced-back at the west boundary and reintroduced into the lattice in the easterly direction. Window 2 in Figure 3.4 (b) shows four pulses, the first and third resulting from the original right-directed wave, and the second and fourth which are products of the original left-directed wave.

Referring to window 3, in Figure 3.4 (c), the first pulse represents the initial right directed wave, and the third the initial left-directed wave as they reach window 3 with a magnitude $p_{m} / 2$. After originally traveling in the left-direction, the left-directed wave strikes the west boundary where it is bounced back into the mesh, in the opposite (easterly) direction. The original right-directed wave is detected by window 3 prior to striking the eastern boundary of the lattice. It is then bounced back into the lattice traveling in the westerly direction where it is re-united with the other waveform at window 3 , producing the larger pulse shown in Figure 3.4, at $t=1150 \Delta t$. 
(a)

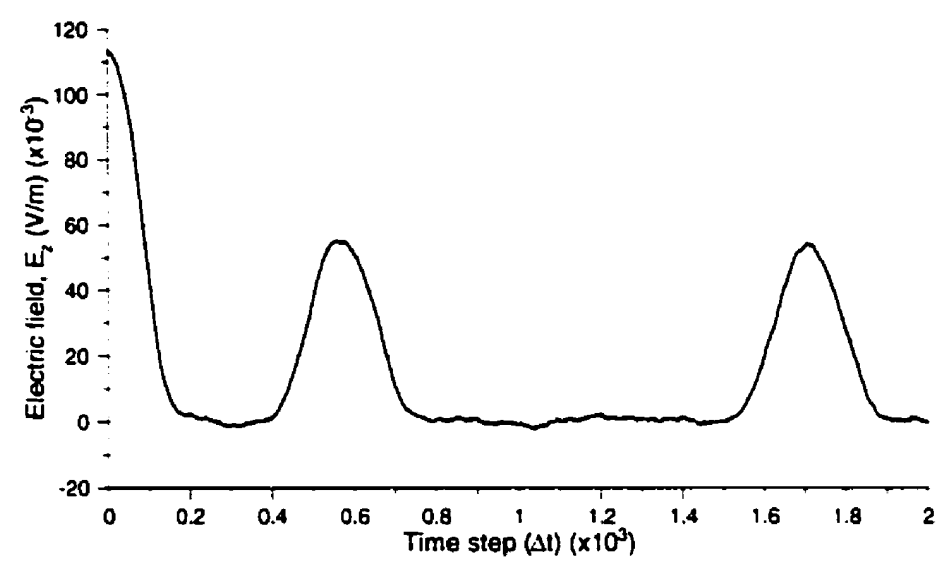

(b)
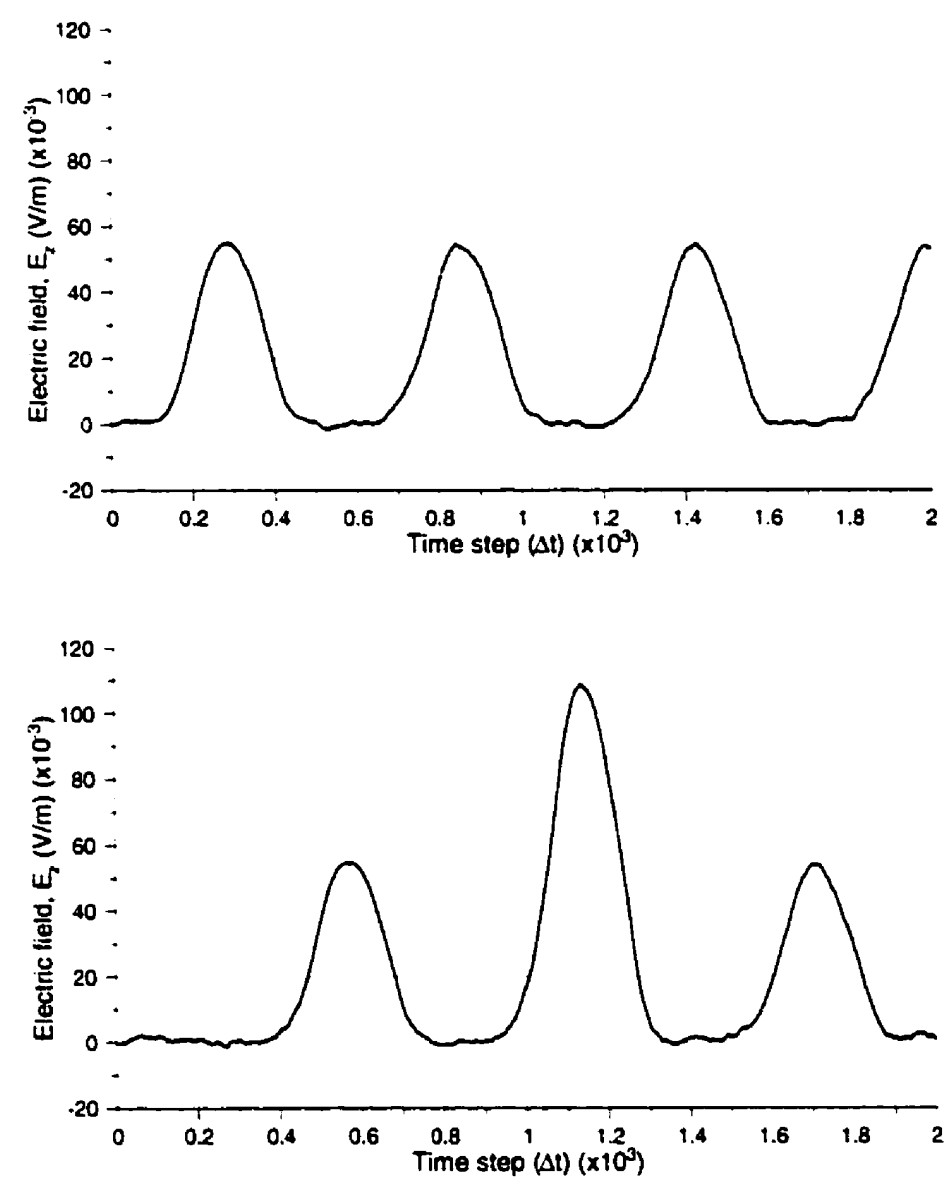

Figure 3.4: Time-domain waveforms for four walled PMC mesh with $x_{0}=2014$; (a) window 1 ; (b) window 2; (c) window 3. 
It is possible to propagate a Gaussian pulsed plane wave in only the forward direction. An alternate method of excitation allows the plane wave to evolve in the positive $x$ direction by strategically placing a PMC boundary at the location of the source excitation. Using this approach, a pulse was excited in the lattice at $x_{o}=1 \Delta x$, the first column of cells along the west wall of the lattice, also designated as a PMC boundary. By placing the center of the Gaussian pulse at the west PMC boundary, only those cells to the right of the boundary are affected by the pulse. As such, the waveform evolves in one direction only, thereby eliminating the existence of a second waveform in the lattice. This case is shown in Figures 3.5 (a), (b), and (c), for windows 1,2 , and 3, respectively. Note that the waveform magnitude is constant in all three sampling windows.

A consequence of this method is that only one half of the pulse is excited at the boundary, producing a pulse with an amplitude equivalent to $p_{m} / 2$. To obtain the desired amplitude, a value twice that of $p_{m}$ must be designated to the pulse. However, this solution is subject to the effects discussed in section 3.1 , concerning signal distortions when $p_{m}$ is not small. 
(a)

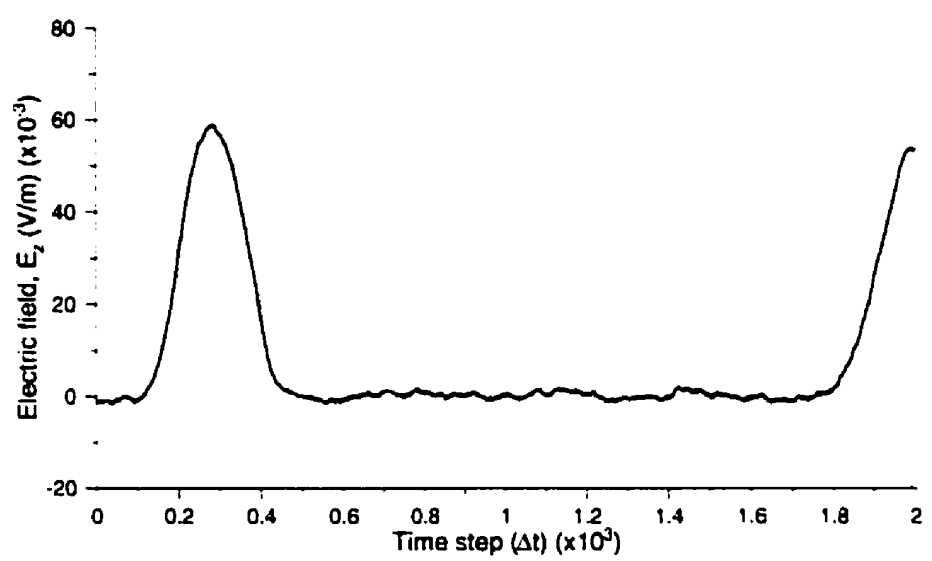

(b)
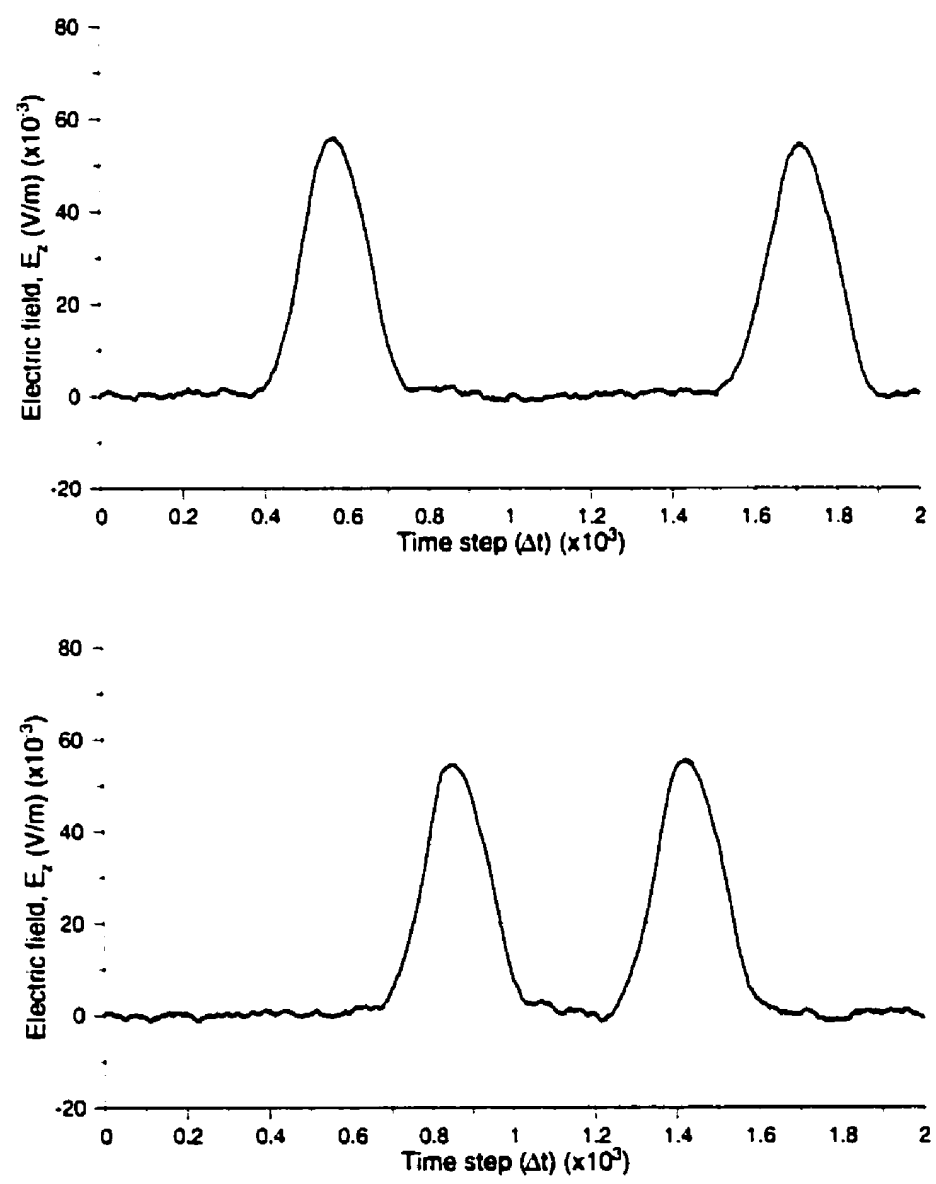

Figure 3.5: Time-domain waveforms for PMC boundaries at north, south, east, and west walls, $x_{0}=1 \Delta x$; (a) window 1 ; (b) window 2; (c) window 3 . 


\subsubsection{Absorbing Boundary Condition}

An absorbing boundary is implemented for the simulation of problems requiring an infinite space. A matched load is adequately simulated by implementing an absorbing boundary condition $(\mathrm{ABC})$ at the required location in the lattice.

A brief study of possible simulation procedures for the efficient implementation of an $A B C$ is provided in [8]. The approach selected for the implementation of an $A B C$ involves only a single column of cells. The incoming particles are absorbed by the cells along the boundary, and are subsequently re-introduced, using the random number generator to re-establish a background distribution of $\rho_{o}$. The small perturbation is not re-introduced and, ideally, is absorbed at the boundary. The single column ABC was shown by [8] to be effective in absorbing normally incident waves, having a reflection coefficient, $\Gamma$, of $-15 \mathrm{~dB}$.

Propagation of a one-dimensional plane wave was initiated in a mesh having PMC boundaries on the north, south, and west walls. A single column ABC is initialized on the east wall. In order to ensure that only one wave is propagating through the lattice, a Gaussian pulse is excited on the PMC boundary at $x_{o}=1 \Delta x$. Figures 3.6 (a), (b), and (c) show the time-domain waveforms for windows 1,2 , and 3 , respectively. Note that only a single pulse is prominent in each window. Upon close examination however, it would appear that a reflection due to the absorbing boundary (as opposed to a true physical reflection) is traveling back through the lattice. This is an indication that the single column $A B C$ is not a perfect absorbing boundary. It is observed at approximately $1999 \Delta t$ for window $1,1714 \Delta t$ for window 2 , and $1425 \Delta t$ for window 3.

To assure that the phenomena is indeed precipitated by the poor accuracy of the absorbing boundary, identical simulations were performed for mesh sizes of $801 \Delta \times 601 \Delta$ and $801 \Delta \times 801 \Delta$, to determine whether the reflection was consistent for larger mesh sizes or simply a result of edge diffraction by the smaller lattice. The absorbing boundary reflection was observed in both experiments, with the resulting waveforms provided in Figure 3.7 for the $801 \Delta \mathrm{X} 801 \Delta$ lattice. 
(a)

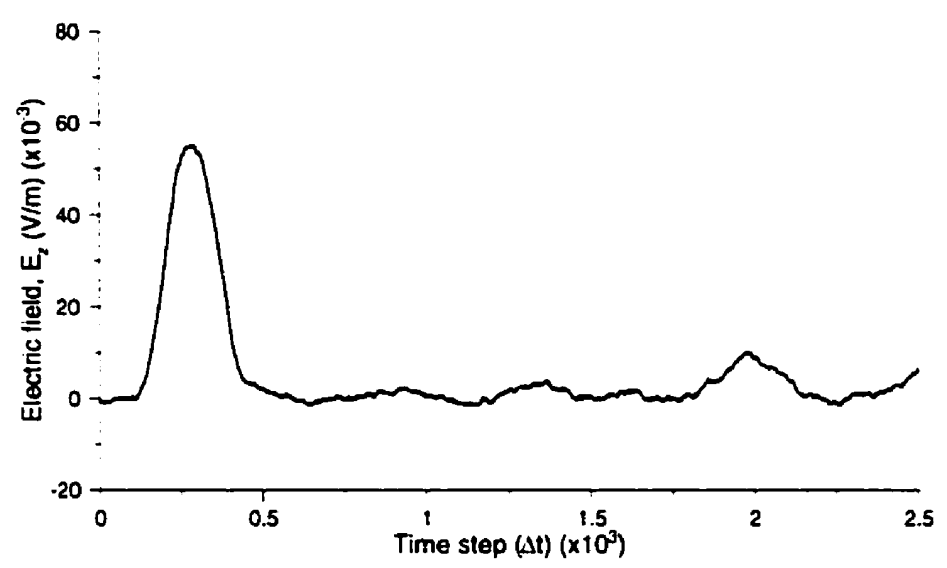

(b)

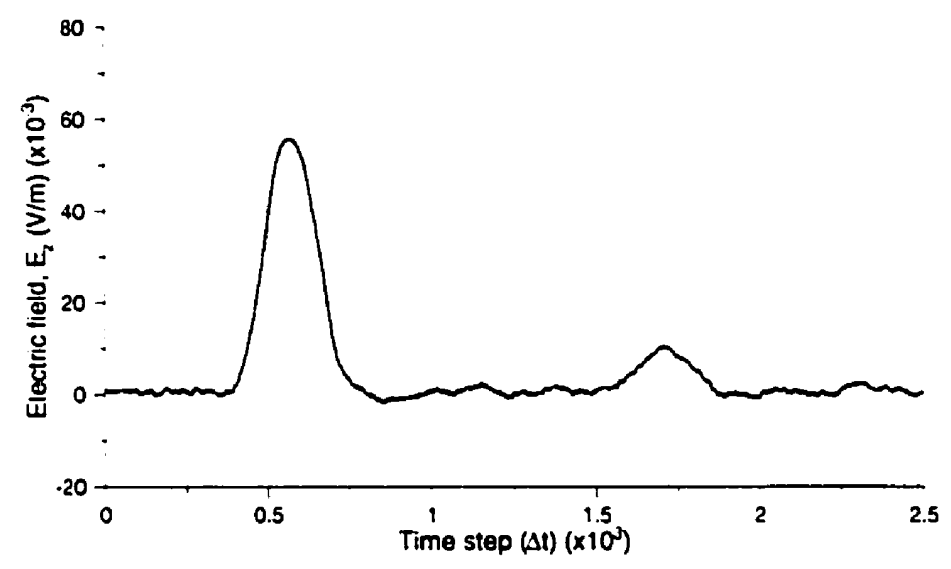

(c)

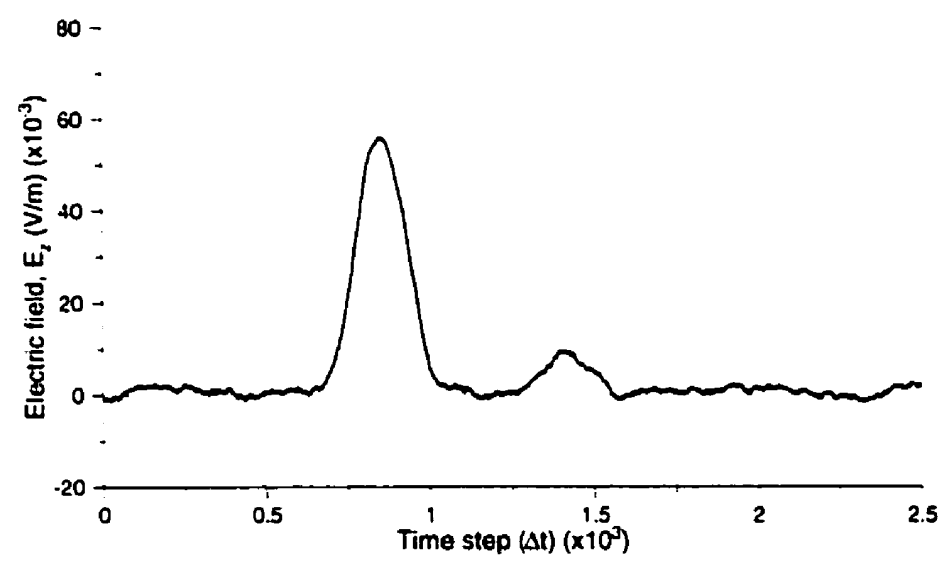

Figure 3.6: Time-domain waveforms for north, south, and west PMC walls, $A B C$

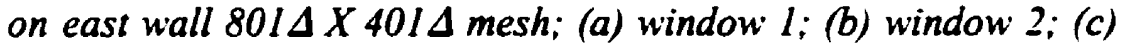
window 3. 
(a)

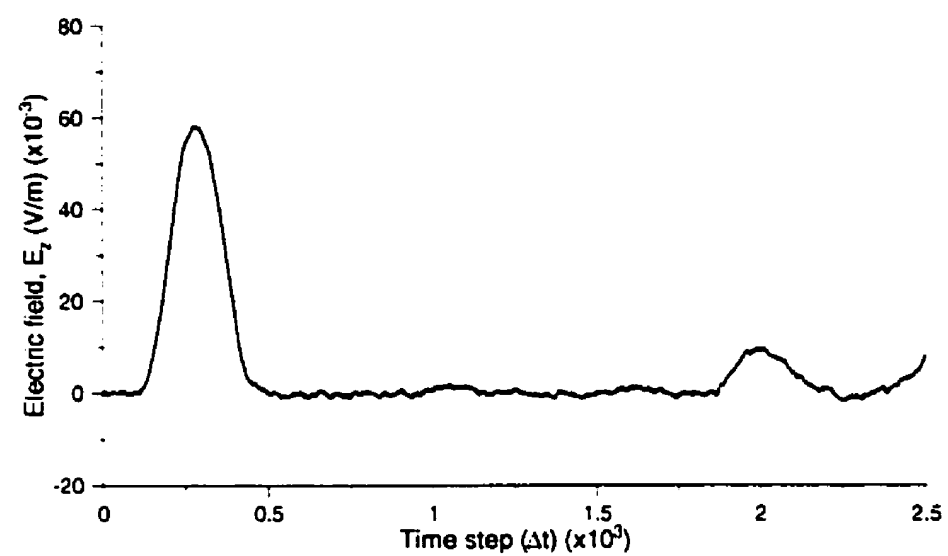

(b)

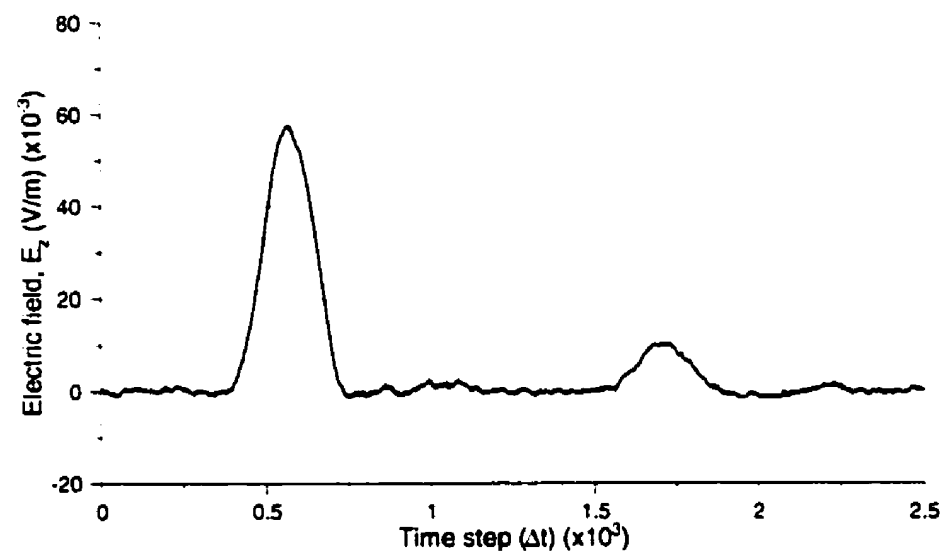

(c)

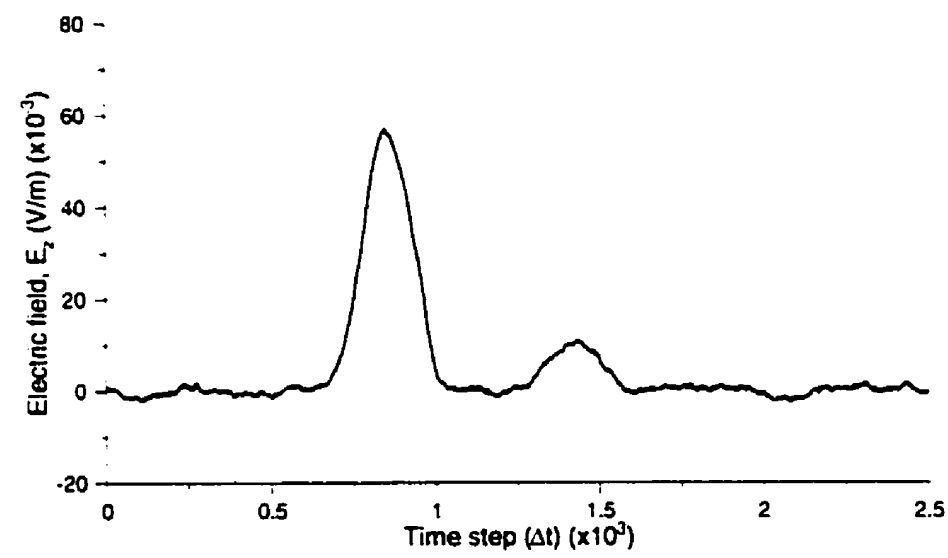

Figure 3.7: Time-domain waveforms for north, south, and west PMC walls, $A B C$ on east wall, $801 \Delta X 801 \Delta$ mesh; (a) window 1; (b) window 2; (c) window 3. 
The ratio of the $\mathrm{ABC}$ reflection to the incident pulse was determined by considering the waveform decay through the lattice. This exponential decay,

$$
A\left(x_{2}\right)=A\left(x_{1}\right) e^{-\alpha\left(x_{2}-x_{1}\right)}
$$

where $A$ represents the pulse amplitude at locations $x_{1}$ and $x_{2}$ in the lattice, and $\alpha$ represents the pulse decay, is a result of the LGA viscosity and is addressed in section 3.3.2. Using the perturbation decay $\alpha=0.4597 \times 10^{-4}$ for the decay of the incident pulse between the third window and the $A B C$ wall (from section 3.3.2), and the decay of the reflection between the $A B C$ wall and the third window, the reflection coefficient $\Gamma$ of the $A B C$ was determined as follows,

$$
\Gamma=\frac{R e^{-2 \alpha x}}{A}=-15 d B
$$

where $R=0.00947 \mathrm{~V} / \mathrm{m}$ and $A=0.05607 \mathrm{~V} / \mathrm{m}$, are the amplitudes of the $A B C$ reflection and the incident pulse at window 3, respectively, and $x=200 \Delta$, the distance of window 3 to the east $\mathrm{ABC}$ wall. The reflection coefficient has an amplitude of $-15 \mathrm{~dB}$, in agreement with [8], or $18 \%$ of the incident pulse.

It is possible to assign an $A B C$ on the west wall of the mesh, without affecting the source excitation at $x_{0}=1 \Delta x$, by imposing a time delayed boundary condition on the west wall. The west wall is assigned a PMC boundary until such time as $3 \sigma$ had elapsed. Once the pulse has traveled far enough away from the wall, the PMC boundary is then switched to an absorbing boundary. Neither the incident pulse nor the $\mathrm{ABC}$ reflection amplitudes are affected by the time-delayed boundary condition. 


\subsection{Propagation Velocity and Viscosity}

\subsubsection{Propagation velocity}

The sound speed, $c_{s}$, in the lattice can be determined by selecting specific observation points on the pulse and the recurrence of these points established by the time steps and spatial distance on the lattice between them. The number of cells divided by the number of iterations would thus provide the sound speed.

As an example. Table 3.1 lists the calculated sound speed from the data obtained by each of the three sampling windows in the simulation outlined in section 3.2.1.1, for the forward travelling Gaussian in the four-walled PMC mesh. For the waveform of each sampling window, an observation point was placed at the peak of the incident pulse. The time between the recurrence of this point was established, as well as the number of cells travelled by the pulse. Note that $\Delta l=\Delta$, one lattice unit, and that each time step has a value of $\Delta t=1.0$.

The sound speed in the HPP-LGA lattice has been determined to be $[2,10]$,

$$
c_{s}=\frac{1}{\sqrt{2}}
$$

or 0.7071 , independent of $\rho_{o}$, for a lattice spacing of $\Delta x=\Delta y=\Delta l$, and a time step of $\Delta t$.

Table 3.1: Time at each observation point, their spatial distance, and sound speed in each sampling window.

\begin{tabular}{|c|c|c|c|c|}
\hline Window & of cells $(\Delta l)$ & Time, $t_{l}(\Delta t)$ & Time, $t,(\Delta t)$ & $c_{s}$ \\
\hline 1 & 1200 & 289 & 1991 & 0.7051 \\
\hline 2 & 800 & 573 & 1707 & 0.7055 \\
\hline 3 & 400 & 854 & 1409 & 0.7117 \\
\hline
\end{tabular}




\subsubsection{Viscosity}

The HPP-LGA technique does not exhibit the numerical dispersion errors inherent with difference equations, but it does exhibit dissipative effects similar to viscosity in a fluid [25]. The viscosity influences the waveform appearance over time with the damping of the magnitude of the pulse as it propagates through the lattice. It must therefore be considered as a source of error when observing data. A method of increasing the number of bits in the representation of the particles ("multi-bit" LGA) was shown to reduce the dissipative effects of LGA [25]. Specifically, a generalization of LGA has been proposed using low-precision integer variables as opposed to single-bit variables [26]. This new algorithm was further developed in [27] and was considered as an integer lattice gas automata (ILGA) of the standard TLM approach. Recent study presented in [28] demonstrates minimal dissipation by the combined ILGA and TLM algorithm.

The speed of propagation of the lattice is expected to remain constant at $c_{s}=1 / \sqrt{2}$ $\Delta l / \Delta t$ for homogeneous systems, regardless of the background density, as stated in the previous section. However, numerical simulations show that the velocity in the mesh is influenced by the particle density of the lattice. Eight cases were simulated, each with a different background density, ranging from $\rho_{o}=0.25$ to 0.60 . A Gaussian pulse was excited at $x_{0}=1 \Delta x$ in a lattice having PMC boundaries on all four walls. Table 3.2 lists the speed determined for each value of $\rho_{o}$, and is shown plotted in Figure 3.8. The theoretical value of the lattice sound speed is superimposed on the measurement data for reference. The computed lattice speed for background particle densities of 0.25 to 0.35 and 0.55 to 0.60 show the greatest variation from theory. The measured sound speed between 0.40 and 0.50 are most similar to the expected theoretical value. The data in Table 3.2 details a similar trend, with regard to the lattice sound speed, to findings presented in [7]. 
Table 3.2: Propagation velocities for $\rho_{0}=0.25$ to 0.60 .

\begin{tabular}{|c|c|}
\hline Background density, $\rho_{o}$ & $\begin{array}{c}\text { Propagation velocity, } c_{s} \\
(\Delta / / \Delta t)\end{array}$ \\
\hline 0.25 & 0.7229 \\
\hline 0.30 & 0.7199 \\
\hline 0.35 & 0.7117 \\
\hline 0.40 & 0.7109 \\
\hline 0.45 & 0.7063 \\
\hline 0.50 & 0.7051 \\
\hline 0.55 & 0.6936 \\
\hline 0.60 & 0.6948 \\
\hline
\end{tabular}

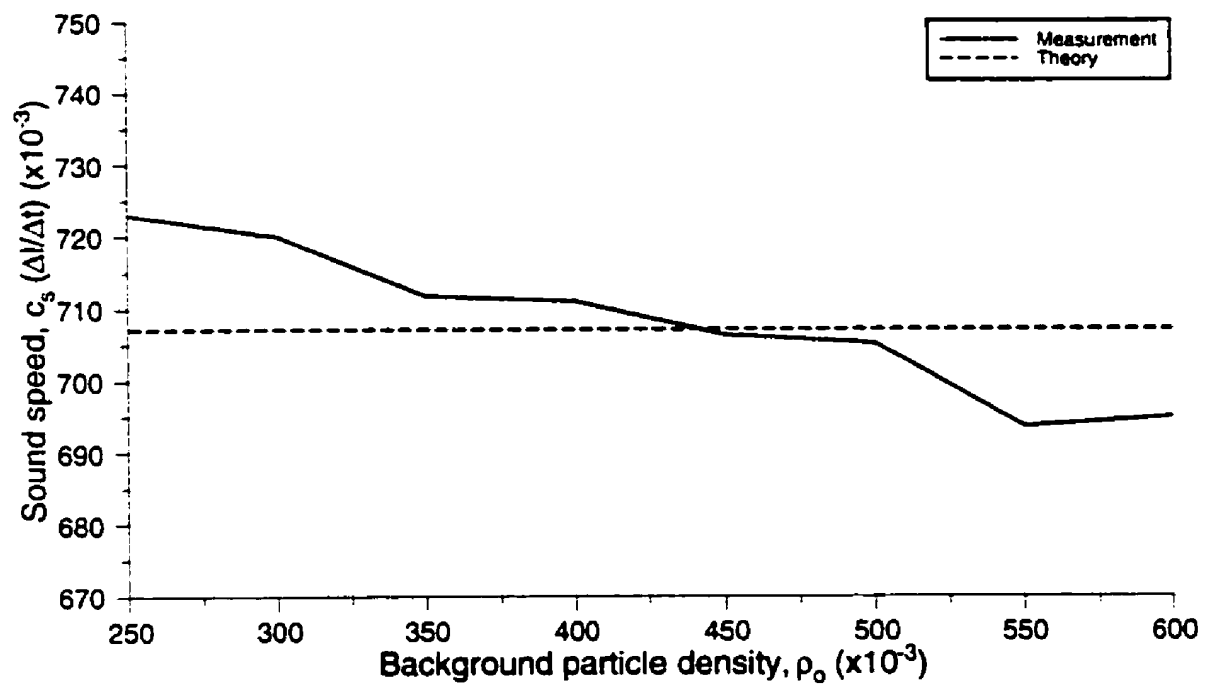

Figure 3.8: $\quad$ Lattice sound speed, $c_{s}$ vs. background distribution of particles, $\rho_{o^{*}}$. 
The decay, $\alpha$, of the perturbation was determined by computing the energy loss of the pulse observed by the second window at approximately $4000 \Delta t$ and comparing it with the incident pulse initially observed by the same window at $500 \Delta t$. An exponential decay is assumed, having the following form,

$$
A\left(x_{2}\right)=A\left(x_{1}\right) e^{-\alpha\left(x_{2}-x_{1}\right)}
$$

where $A$ represents the pulse amplitude at locations $x_{1}$ and $x_{2}$ in the lattice, and $\alpha$ represents the pulse decay. Table 3.3 lists the computed value of $\alpha$, also shown plotted in Figure 3.9, for the eight cases.

Figure 3.9 details an increased magnitude of the decay for background densities of 0.25 to 0.35 and 0.55 to 0.60 . The decay of the perturbation is least significant for background densities of 0.40 to 0.50 . This trend is similar to that observed in Figure 3.8 regarding the variation of the measured sound speed in the lattice. The decay of the perturbation pulse width is observed in the waveforms for $\rho_{o}=0.25,0.35,0.45$, and 0.55 , in Figure 3.10 . 
Table 3.3: Computed decay, $\alpha_{2}$ for $\rho_{0}=0.25$ to 0.60 .

\begin{tabular}{|c|c|c|c|}
\hline$\rho_{o}$ & Incident pulse $(\mathrm{V} / \mathrm{m})$ & Final pulse $(\mathrm{V} / \mathrm{m})$ & Decay, $\alpha\left(\times 10^{-4}\right)$ \\
\hline 0.25 & 0.05555 & 0.03846 & 1.5317 \\
\hline 0.30 & 0.05637 & 0.04515 & 0.9249 \\
\hline 0.35 & 0.05668 & 0.04821 & 0.6744 \\
\hline 0.40 & 0.05723 & 0.05103 & 0.4774 \\
\hline 0.45 & 0.05680 & 0.05149 & 0.4090 \\
\hline 0.50 & 0.05613 & 0.05027 & 0.4597 \\
\hline 0.55 & 0.05603 & 0.04723 & 0.7120 \\
\hline 0.60 & 0.05571 & 0.04409 & 0.9748 \\
\hline
\end{tabular}

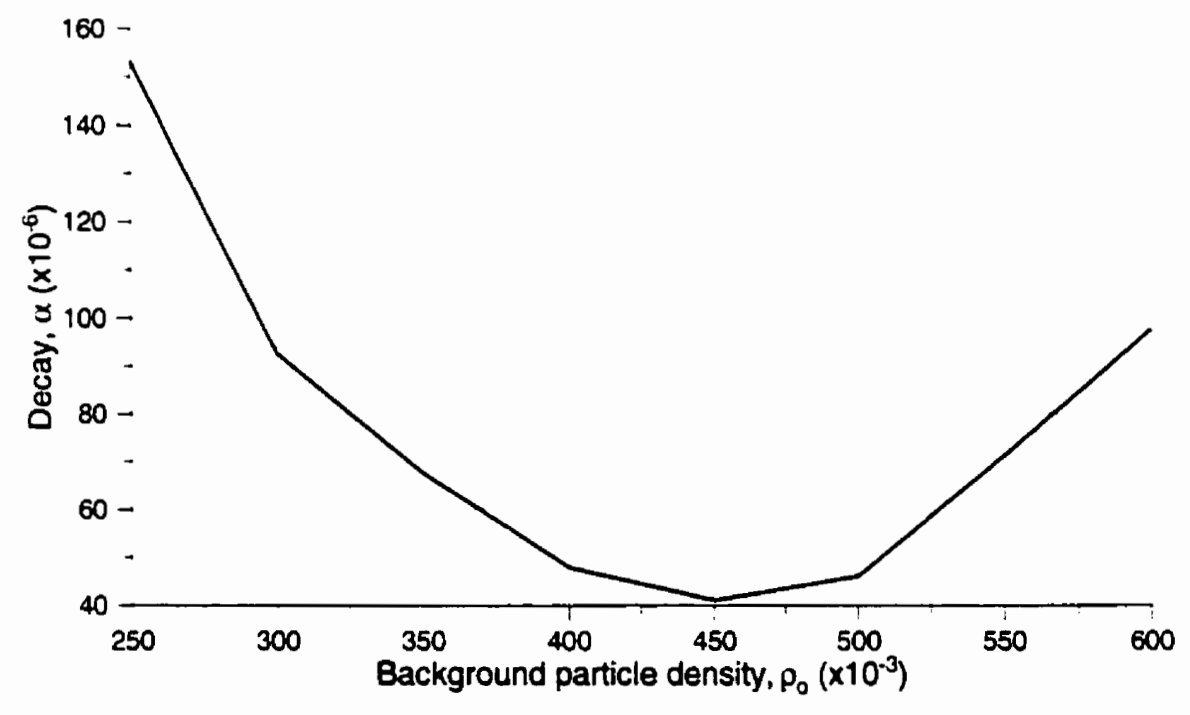

Figure 3.9: Computed decay, $\alpha$ vs. background distribution of particles, $\rho_{o^{\circ}}$. 


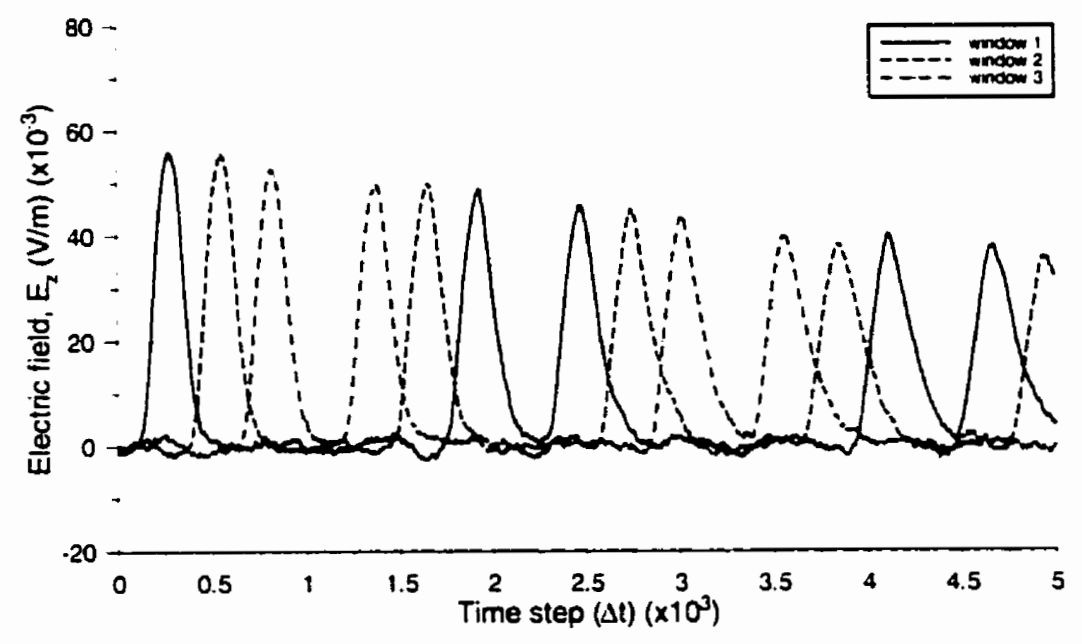

(a)

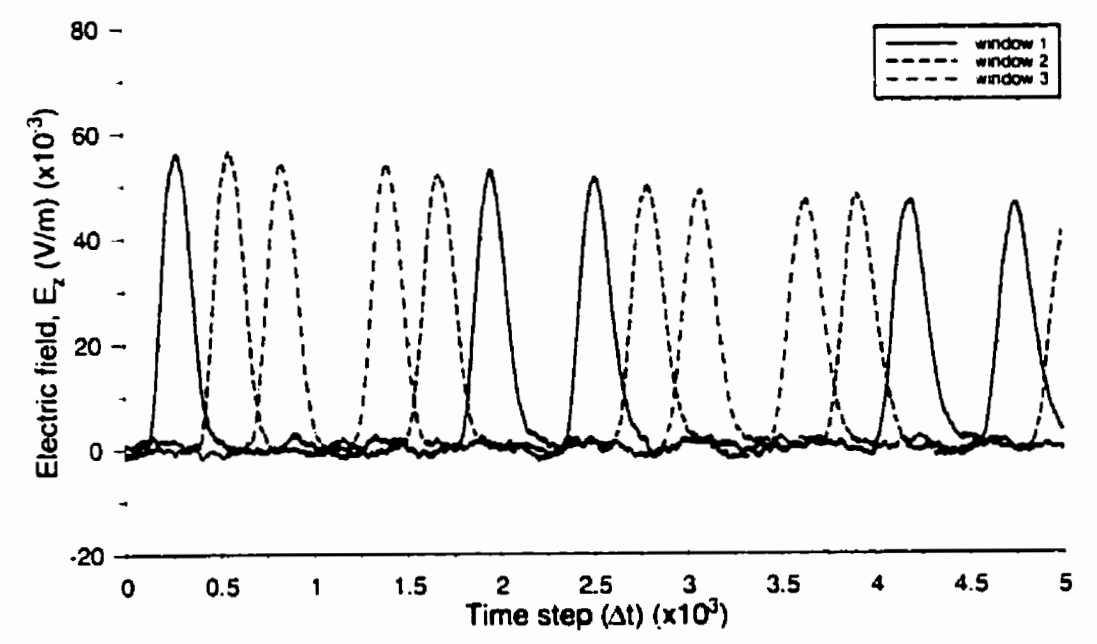

(b)

Figure 3.10: Time domain waveforms for $801 \Delta \times 401 \Delta$ mesh with four PMC walls; (a) $\rho_{o}=0.25$; (b) $\rho_{o}=0.35$; (c) $\rho_{o}=0.45$; (d) $\rho_{o}=0.55$. 


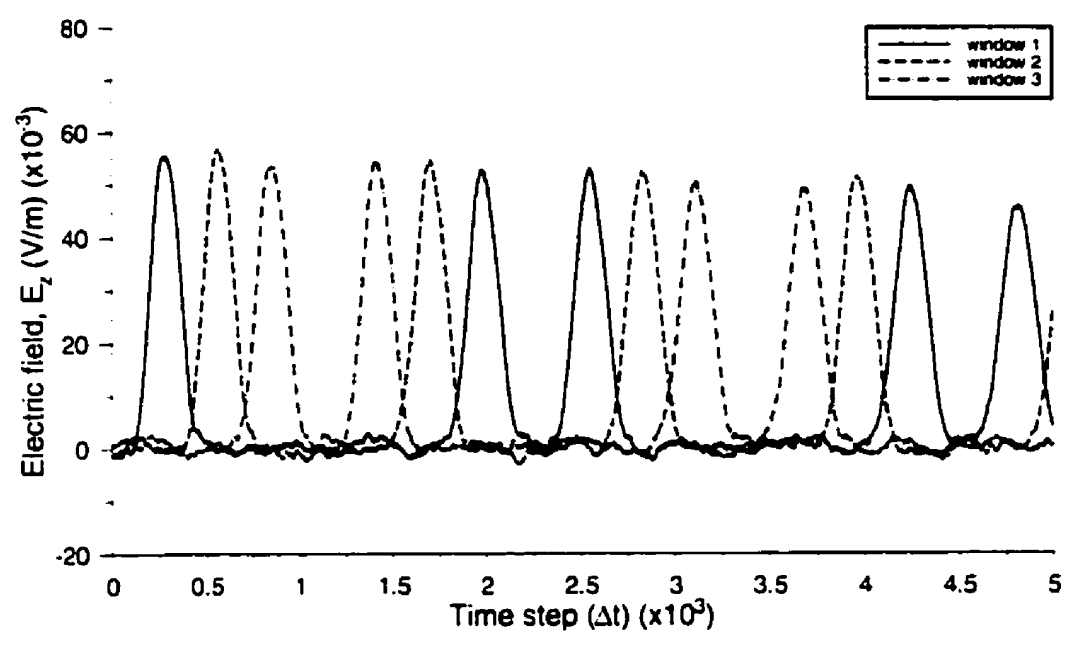

(c)

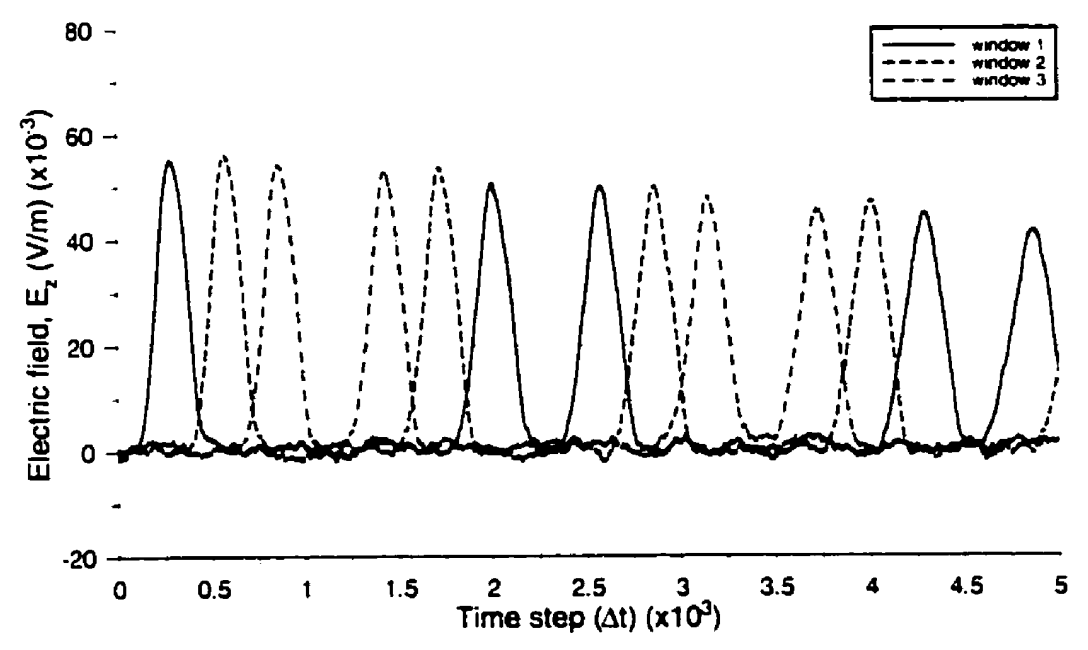

(d)

Figure 3.10 (continued) 


\subsection{Averaging}

Results shown in previous figures illustrate that noise is an inherent property of the lattice gas automaton. The noise results from the random background distribution of particles within the lattice and depends on the size of the sampling window [10]. Smoothing of the data can be achieved by averaging the data in space, as well as time [12], discussed in sections 3.4.1 and 3.4.2.

\subsubsection{Spatial Averaging}

Spatial averaging refers to the macroscopic averaging within a specified sampling region $R$. To illustrate the impact of spatial averaging on the propagating wave, three cases were simulated with square windows of varying dimensions; $80 \Delta \times 80 \Delta, 120 \Delta \times 120 \Delta$. and $160 \Delta \times 160 \Delta$. A uniform background density of $\rho_{o}=0.5$ was applied to the mesh. An increase in window size results in the broadening and damping of the pulse, shown in Figures 3.11 (a) to (c), due to the filtering of the signal by the sampling window. The broadening and damping of the pulse with regard to spatial averaging does not progress with time. It is a spatial occurrence and any further change in the waveform can be attributed to an inaccuracy due to viscosity.

A higher degree of noise is observed with a decrease in window size. The random distribution of background particles in the lattice contributes a root mean square (rms) noise level, proportional to $\sqrt{N_{T}}$ [2]. From section 2.3.2, $N_{T}$ represents the number of possible bits in the sampling window, where the sum of the actual particle bits in the window is averaged by $N_{T}$. The theoretical rms noise corresponding to a particular window becomes $\sqrt{N_{T}} / N_{T}$. Larger windows provide a larger sampling size for spatial averaging, and therefore show smoother and less coarse waveforms over the entire iteration process. 
(a)

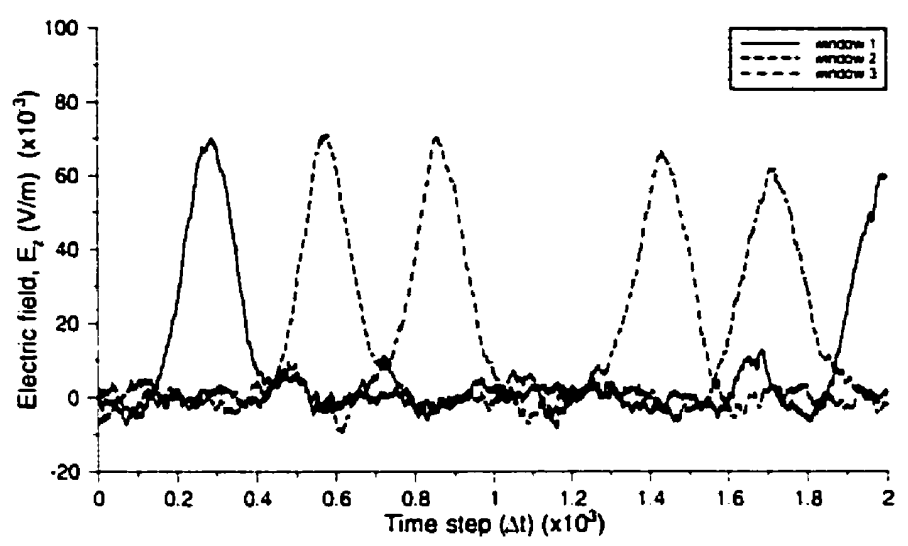

(b)

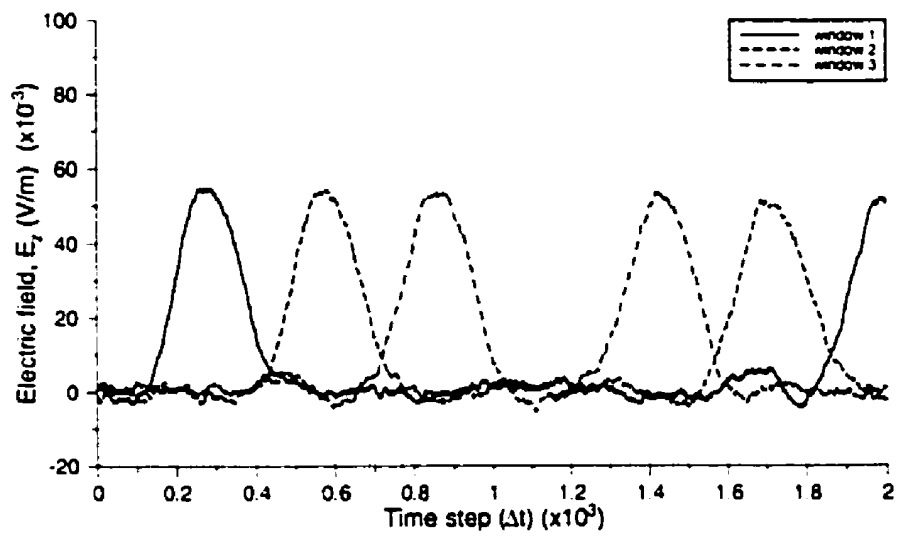

(c)

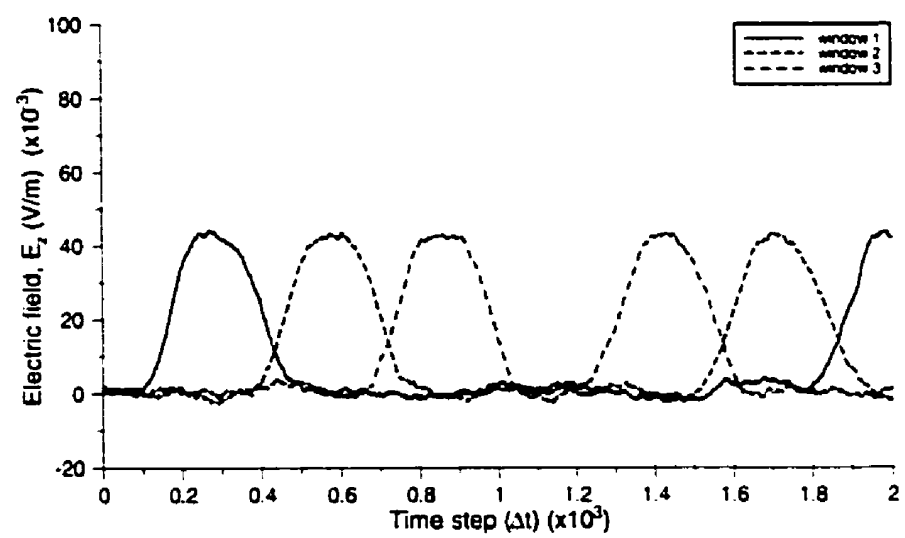

Figure 3.11: Time domain waveforms for $801 \Delta X 401 \Delta$ mesh with four PMC walls; window sizes (a); 804X 804; (b) 1204X 1204; (c) $160 \Delta X$ 1604 . 


\subsubsection{Ensemble Averaging}

The HPP-LGA algorithm is a stochastic process where the parameters of interest are macroscopic averages. In computer simulations, a pseudo-random number generator is necessary to initialize the HPP lattice. Therefore, while the algorithm involves a stochastic process, the outcome is exactly repeatable by a computer. In an attempt to create a more random experiment, experiments were executed a number of times using different seeds [7]. To eliminate correlations due to the pseudo random number generator, and to reduce the noise in the system, results are calculated using an ensemble of simulations. The data is then averaged for each iteration over the entire ensemble of simulations. In doing so, it is possible to reduce the noise by ensemble averaging.

To illustrate the influence of ensemble averaging on the noise level, a single simulation was compared with an ensemble of 5, 10, and 20 runs, each case having no source excitation. Figure 3.12 details the result for each ensemble. The noise level in each plot is less significant with an increasing number of simulations. Table 3.4 lists the ms noise data for each ensemble of simulations. Comparing the rms noise from the ensemble of 5,10 and 20 runs to the single simulation data, the noise reduction by each ensemble can be expressed as $\sqrt{N_{\text {ensemble }}}$, where $N_{\text {ensemble }}$ represents the number of runs in each simulation ensemble.

Table 3.4: Rms noise levels for single, 5, 10, and 20 ensemble simulations.

\begin{tabular}{|c|c|c|}
\hline Number of simulations & Measured ms noise & ms noise* $\sqrt{N_{\text {ensemble }}}$ \\
\hline 1 & 0.002754 & - \\
\hline 5 & 0.001026 & 0.002294 \\
\hline 10 & 0.000768 & 0.002429 \\
\hline 20 & 0.000640 & 0.002862 \\
\hline
\end{tabular}




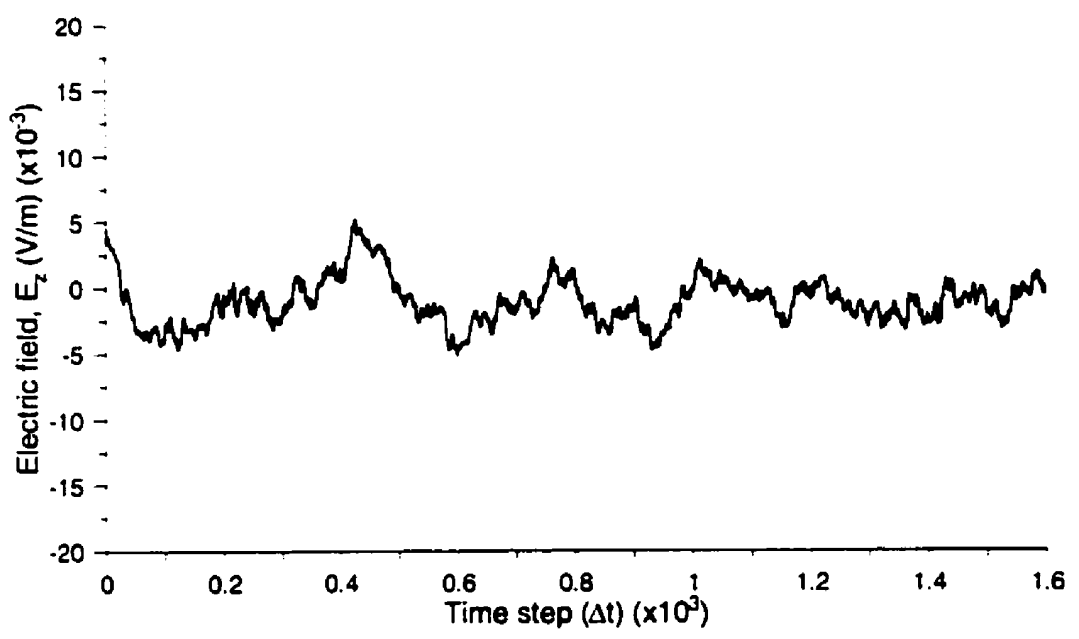

(a) 1 run

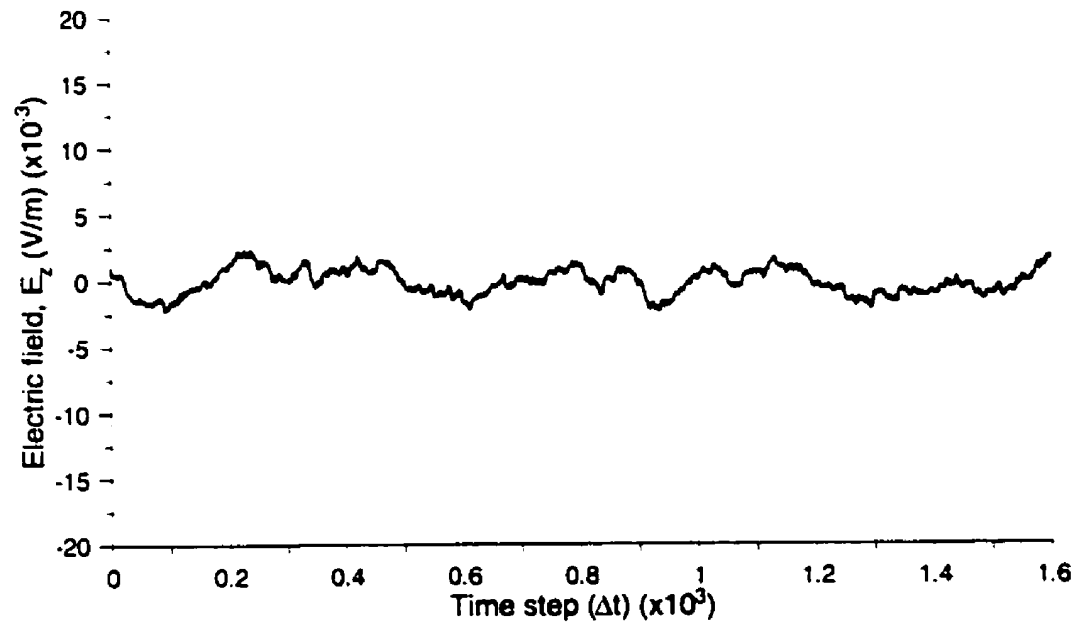

(b) 5 runs

Figure 3.12: Noise levels for 8014X 4014 mesh PMC walls and 1204X $120 \Delta$ windows; (a) I run; (b) 5 runs; (c) 10 runs; (d) 20 runs. 


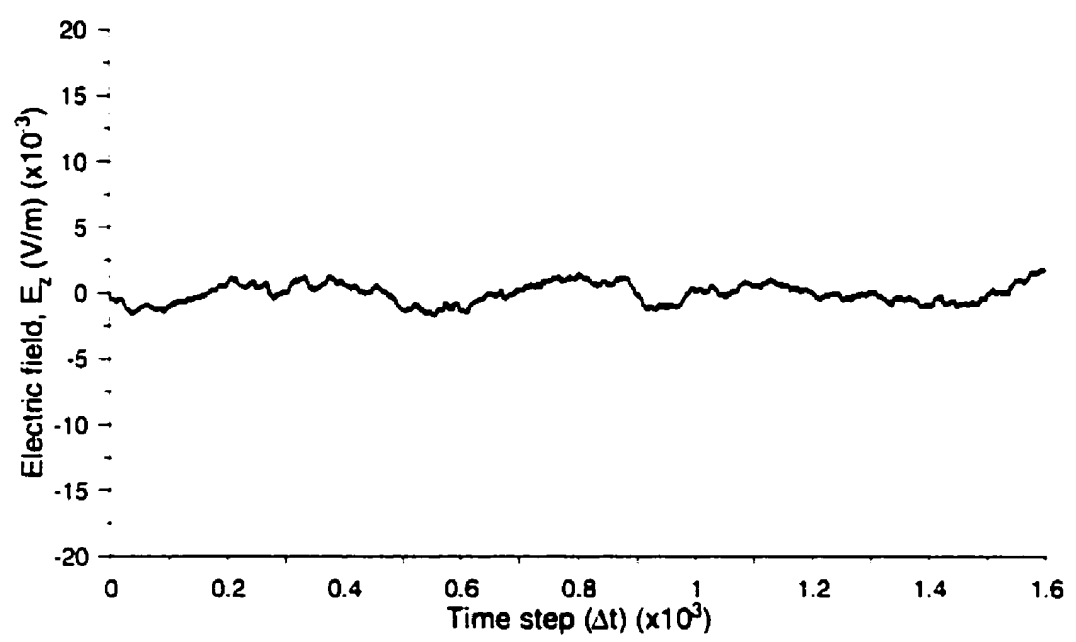

(c) 10 runs

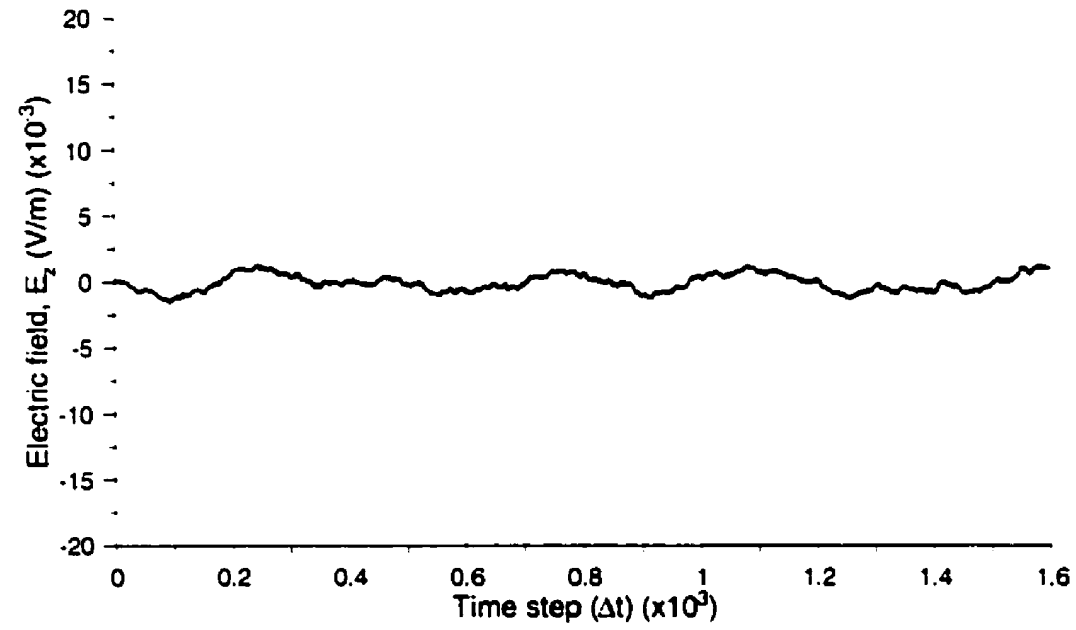

(d) 20 runs

Figure 3.12 (continued) 
Figure 3.13 shows simulations for the four walled PMC case excited at $x_{o}=1 \Delta x$, for a single simulation, as well as an ensemble of 5, 10, and 20 runs. The waveform is cleaner and less noisy with an increase in the number of simulations. A significant reduction is observed in the 20 run output with regard to noise. Thus, the 20 run ensemble displays the most desirable output. In terms of the dynamics and the smoothing of the pulse, there appears to be little change between the 10 and 20 run ensemble simulations. This is also observed in Table 3.5 regarding their respective rms noise levels. Because the simulations for this thesis were performed on a serial computer environment, and not a parallel computing machine, a 10 run ensemble is satisfactory from the standpoint of simulation practicality, as well as signal noise, and dynamics. 


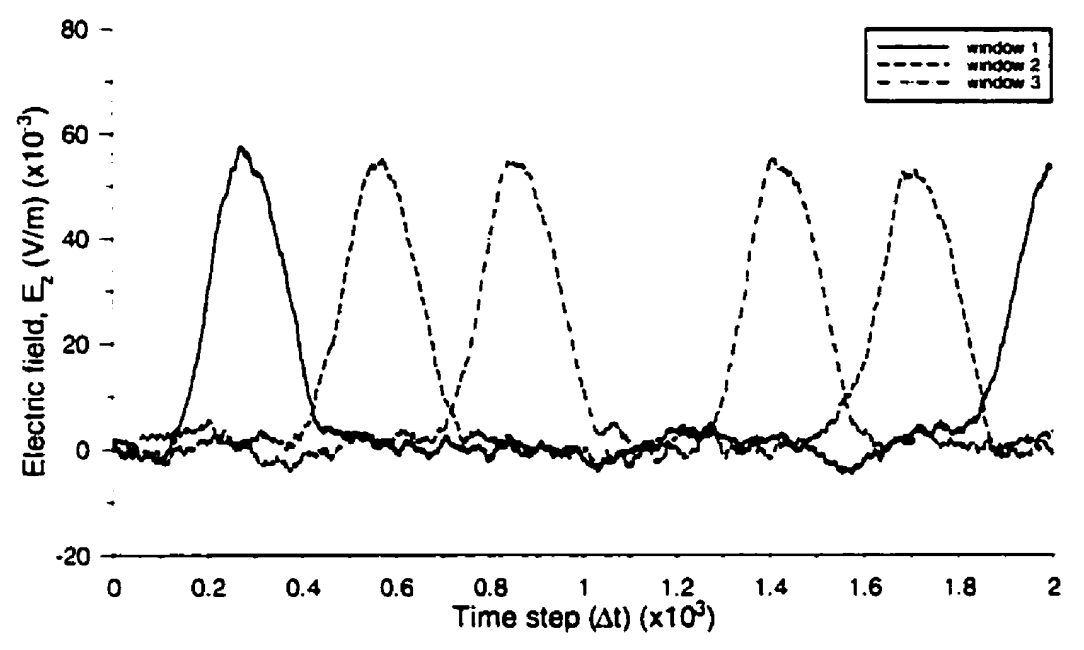

(a) 1 run

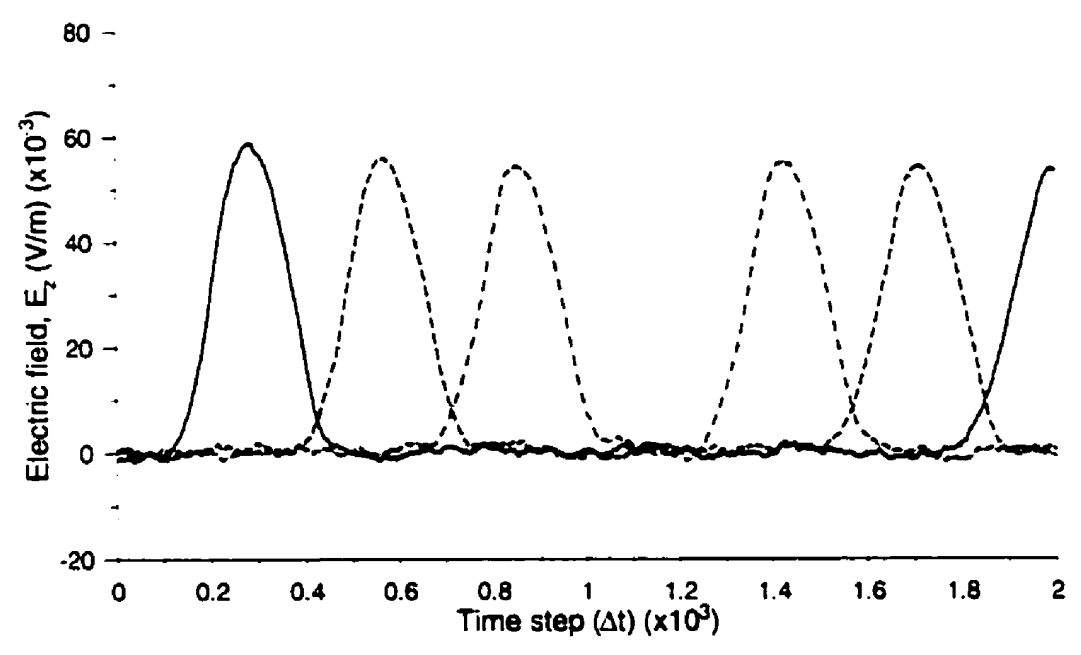

(b) 5 runs

Figure 3.13: Waveforms for (801 $\triangle X$ 401 $\Delta$ ) mesh with $P M C$ walls and (1204X 1204) windowis; (a) 1 run; (b) 5 runs; (c) 10 runs; (d) 20 runs. 


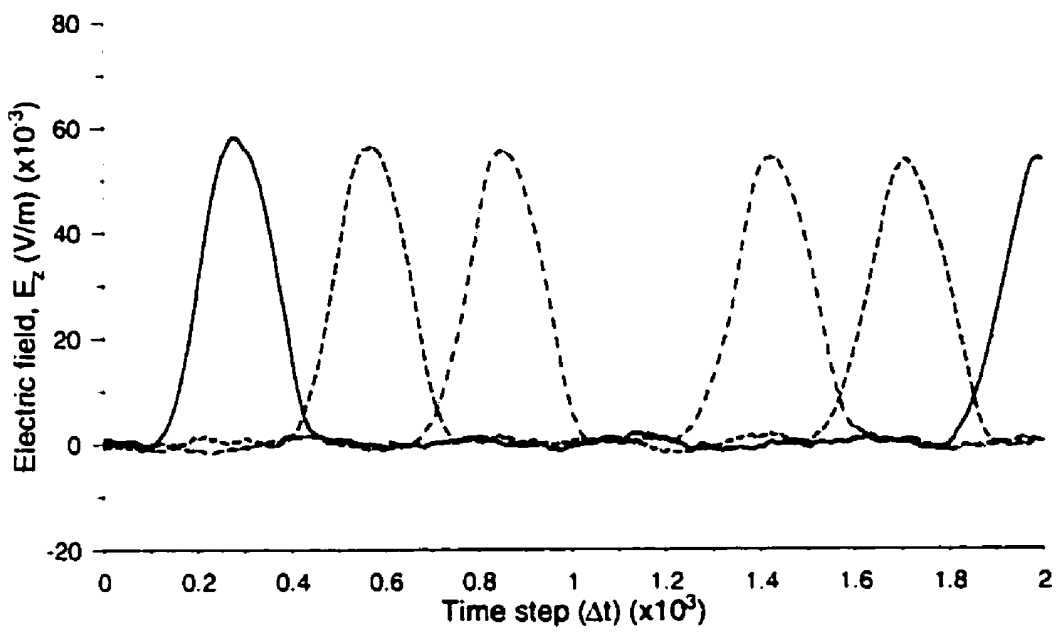

(c) 10 runs

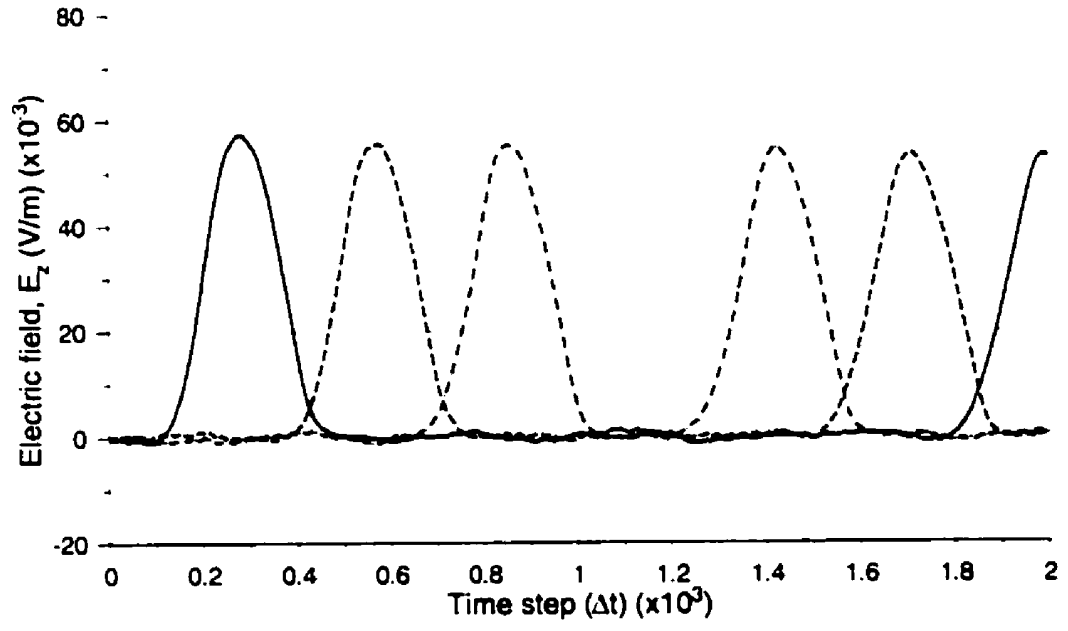

(d) 20 runs

Figure 3.13 (continued) 


\subsection{Dimensional Translation}

The frequency response of a time-domain waveform is obtained by computing the discrete Fourier transform (DFT),

$$
F(k)=\sum_{n=0}^{. V-l} f(n) e^{-j k \omega n \Delta t}
$$

where $N$ is the number of sample values, $\Delta t$ is the sampling time interval, and $\omega=$ $(2 \pi) /(N \Delta t)$. The signal length is equal to $(N-1) \Delta t$. When computing the time-domain response, each iteration in the mesh corresponds to,

$$
\Delta t=c_{s} \Delta l
$$

where $c_{s}$ is the sound speed in the mesh.

The source excitation expressed in equation (3.1) represents a Gaussian waveform in the spatial domain. The waveform can however be expressed in terms of time by scaling the pulse with respect to the speed of propagation in the lattice. Referring to the pulse width of $\sigma=40 \Delta l$, in the time domain,

$$
\sigma=40 \Delta l=40 \sqrt{2} \Delta t
$$

The incident pulse can be expressed in terms of time, along with its Fourier transform as [29],

$$
p_{m} e^{-\left(\frac{t}{\sigma}\right)^{2}}-p_{m} \sigma \sqrt{\pi} e^{-\frac{\sigma^{2} \omega}{2}}
$$

The sampling window, defined spatially for the $x$-directed waveform, can be related to the time-domain. The scaling width $a$ of the window interrelates the width of the window and the iteration time step as, 


$$
a=x_{w i n} \Delta l \equiv x_{w i n} \sqrt{2} \Delta t
$$

where $x_{\text {win }}$ is the number of cells along the $x$-directed length of the window. A similar scaling can be done for the window dimensions in the $y$ direction.

The sampling window is correctly defined by the finite gate function. The HPP-LGA lattice is two-dimensional, and thus the square sampling window is characterized by a twodimensional Fourier transform. However, the Fourier transformation of the $x$ and $y$ components consists of two explicit functions. Since the wave propagation is restricted to the $x$-direction of the HPP lattice, the transform with respect to the $y$ component of the window can be neglected. Therefore, the transform pair corresponding to the $x$ component is [30],

$$
\left\{\begin{array}{ll}
A, & |z| \leq \frac{a}{2} \\
0, & |z|>\frac{a}{2}
\end{array} \quad-\quad A a \frac{\sin (\omega a)}{\omega a}\right.
$$

where $A$ is the amplitude of the gate.

The effect of the gate function $w(t)$, on the excitation function $g(t)$, is the convolution of the two functions in the time-domain,

$$
\int_{-\infty}^{\infty} g(\tau) w(t-\tau) d \tau=g(t) * w(t)
$$

Consequently, the frequency domain response results from the product of the Fourier transform of the gate function $W(\omega)$, with the Fourier transform of the excitation $G(\omega)$,

$$
g(t) * w(t)-G(\omega) W(\omega)
$$

For a unit area gate function, the magnitude of the sinc function in the frequency 
domain at $\omega=0$ is 1 . Thus, $A=1 / a$, and the Fourier transform of the Gaussian excitation convolved with the sampling window gate function becomes,

$$
F(\omega)=\left(p_{m} \sigma \sqrt{\pi} e^{-\frac{\sigma^{2} \omega^{2}}{2}}\right) \frac{\sin (\omega a)}{\omega a}
$$

The magnitude of the Fourier transform of the incident pulse shown in Figure 3.4 (a) for a four walled PMC lattice, is given in Figure 3.14. Phase was not considered in these calculations. The spectral response illustrates the product of the Fourier transform of the Gaussian pulsed plane wave excitation with the Fourier transform of the window gate function.

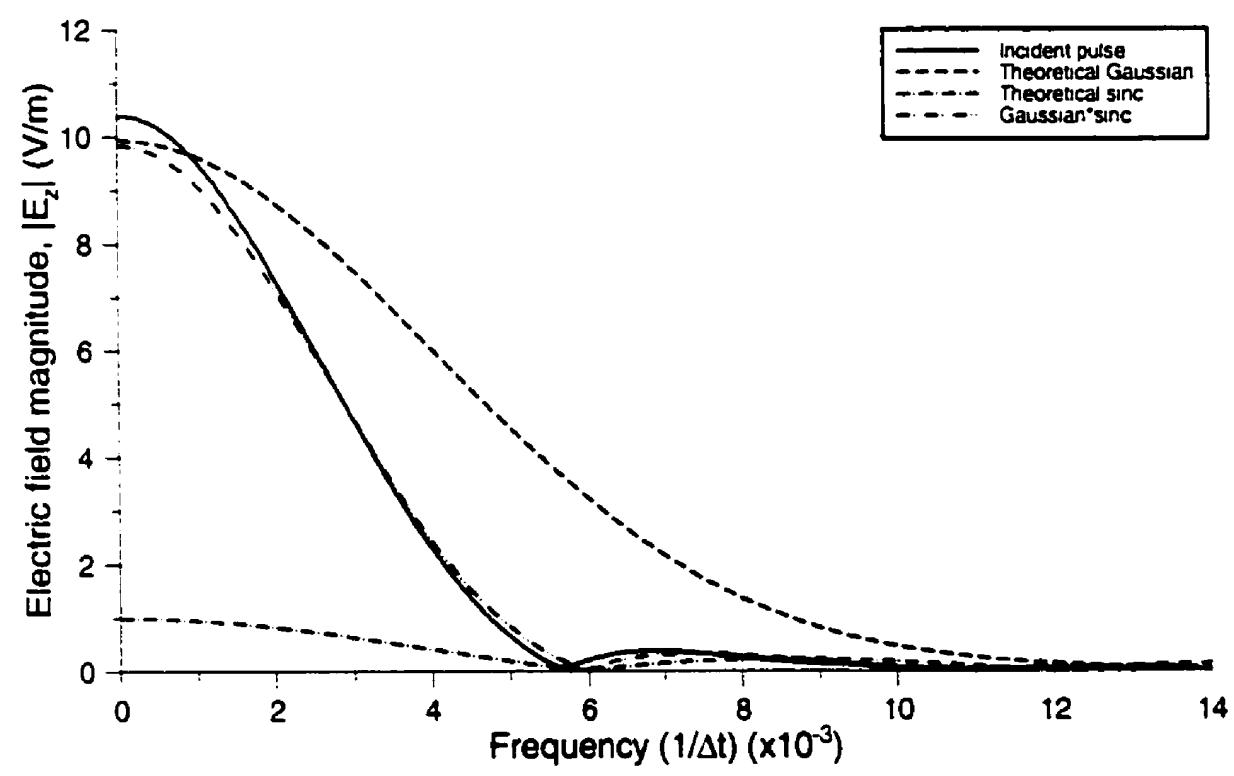

Figure 3.14: $D F T$ of the incident pulse of Figure 3.4 (a) for a four walled PMC mesh with a Gaussian excitation launched at $x_{0}=1.04$. 


\subsubsection{Spatial Averaging}

Further effects on the computation of the DFT are explored with the use of different sampling window dimensions in the time domain. The frequency response for the incident Gaussian observed by square windows of size $(80 \Delta \times 80 \Delta),(120 \Delta X 120 \Delta)$, and (160 X X $160 \Delta$ ), are given in Figure 3.15. It is clear from these curves that the change in dimension of the window has a significant effect on the spectral response. This, however, is to be expected, as a change in the window size affects the width of the gate function involved in the convolution with the Gaussian excitation. Specifically, the larger the width of the gate, the narrower the $\sin (x) /(x)$ function becomes in the frequency domain. The zero crossings approach the origin, reducing the bandwidth of the frequency response, as the $\operatorname{sinc}(x)$ function more closely approximates a true unit impulse function.

To further illustrate the effect of window size on the frequency response of the signal, the DFT computed for each case is shown illustrated with the original Gaussian (calculated value) and the sinc function corresponding to the correct gate function. The product of these two curves is also provided to illustrate their convolution and resulting waveform in the frequency domain. Figures 3.16 (a) to (c) give all these traces for each window case.

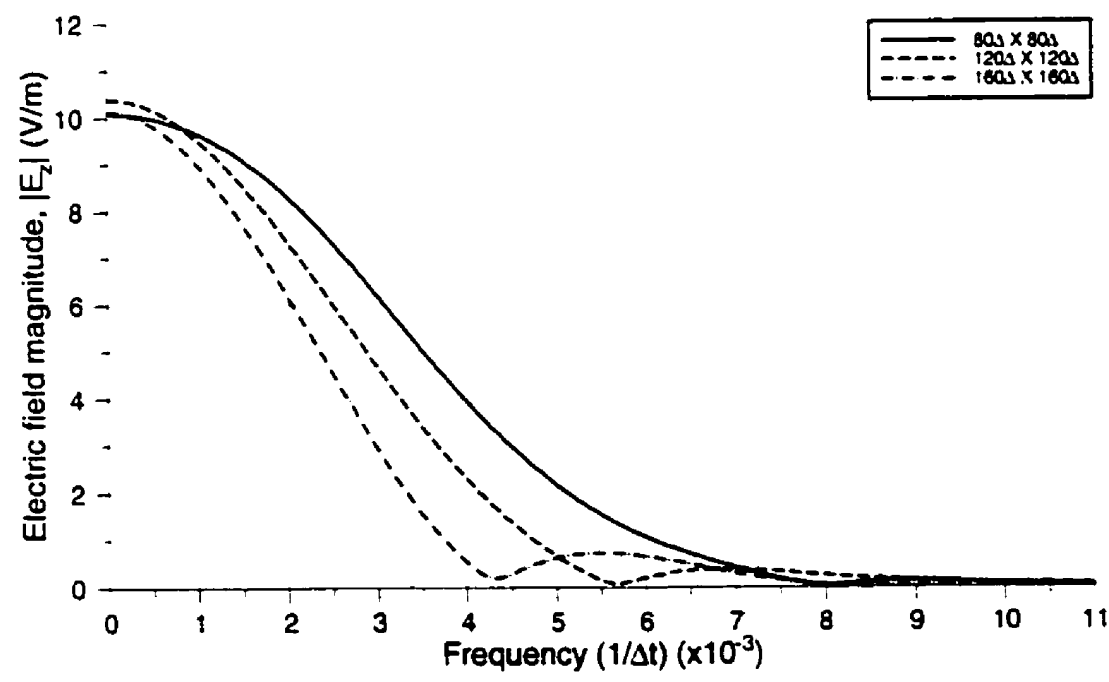

Figure 3.15: DFT of incident Gaussian for simulations of varying window size. 
(a)

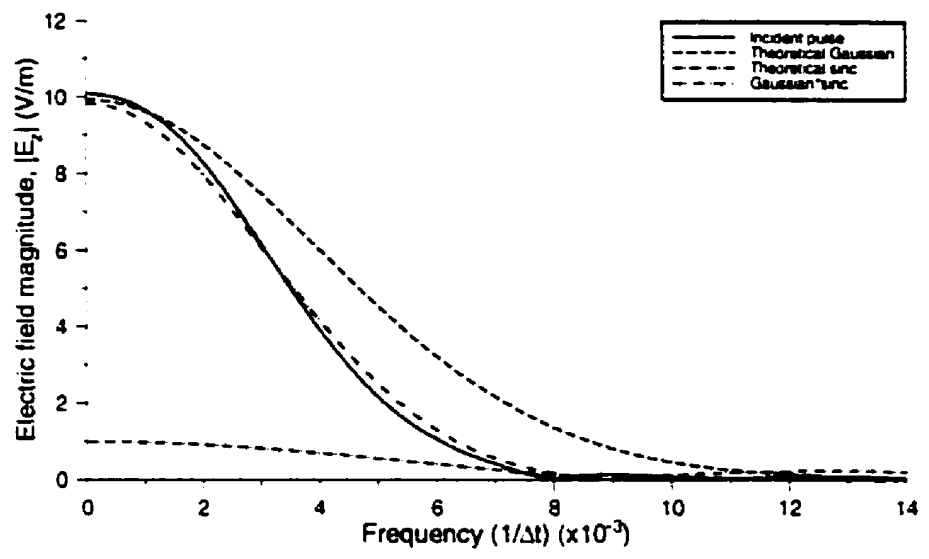

(b)

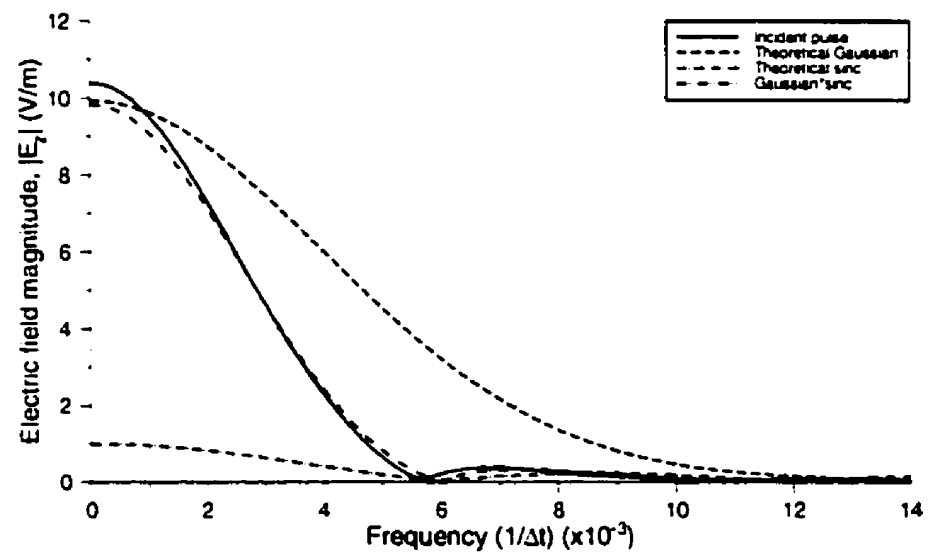

(c)

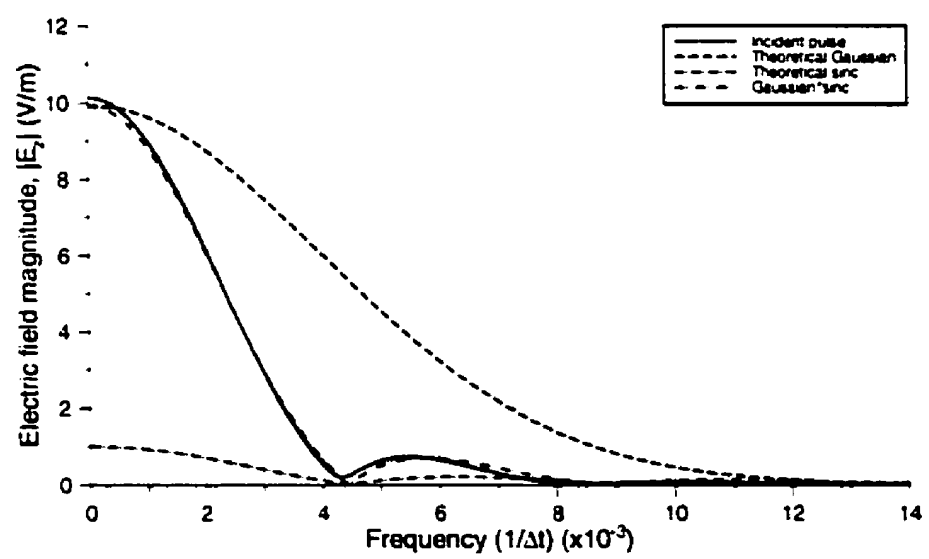

Figure 3.16: DFT of incident LGA Gaussian, theoretical Gaussian and sinc, and their product for windows sizes of (a) 804 X 804, (b) 1204 X 1204, (c) $160 \Delta \times 160 \Delta$. 


\subsubsection{Ensemble Averaging}

The effects of ensemble averaging on the frequency response are shown in Figure 3.17. Using the data presented in section 3.4.2, the DFT of a single simulation, is compared with the DFT of an average of 5, 10, and 20 runs. Their overall responses are very similar in shape and magnitude, with only slight differences in amplitude in and around $F(0)$. The DFT of the 5 run average and the 10 run average are consistent at $F(0)$, while the single run and 20 run DFTs are equivalent at the same point. The single run DFT has a slightly decreased electric field magnitude with respect to the 20 and 10 runs cases, while the ensemble of 5 simulations has a slightly greater magnitude in comparison. The null of the signal is in close agreement in each trace.

A disparity in the field magnitude at $F(0)$ could be explained by the presence of a dc offset. The time domain simulations addressing the rms noise in section 3.4 , as they affect the ensemble averaging, indicated the presence of a dc offset, on the order of $10^{-3}$. However, the effect of this offset in the frequency domain is small. Therefore, the fact that a disparity about the field at $F(0)$ occurred with certain simulation ensembles was regarded as a fluctuation, arising from the particular set of simulation data [31]. 


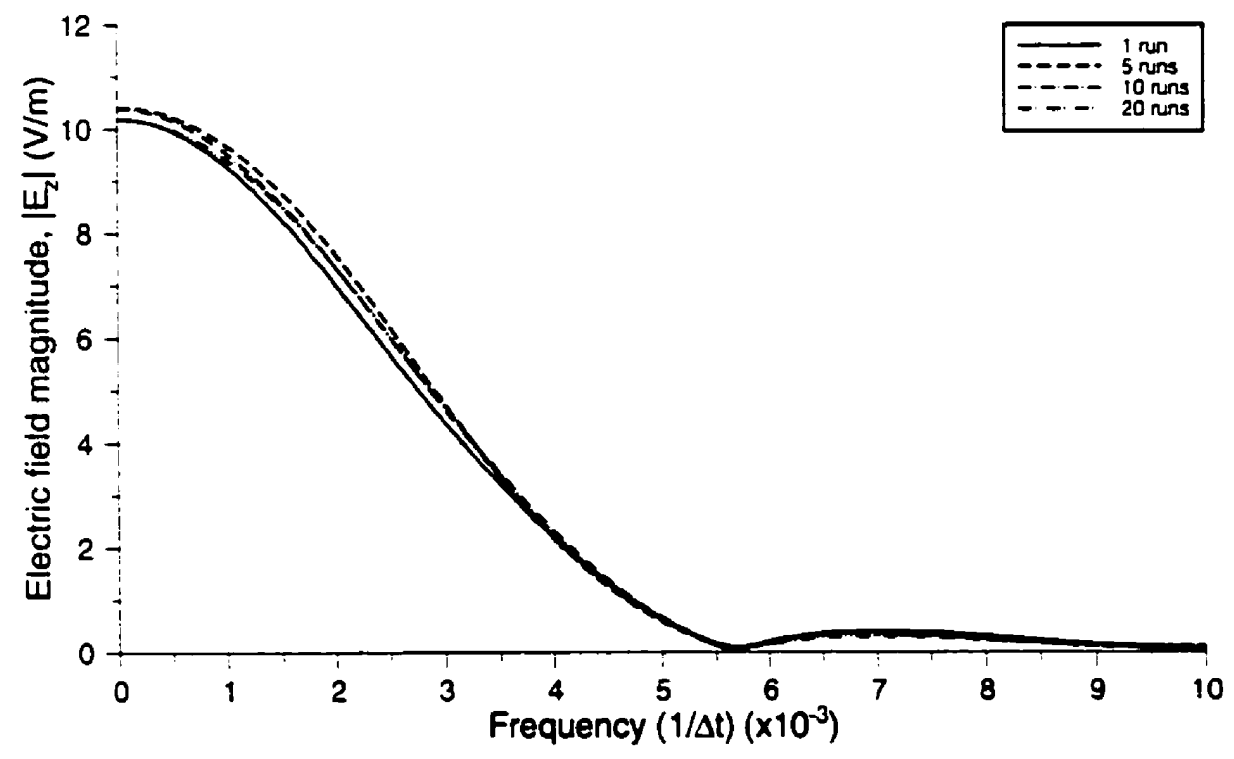

Figure 3.17: DFT of 1 simulation and 5, 10, and 20 simulation averages. 


\subsection{Summary of Results}

Excitation in the LGA lattice was achieved using a Gaussian pulsed plane wave. Due to a comption of the signal in the lattice for higher amplitude levels, perturbation levels are typically $10 \%$ or $20 \%$ above the background distribution of particles. A PMC boundary, simulating an open circuit, is used to initiate a forward travelling Gaussian pulsed plane wave in the mesh. The $A B C$ was shown to have a reflection coefficient of $-15 \mathrm{~dB}$ is adequate for the simulation of a matched load termination.

The effects of viscosity were considered with respect to the propagation velocity and the decay of the pulse perturbation over time. The sound speed in the lattice slows for background particle densities less than approximately 0.4 . The magnitude of the decay constant in (3.6) decreases with increasing $\rho_{o}$. For both the sound speed and pulse decay, these trends are reversed as the background density increased beyond approximately 0.5 . From Figures 3.8 and 3.9, the effects of viscosity on sound speed and decay in the HPP-LGA system are least significant for $0.4 \leq \rho_{0} \leq 0.5$.

The inherent presence of noise in the LGA system can be smoothed by spatial and ensemble averaging. Ensemble averaging was shown to decrease the rms noise level in the system by $\sqrt{N_{\text {ensemble }}}$, and had little effect on the frequency response. However, increasing window size during spatial averaging had the effect of broadening and damping of the pulse, due to the filtering of the signal by the sampling window. Therefore, the dynamic range and accuracy of the macroscopic observable depends on the number of states contributing to the observable and the noise resulting from the averaging process, respectively [2]. The noise level can be reduced by increasing the dimensions of the sampling window, but at the cost of reducing the bandwidth of the frequency response. The square window function undergoes a time domain convolution which results in the product of the Gaussian perturbation and the sinc function. The greater the window, the larger the gate function in the time domain, and the smaller the perturbation bandwidth in the frequency domain. These factors must therefore be considered when selecting a suitable region for averaging. 


\section{Chapter 4}

\section{APPLICATION OF LGA TO SCATTERING PROBLEMS}

This chapter describes experiments simulated using the HPP-LGA algorithm as well as the transmission-line matrix (TLM) method. The basis of the TLM method and the HPPLGA technique are different, yet their implementation by a computer program are strikingly similar. Thus, the data obtained using the LGA technique is compared to that simulated by the TLM method. It should be noted, however, that the performance of the TLM method is not discussed in this thesis. More appropriately, the focus lies on the results of the LGA simulations and their relation to the TLM data.

\subsection{Transmission Line Matrix Method}

The TLM method was introduced in 1971 by Johns and Beurle [32] as a new technique for solving two-dimensional electromagnetic scattering problems, inspired by Huygens' principle for light propagation. The method has been shown to provide an approximate solution to Maxwell's equations in the time-domain [33], and is a recognized tool for the analysis of electromagnetic wave propagation.

\subsubsection{Lumped Element Model of Maxwell's Equations}

A shunt connected node, shown in Figure 4.1 (a), is used to model TM modes. This shunt node is characterized by the intersection of two transmission lines, illustrated in Figure 
4.1 (b), known as the TLM shunt node. These elemental lines make up the TLM mesh, with each node in the mesh separated by the parameter $\Delta l$.

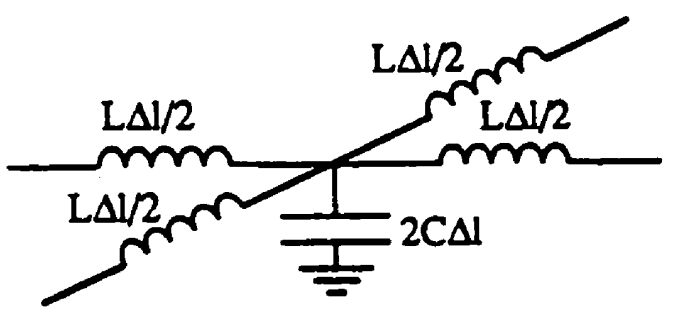

(a)
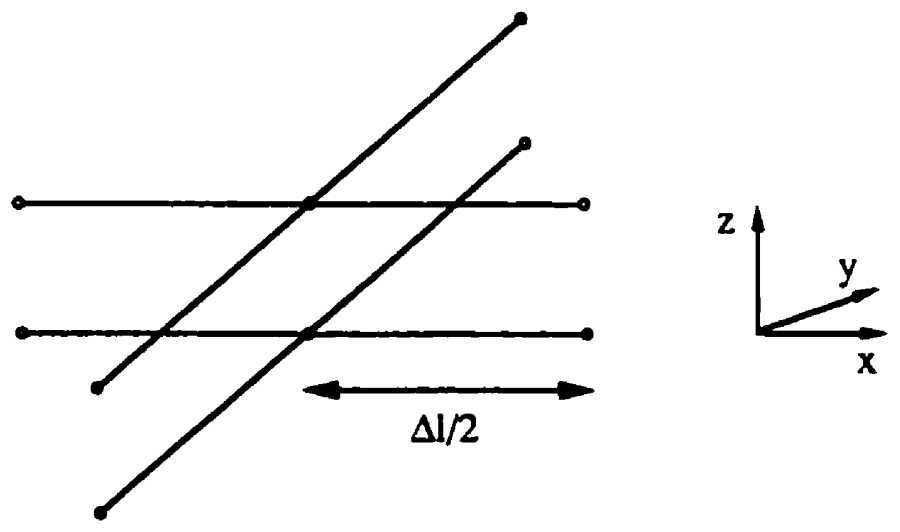

(b)

Figure 4.1: (a) Shunt node and (b) TLM equivalent [33]. 
If only two-dimensional problems are considered, with no field variation in the zdirection, the equivalent field equations for two-dimensional TM propagation can be derived from Maxwell's equations [33,34], by setting $\partial / \partial z=0$, and $H_{z}=E_{x}=E_{y}=0$ to give the following,

$$
\begin{gathered}
\frac{\partial E_{z}}{\partial y}=-\mu \frac{\partial H_{x}}{\partial t} \\
-\frac{\partial E_{z}}{\partial x}=-\mu \frac{\partial H_{y}}{\partial t} \\
\frac{\partial H_{y}}{\partial x}-\frac{\partial H_{x}}{\partial y}=\epsilon \frac{\partial E_{z}}{\partial t}
\end{gathered}
$$

where $\mu$ and $\epsilon$ are the permeability and permittivity of the virtual medium, respectively. Combining equations (4.1) to (4.3) gives the two-dimensional wave equation for the electric field,

$$
\frac{\partial^{2} E_{z}}{\partial x^{2}}-\frac{\partial^{2} E_{z}}{\partial y^{2}}=\mu \epsilon \frac{\partial^{2} E_{z}}{\partial t^{2}}
$$

As long as the mesh parameter $\Delta l$ is much smaller than the shortest wavelength of interest, each mesh element can be considered infinitesimal [35]. Thus, the voltage and current variations in the $x$-and $y$-directions from one node to the next in the network in Figure 4.1 (a) become,

$$
\begin{aligned}
& \frac{\partial V_{z}}{\partial y}=-L \frac{\partial I_{y}}{\partial t} \\
& \frac{\partial V_{z}}{\partial x}=-L \frac{\partial I_{x}}{\partial t}
\end{aligned}
$$




$$
-\frac{\partial I_{x}}{\partial x}-\frac{\partial I_{y}}{\partial y}=2 C \frac{\partial V_{z}}{\partial t}
$$

where $L$ and $C$ are the inductance and capacitance per unit length of an individual line. Combining these equations gives the following two-dimensional wave equation for the voltage in the network,

$$
\frac{\partial^{2} V}{\partial x^{2}}-\frac{\partial^{2} V}{\partial y^{2}}=2 L C \frac{\partial^{2} V_{z}}{\partial t^{2}}
$$

A direct equivalence exists between the voltages and currents in the network and the electric and magnetic fields of Maxwell's equations [32,36]. For a shunt TLM node these relationships are [35],

$$
\begin{gathered}
E_{z} \equiv-V_{z} \\
\mu=L \quad H_{y} \equiv I_{x} \quad \begin{array}{l}
H_{x} \equiv-I_{y} \\
\text { and }
\end{array} \in=2 C
\end{gathered}
$$

Each line section, as in Figure 4.1 (b), possesses the same intrinsic impedance $Z$, and speed of propagation $v$ which are,

$$
\begin{aligned}
& Z_{\text {line }}=\sqrt{\frac{L}{C}} \\
& v_{\text {line }}=\frac{1}{\sqrt{L C}}
\end{aligned}
$$

respectively. The elemental transmission lines of the mesh are considered to represent free space where $L=\mu_{o}$ and $C=\epsilon_{o}$ [33]. However, the medium modelled by the entire mesh of elemental lines is represented by the material properties given in (4.9), where $\mu=L$, and $\epsilon$ 
$=2 C$. Therefore the intrinsic impedance and the speed of propagation of the mesh become,

$$
\begin{aligned}
& Z_{\text {mesh }}=\sqrt{\frac{\mu}{\epsilon}}=\sqrt{\frac{L}{2 C}}=\frac{Z_{o}}{\sqrt{2}} \\
& v_{\text {mesh }}=\frac{1}{\sqrt{\mu \epsilon}}=\frac{1}{\sqrt{2 L C}}=\frac{c_{o}}{\sqrt{2}}
\end{aligned}
$$

respectively, where $c_{o}$ is the speed of light, and $\eta_{o}$ is the intrinsic impedance of free space.

Usually the media properties of the elemental transmission lines are normalized [33]. Thus, $L=1, C=1$, and the media properties of the mesh become $\mu=1, \epsilon=2$. Therefore, (4.12) and (4.13) become,

$$
\begin{aligned}
& Z_{\text {mesh }}=\frac{1}{\sqrt{2}} \\
& v_{\text {mesh }}=\frac{1}{\sqrt{2}}
\end{aligned}
$$

respectively. Equation (4.15) is identical to (3.3) for the speed of propagation in the LGA lattice. 


\subsubsection{TLM Algorithm}

If a voltage impulse is excited at a particular node, as shown in Figure 4.2 (a), the remaining three lines adjoining the node are seen as being in parallel, having an effective impedance of $Z / 3$. The impulse is partially reflected and transmitted at the node, shown in Figure 4.2 (b), according to the following relationships for a I $\mathrm{V}$ pulse,

$$
\begin{aligned}
& \Gamma=\frac{Z / 3-Z}{Z / 3+Z}=-0.5 \\
& \tau=\frac{2(Z / 3)}{Z / 3+Z}=0.5
\end{aligned}
$$

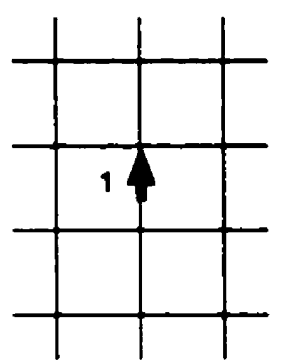

(a)

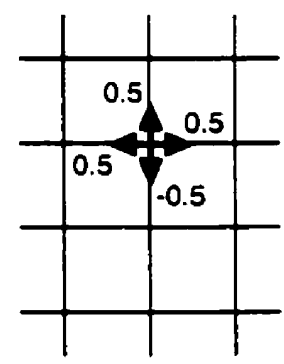

(b)

Figure 4.2: Schematic of a TLM mesh: (a) impulse excitation, (b) results of the first scattering event [34].

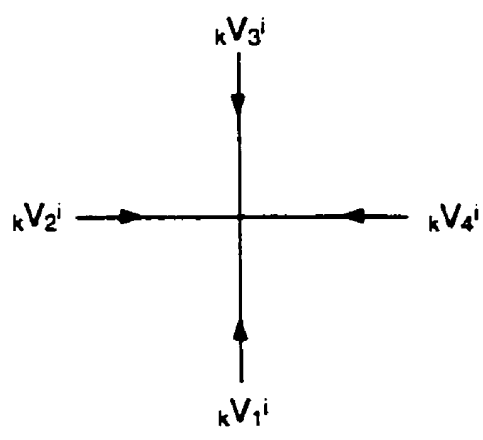

Figure 4.3: Node configuration. 
The scattered impulses continue to propagate through the mesh becoming incident on adjacent nodes and scattering in the manner described above. Each pulse travels from one node to another in one time step $\Delta t$. The incident voltage impulses ${ }_{k} V_{1}^{i},{ }_{k} V_{2}^{i},{ }_{k} V_{3}^{i}$, and ${ }_{k} V_{4}^{i}$, referenced as shown in Figure 4.3, hit the node at time $t=k \Delta t$ along the four lines and are scattered instantly, resulting in four reflected impulses ${ }_{k} V_{1}^{r},{ }_{k} V_{2}^{r}{ }_{k} V_{3}^{r}$, and ${ }_{k} V_{4}^{r}$. The reflected impulses then travel to neighbouring cells to become incident voltages at time $(k+1) \Delta t$. The behaviour of the mesh can be described by a voltage scattering matrix, which relates the reflected voltages at each node to the corresponding incident voltages [35],

$$
\left[\begin{array}{l}
{ }_{k} V_{1}^{r} \\
{ }_{k} V_{2}^{r} \\
{ }_{k} V_{3}^{r} \\
{ }_{k} V_{4}^{r}
\end{array}\right]=0.5\left[\begin{array}{rrrr}
-1 & 1 & 1 & 1 \\
1 & -1 & 1 & 1 \\
1 & 1 & -1 & 1 \\
1 & 1 & 1 & -1
\end{array}\right]\left[\begin{array}{l}
{ }_{k} V_{1}^{i} \\
{ }^{2} V_{2}^{i} \\
{ }_{k} V_{3}^{i} \\
{ }_{k} V_{4}^{i}
\end{array}\right]
$$

A wave can be launched in the TLM mesh by exciting impulses at several nodes. Unlike the LGA method, the TLM method does not require a background distribution of particles.

It is possible to determine the incident voltages at time $(k+1) \Delta t$ at a particular node, since they are related to the reflected pulses at neighbouring nodes. Hence, the new incident voltages on node $(x, y)$ depend entirely on the nodes connected to it and can be expressed by the following node connection equations [34],

$$
\begin{aligned}
& { }_{k-1} V_{1}^{i}(x, y)={ }_{k} V_{3}^{r}(x, y-\Delta l) \\
& { }_{k-1} V_{2}^{i}(x, y)={ }_{k} V_{4}^{r}(x-\Delta l, y) \\
& { }_{k-1} V_{3}^{i}(x, y)={ }_{k} V_{1}^{r}(x, y+\Delta l) \\
& { }_{k-1} V_{4}^{i}(x, y)={ }_{k} V_{2}^{r}(x+\Delta l, y)
\end{aligned}
$$

The voltage, and thus electric field, at a shunt TLM node is determined by the sum of the incident and reflected impulses,

$$
{ }_{k} V_{\text {node }}=\frac{1}{2} \sum_{m=1}^{+}{ }_{k} V_{m}^{i}
$$


For the cases in this chapter, the electric field was calculated using the sampling region method outline in section 2.3.2. The voltage at each node was determined and the averaged voltage in the sampling region $R$ was computed as follows,

$$
V_{\text {ave }}=\frac{1}{N_{T}} \sum_{R}{ }_{k} V_{\text {node }}
$$

where $N_{T}$ represents the total number of possible impulses in the sampling region.

\subsubsection{Boundary Conditions}

The boundary conditions implemented with the TLM method in this thesis, consist of perfect magnetic conducting walls and absorbing boundary conditions. The boundary nodes $(x=i, y=j)$ in the TLM algorithm are computed as [35],

$$
{ }_{k} V_{n}^{r}(i, j)=\Gamma_{i k} V_{n}^{i}(i, j)
$$

where $\Gamma_{t}$ is the impulse reflection coefficient.

\subsubsection{Perfect Magnetic Conducting Boundary}

A perfect magnetic boundary is a lossless non-dispersive boundary, having a bounceback effect on the TLM node impulses. For a shunt-connected network with $V_{z}$ simulating an electric field, a magnetic wall is represented by an open circuit. The boundary is therefore modeled as a reflecting wall of infinite impedance with a reflection coefficient of +1 . An impulse incident at the PMC node at time $k \Delta t$, undergoes a reflection, returning to its original node at $(k+1) \Delta t$ with equal magnitude and phase.

In the TLM algorithm, the PMC boundary can be computed as [35], 


$$
{ }_{k} V_{n}^{r}(i, j)={ }_{k} V_{n}^{i}(i, j)
$$

for each boundary node.

\subsubsection{Absorbing Boundary}

An absorbing boundary condition is a lossy non-dispersive boundary used to simulate matched impedance conditions. The boundary nodes must therefore possess an impedance equal to the intrinsic impedance of the mesh, i.e. $Z_{\text {mesh }}=Z_{o}$. Thus, the impedance of the

elemental lines becomes $Z_{\text {line }}=Z_{o} \sqrt{2}$, and the impulse reflection coefficient seen by the mesh transmission lines is [35],

$$
\Gamma_{i}=\frac{Z_{o}-Z_{o} \sqrt{2}}{Z_{o}-Z_{o} \sqrt{2}}=-0.17157
$$

Therefore, the $\mathrm{ABC}$ boundary is modelled by making the following computation,

$$
{ }_{k} V_{n}^{r}(i, j)=-0.17157_{k} V_{n}^{i}(i, j)
$$

The reflection coefficient is not zero as would be the case for a matched system. Huygens' model for wave propagation describes a series of radiators that emit a wavefront that gives rise to further generations of wavelets in all directions, including towards the back of the radiator. Therefore those wavelets that are projected backward are not influenced by the $A B C$. The TLM method is in essence a discretized form of Huygens' principle and as a consequence, the non-zero reflection coefficient used to implement the absorbing boundary is a direct result of the properties of Huygens' principle of wave propagation. 


\subsection{Scattering Experiments}

A series of scattering experiments were implemented using the HPP-LGA algorithm, and compared to results obtained from the TLM simulations of similar mesh environments. Both methods have a similar implementation in a computer environment, but their fundamental formulations are distinct.

- The TLM method is based on a lumped element model of Maxwell's equations from which a transmission line model is obtained [33]. The HPPLGA method, models physical systems through the interaction and behaviour of particles occupying velocity states within the cells of its lattice.

- $\quad$ Four real number variables occupy each TLM cell. The HPP-LGA particles involve four single bit variables per cell.

- Interactions in the TLM lattice involve floating point multiplication and arithmetic. Particle interaction in the HPP lattice is implemented by table look-up.

- No spatial averaging is required in the TLM algorithm. Spatial averaging is necessary in the HPP-LGA algorithm to obtain the macroscopic observables.

Despite these contrasts, a number of similarities exist in the implementation of both the TLM and HPP-LGA algorithms:

- One time step in the TLM mesh is defined by the scattering of voltage impulses at a node and then the departure of the resulting reflected and transmitted impulses across the borders of the cell in question. With the 
LGA method, one time step is defined by the collision and subsequent advection of particles at a particular cell to its neighbouring cells.

- The reflected and transmitted pulses at a particular node in the TLM mesh following the scattering event, are transferred to four adjacent nodes along their directions of propagation. Following the collision event in the LGA mesh, particles in a particular cell are transferred to four neighbouring cells along their directions of motion.

- The velocity of a propagating wave within a TLM lattice is identical to the sound speed in the HPP-LGA mesh.

Because of these similarities, the TLM method was used to gauge the performance of the HPP-LGA approach for the simulation of electromagnetic phenomena.

As in Figure 4.4, each LGA experiment in this chapter involves the simulation of a scattering event in a two-dimensional $801 \Delta x$ X $801 \Delta y$ lattice, with $\Delta x=\Delta y=\Delta_{L G .1}=0.0005$ $\mathrm{m}$, implemented with PMC boundaries on all four walls. An $x$-directed Gaussian pulse is launched at $x_{o}=1 \Delta_{L G A}$ over a background distribution with a particle density of 0.5 . The pulse has a width of $\sigma=40 \Delta_{L G A}$ and an amplitude $20 \%$ above the background particle distribution. Two windows are placed in the mesh, centered at $\left(x=321 \Delta_{L G . t}, y=401 \Delta_{L G .}\right)$, and at $\left(x=621 \Delta_{L G .}, y=401 \Delta_{L G A}\right)$. A rectangular cylinder was placed in the mesh between the two sampling windows. The four walls of the cylinder cross-section are assigned PMC boundaries so that HPP-LGA particles bounce-back upon contact. The time domain waveforms shown in this chapter are the result of an average of 10 simulations with different random number generator seeds. The pulse is allowed to propagate for $1600 \Delta t$ iterations. The lattice in these experiments simulates a TM cavity, where the macroscopic density in each sampling window computes $E_{z}$ at every time step.

The experimental mesh was scaled to ease the computation of the TLM simulations. From previous experiments $[7,8]$, we have found mesh densities of $\Delta l_{T L}=10 \Delta l_{L G A}$ to be 
adequate. The mesh was initialized with dimensions of $201 \Delta x \times 201 \Delta y$, with the lattice inter-nodal spacing taken as $\Delta x=\Delta y=\Delta_{T L u}=0.002 \mathrm{~m}$. Thus, the cell size is scaled by a factor of 4 , and the overall mesh size is 16 times smaller than the implemented LGA mesh. A Gaussian wave of amplitude 0.2 was launched at $x_{o}=1 \Delta_{T L}$ with a width of $\sigma=10 \Delta_{T L M}$ for a time of $400 \Delta t$. The cross-sectional area of the rectangular cylinder, and their mesh coordinates have also been scaled by a factor of 4 . Figure 4.4 shows the mesh parameters used for the LGA and TLM simulations.

(a)
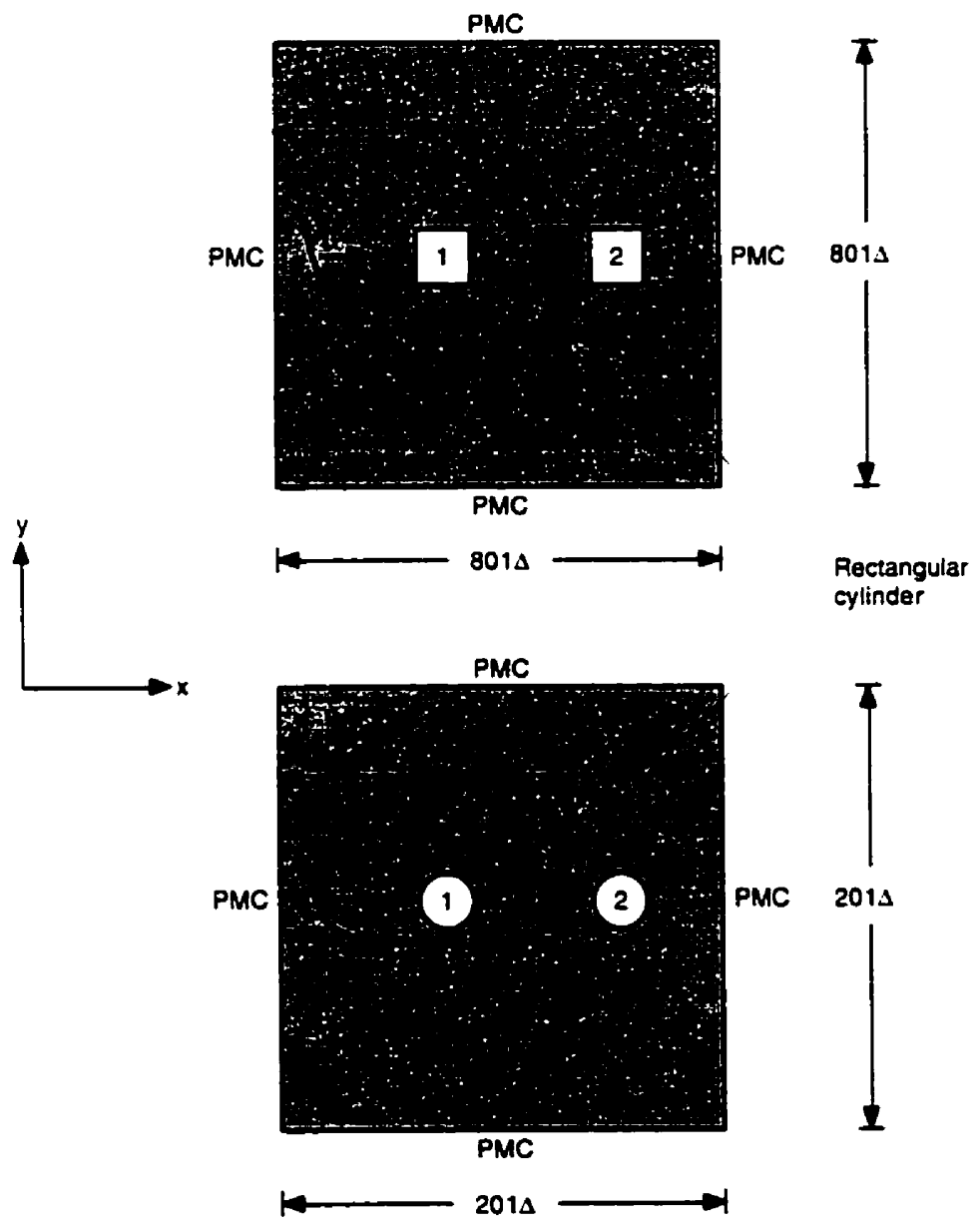

(b)

Figure 4.4: (a) LGA lattice, (b) TLM lattice, (c) physical cavity dimensions, with rectangular cylinder cross-section and two sampling windows. 
(c)

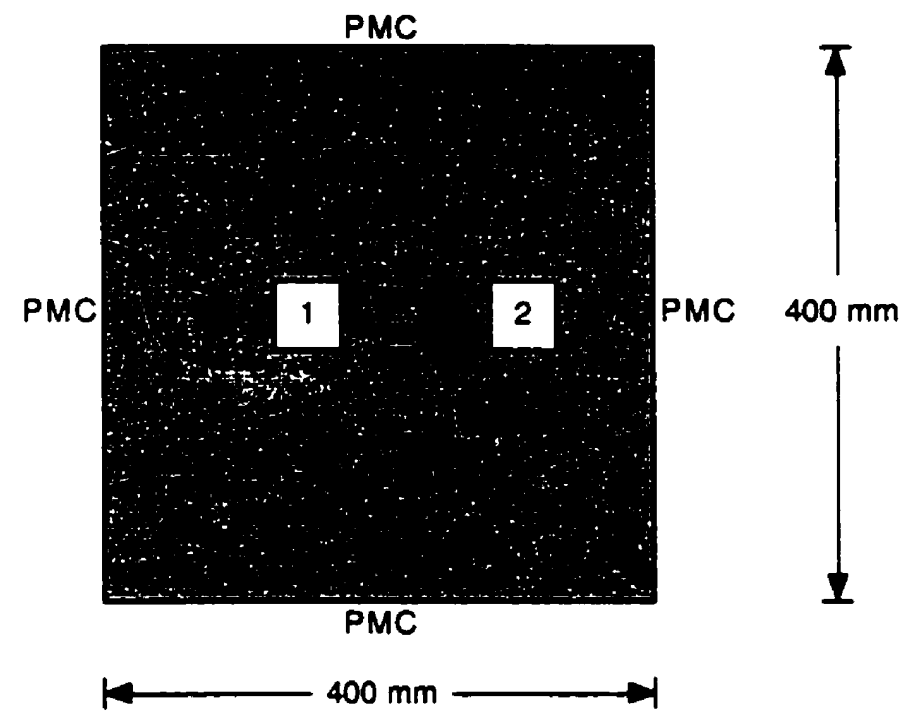

Figure 4.4 (continued)

The LGA and TLM lattice configurations correspond to the physical dimensions detailed in Figure 4.4 (c). The rectangular cylinder has a cross-sectional area of $30 \mathrm{~mm} \mathrm{X}$ $60 \mathrm{~mm}$. within a $400 \mathrm{~mm} \times 400 \mathrm{~mm}$ square PMC cavity. Each time step $\Delta t$, is on the order of $0.001 \mathrm{nsec}$ in free space, normalized by the speed of light in vacuum, $3 \times 10^{8} \mathrm{~m} / \mathrm{s}$. 


\subsubsection{Effect of Window Size on Scattered Signals}

A rectangular cylinder with a cross-sectional area of $60 \Delta_{L G A} \times 120 \Delta_{L G A}$ is placed in the mesh, as shown in Figure 4.4. The square sampling windows used throughout Chapter 3 were considered satisfactory for the scattering experiments in this chapter, since only normally incident Gaussian pulsed plane waves were propagated in the lattice. Three different window sizes were utilized to compute the electric field at each time step:

$$
\begin{array}{ll}
\text { Case 1: } & 80 \Delta_{L G A} \times 80 \Delta_{L G A} \\
\text { Case 2: } & 120 \Delta_{L G A} \times 120 \Delta_{L G A} \\
\text { Case 3: } & 160 \Delta_{L G A} \times 160 \Delta_{L G A}
\end{array}
$$

For problems involving non-normal incidence or edge-diffraction, a circular sampling window would provide an unbiased representation of the perturbation, as its boundaries do not discriminate against a particular direction. A circular window was assigned for the TLM sampling region. It is necessary to consider the weighting of the window dimensions in the direction of pulse propagation, when considering two different sampling geometries. The weighting of the circular region must therefore be analogous to the square sampling window. If the equivalent width of the TLM circular window is less than the LGA window, discrepancies in the pulse amplitude may result, due to the averaging of a smaller number of cells in the window. The required weighting of the diameter of the circular window can be determined with reference to Figure 4.5. The equivalent radius of the TLM circular window can be expressed as follows,

$$
\int_{-R}^{R} \rho w(x) d x=\frac{4}{\pi R^{2}} \int_{-R}^{R} R^{2}-x^{2} d x
$$

where $w(x)$ represents the width of the sampling window at location $x$, and $\rho$ represents the ratio of $w(x)$ to the circular window area $\pi R^{2}$. 


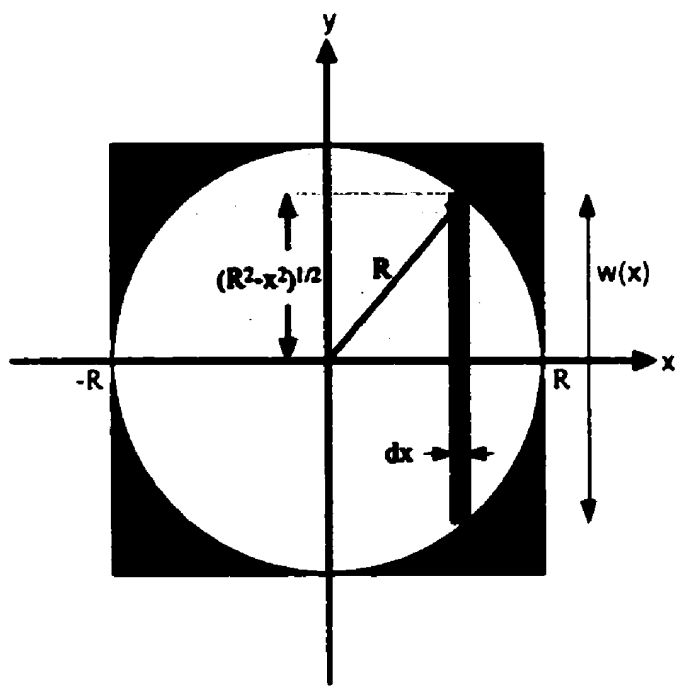

Figure 4.5: Mathematical model for calculating the equivalent circular diameter.

From 4.27, the equivalent diameters for the TLM circular windows for the three LGA cases are:

$\begin{array}{ll}\text { Case 1: } & 94 \Delta_{L G A} \\ \text { Case 2: } & 141 \Delta_{L G A} \\ \text { Case 3: } & 188 \Delta_{L G A}\end{array}$

The above parameters were scaled during simulations, with the circular windows having radii of $12 \Delta_{T L M}, 18 \Delta_{T L U}$, and $24 \Delta_{T L M}$. 


\subsubsection{Time Domain}

A simulation was first performed for identical mesh parameters, but without the scattering object, to compare the results of the two methods. They are shown in Figures 4.6 and 4.7, for sampling windows $I$ and 2 , respectively. The results are nearly the same for the two methods, but the LGA traces show slight dissipation and pulse broadening. This is more prominent for the $80 \times 80$ window case, but is also apparent in all three plots of Figure 4.7. The LGA waveforms are somewhat coarse as a result of noise inherent in the HPP-LGA system. By comparison, the TLM traces are smooth.

The time-domain waveforms of both the LGA and TLM scattering simulations, for the three sampling region sizes, are shown in Figures 4.8 (a) to (c) for window 1, and Figures 4.9 (a) to (c) for window 2. For the field computed by window 1, only the incident Gaussian at $t=440 \Delta t$ is common in both the non-cylinder and cylinder waveforms of Figures 4.6 and 4.8. The results from the second sampling window in Figure 4.9, illustrate slight differences in comparison to Figure 4.7, due to the forward scattering by the object. In each case, the overall trends of both the TLM and LGA waveforms are similar.

Comparing the LGA traces with each other, the broadening of the wave is also noticeable with a sampling area increase. The resulting waveform aiso illustrates a slightly higher degree of noise with a decrease in window dimensions. As addressed in section 3.4, the differences in noise level, and pulse amplitude and width can be attributed to the effects of the smaller sampling region used to calculate the electric field at both locations in the mesh. 
(a)

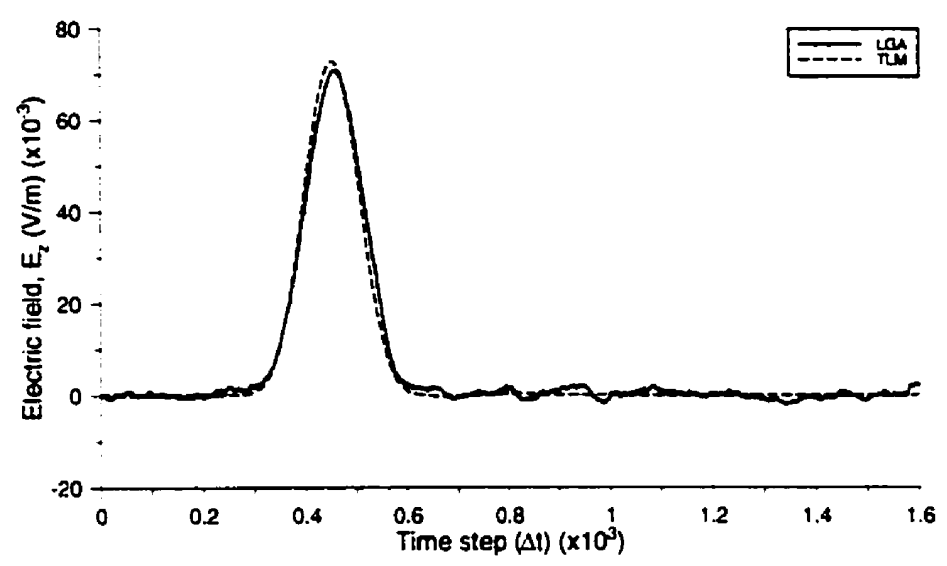

(b)

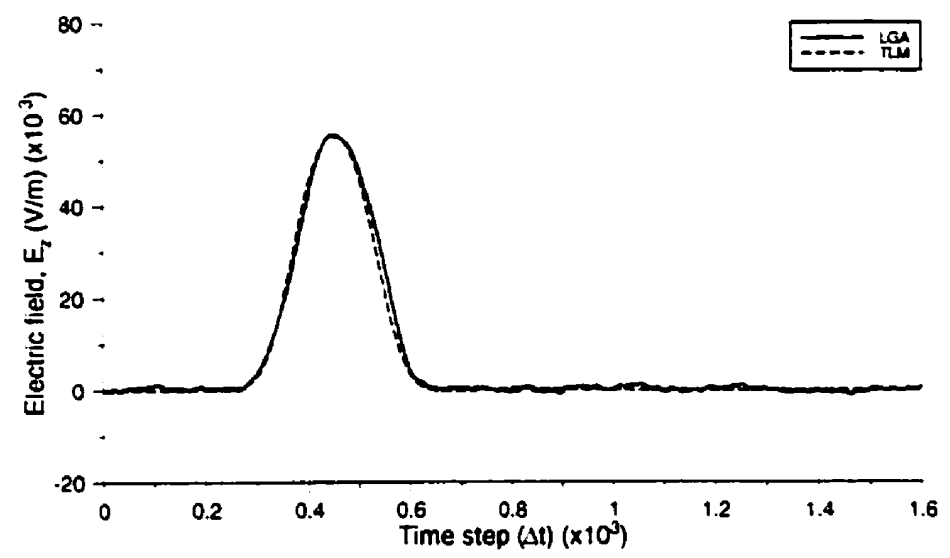

(c)

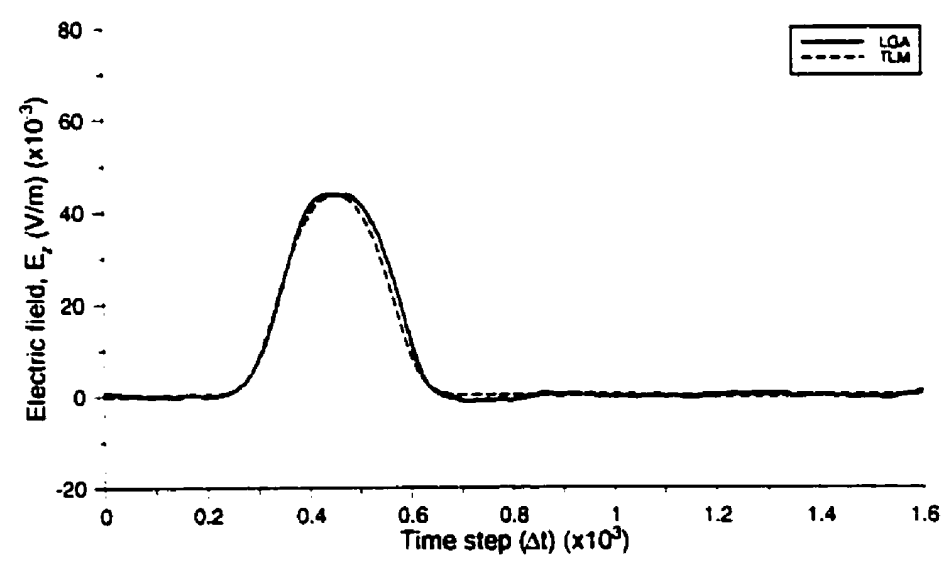

Figure 4.6: Time domain waveforms of window I without scattering object for window dimensions of (a) $80 \Delta \times 80 \Delta$, (b) $120 \Delta \times 1204$, and (c) $160 \Delta \times 160 \Delta$. 
(a)

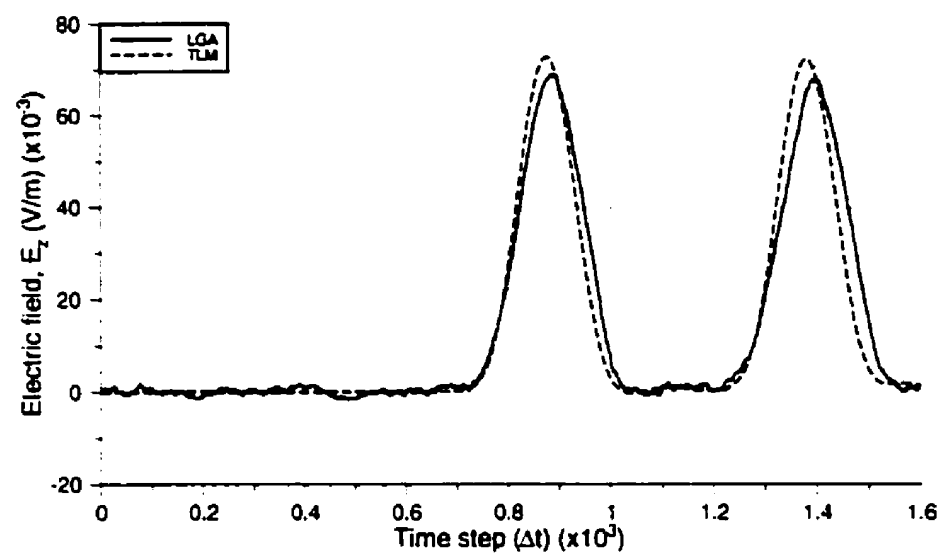

(b)

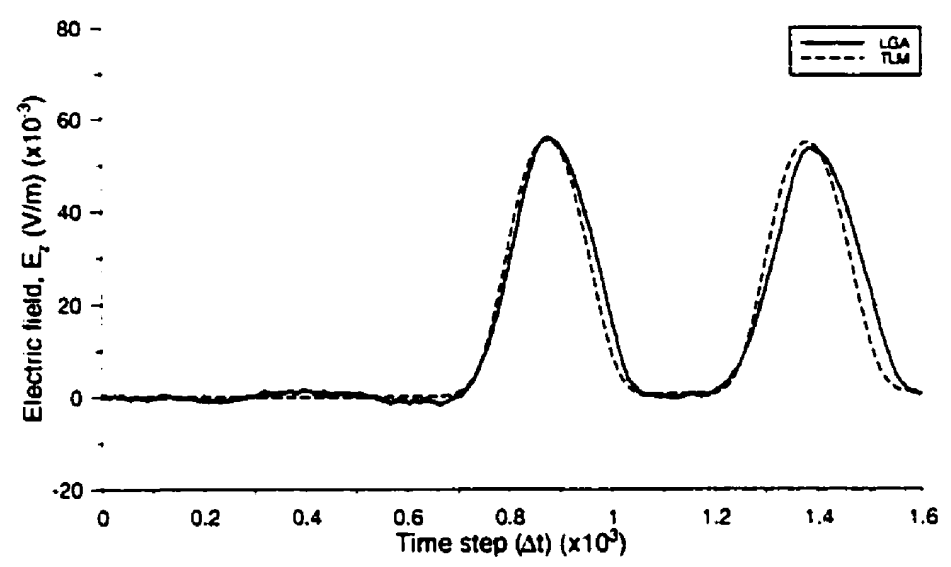

(c)

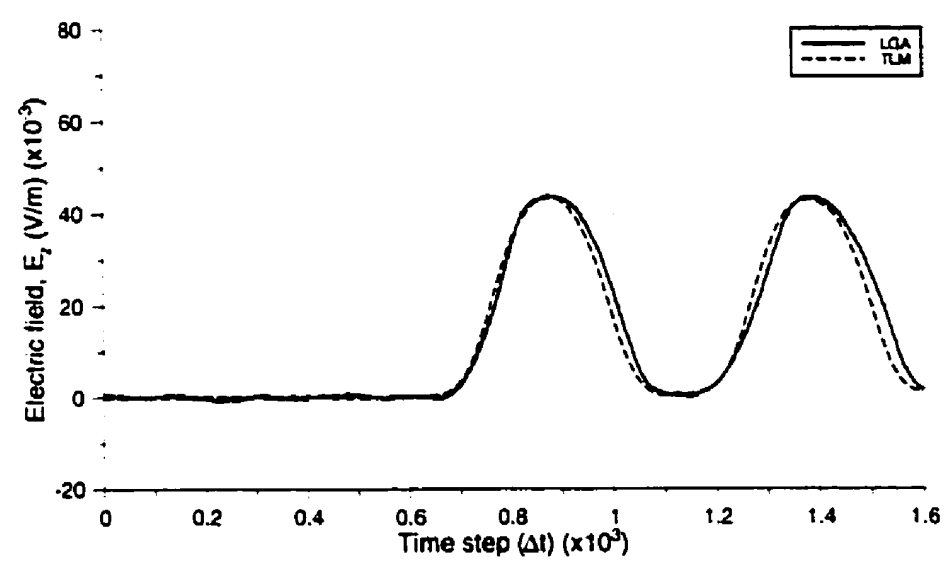

Figure 4.7: Time domain waveforms of window 2 without scattering object for window dimensions of (a) $804 X 804$, (b) $1204 X 1204$, and (c) $160 \Delta \times 160 \Delta$. 
(a)

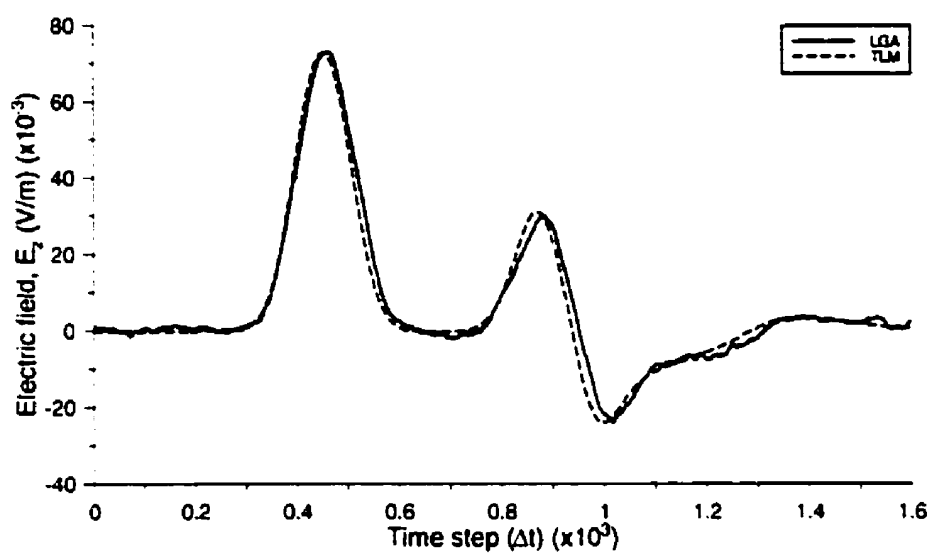

(b)

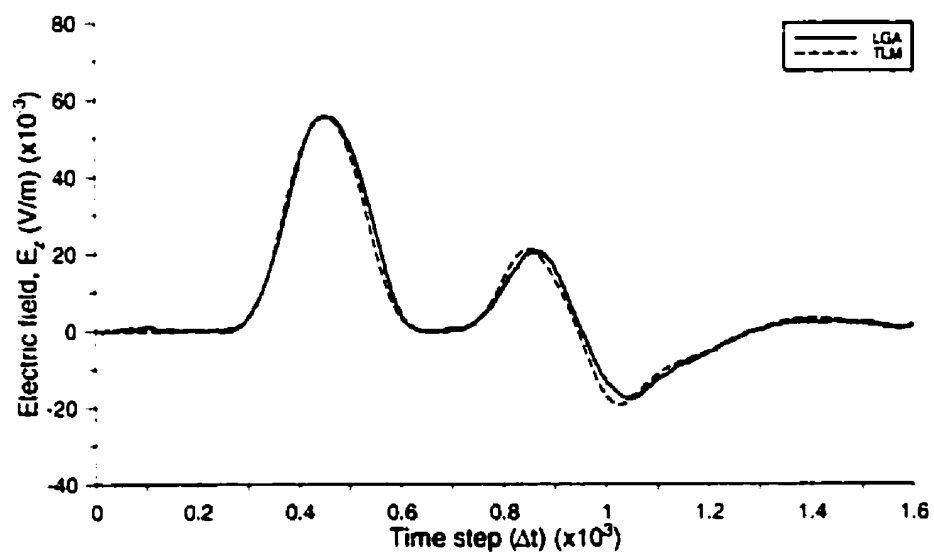

(c)

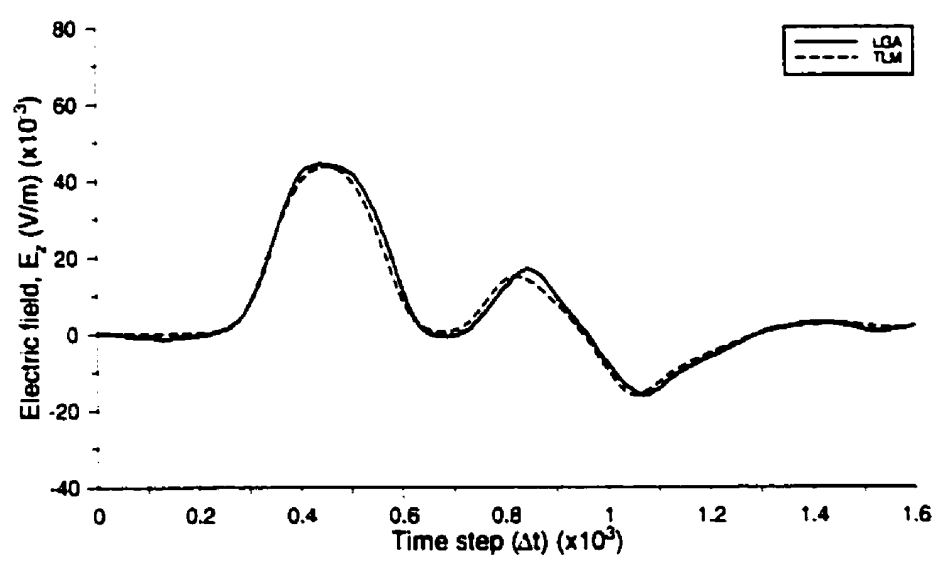

Figure 4.8: Time domain waveforms of window 1 for $60 \Delta X 120 \Delta$ cylinder crosssectional area computed with window dimensions of (a) $80 \Delta X 80 \Delta$, (b) $120 \Delta \times 1204$, and (c) $160 \Delta \times 1604$. 
(a)

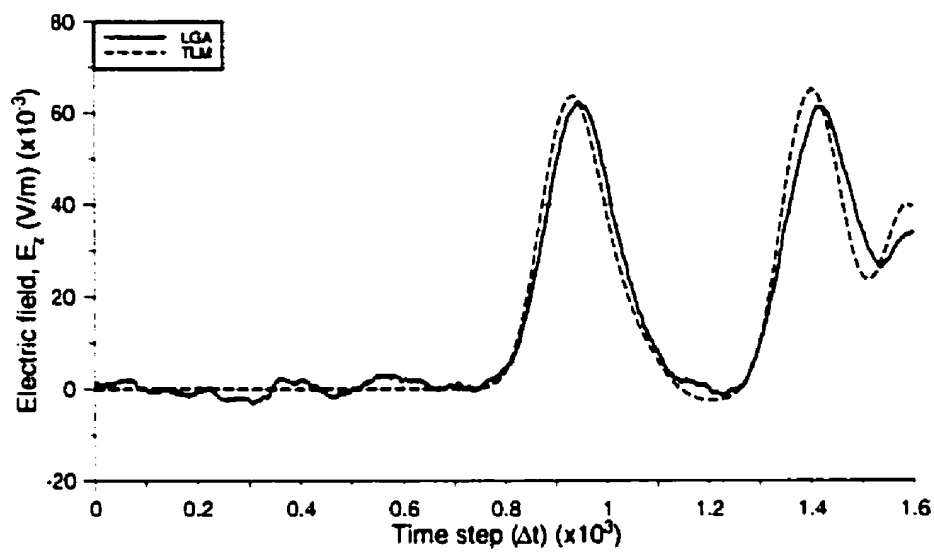

(b)

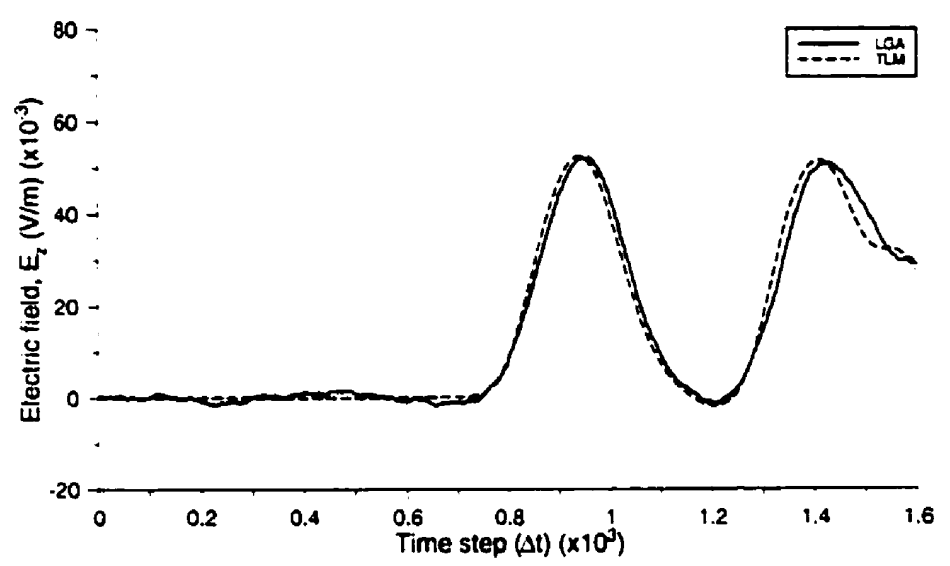

(c)

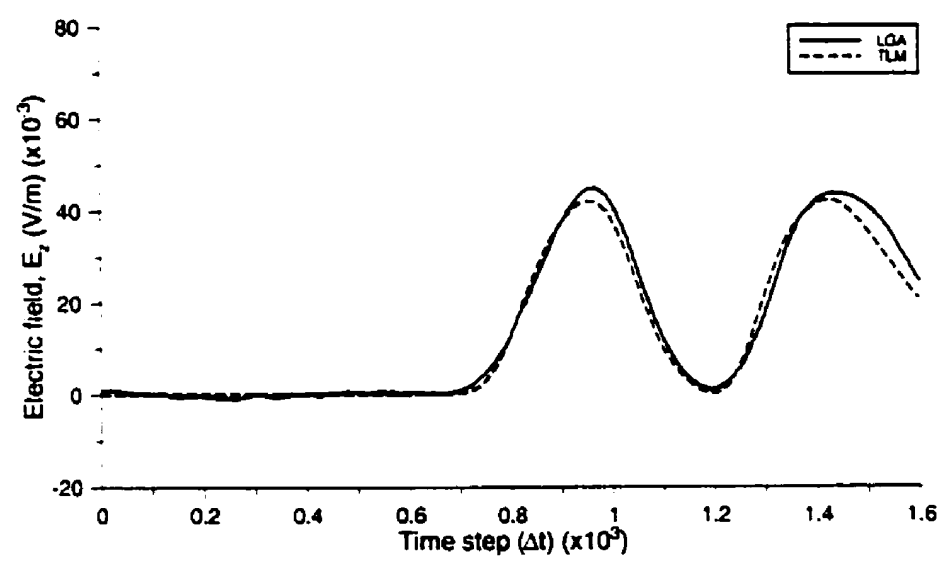

Figure 4.9: Time domain waveforms of window 2 for $60 \Delta X 1204$ cylinder crosssectional area computed with window dimensions of (a) 804X 804. (b) $120 \Delta X 1204$, and (c) $160 \Delta X 160 \Delta$. 
To obtain the forward and backward scattered data, the simulation data obtained without the scattering object was subtracted from the output of the scattered data. These waveforms, shown in Figures 4.10 and 4.11, exhibit the backward and forward scatter of the wave off the object, as observed by window 1 and 2 , respectively. As with the original full time domain output (incident + scatter) in Figures 4.6 and 4.7, the forward and backward scatter waveforms are narrower with larger peaks and deeper nulls as the window sizes decrease. Beyond $t=1387 \Delta t$, the effects due to the east PMC wall are registered in window 2.

The small pulse shown at $t=844 \Delta t$ in Figure 4.10, represents the backward scattered portion of the field by the object in the negative $x$-direction. In contrast, the waveform obtained by window 2 details a decline in the electric field at approximately the same time, at $t=822 \Delta t$ caused by the shadow of the object. The pulse at $t=1038 \Delta t$ in window 2 of Figure 4.11, represents the forward scattered wave that propagated beyond the object in the positive $x$-direction of the lattice. Similarly, a decline in the electric field at $t=1078 \Delta t$ is shown in window 1. 
(a)

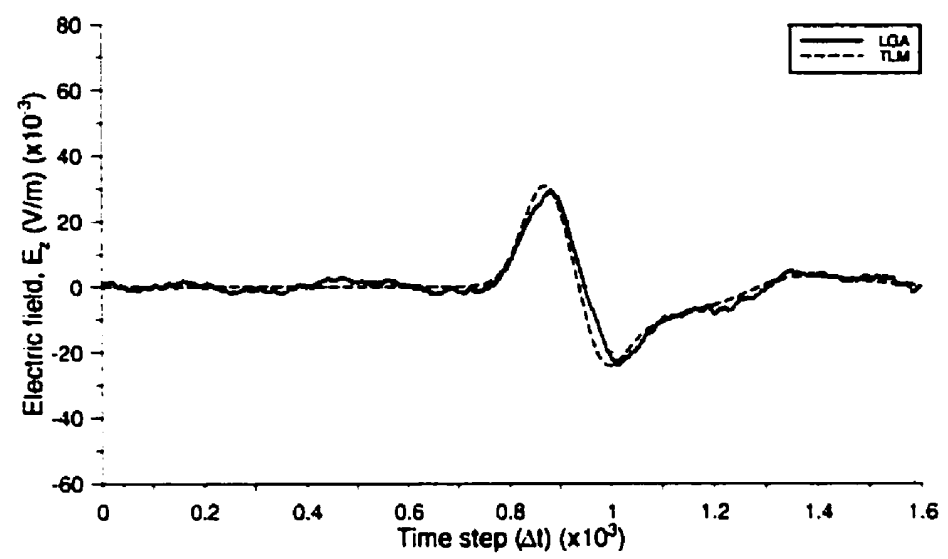

(b)

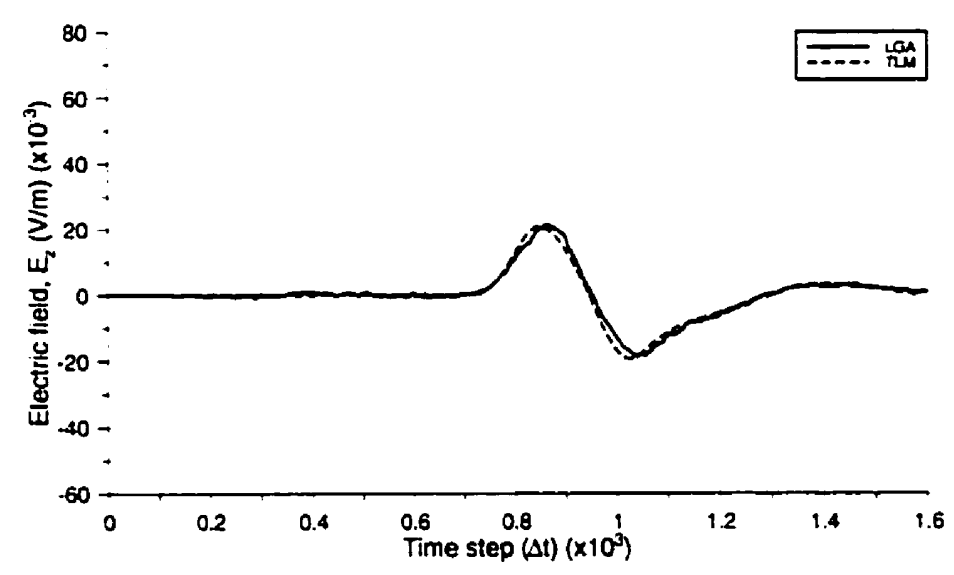

(c)

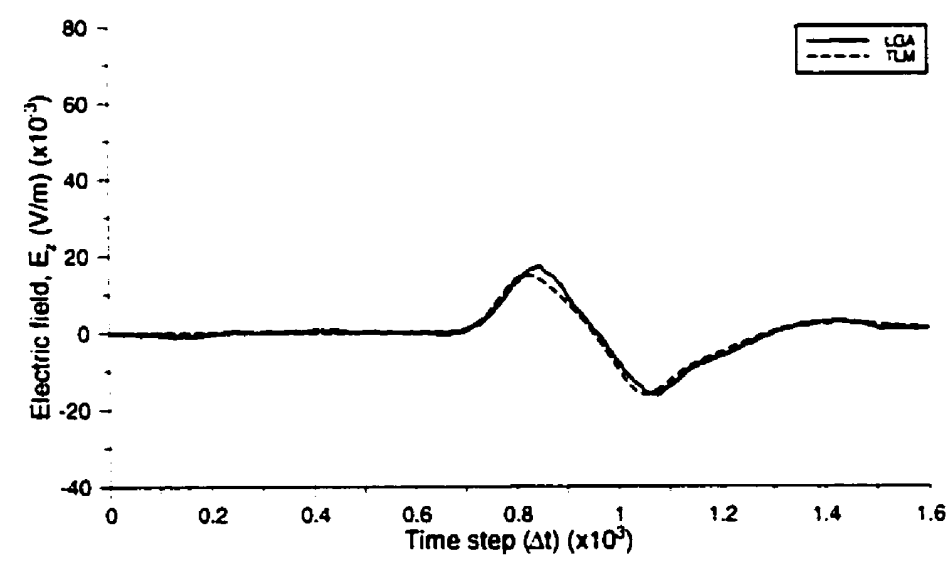

Figure 4.10: Backward scatter at window 1 for 604 X 1204 cylinder crosssectional area computed in the time domain with window dimensions of (a) $80 \Delta X 80 \Delta$, (b) $120 \Delta X 120 \Delta$, and (c) $160 \Delta X 160 \Delta$. 
(a)

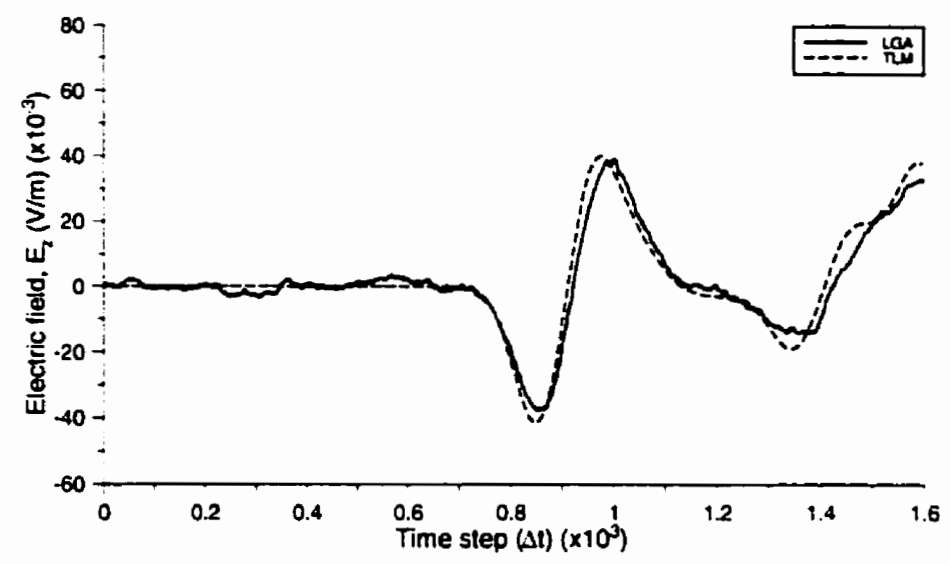

(b)

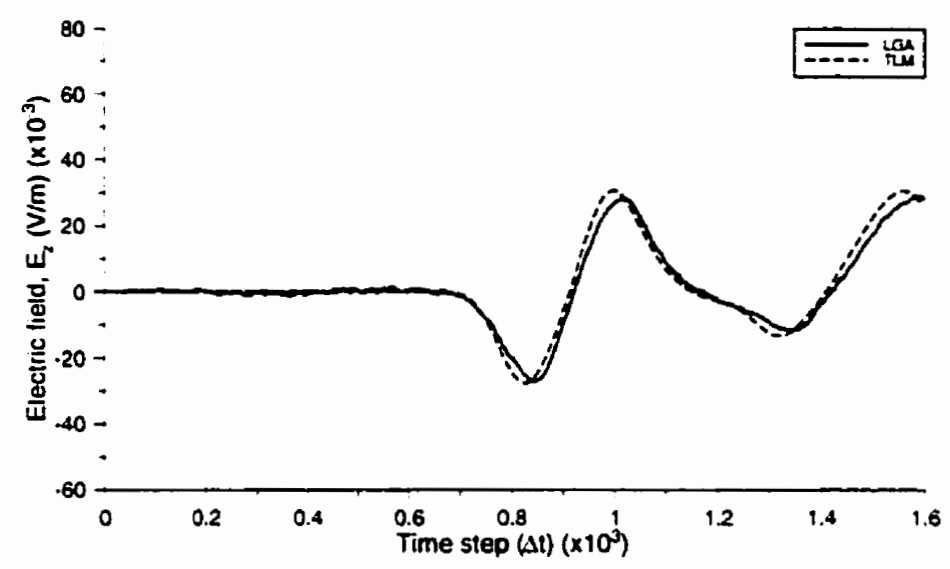

(c)

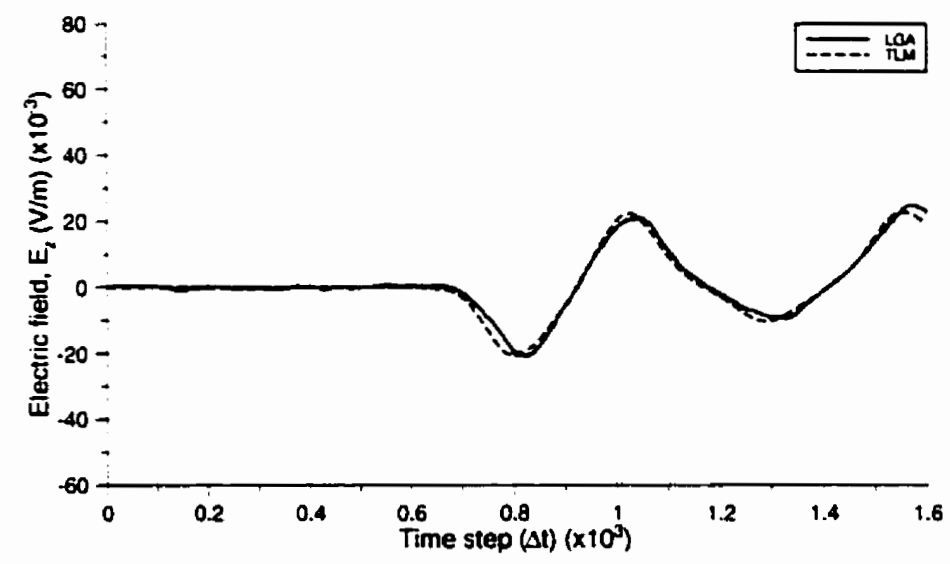

Figure 4.11: Forward scatter at window 2 for $60 \Delta \times 120 \Delta$ cylinder crosssectional area computed in the time domain with window dimensions of (a) $80 \Delta X 804$, (b) $120 \Delta X 1204$, and (c) $160 \Delta \times 1604$. 


\subsubsection{Frequency Domain}

It was discussed in section 3.5, that the square sampling window used in LGA simulations causes the time domain convolution of the gate function with the Gaussian excitation. A similar phenomena develops with the circular sampling window used in TLM simulations.

The time domain circular gate function $f(x, y)$, is defined by $x$ and $y$ Cartesian coordinates, and its Fourier transform $F(v, w)$, by a coordinate system labeled $v$ and $w$, respectively. A radial vector $\rho$ can be defined as $\rho^{2}=x^{2}+y^{2}$, where $f(x, y)=f(\rho)$, allowing for the more convenient integration of the transform using polar coordinates $\rho$ and $\phi$. By circular symmetry, $q^{2}=v^{2}+w^{2}$, where $F(v, w)=F(q)$ in the frequency domain. However, unlike the transform of the square sampling window in which the $x$-and $y$ integrals were independent of one another, the $\rho$ and $\phi$ integrals are not, and thus neither can be neglected. After carrying out the integration, the Fourier transform of a two-dimensional circular aperture becomes [37,38],

$$
F(u)=2 \pi a^{2} \frac{J_{1}(u)}{u}
$$

where $a$ is the radius of the circle, $u=2 \pi a q$, and $J_{I}(u)$ is the first order Bessel function.

The normalized transform of the square sampling window (gate function) and the circular window are shown in Figure 4.12 (a), for identical window widths/diameters. Note that the responses do not agree. The LGA window details a smaller bandwidth as a result of its larger window size. This further illustrates the necessity of determining the correct weighting of the width between two differing window geometries. Figure 4.12 (b) gives the normalized transform of the LGA window and the circular window of equivalent width (referring to the window description by equation (4.27) and Figure 4.5). In this case, the transforms are similar. The magnitude of the peak following the transform nulls is greater for the sinc function. 


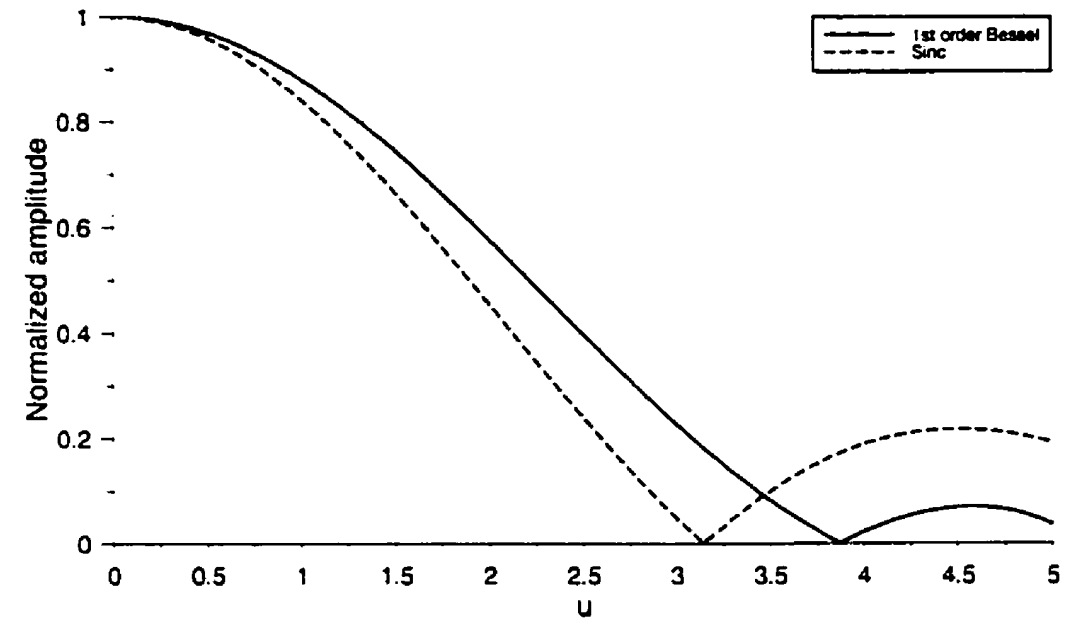

(a)

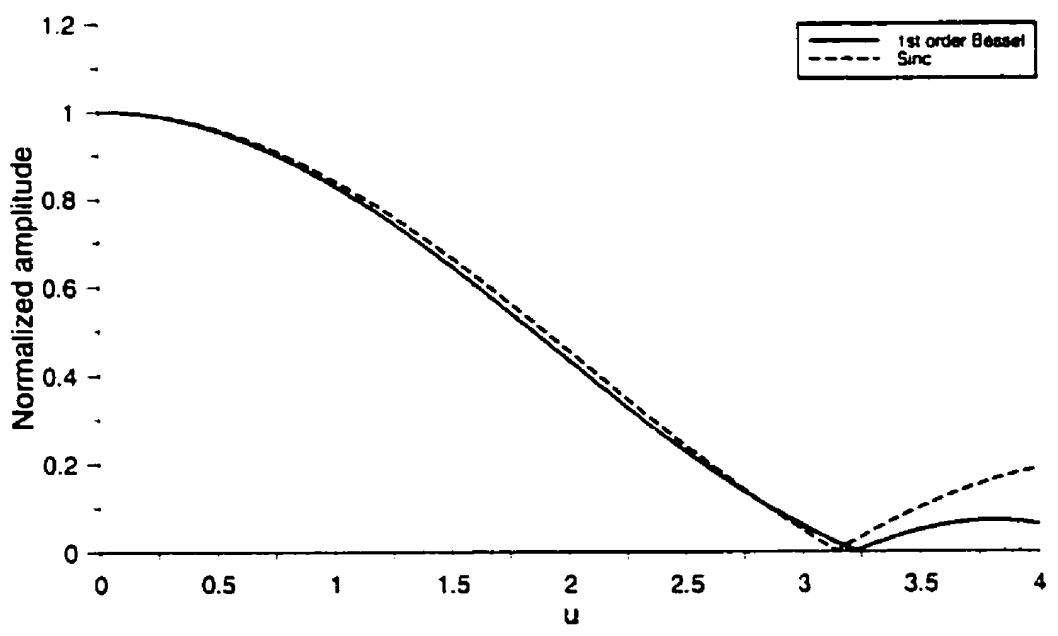

(b)

Figure 4.12: Normalized Fourier transform of a rectangular gate and a circular gate function of (a) identical widths, and (b) with equivalent widths. 
The TLM and LGA frequency response for the incident wave on the object are shown in Figure 4.13. These plots correspond to the time-domain incident fields in Figure 4.6. The frequency response of the scattered cases in Figures 4.8 and 4.9, are shown in Figure 4.14 and 4.15 for windows $I$ and 2 , respectively. These traces were obtained by applying the DFT to the time domain waveforms in Figures 4.8 and 4.9, and include both the incident and scattered field. The LGA and TLM traces for both window 1 and 2 are almost identical for the two methods.

With respect to differences observed due to the size of the sampling windows, the peaks and nulls in Figures 4.14 and 4.15 occur at the same frequencies in all three cases. However, with increasing frequency, the peak field levels have a lesser magnitude for simulations with larger window sizes. This gives the appearance of additional resonant frequencies for smaller windows. Furthermore, the response covers a larger frequency range with smaller window sizes. As discussed in section 3.5, this is due to the widening of the sinc function resulting from the narrowing of the window gate function. Each window configuration was therefore judged to be usable only to frequencies below $2.5 \mathrm{GHz}$ for the $160 \Delta \times 160 \Delta$ window, $3.5 \mathrm{GHz}$ for the $120 \Delta \times 120 \Delta$ window, and $4.0 \mathrm{GHz}$ for the $80 \Delta \mathrm{X}$ $80 \Delta$.

The Fourier transforms of the forward and backward scattered waves are given in Figures 4.16 and 4.17. Prior to taking the DFT of the forward scattered time-domain data, the waveforms were truncated so that only the information present as a direct result of the scattering by the cylinder is computed by the DFT. The reflected pulse by the east PMC wall observed by window 2 was also not considered in the transform of the forward scatter data. The field distribution involving the forward scatter in both Figures 4.15 and 17, shows a greater magnitude in comparison to the field levels observed for the backward scatter in Figures 4.14 and 4.16. Had the scattering object been infinitely thin, the field distribution would have detailed similar patterns in both the forward and backward direction. 
(a)

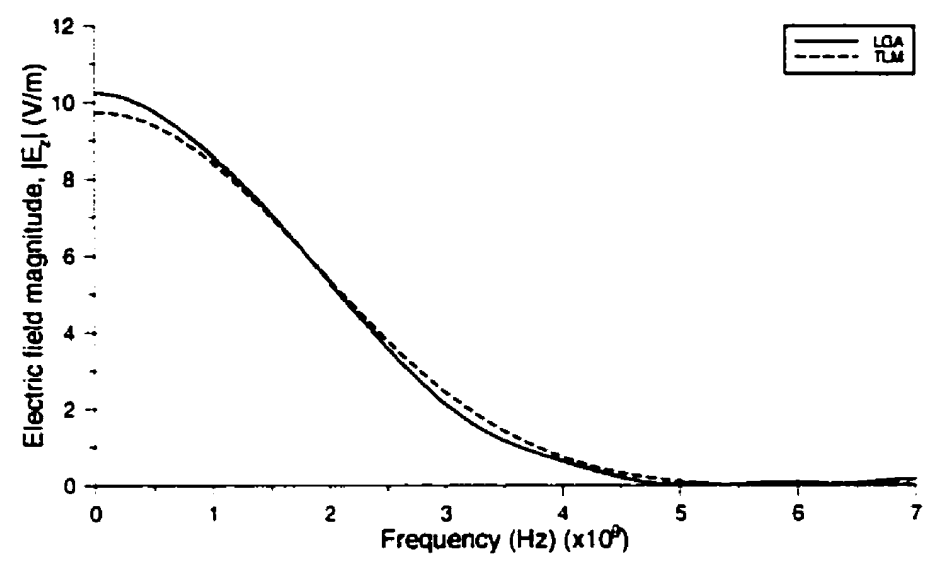

(b)

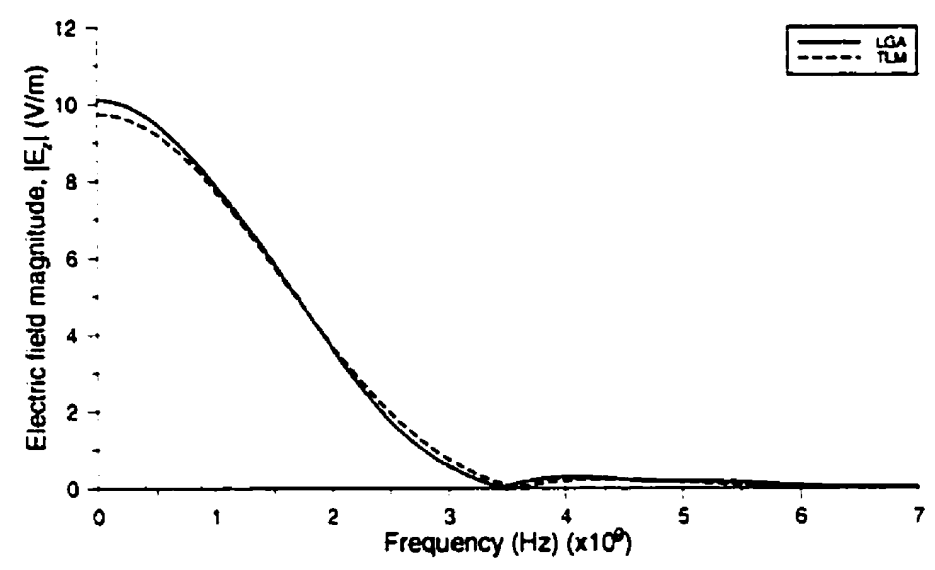

(c)

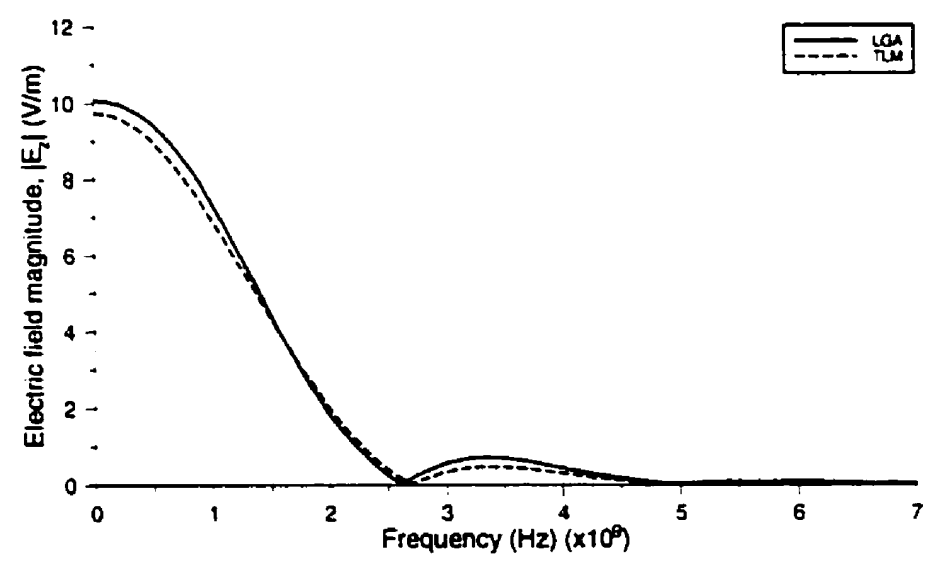

Figure 4.13: Frequency response of the incident pulse computed with window dimensions of (a) $80 \Delta X 804$, (b) $120 \Delta X$ 1204, and (c) $160 \Delta X$ $160 \Delta$. 
(a)

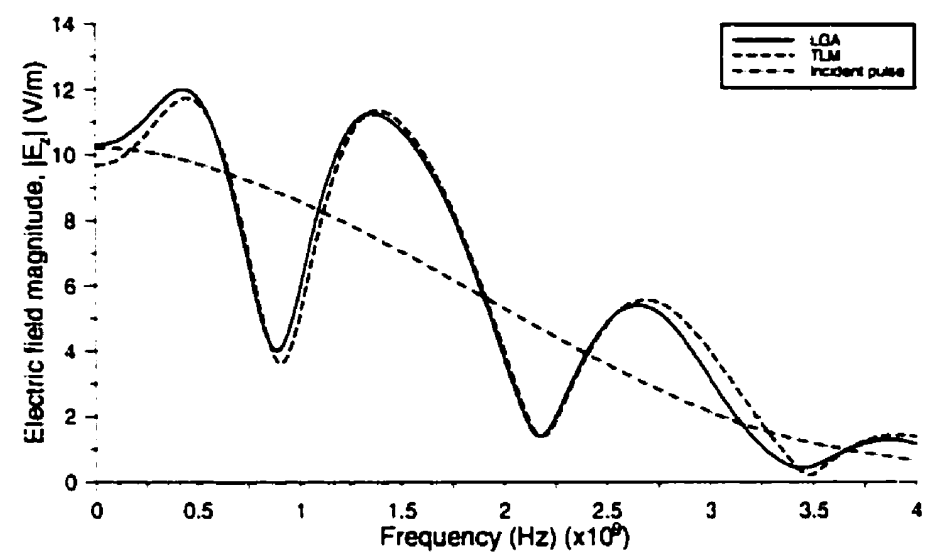

(b)

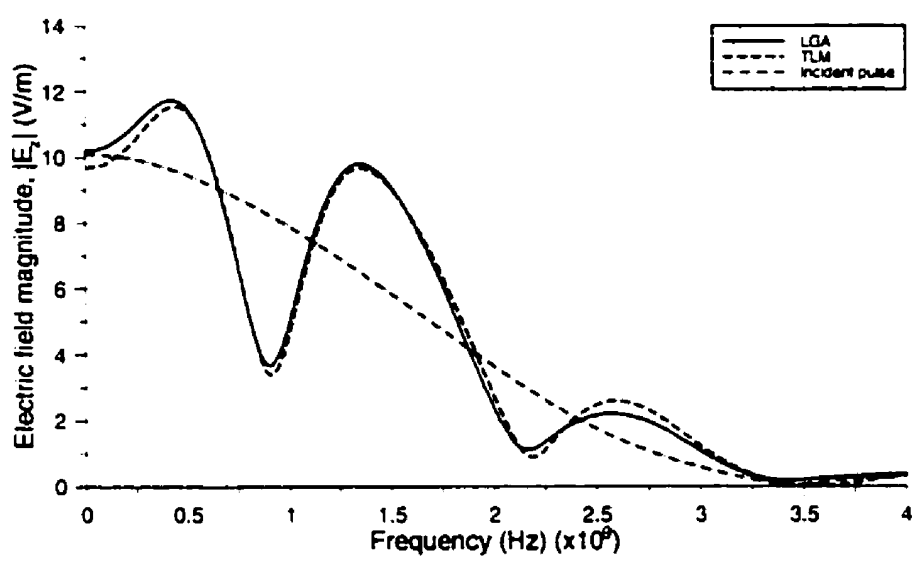

(c)

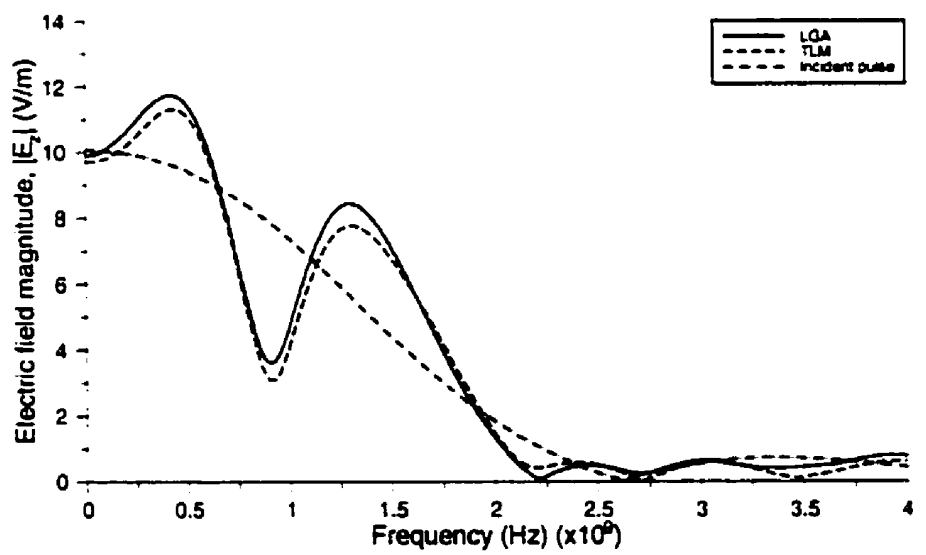

Figure 4.14: Frequency response of window 1 for $604 \times 1204$ cylinder crosssectional area computed with window dimensions of (a) $80 \Delta X 80 \Delta$, (b) $1204 \times 1204$, and (c) $1604 \times 1604$. 
(a)

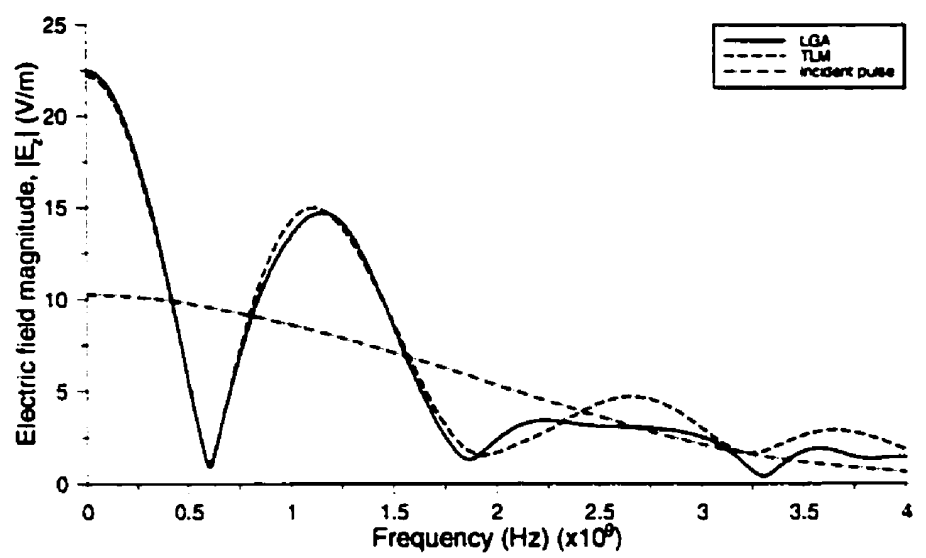

(b)

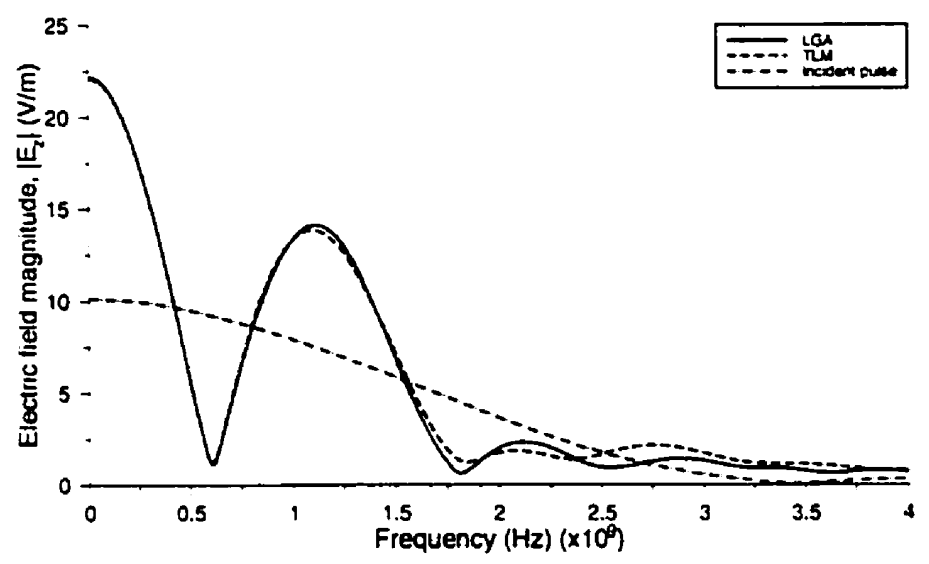

(c)

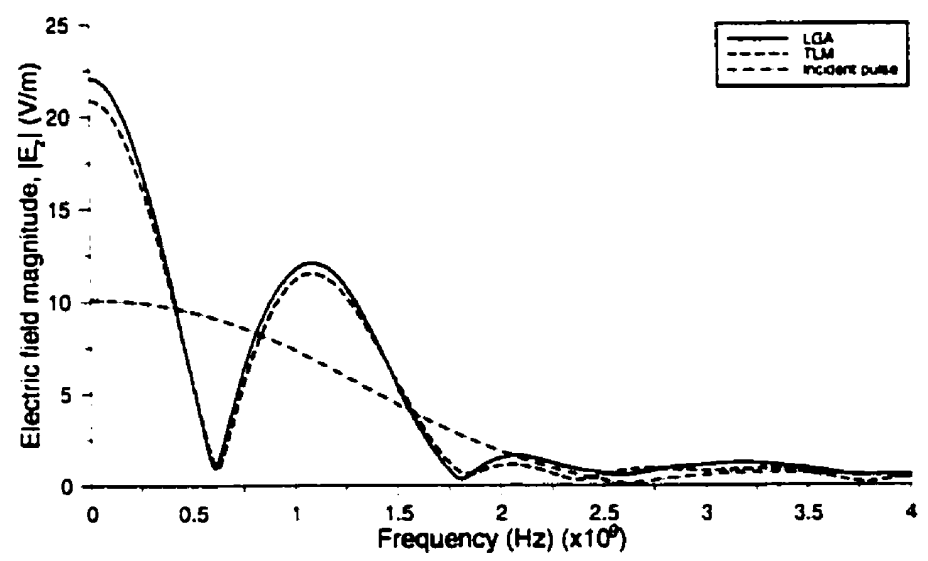

Figure 4.15: Frequency response of window 2 for $60 \Delta \times 120 \Delta$ cylinder crosssectional area computed with window dimensions of (a) $80 \Delta X 804$, (b) $120 \Delta \times 1204$, and (c) $160 \Delta \times 160 \Delta$. 
(a)

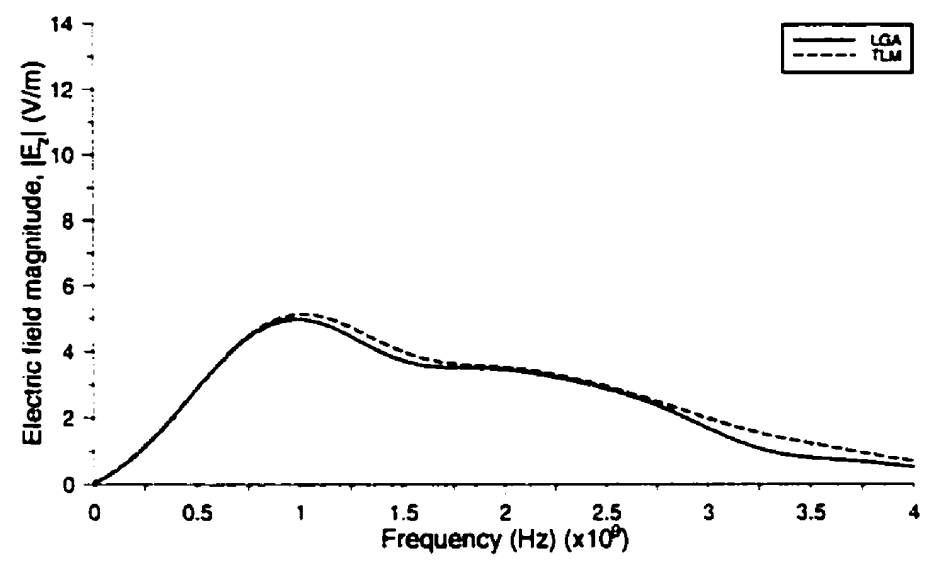

(b)

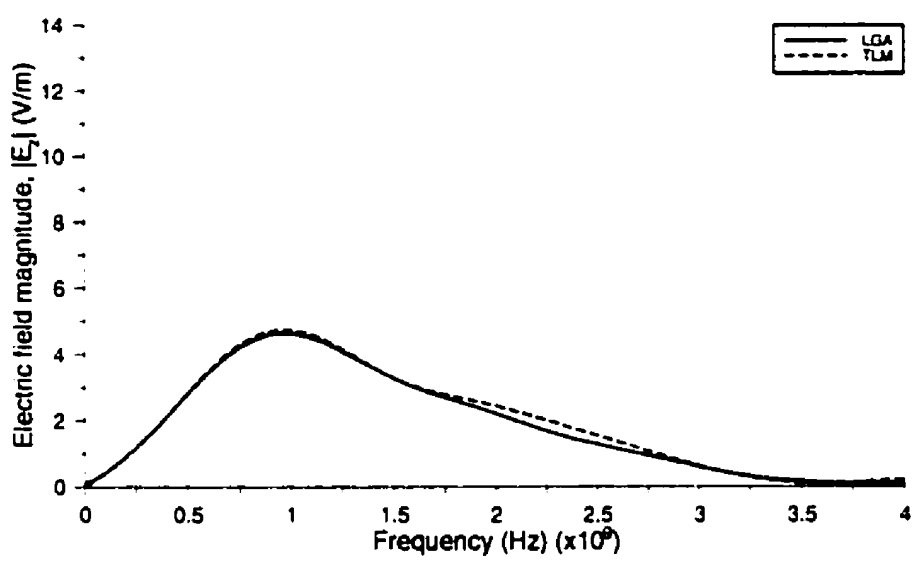

(c)

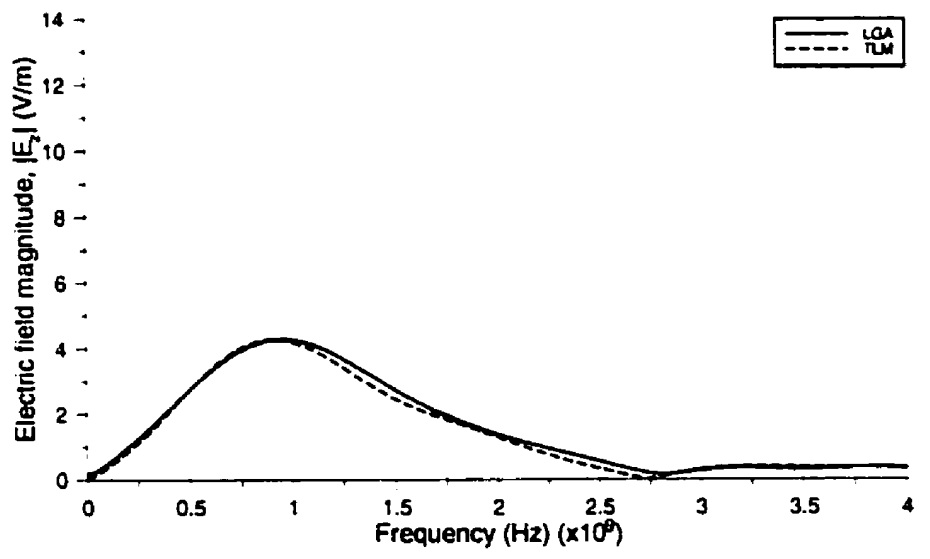

Figure 4.16: DFT of backward scatter response for $604 X 1204$ cylinder crosssectional area computed with window dimensions of $(a) 80 \Delta X 80 \Delta$. (b) $120 \Delta X 120 \Delta$, and (c) $160 \Delta X 160 \Delta$. 
(a)

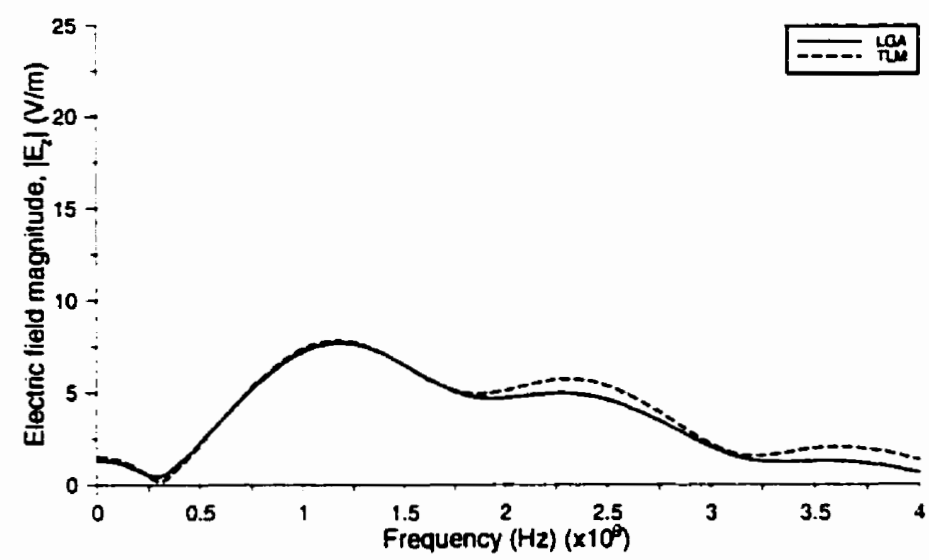

(b)

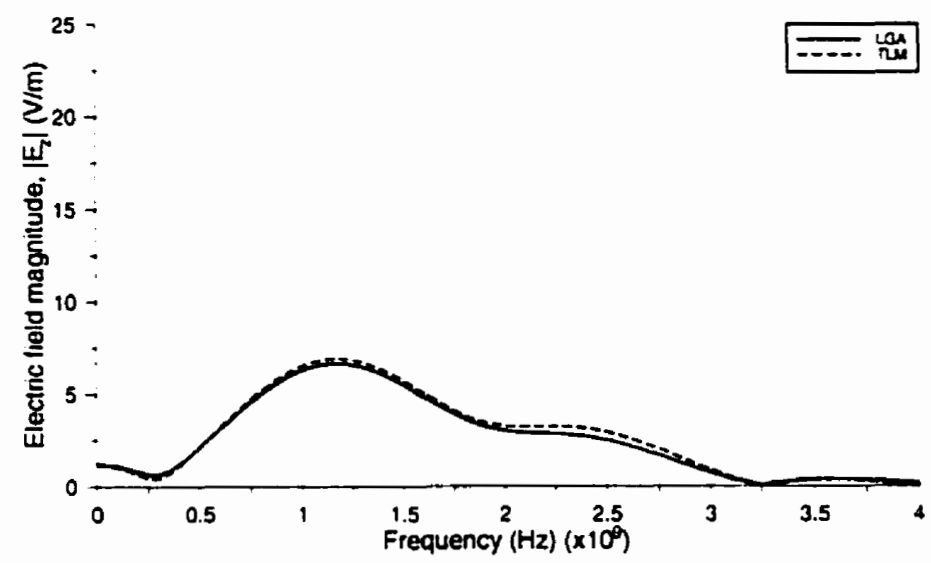

(c)

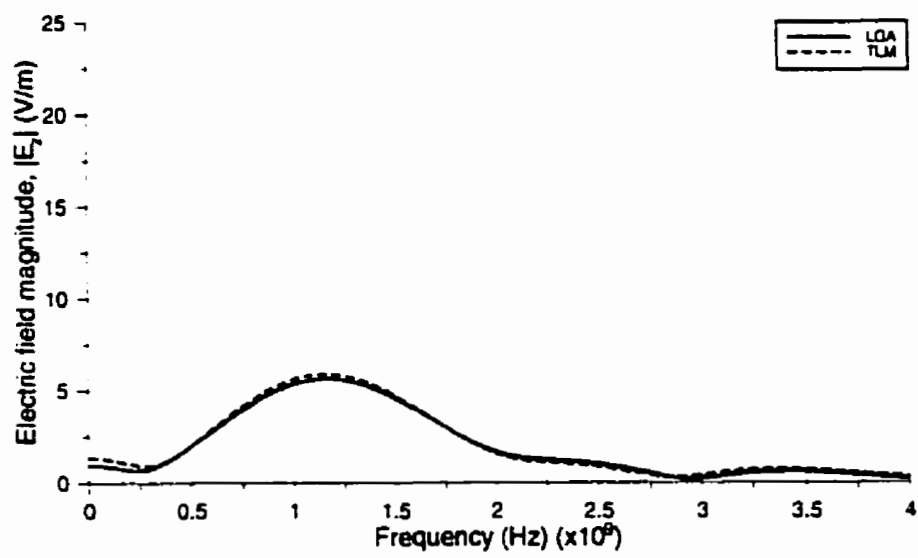

Figure 4.17: DFT of forward scatter response for $604 X 120 \Delta$ cylinder crosssectional area computed with window dimensions of (a) $804 X 804$, (b) $120 \Delta \times 120 \Delta$, and (c) $160 \Delta \times 1604$. 
The frequency responses to the input Gaussian for both sampling regions were computed by the frequency response of the incident pulse on the object, and the scattered output,

$$
\begin{aligned}
& \text { Forward_scat }(f)=\frac{E_{\text {fonward_scat }}(f)}{E_{\text {incident }}(f)} \\
& \text { Backward_scat }(f)=\frac{E_{\text {backward_scat }}(f)}{E_{\text {incident }}(f)}
\end{aligned}
$$

The resulting waveforms in the frequency domain are shown in Figures 4.18 and 4.19 for both the forward and backward scattered outputs. The LGA response to the incident Gaussian pulse in both cases is almost identical to the TLM computation. 
(a)

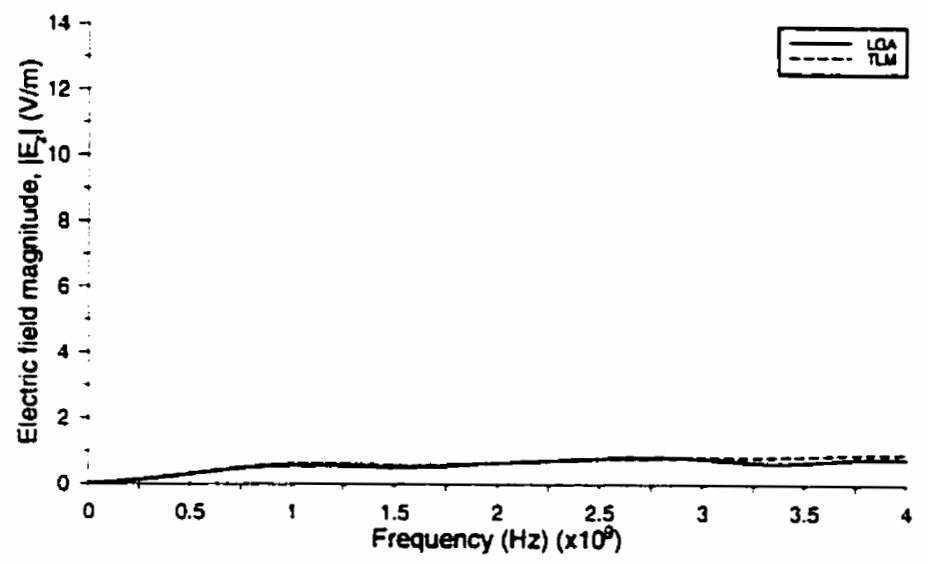

(b)

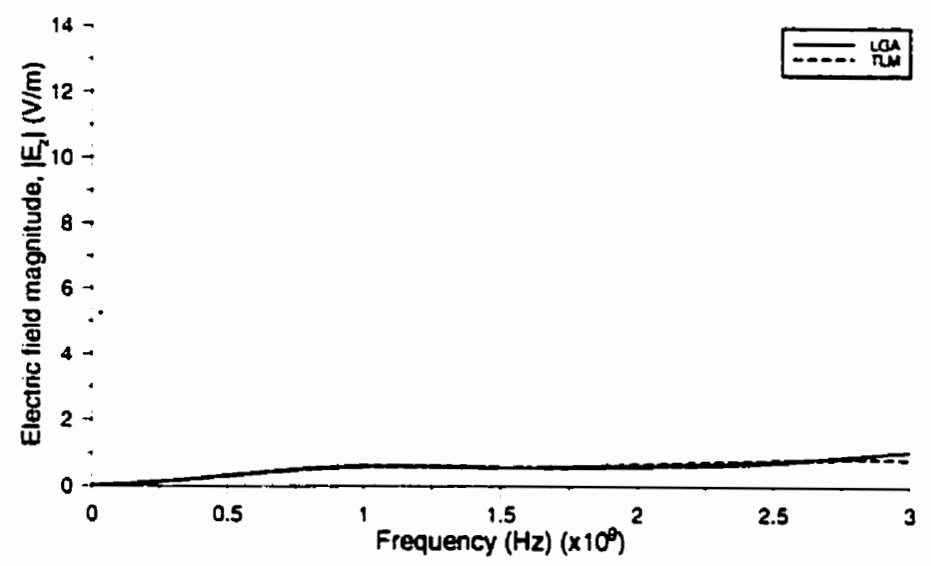

(c)

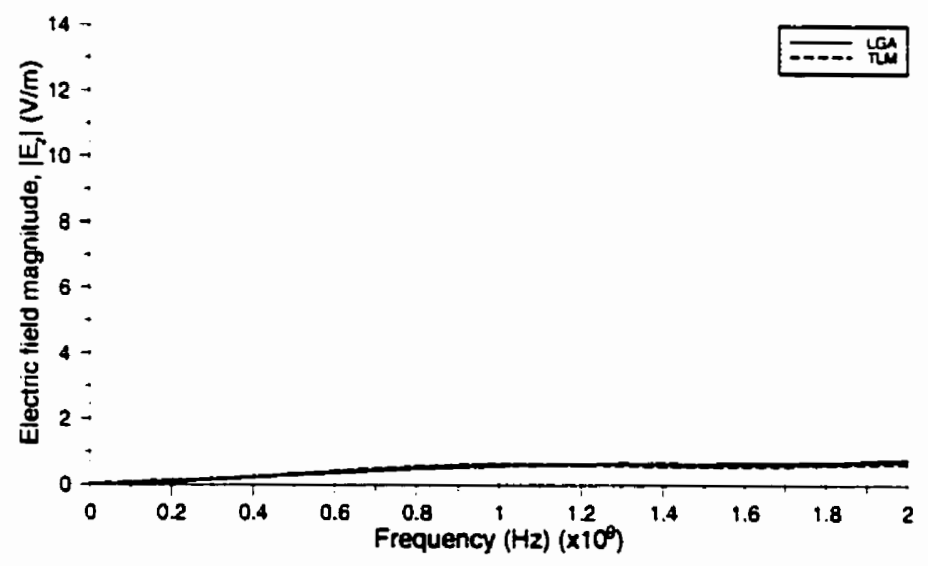

Figure 4.18: Response of the backward scatter to the incident pulse for $60 \Delta X$ $120 \Delta$ cylinder cross-sectional area with window dimensions of (a) $80 \Delta X 80 \Delta$, (b) $120 \Delta X 120 \Delta$, and (c) $160 \Delta X 160 \Delta$. 
(a)

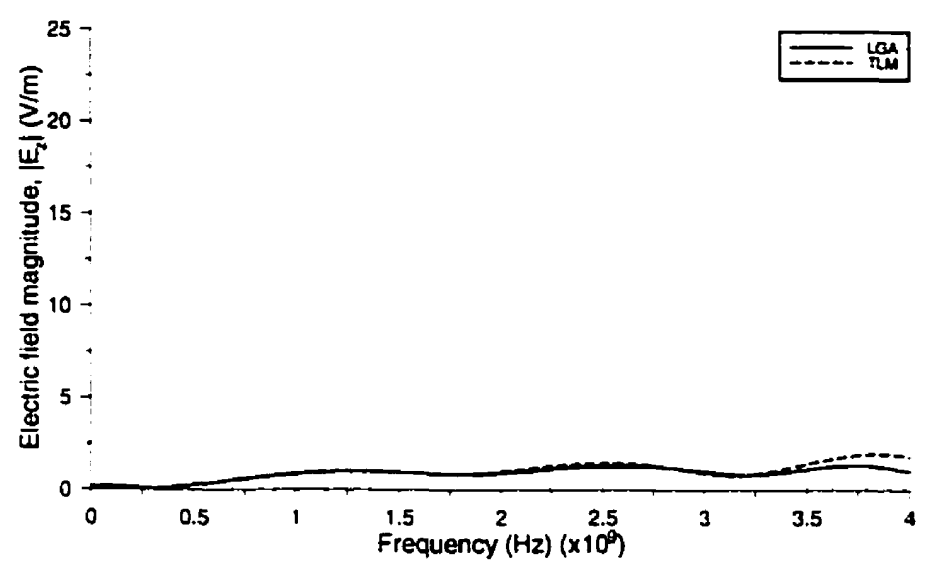

(b)

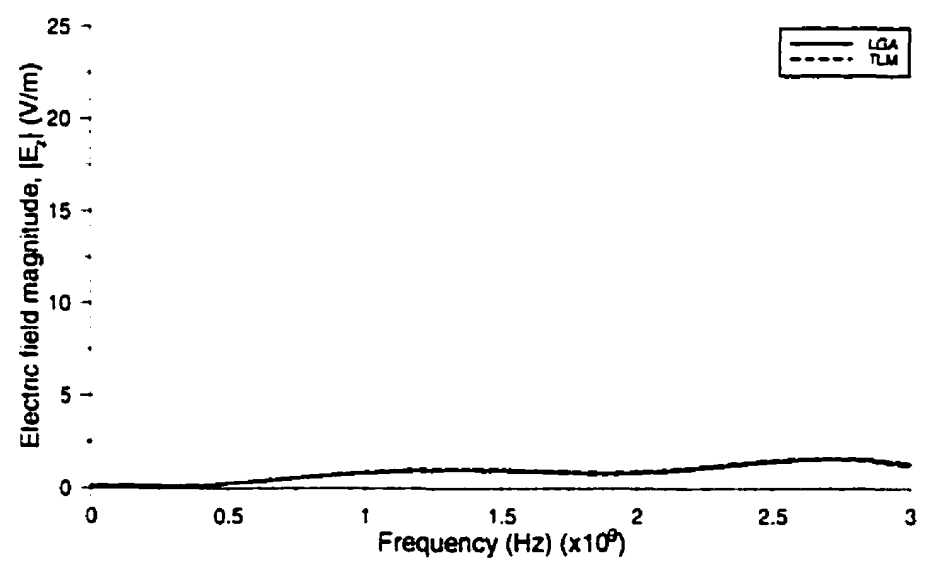

(c)

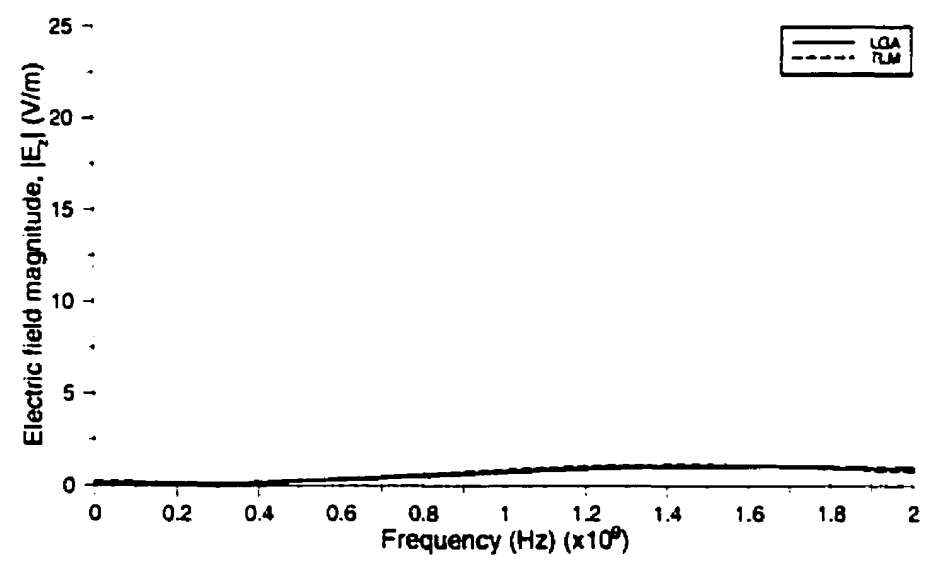

Figure 4.19: Response of the forward scatter to the incident pulse for $60 \Delta X 120 \Delta$ cylinder cross-sectional area with window dimensions of (a) $80 \Delta X$ $80 \Delta$, (b) $120 \Delta X 1204$, and (c) $160 \Delta X 160 \Delta$. 


\subsubsection{Effect of Cylinder Dimensions on Scattered Signals}

Three cases were studied involving varying sizes of the cylinder cross-sectional area, shown in Figure 4.20, and are listed below:

$$
\begin{aligned}
& \text { Case 1: area }=60 \Delta_{L G . t} \times 120 \Delta_{L G A} \\
& \text { Case 2: area }=140 \Delta_{L G .} \times 120 \Delta_{L G A} \\
& \text { Case 3: area }=60 \Delta_{L G . t} \times 240 \Delta_{L G . A}
\end{aligned}
$$

Each case was simulated with sampling window sizes of $120 \Delta_{L G . t} \times 120 \Delta_{L G . t}$ in the LGA mesh and a window diameter of $141 \Delta_{T L M}$ in the TLM mesh.

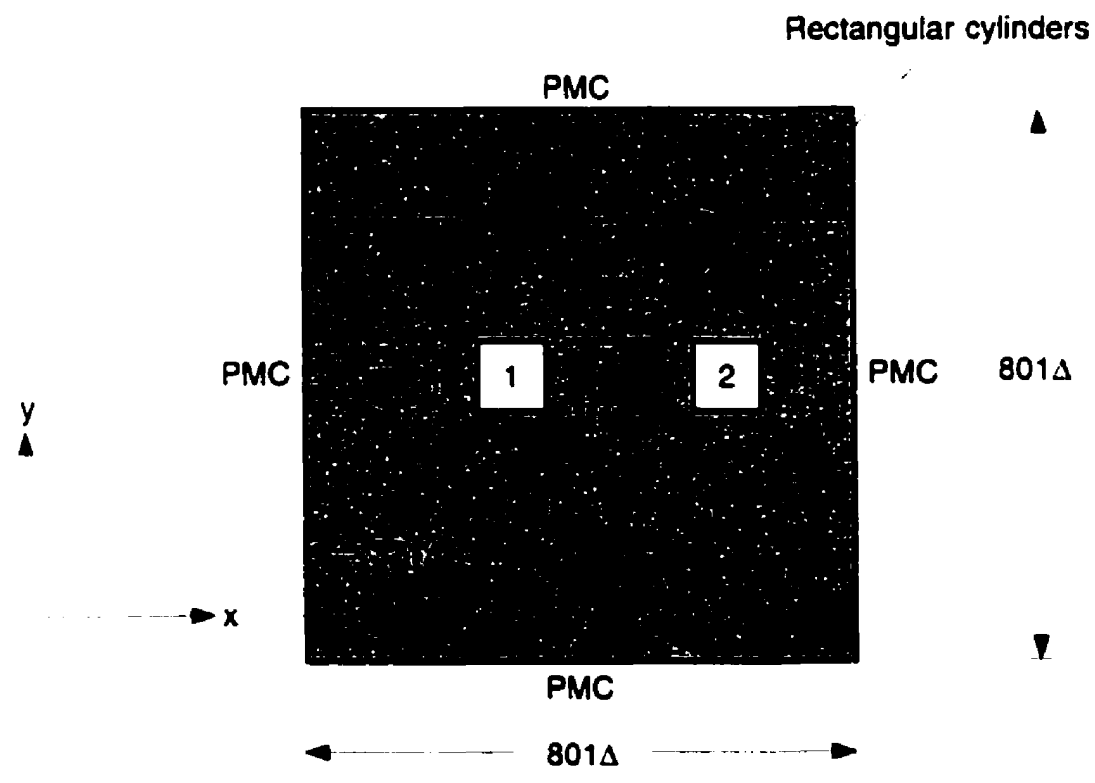

Figure 4.20: Lattice configuration with rectangular cylinders of varying crosssectional areas. 


\subsubsection{Time Domain}

The time domain traces for each scattering case are given in Figures 4.21 and 4.22, for sampling windows 1 and 2, respectively. Figures 4.21 (a) and 4.22 (a) represent the electric field for the case with no scattering object. For the field computed by window l, only the incident Gaussian at $t=440 \Delta t$ is common in both the non-cylinder and cylinder waveforms. The $60 \Delta \times 240 \Delta$ case, shown in Figure 4.21 (d), has an effect similar to that of a full PMC boundary. The second pulse in Figure 4.21 (d) represents that part of the wave that was reflected by the cylinder. The wave meets the cylinder after approximately 680 time steps and is bounced-back in the negative $x$-direction of the mesh returning towards the first window. The peak is observed at $t=857 \Delta t$. Had it struck a PMC wall, the pulse would have been computed at approximately $t=906 \Delta t$.

The smaller object with $60 \Delta \times 120 \Delta$ cross-section in Figure 4.21 (b) produces a similar waveform but with a substantially reduced reflected pulse, indicative of the decreased length of the cylinder cross-section in this case. Less particles are reflected by the object thus a lower pulse level occurs. The time at which the pulse peak is observed, at $t=845 \Delta t$, is slightly less than that for the larger $60 \Delta \times 240 \Delta$ object case.

In the $140 \Delta \times 120 \Delta$ object case in Figure 4.21 (c), the wave meets the cylinder after approximately 567 time steps before a portion of it is reflected back towards the first window. The width of this object is just over twice that of the $60 \Delta \times 120 \Delta$ object case, but their lengths are identical. From the trace in Figure 4.21 (c), it would seem that the arrival of the reflected wave at window 1 occurs with the departure of the tail end of the incident pulse.

The waveforms shown in Figure 4.22 for each scattering case are relatively similar. A slight decline in the field level for the $60 \Delta \times 120 \Delta$ and $140 \Delta \times 120 \Delta$ is noted in comparison to the no object simulation. In addition the $60 \Delta \times 240 \Delta$ case in Figure 4.22 (d), has a lower pulse amplitudes in comparison to Figures 4.22 (b) and (c). 


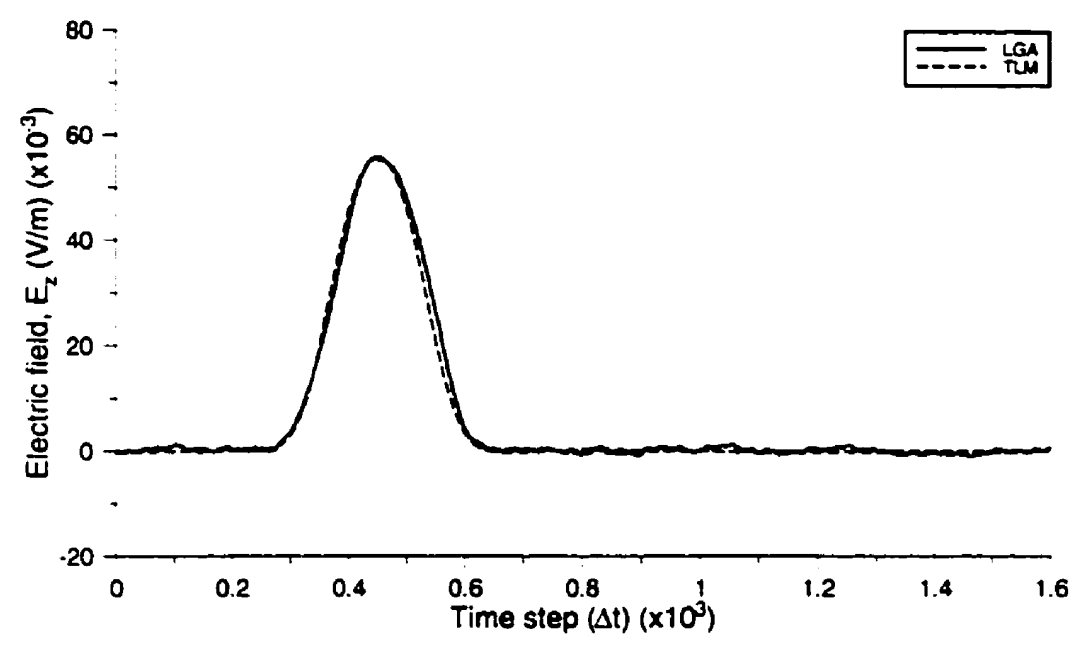

(a) no object

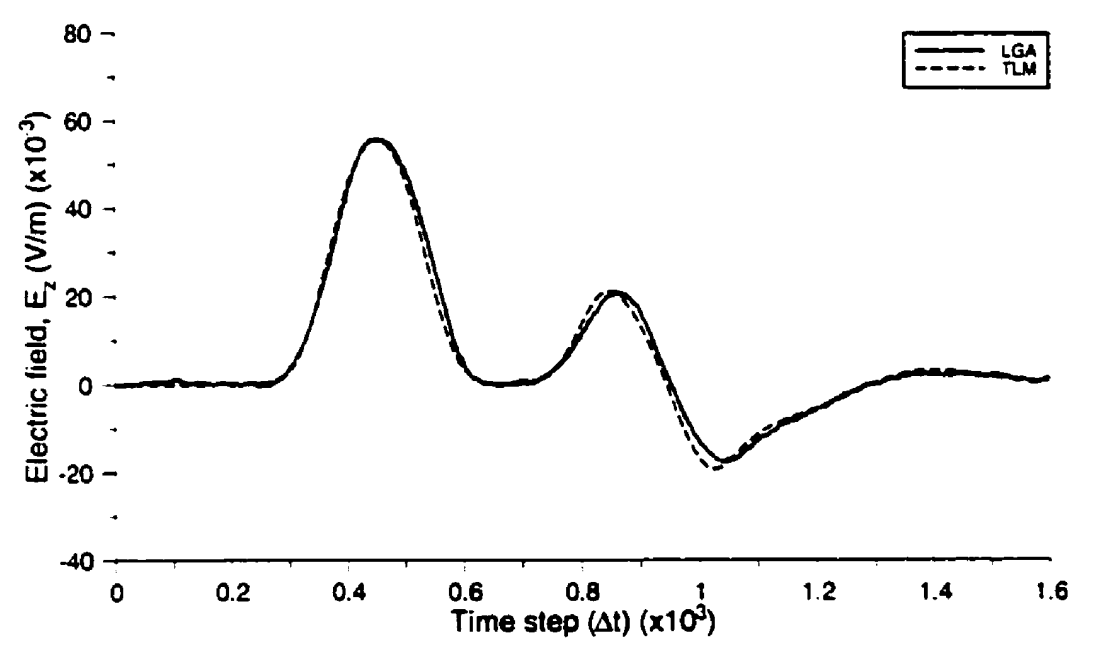

(b) $60 \Delta \times 120 \Delta$ cylinder cross-section

Figure 4.21: Total field (incident and backward scatter) waveforms of window 1 for (a) no object, and for a cylinder cross-sectional area of (b) $60 \Delta$ $X 1204$, (c) $140 \Delta X 1204$, and (d) $60 \Delta X 240 \Delta$. 


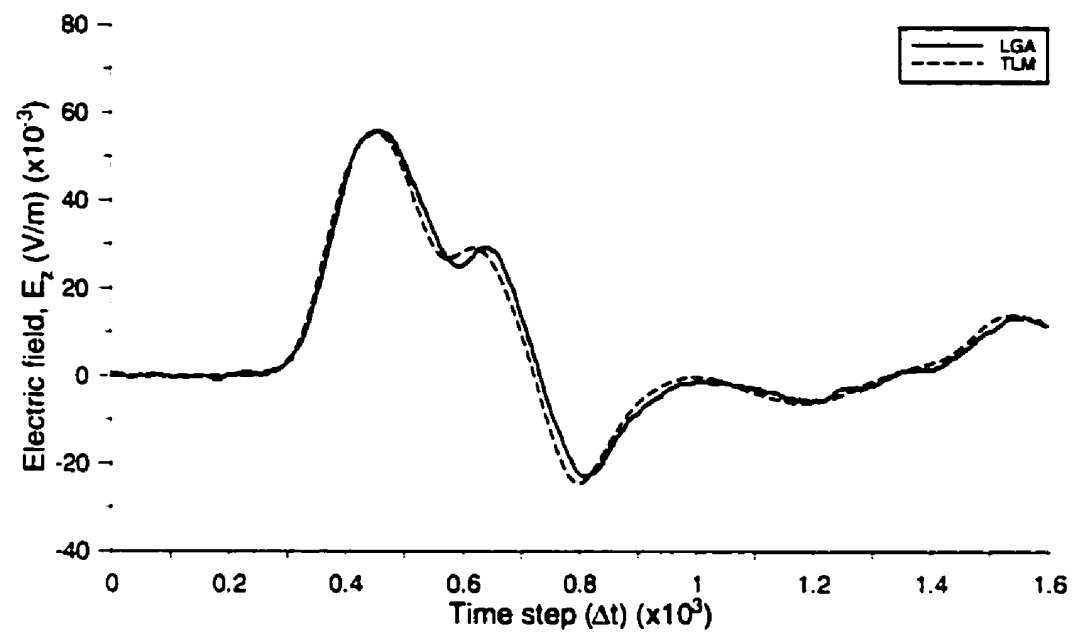

(c) $140 \Delta \times 120 \Delta$ cylinder cross-section

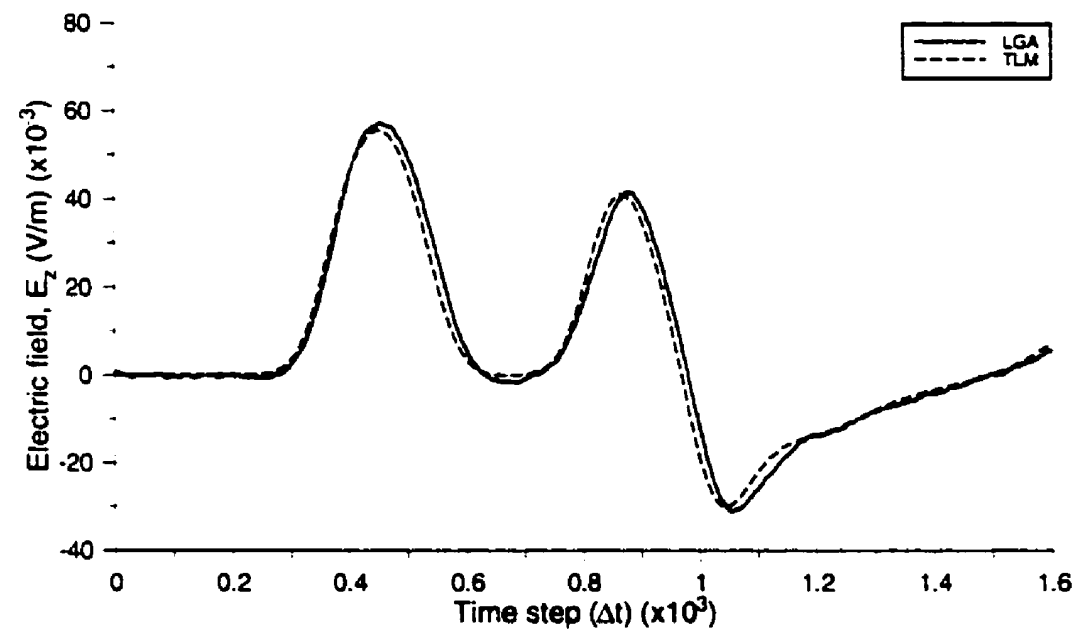

(d) $60 \Delta \times 240 \Delta$ cylinder cross-section

Figure 4.21 (continued) 


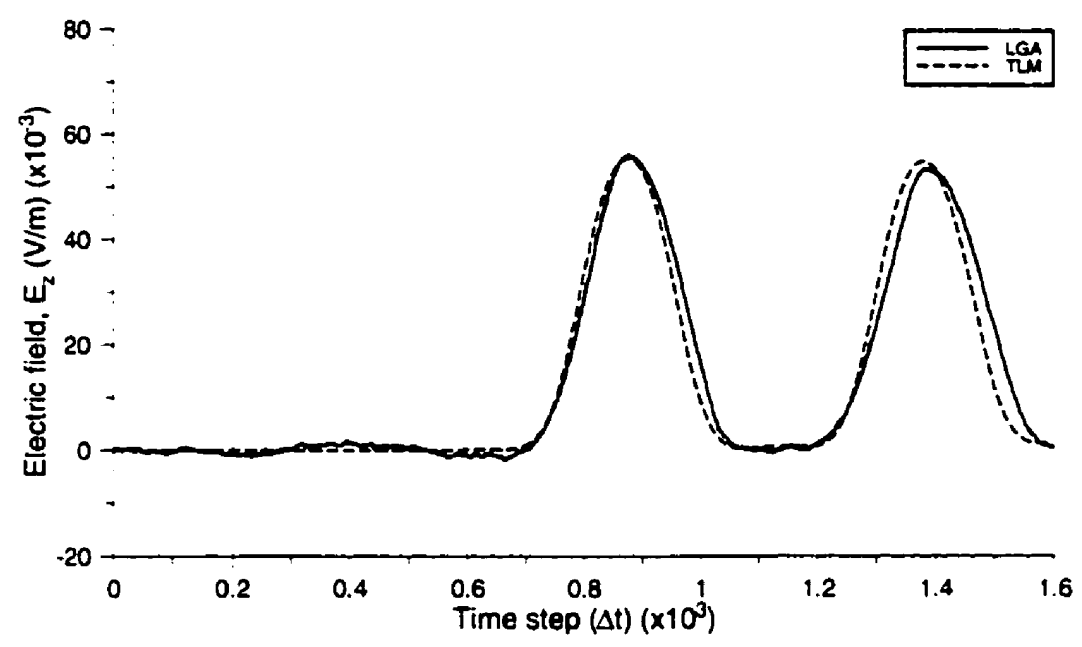

(a) no cylinder

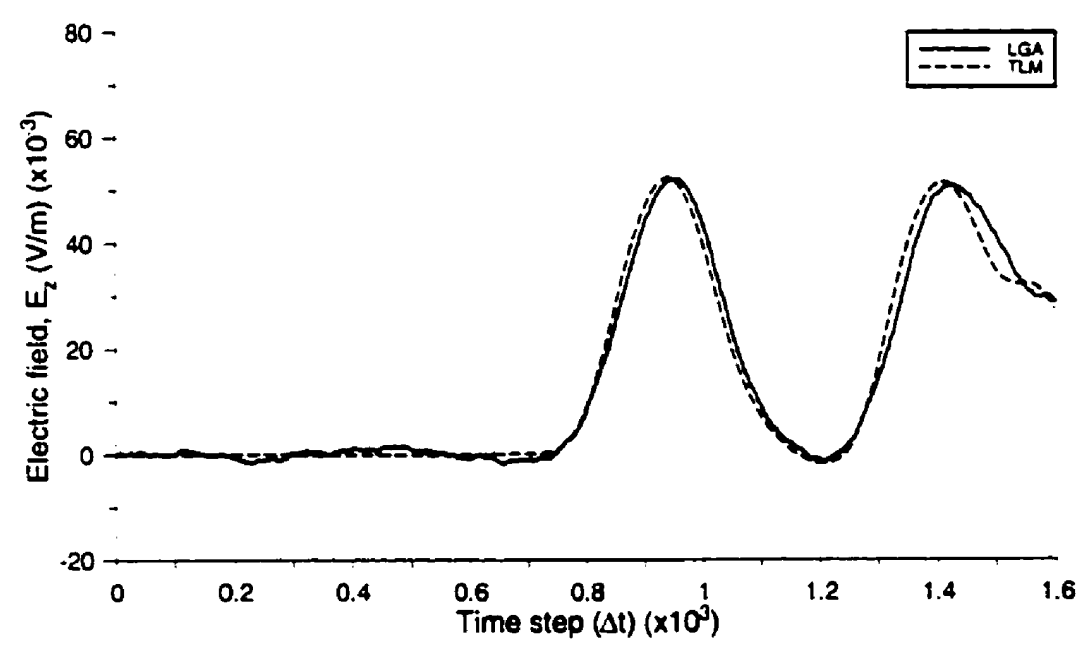

(b) $60 \Delta \times 120 \Delta$ cylinder cross-section

Figure 4.22: Total field (incident and forward scatter) waveforms of window 2 for (a) no object, and for a cylinder cross-sectional area of (b) $60 \Delta \mathrm{X}$ 1204, (c) $140 \Delta X 1204$, and (d) $60 \Delta \times 240 \Delta$. 


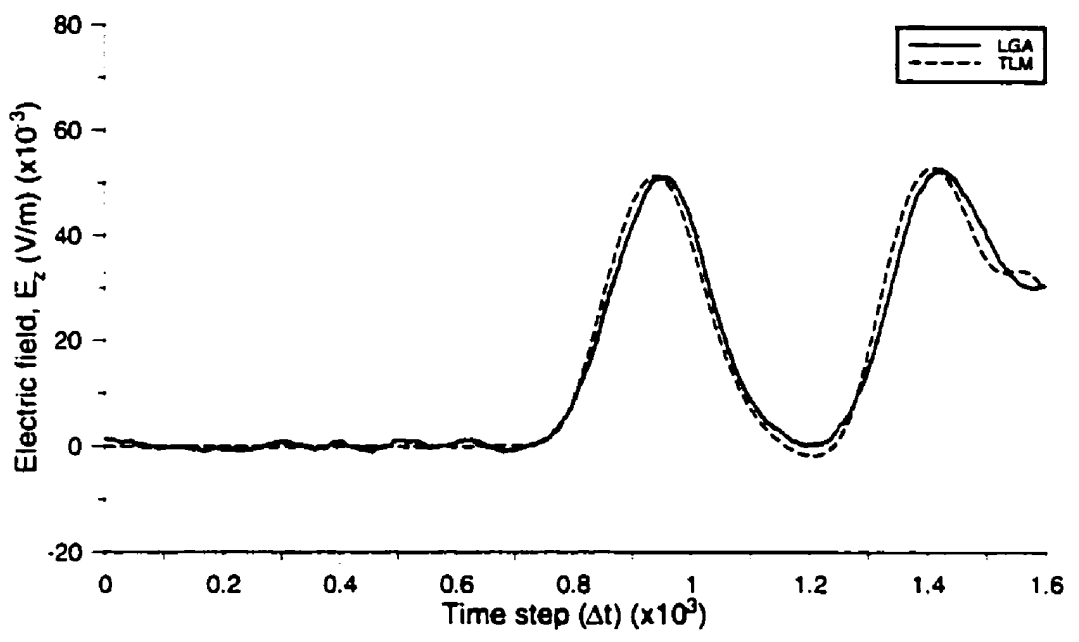

(c) $140 \Delta \times 120 \Delta$ cylinder cross-section

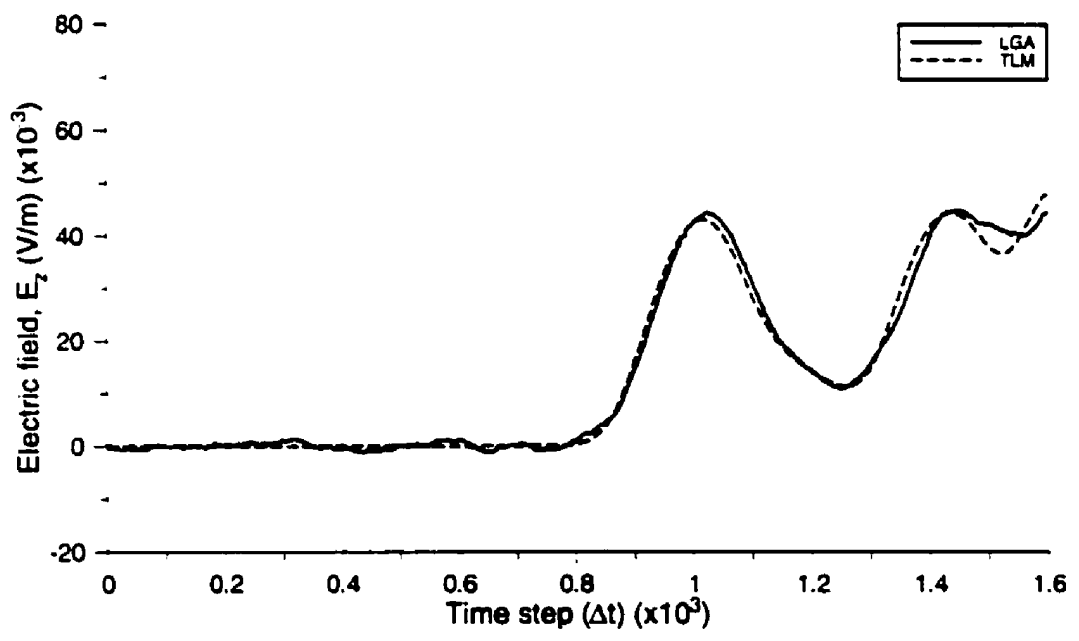

(d) $60 \Delta \times 240 \Delta$ cylinder cross-section

Figure 4.22 (continued) 
The time domain forward and backward scatter waveforms were obtained by subtracting the waveform computed by the no object simulation from each of the three cases. The resulting waveforms are shown in Figures 4.23 and 4.24 for the backward and forward scatter, corresponding to windows 1 and 2, respectively. The waveforms of the forward scatter in Figure 4.24, with respect to the timing of the peaks and nulls, are similar for all three cases. It should be noted that the location (i.e. $x$-coordinate) of the rightmost wall of the cylinder was kept constant for all three cases. Thus, the approximate timing of the nulls and peaks of the forward scatter are similar for all three cases. However, while the magnitudes of the pulses and nulls are similar for the $60 \Delta \times 120 \Delta$ and $140 \Delta \times 120 \Delta$ cases, those computed for the $60 \Delta \times 240 \Delta$ cylinder are greater.

Differences occur in the time of the appearance of the peaks and nulls for the backward scatter waveforms of Figure 4.23. These are similar for the $60 \Delta \times 240 \Delta$ and $60 \Delta$ X $120 \Delta$ cases, but are observed slightly earlier in the $140 \Delta \times 120 \Delta$ case due to its larger width. The effect of the $140 \Delta \times 120 \Delta$ cylinder cross-section on the waveform is more clearly illustrated in Figure 4.23 (b), in comparison to the trace shown in Figure 4.21 representing the incident and backward scatter field.

Windows 1 and 2, in Figures 4.23 and 4.24, show only the backward and forward scattering resulting from the object. However, to obtain a full assessment of the scattering behaviour due to each cylinder, it would be necessary to place several windows around the objects to observe the scattering pattern over the entire space. The objective of this chapter, however, was to compare the TLM and LGA simulations. The traces shown in Figures 4.21 to 4.24 obtained by the two methods are similar, and illustrate general agreement in the computed results. Thus, a complete assessment was not performed, as the scattering results are also well known [39]. 
(a)

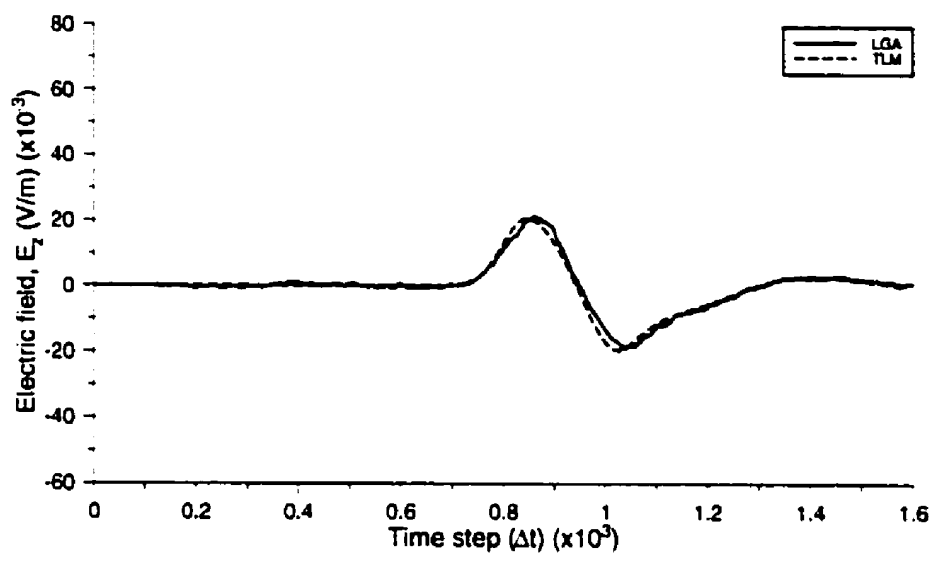

(b)

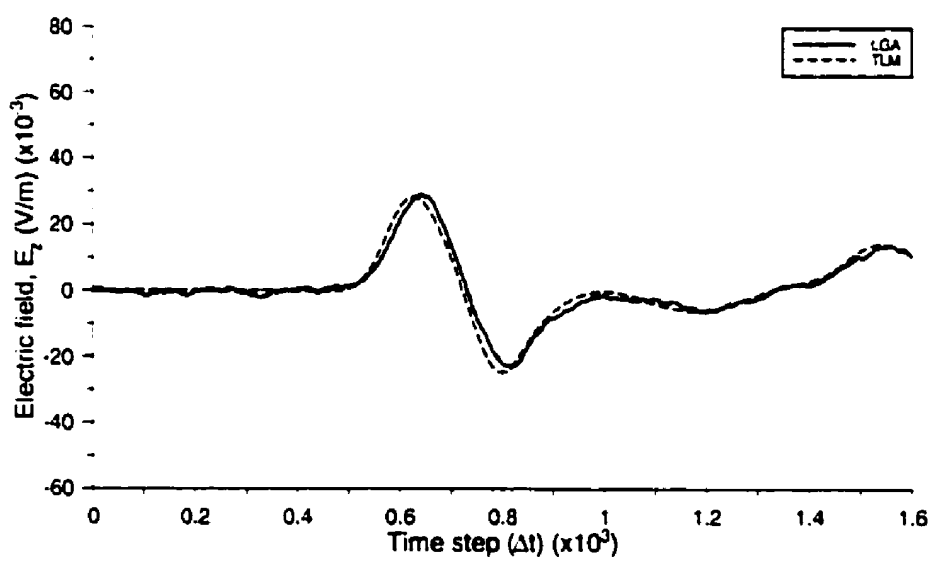

(c)

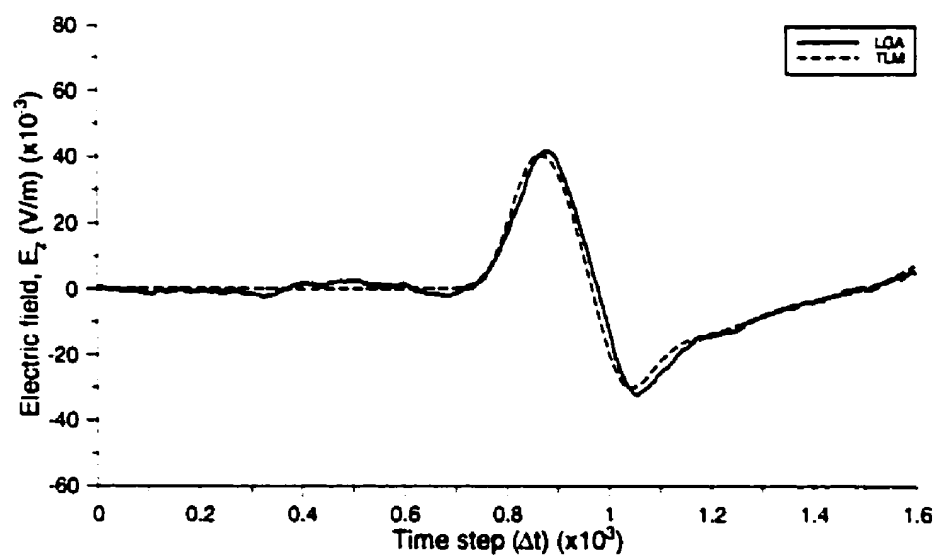

Figure 4.23: Time domain waveforms of backward scatter in window I for cylinder cross-sectional area of (a) $60 \Delta X 1204$, (b) $140 \Delta X 1204$, and (c) $604 \times 2404$. 
(a)

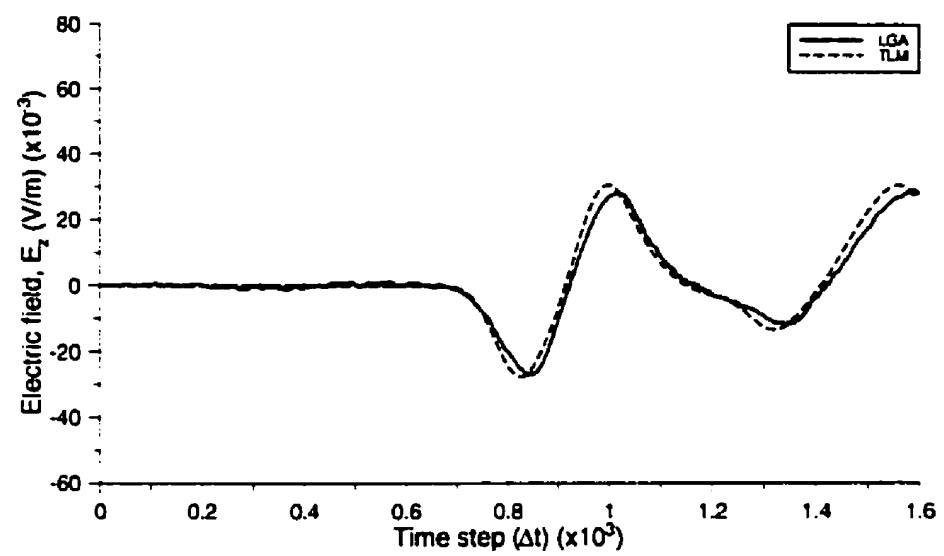

(b)

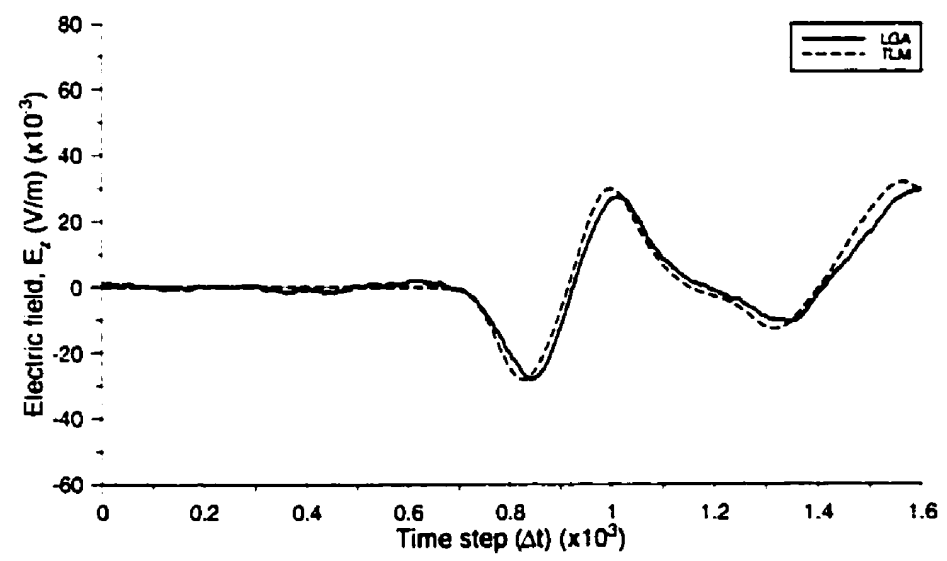

(c)

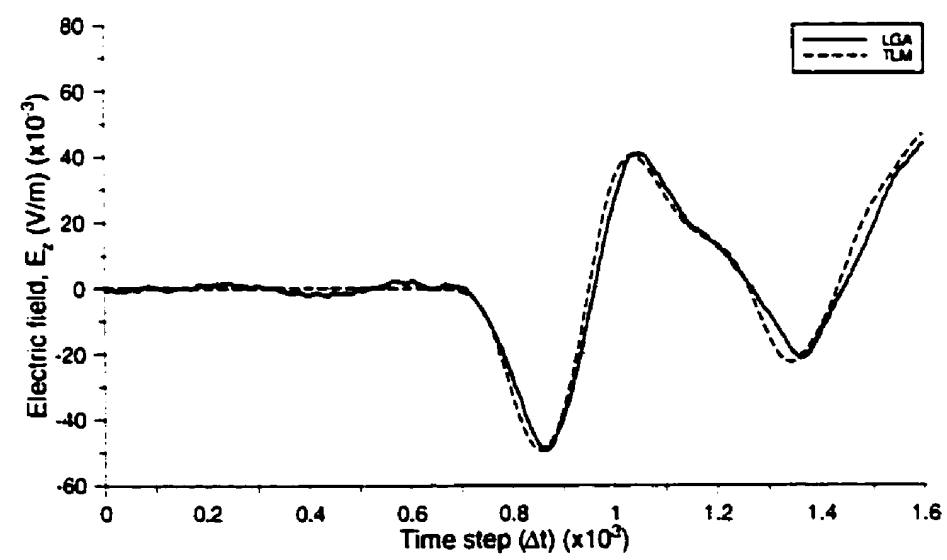

Figure 4.24: Time domain waveforms of forward scatter in window 2 for cylinder cross-sectional area of (a) $60 \Delta X 1204$, (b) $140 \Delta X 1204$, and (c) $60 \Delta \times 240 \Delta$. 


\subsubsection{Frequency Domain}

The frequency response of the time domain simulations shown in Figures 4.21 (incident pulse and backward scatter) and 4.22 (incident and forward scatter) are given in Figures 4.25 and 4.26 for windows 1 and 2, respectively. Analogous to the trends discussed for the time domain waveforms, the responses in window $\mathrm{l}$ are more similar for the $60 \Delta \mathrm{X}$ $120 \Delta$ and $60 \Delta \times 240 \Delta$ cross-section simulations. Resonant peaks occur at approximately the same frequencies in these two cases, but the peak magnitudes are greater for the $60 \Delta \mathrm{X}$ $240 \Delta$ case in Figure 4.25 (c). The null at approximately $0.8 \mathrm{GHz}$ also has a lower magnitude than that for the $60 \Delta \times 120 \Delta$ response in Figure 4.25 (a). The response for the $140 \Delta \times 120 \Delta$ cross-section in Figure 4.25 (b) is quite different than that computed for the other two cylinders. However, a common null occurs around $0.8 \mathrm{GHz}$, but is not as deep and does not create two distinct beams as for the other two cylinder geometries. The greatest resonance occurs at approximately $0.6 \mathrm{GHz}$ at around the same frequency as the first resonance for the other two cases.

The responses for window $\mathbf{2}$ in Figure $\mathbf{4} .26$ have a common pattern for all three cases. This is to be expected, as the corresponding time domain waveforms in Figure 4.22 were similar. The peaks and nulls are of similar magnitudes, but the second peak shown for the $60 \Delta \times 240 \Delta$ is slightly less than that for the other two cases. The magnitudes of the resonant peaks are much greater than the field levels computed in window 1.

The frequency responses resulting from the backward and forward scattering are given in Figures 4.27 and 4.28, respectively. The $60 \Delta \times 240 \Delta$ and $60 \Delta \times 120 \Delta$ responses in Figures 4.27 (a) and (c) are somewhat alike in shape, but differ significantly in the

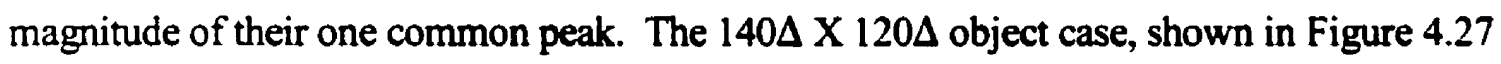
(b), consists of several nulls and peaks, caused by the interaction of scattered waves at the front and back surfaces of the thicker object. The waveforms of the forward scattering at window 2 are similar in each case, again a direct result of their comparable time domain traces in Figure 4.24. 
(a)

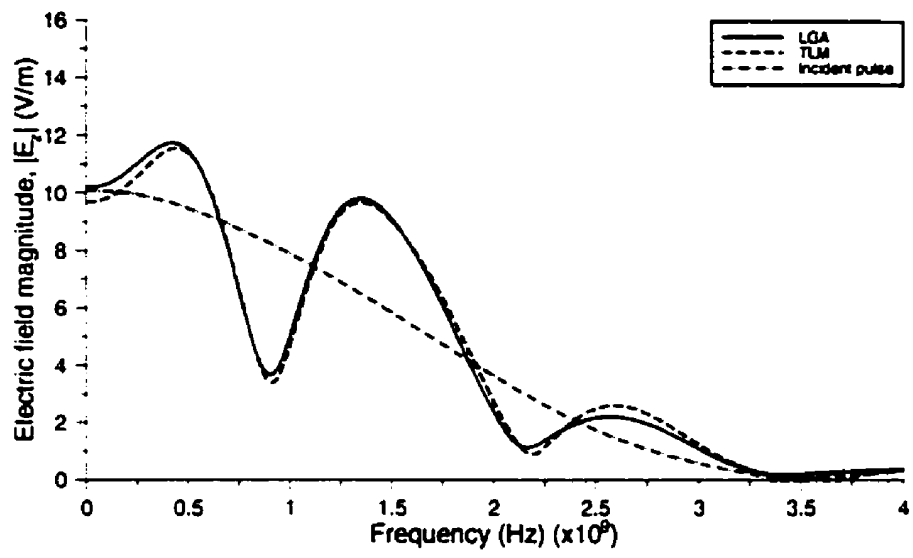

(b)

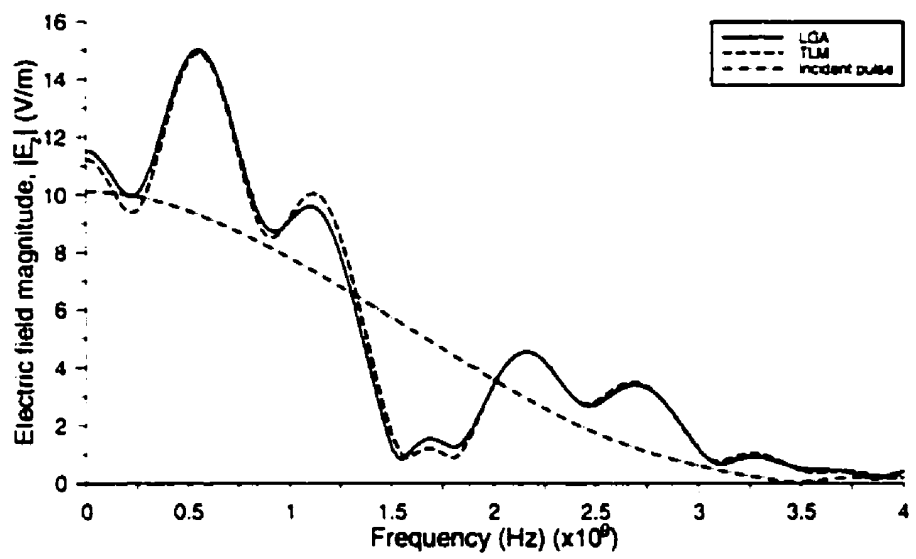

(c)

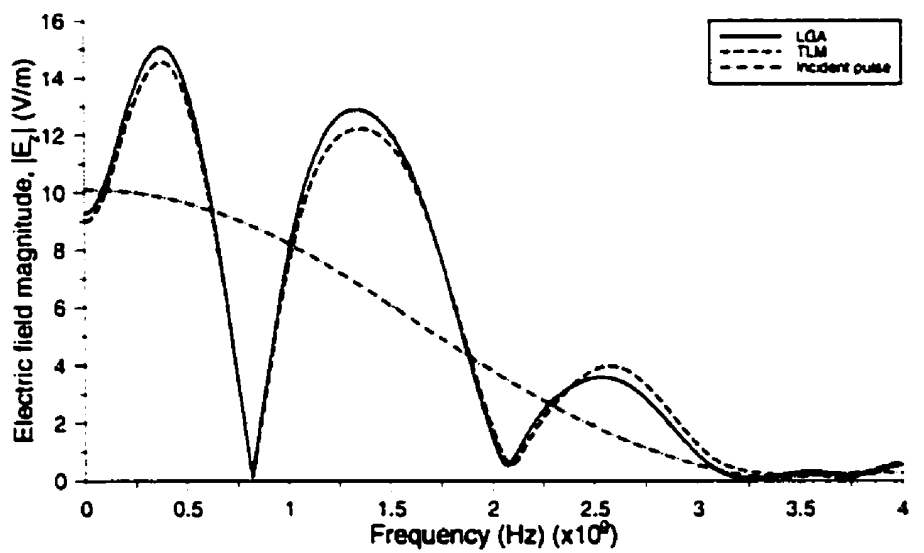

Figure 4.25: Frequency response (incident and backward scatter) in window 1 for a cylinder cross-sectional area of (a) $60 \Delta X 1204$, (b) $140 \Delta X 1204$, and (c) $604 \times 2404$. 
(a)

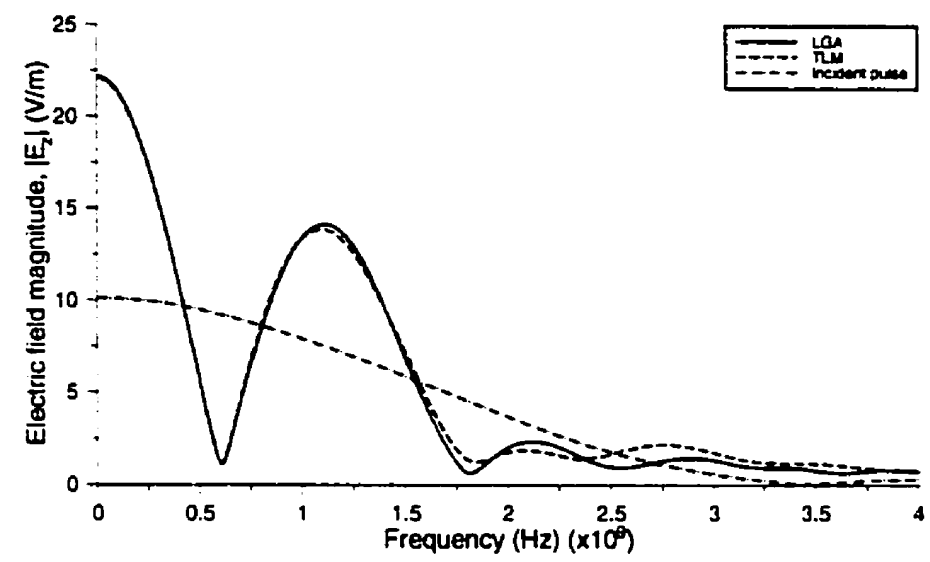

(b)

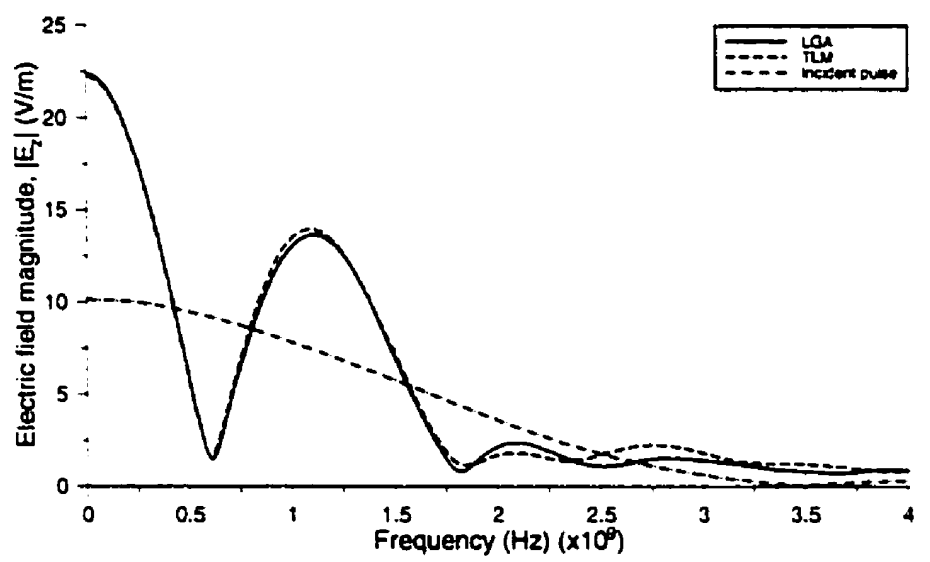

(c)

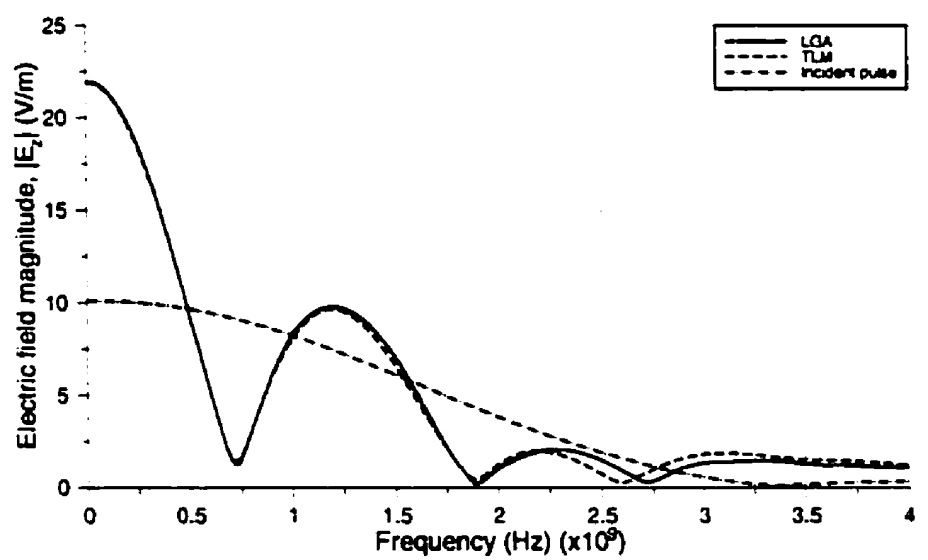

Figure 4.26: Frequency response (incident and forward scatter) of window 2 for a cylinder cross-sectional area of (a) $60 \Delta X 120 \Delta$, (b) $140 \Delta X 1204$. and (c) $60 \Delta X 240 \Delta$. 
(a)

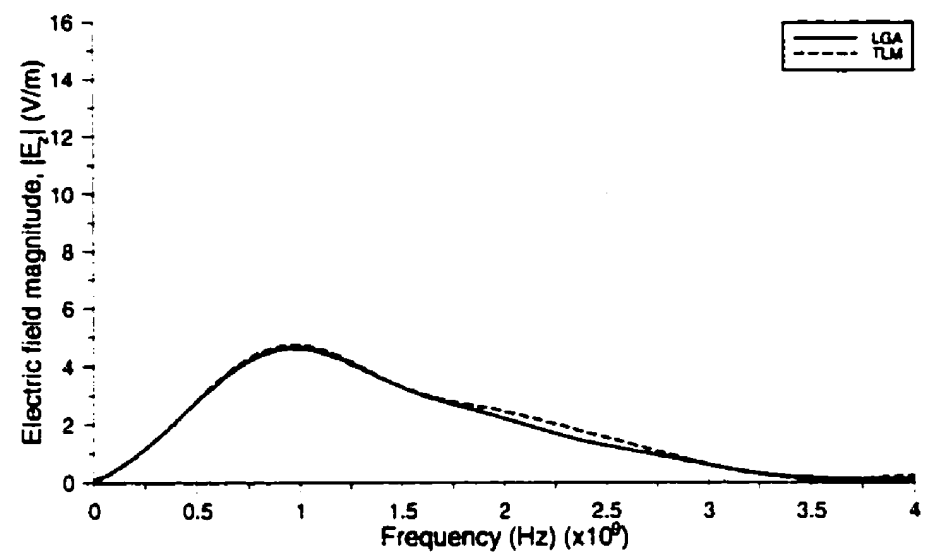

(b)

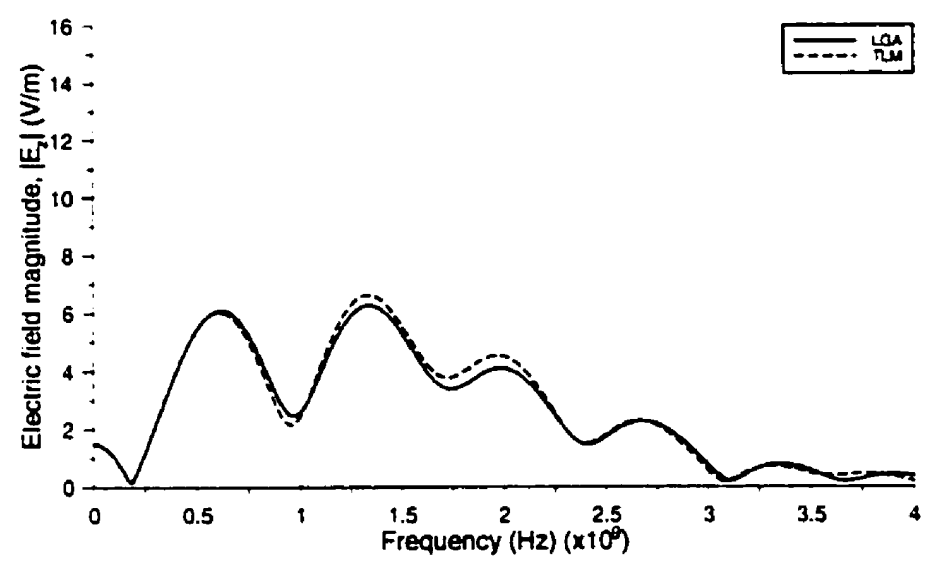

(c)

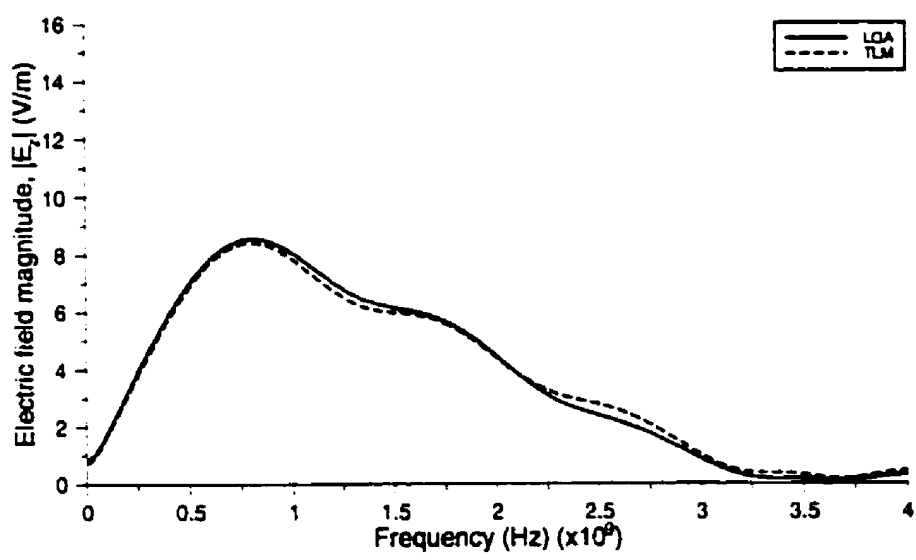

Figure 4.27: Frequency response of backward scatter in window 1 for a cylinder cross-sectional area of (a) $60 \Delta \times 120 \Delta$, (b) $140 \Delta X 1204$, and (c) $60 \Delta \times 240 \Delta$. 
(a)

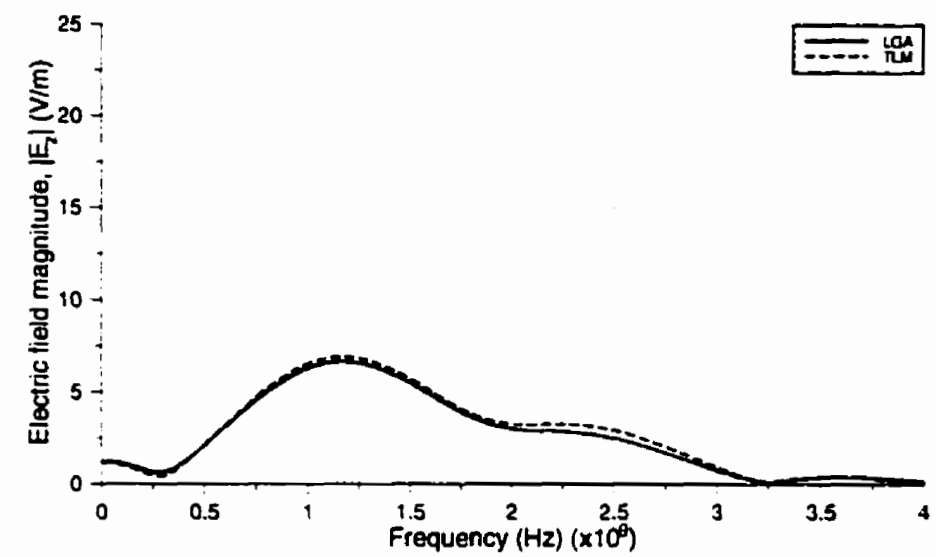

(b)

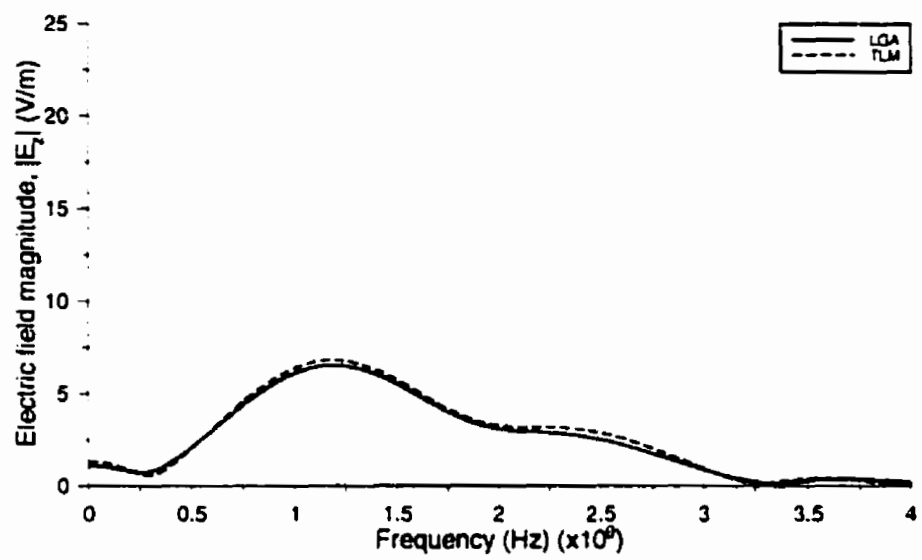

(c)

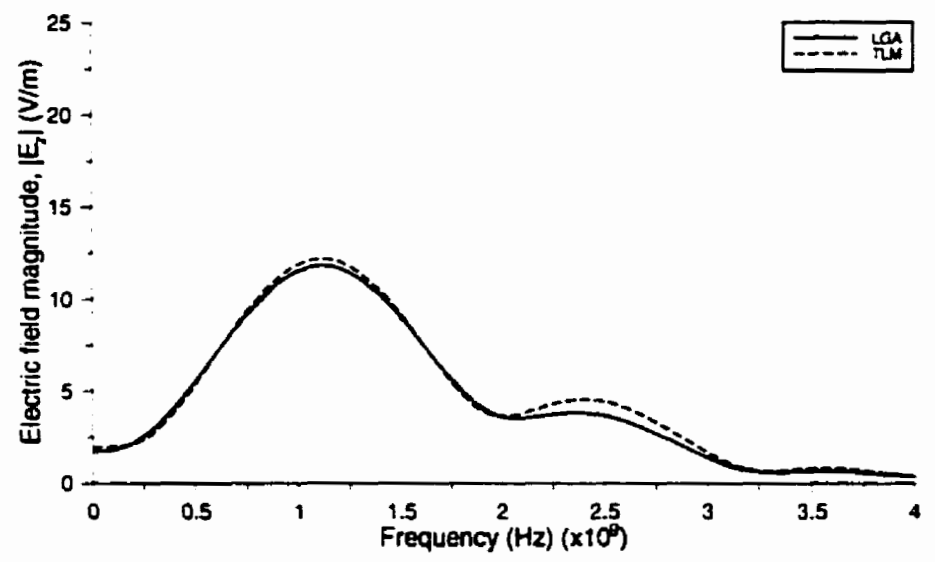

Figure 4.28: Frequency response of forward scatter in window 2 for a cylinder cross-sectional area of (a) $60 \Delta X 120 \Delta$. (b) $140 \Delta X 120 \Delta$, and (c) $60 \Delta X 240 \Delta$. 
The responses of the forward and backward scattering to the incident pulse in the mesh were computed using equations (4.29) and (4.30), and are shown in Figures 4.29 and 4.30. As expected, the responses corresponding to window 2 are comparable. TLM and LGA traces in both Figures 4.29 and 4.30 are almost identical. 
(a)

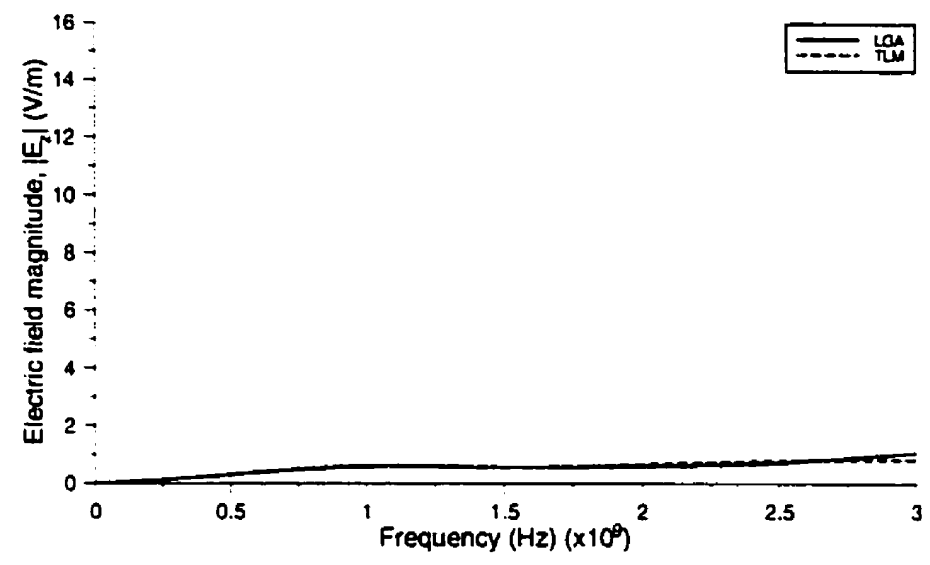

(b)

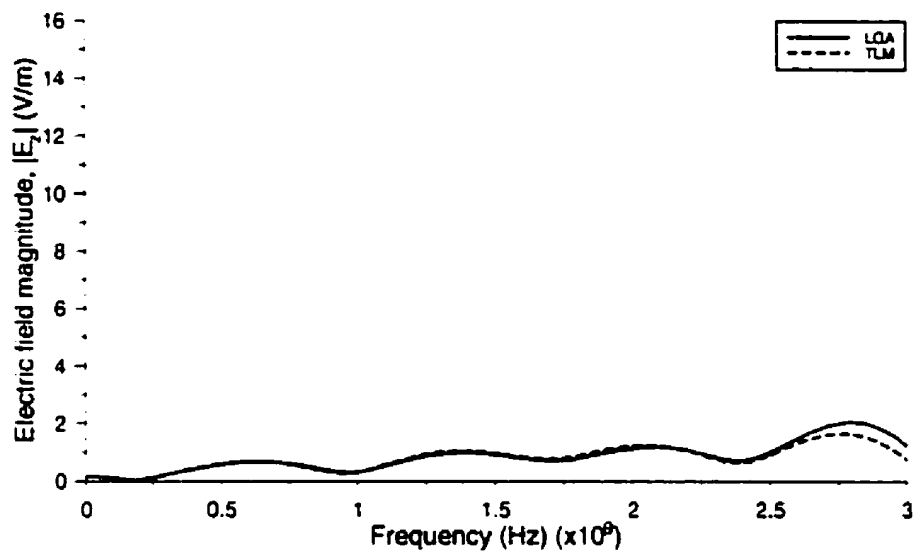

(c)

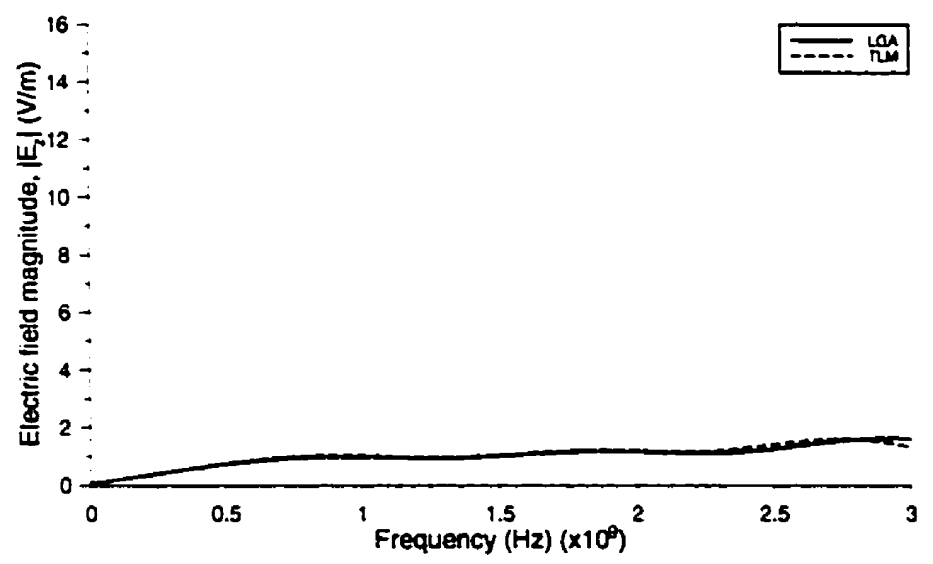

Figure 4.29: Response of the backward scatter in window I to the incident pulse for cylinder cross-sectional area of (a) $60 \Delta X$ 1204, (b) $1404 X$ 1204 , and (c) $604 \times 240 \Delta$. 
(a)

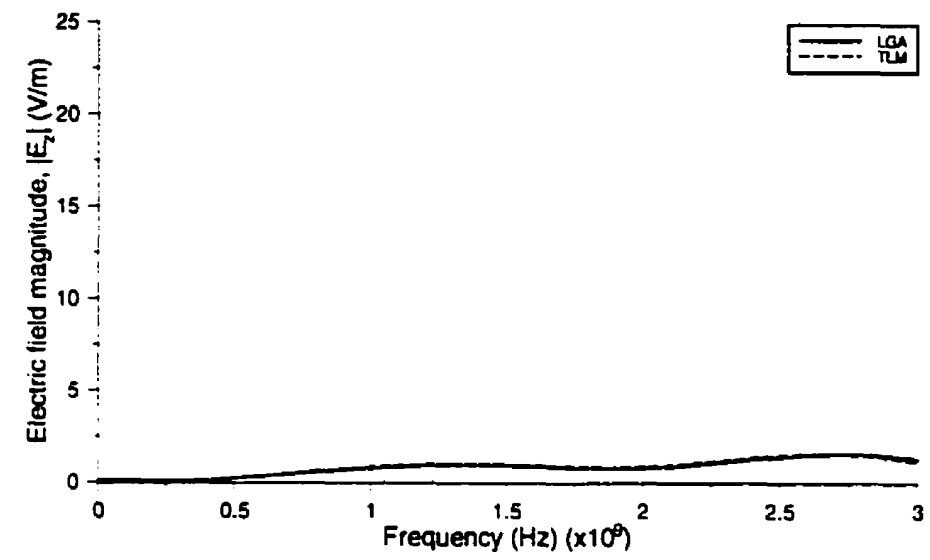

(b)

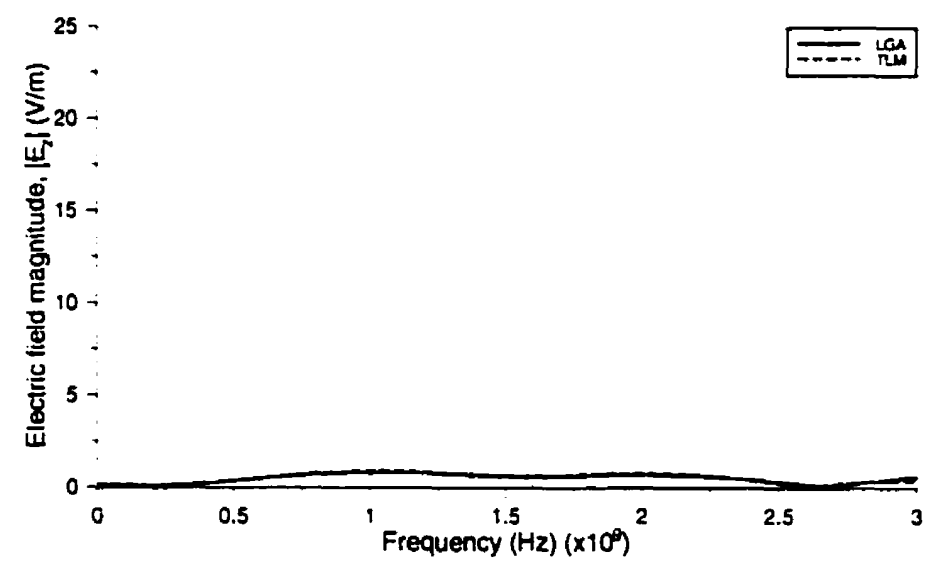

(c)

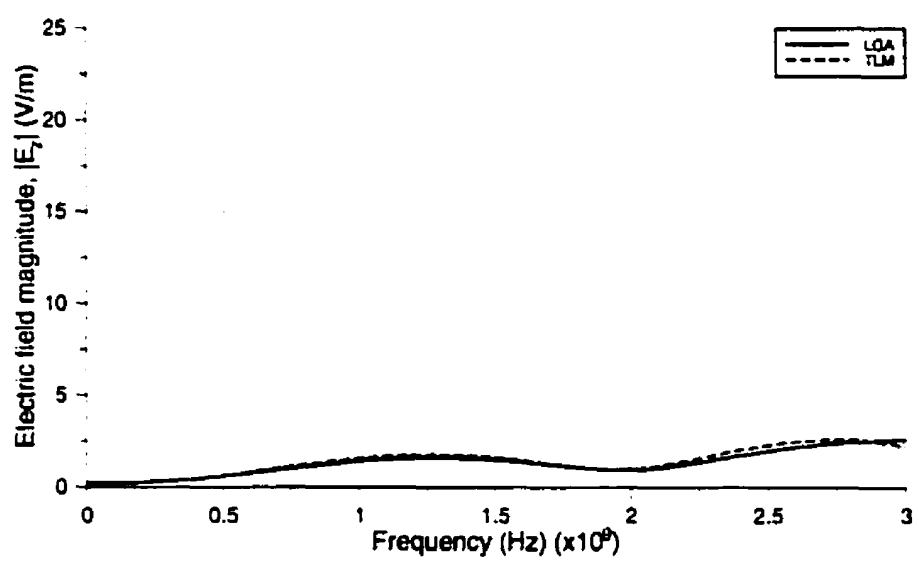

Figure 4.30: Response of the forward scatter in window 2 to the incident pulse for cylinder cross-sectional area of (a) 604X 1204, (b) $1404 X 1204$, and (c) 604X240د. 


\subsubsection{Summary of Results}

The window size had an effect on the noise level and field levels of the peaks and nulls of the time domain waveforms of the $60 \Delta \times 120 \Delta$ scattering experiment. This confirmed discussions presented in section 3.4 regarding the relationship of window size to noise and the averaging effect of the window. The frequency response bandwidth was greater for simulations with smaller window sizes. This was introduced in section 3.5.1, and is due to a widening of the of the sinc function with a decrease in the gate/window width.

From the experiments involving varying dimensions for the scattering object, it was noted that the greater the cross-sectional length in the $y$-direction, the more the scattering object resembles a true PMC wall. This provoked an increase in the level of the backward scattering observed in the time domain. The presence of the cylinder also had a significant effect on the resonant frequencies of the system. Larger cross-sectional width appeared to generate a greater number of resonant peaks in the frequency domain. It is difficult, however, to correctly anticipate the distribution of the wave inside the mesh. Had the cylinder been infinitely thin, the forward and backward scatter patterns would be relatively simple to monitor and predict. Due to the interaction of its two front and back faces, a thicker object has a more complex scattering pattern. This is somewhat evident when comparing the frequency characteristics of the $140 \Delta \times 120 \Delta$ cylinder to those of the other two objects of thinner width. In contrast, a longer strip results in a greater amplitude level for the forward and backward scatter fields. However, these phenomena are not very clearly apparent from the waveforms produced by the two mesh windows. As stated in section 4.2.2.1, one cannot make a simple conclusion regarding the forward and backward scattered radiated fields, without observing the complete scattered pattern as a function of angle around the object.

In both the window and cylinder experiments, LGA waveforms were in agreement with the TLM traces. The frequency domain plots were also comparable for the two computational methods. Remaining discrepancies in the frequency domain can be attributed to errors due to noise and viscosity in the LGA system. 


\section{Chapter 5}

\section{DESIGN OF A MICROWAVE TRANSMISSION LINE FILTER}

This chapter details the design and simulation of a first order microwave filter. The initial design was confirmed with the microwave circuit software package, Libra, and then simulated in the time domain using the HPP-LGA algorithm and TLM method. The LGA method was implemented at various stages of the design procedure to assess the capability of the HPP-LGA algorithm in providing adequate information regarding the filter performance in the time and frequency domains.

\subsection{Sample Microstrip Filter Design}

A narrowband band-stop filter was designed using only shunt transmission line branches, modelling the network structure shown in Figure 5.1. The network branches are realized using transmission line stubs connected to the main transmission line, and having an electrical length of just less than $90^{\circ}$ [40]. Each branch has an impedance $Z_{n}$ equivalent to $Z_{o}=46.3 \Omega$

The filter was designed for a substrate material of thickness $1.59 \mathrm{~mm}\left(1 / 16^{\prime \prime}\right)$ and a relative dielectric constant of $\epsilon_{r}=2.54$. The design frequency was selected to be $1.0 \mathrm{GHz}$, for which the transmission line width corresponding to a $46.3 \Omega$ characteristic impedance is $w=5.0 \mathrm{~mm}$. The stub was assigned a length of $48 \mathrm{~mm}$, slightly less than a quarter wavelength, to account for fringing at the open circuit end of the stub. A single stub transmission line filter was designed using the software package Libra, with the dimensions shown in Figure 5.2. The filter $S_{11}$ and $S_{21}$ responses are shown in Figure 5.3. 


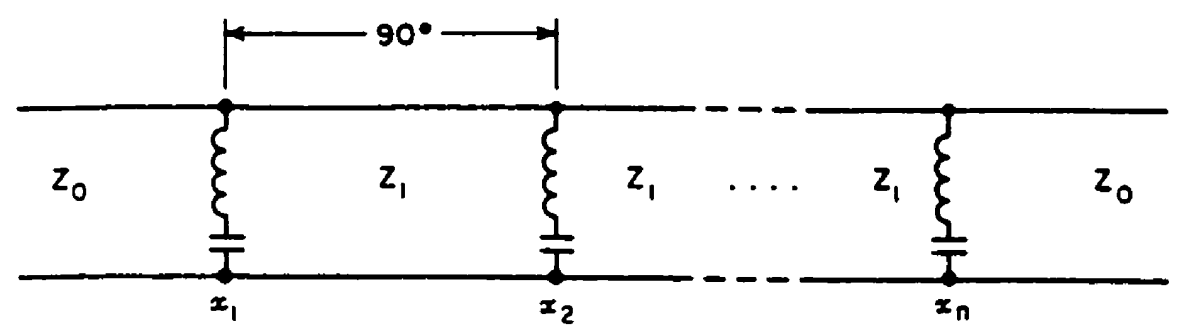

Figure 5.1: Filter network structure [40].

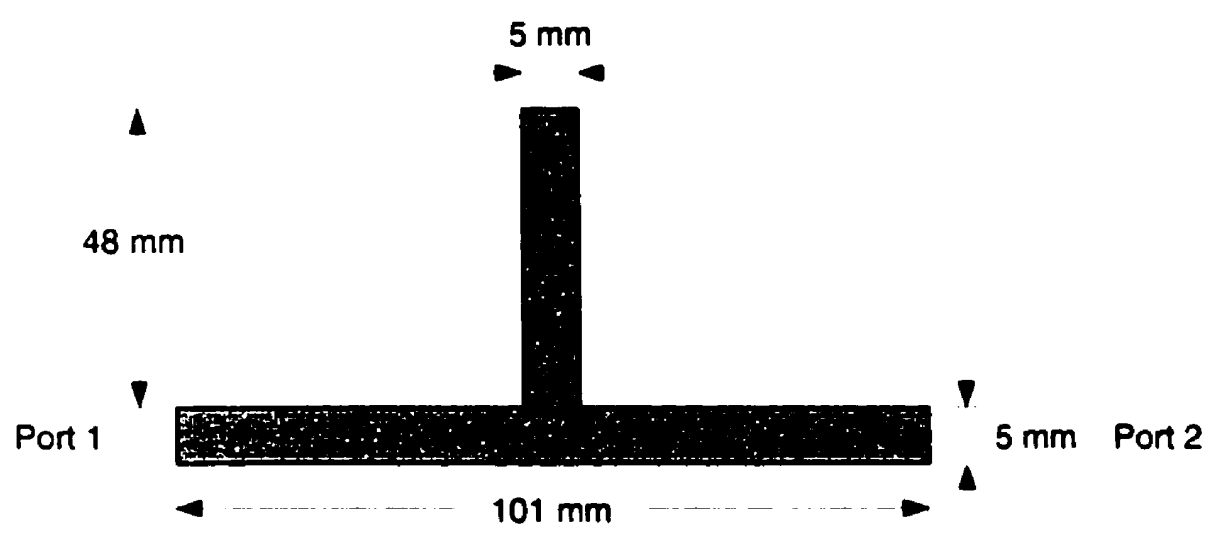

Figure 5.2: Single stub transmission line filter design. 


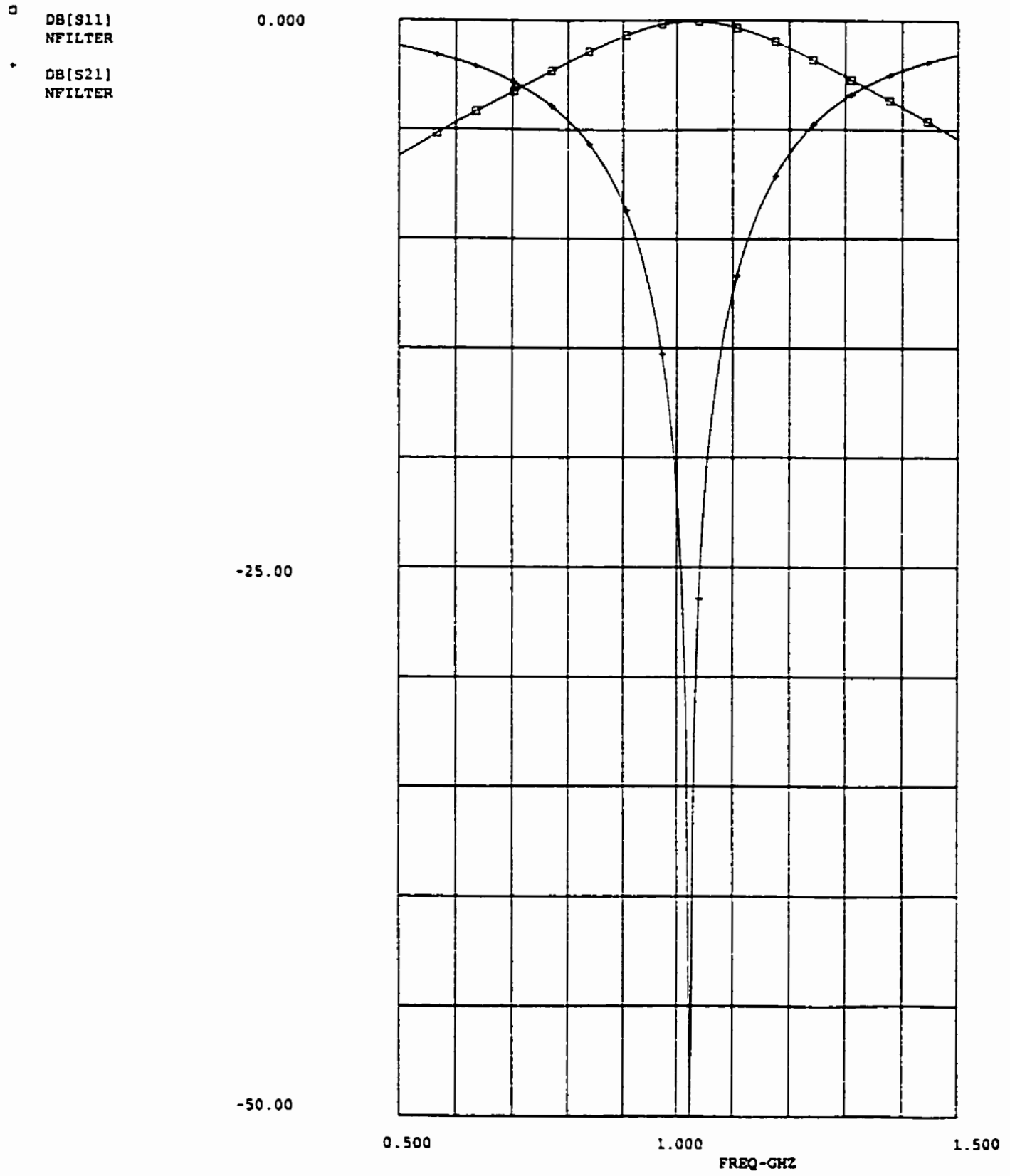

Figure 5.3: Simulation of transmission line filter using Libra. 


\subsection{Simulation of a Transmission Line Model}

The first order filter designed in the previous section was simulated in two stages beginning with the filter main line. The mesh selected for simulation was given dimensions of $1011 \Delta x \times 71 \Delta y, \Delta x=\Delta y=\Delta$, where $\Delta=0.1 \mathrm{~mm}$. The transmission line was centered in the mesh and assigned a width of 50 cells or $5.0 \mathrm{~mm}$, as shown in Figure 5.4. The line ends were terminated by an absorbing boundary. However, the left end $A B C$ was delayed, imposed after $t=170 \Delta t$ iterations, to allow for the propagation of the pulse, as discussed in section 3.2.2. The upper and lower perimeters of the line were assigned PMC boundaries, as was the left line end during the delay period. A Gaussian pulse was launched at $x_{o}=1 \Delta x$ in the mesh with a width of $\sigma=40 \Delta x$ and an amplitude $\rho_{\mathrm{m}}=0.2$, over a background particle distribution with a particle density of $\rho_{0}=0.5$.

Two sampling windows, or probes, were placed on the line, at $x=266 \Delta, y=36 \Delta$, and $x=746 \Delta, y=36 \Delta$. Each window was assigned dimensions of $50 \Delta \mathrm{X} 50 \Delta$. The time-domain output resulting from this simulation, shown in Figure 5.5 (a), indicates a high level of noise. As discussed in section 3.5 , the noise level can be decreased by increasing the window size. However, as the transmission line restricts the dimensions of the sampling window in the $y$ direction, it was decided to stretch the window along $x$, to reduce the noise level and allow for some smoothing of the excited signal. Five additional windows were implemented with dimensions of $60 \times 50,80 \times 50,100 \times 50,120 \times 50$, and $140 \times 50$, centered at the original coordinates. Their respective outputs are plotted in Figures $5.4(\mathrm{~b})$ to $(\mathrm{f})$.
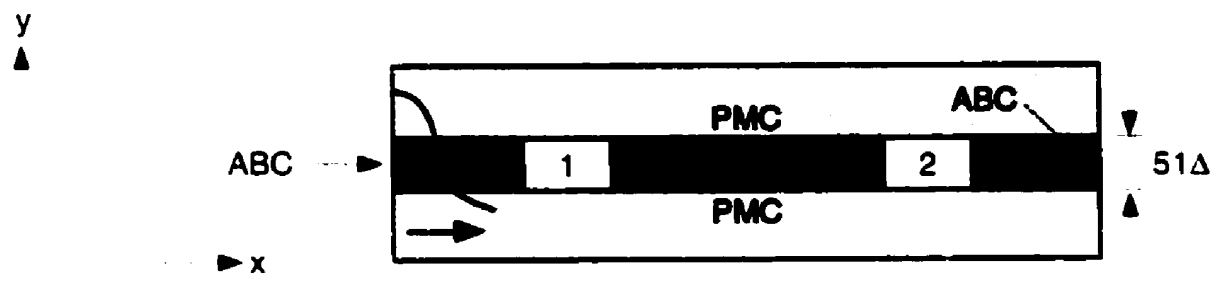

$1011 \Delta$

Figure 5.4: Transmission line configuration for LGA simulation. 
(a)

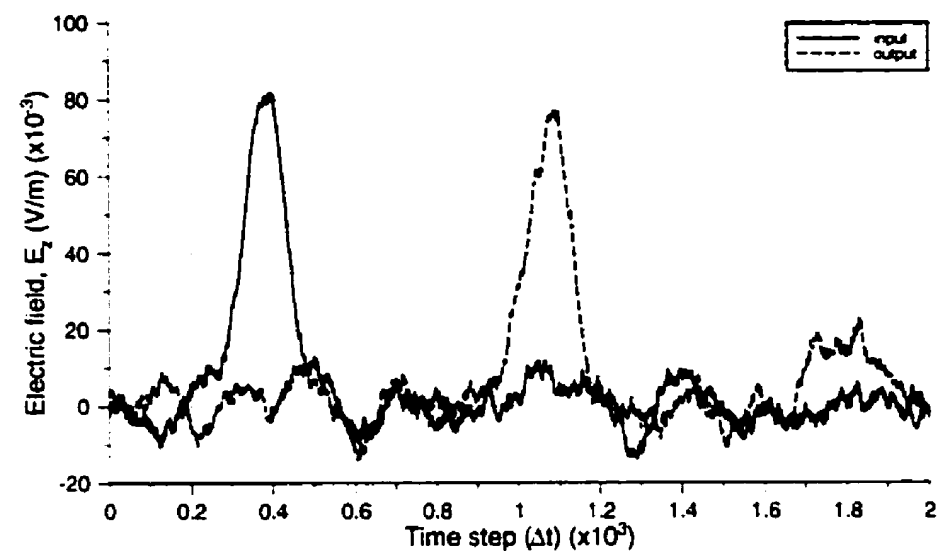

(b)

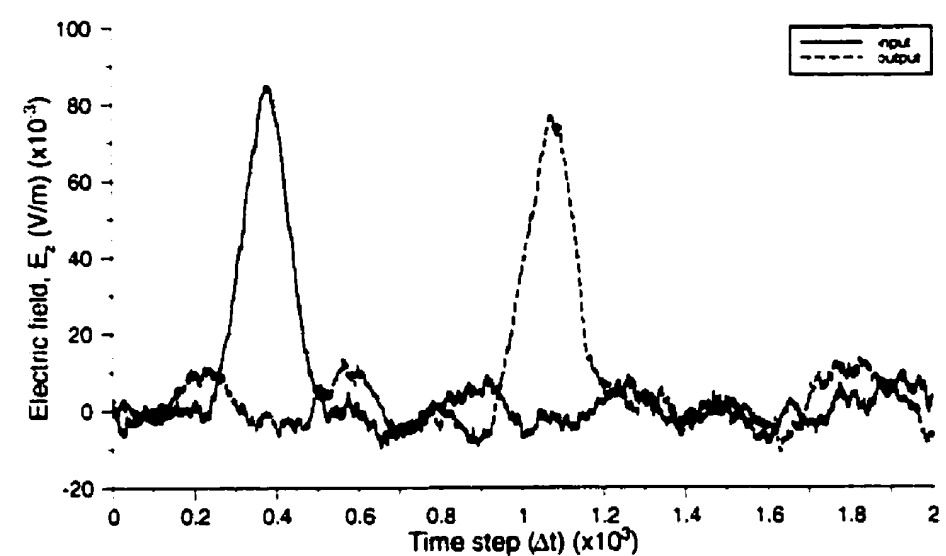

(c)

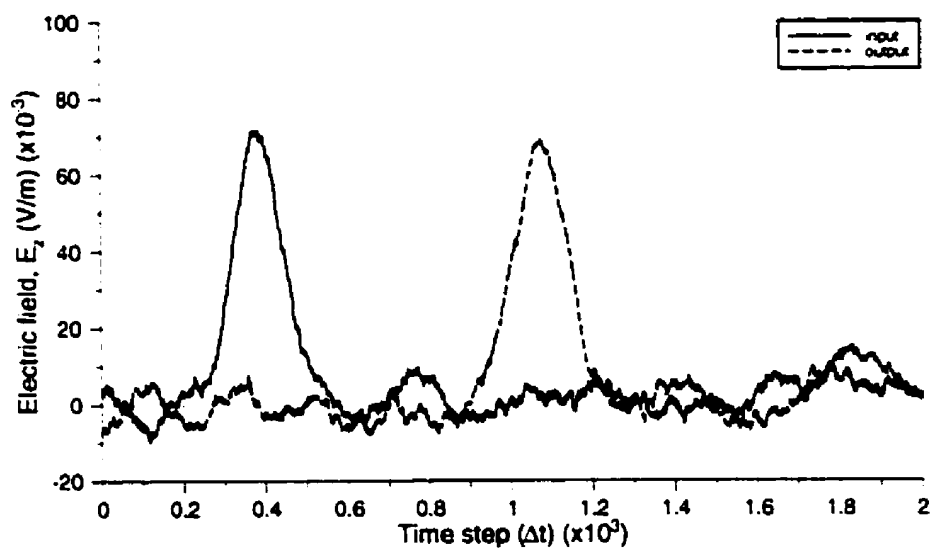

Figure 5.5: Time domain waveforms for a single transmission line with rectangular window dimensions of (a) $50 \times 50$, (b) $60 \times 50$, (c) $80 X$ 50, (d) $100 \times 50$, (e) $120 \times 50$, and (f) $140 \times 50$. 
(d)

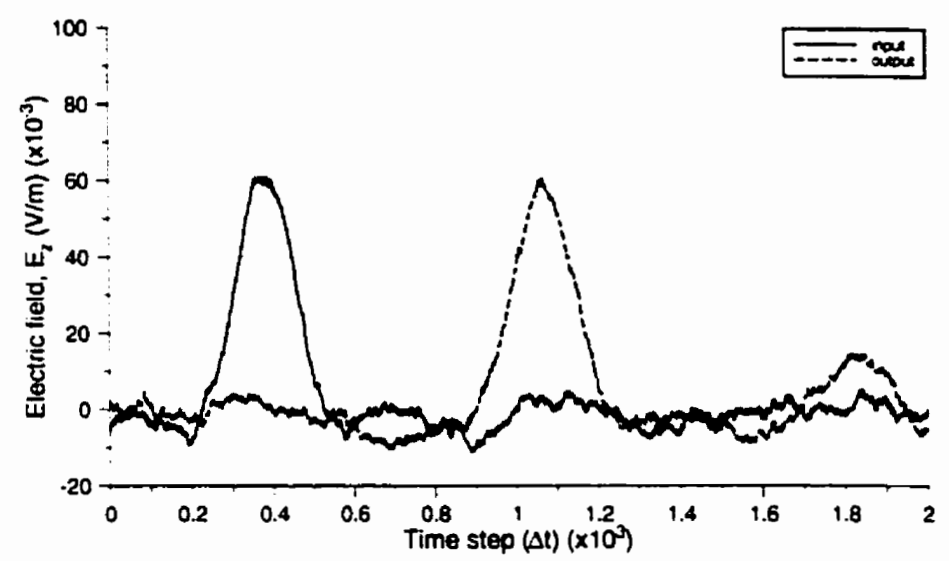

(e)

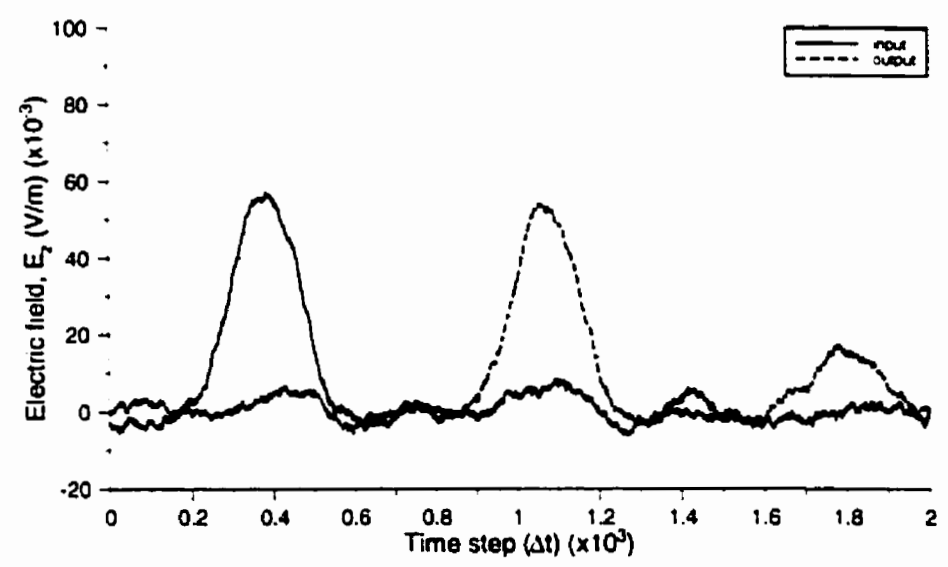

(f)

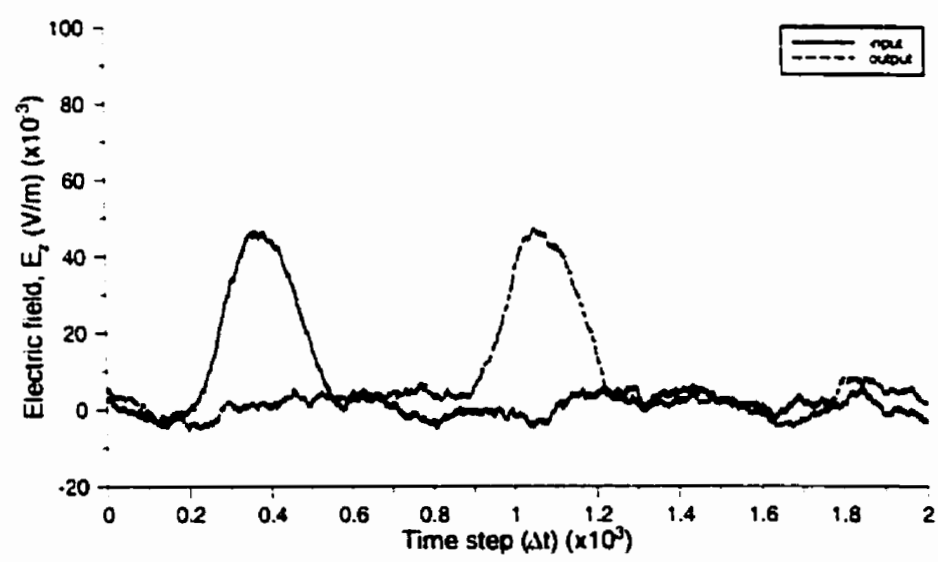

Figure 5.5 (continued) 
As expected, the overall pulse undergoes a smoothing effect. A reflection resulting from the poor accuracy of the right absorbing boundary termination is observed in the second window at approximately $t=1800 \Delta t$ in Figures 5.5 (c) and (d). This reflection is less noticeable for smaller window sizes due largely to the higher degree of noise. It is also less visible for the largest window size of $140 \Delta \mathrm{X} 50 \Delta$. The evidence of the reflection in this window may be somewhat lost due to increased damping of the pulse amplitudes. Since these time-domain responses are the result of single simulations and not averaged data from a number of runs, differences observed between each case may be due to the random nature of particle selection within the mesh. The $120 \Delta \mathrm{X} 50 \Delta$ window was selected for its reduced noise level in comparison to smaller windows, as well as for its seemingly clearer depiction of the original Gaussian pulse and the reflection by the absorbing boundary. A representation of the input signal level, the actual observed pulse by this window for one simulation, and the theoretical rms noise level are shown in Figure 5.6.

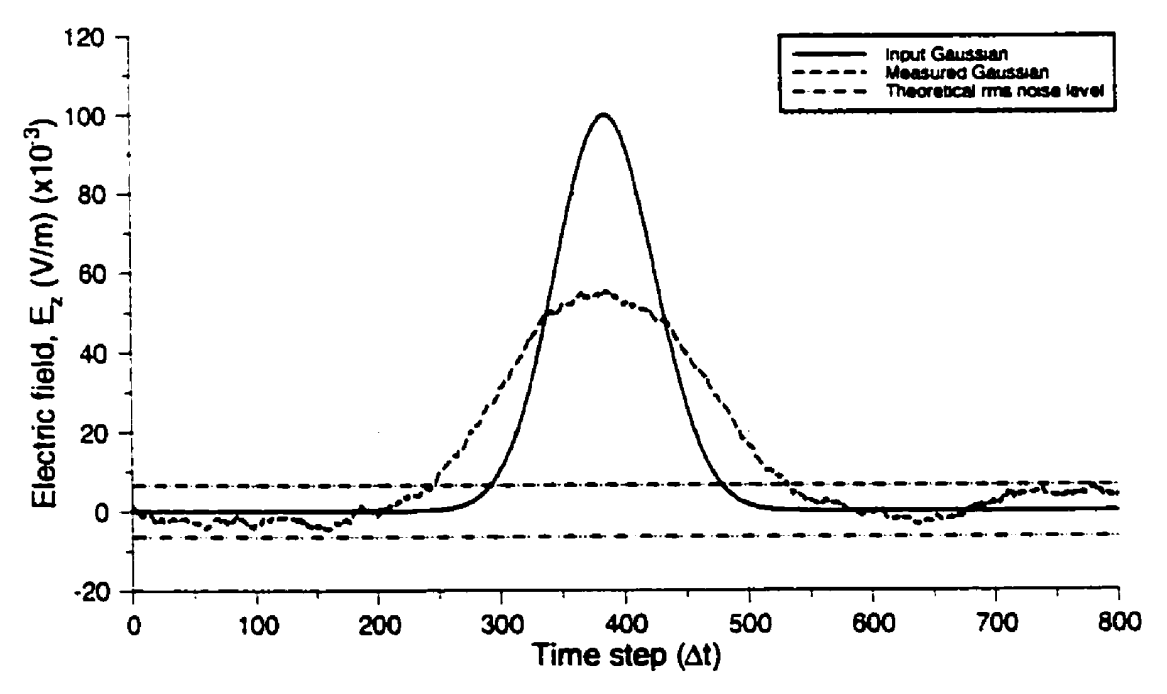

Figure 5.6: Representation of the input Gaussian signal, the actual pulse observed by the $1204 X 504$ window, and the theoretical rms noise level. 
The time domain response of the $1011 \Delta \times 51 \Delta$ transmission line with two rectangular sampling windows of size $120 \Delta \mathrm{X} 50 \Delta$ was computed and averaged for ten differing random number generator seeds. The resulting electric field at each window are shown in Figures 5.7 (a) and (b) for 5000 iterations. The reflection caused by the $A B C$ at the right end of the line is illustrated at $t=2518 \Delta t, t=1828 \Delta t$, for windows 1 and 2 , respectively. There is a noticeable improvement in the noise level of the averaged 10 timedomain runs in comparison with the single run shown in Figure 5.5 (e).

As in section 3.2.2, the reflection coefficient of the $\mathrm{ABC}$ reflection to the transmitted pulse in window 2 can be computed using (3.3) and (3.4). Taking the transmitted pulse and the $A B C$ reflection observed at window $2,0.0538 \mathrm{~V} / \mathrm{m}$ and $0.0112 \mathrm{~V} / \mathrm{m}$ respectively, a reflection coefficient of $-14 \mathrm{~dB}$ was determined. The $\mathrm{ABC}$ at the left end of the line also sends a reflection back into the mesh, but it is not visible in the ensemble averaged data in Figure 5.7. For the computed reflection coefficient of the $\mathrm{ABC}$ boundary, a pulse with an amplitude of $0.002 \mathrm{~V} / \mathrm{m}$ should be observed at approximately $3235 \Delta t$. However, the theoretical rms noise level for a $120 \Delta \mathrm{X} 50 \Delta$ window is $0.0065 \mathrm{~V} / \mathrm{m}$, as shown in Figure 5.6. Thus, the ABC reflection due to the left wall is lost below the noise level in the system. 


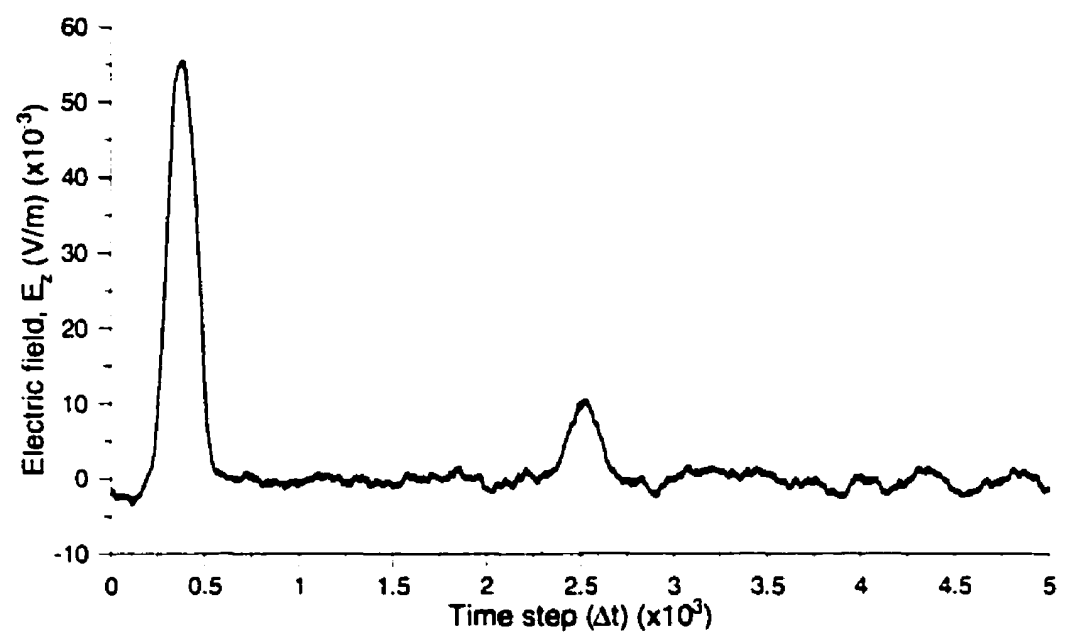

(a)

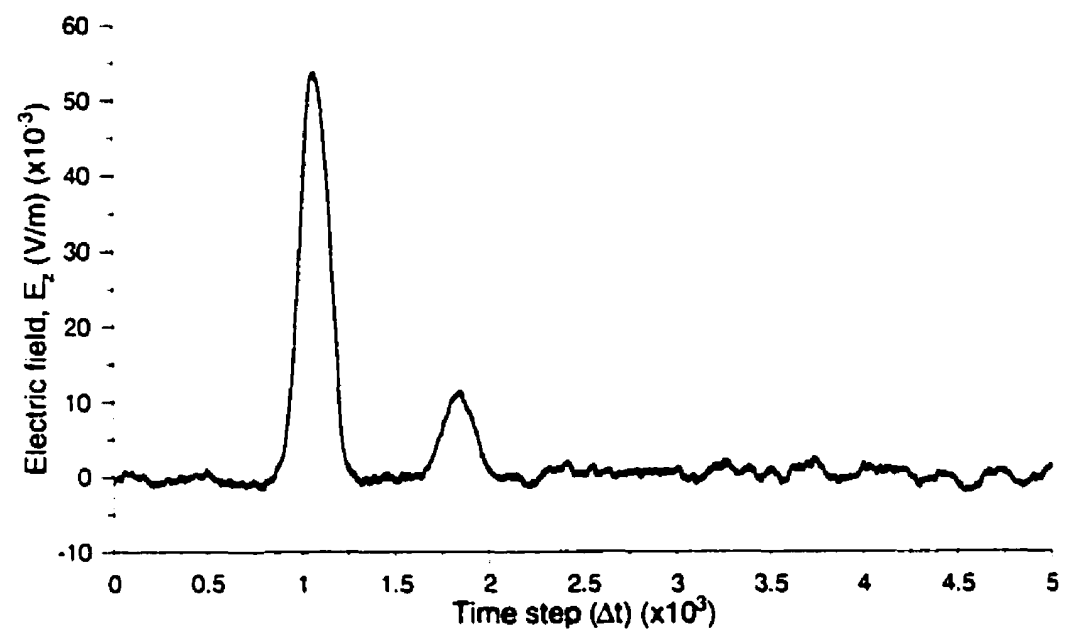

(b)

Figure 5.7: Time-domain output of a 10 run ensemble average for a transmission line simulation with two $1204 X 50 \Delta$ windows; (a) window 1 , (b) window 2. 


\subsection{Simulation of a First Order Filter}

\subsubsection{Time Domain}

\subsubsection{LGA and TLM Configurations}

The LGA filter design was realized by adding a transmission line stub to the filter main line. The mesh size was increased to $1011 \Delta \times 581 \Delta$ to accommodate the $\lambda / 4$ stub and maintain the parameters of the design outlined in Figure 5.2. The mesh cell size remained at $\Delta=0.1 \mathrm{~mm}$. The $120 \Delta \times 50 \Delta$ windows along the main line were maintained at their original locations along $x$, now at $(266 \Delta, 51 \Delta)$, and $(746 \Delta, 51 \Delta)$. A third window was placed in the stub at $(506 \Delta, 316 \Delta)$, shown in Figure 5.8.

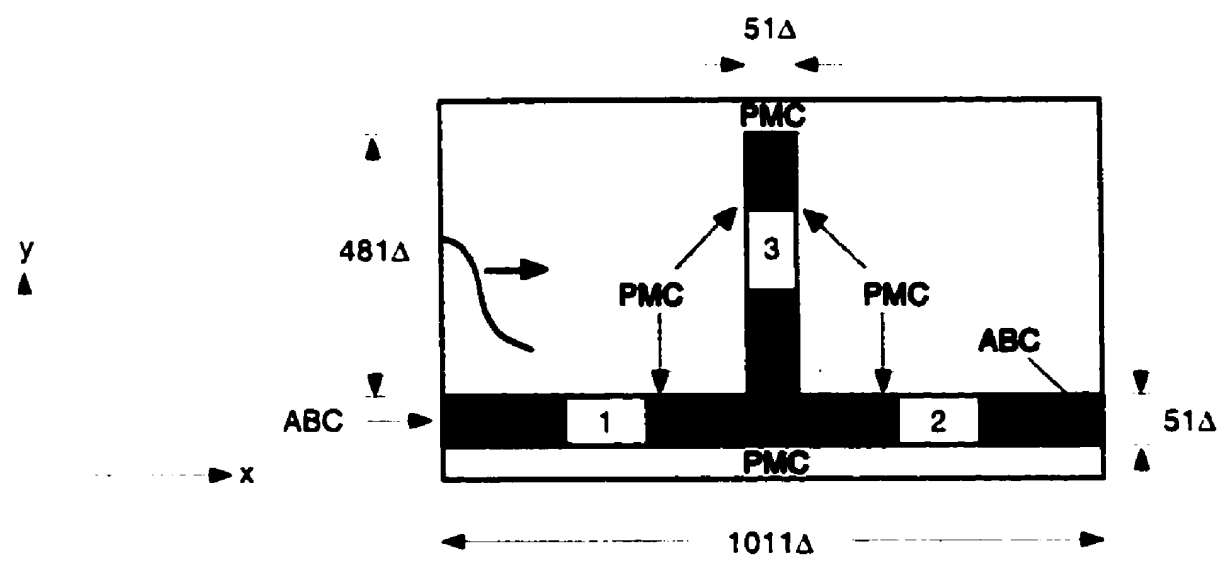

Figure 5.8: Filter configuration for LGA simulation. 
The identical full-scale mesh configuration was simulated by the TLM method for 5000 times steps. The window diameter of $120 \Delta$ was equated to the length of the LGA rectangular sampling window. However for these dimensions, the rectangular window area is greater than the circular window area by a factor of 0.53 . The time domain waveforms for the input, output and stub windows given in Figures 5.10 (a) to (c), respectively, have been scaled by this amount.

The window geometry selected overlaps the transmission line boundaries, since the diameter is greater than the line width of $50 \Delta$. The wave excitation is launched at each cell of the column associated with $x_{o}=1 \Delta$. Sampling the TLM node impulses outside the line would give an incorrect computation of the field behaviour at each window. A PMC barrier was placed in the mesh region external to the transmission line at $x=200 \Delta$, as shown in Figure 5.9. This effectively limits the wave propagation in the mesh outside of the filter transmission lines to a region between the leftmost mesh boundary and the PMC barrier. Thus, the overlapped region of the circular window only samples the wave behaviour of the transmission line. Contrasts in pulse amplitudes are therefore attributed to the slight differences in the number of averaged cells of the circular window in comparison to the rectangular geometry.

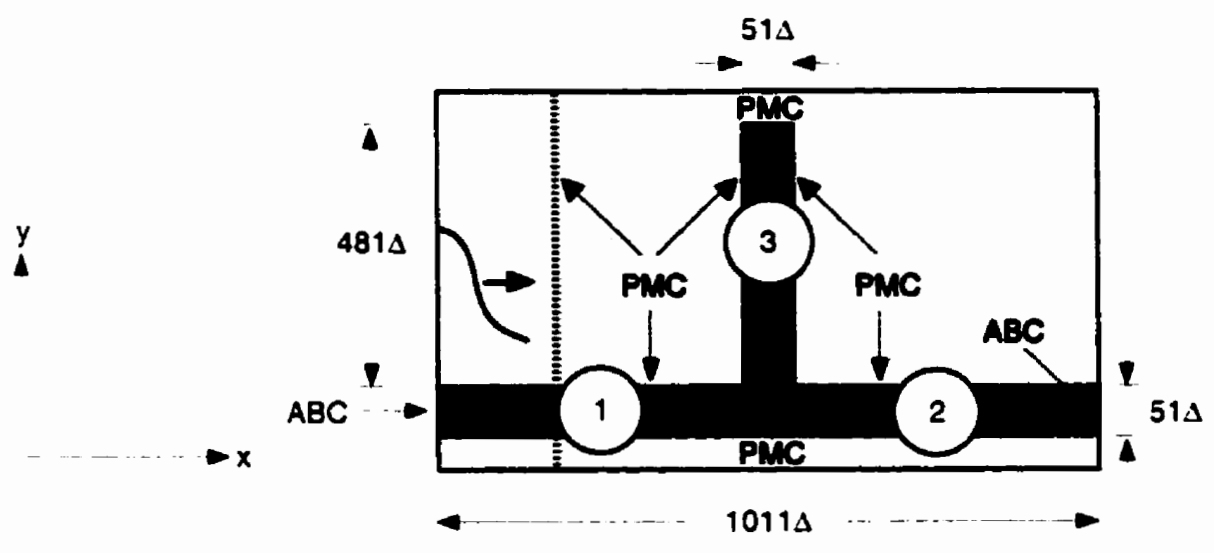

Figure 5.9: Filter configuration for TLM simulation. 


\subsubsection{Results}

The time-domain output of each window for an ensemble average of 10 simulated runs is shown in Figures 5.10 (a) to (c) over 5000 iterations. The filter excitation and the boundary conditions of the main line are identical to those outlined in section 5.2. The walls of the stub branch were assigned PMC boundaries.

The incident pulse observed initially by window 1 at $t=376 \Delta t$ has a computed amplitude of $0.055 \mathrm{~V} / \mathrm{m}$. Upon arriving at the stub junction, the pulse "sees" an impedance mismatch at this point. Ideally, a portion of the wave is reflected back towards the main line input, and the remaining signal is transmitted equally to the stub and down the right end of the main line. Because of the positional symmetry of the transmission line windows, the reflection and transmissions resulting from the impedance mismatch at the junction are observed at approximately the same time by each window, around $t=1055 \Delta t$.

The portions of the wave that are transmitted and reflected can be easily determined by transmission line theory. The reflection coefficient is computed as,

$$
\Gamma=\frac{Z_{L}-Z_{o}}{Z_{L}+Z_{0}}
$$

The transmission coefficient is related to $\Gamma$ by the following equation,

$$
\tau=1+\Gamma
$$

Both the stub and the main line have a width corresponding to a $46 \Omega$ line at the design frequency. The impedance $Z_{\text {junction }}$ at their intersection is therefore,

$$
Z_{\text {junction }}=\frac{Z_{o} Z_{l}}{Z_{o}+Z_{l}}=23 \Omega
$$

where $Z_{l}$ and $Z_{o}$ represent the stub and line impedances, respectively. From (5.1), this results 
in a reflection coefficient of $\Gamma=-1 / 3$. The transmission coefficient corresponding to the junction becomes $\tau=2 / 3$. Therefore, $2 / 3$ of the original excitation is expected to be transmitted into the stub and towards the right end of the main line, while $-1 / 3$ of the original pulse will be reflected back towards the left end of the filter main line.

At $t=1055 \Delta t$, an electric field level of $-0.017 \mathrm{~V} / \mathrm{m}$ is computed by window 1 , resulting from the reflection at the stub junction. The $0.038 \mathrm{~V} / \mathrm{m}$ pulse in window 2 represents the portion of the original Gaussian excitation transmitted down the main line, while window 3 details the transmission of a $0.035 \mathrm{~V} / \mathrm{m}$ pulse along the stub. Upon addition, their amplitudes make up the original incident pulse. The observed and ideal pulse amplitudes resulting from the first mismatch event are listed in Table 5.1.

Table 5.1: Computed and predicted pulse amplitudes in V/m at windows 1,2 , and 3 (input, output, and stub) for the first-order mismatch.

\begin{tabular}{|c|c|c|}
\hline Filter port & 1 $^{\text {st-order mismatch }}$ & Ideal \\
\hline Input & -0.017 & 0.018 \\
\hline Output & 0.038 & 0.037 \\
\hline Stub & 0.035 & 0.037 \\
\hline
\end{tabular}

Due to their equivalent line impedances, the transmission into the stub branch and the main line output, intuitively, should have identical amplitudes. However, a slight difference was observed, which can be attributed partly to the randomness of the particles in the LGA mesh. Also, the stub and main line are not composed of a single column of cells and thus do not meet at a single point. Because of the finite width of the line, i.e. the number of cells through the junction, the amount of signal transferred to each branch may not correspond exactly to that calculated from (5.1) and (5.2).

Because the stub is not terminated by an $\mathrm{ABC}$, the signal transmitted into the stub branch is bounced-back towards the junction by the stub PMC boundary. The returning 
pulse is observed at $t=1770 \Delta t$ in Figure $5.10(\mathrm{c})$. The signal then undergoes a reflection back into the stub and a transmission into both ends of the mainline upon encountering the junction mismatch a second time. This phenomena continues until the signal decays below the noise level in the system. For the iteration period shown in Figures 5.10 (a) to (c), the effects can be traced for three mismatch events at the filter junction.

Of serious concern are the $\mathrm{ABC}$ reflections propagating down the line due to the poor accuracy of the $A B C$ at both ends. The reflections by the east and west $A B C$ are first observed at approximately $t=1800 \Delta t$ in windows 1 and 2 of Figure 5.10 (a) and (c). It is a simple matter to trace the first order mismatch of the system since no $A B C$ reflections have been released into the filter main line. However, it is more difficult to observe and account for the effects of further mismatch events as the wave propagation along the line becomes more complex. The wave corresponding to the original signal and those resulting from the two $\mathrm{ABC}$ at the line terminations interact simultaneously, affecting the dynamics of the of the reflections and transmissions at the stub junction. It must be noted that in window 1 , a negative reflection was returned by the first mismatch event. It is that negative reflection which is re-introduced along the main line with a reduction in magnitude equivalent to $\Gamma=$ $-14 \mathrm{~dB}$, as observed in the section 5.2. The pulse reflected at window 2 underwent a similar amplitude reduction.

Table 5.2 details the transmitted and reflected pulse amplitudes corresponding to each window for the second and third order mismatches, as well as their ideal values, neglecting reflections by the $A B C$ walls. The observed and ideal pulse amplitudes are close, but nevertheless show some differences due to the $A B C$ wall reflections.

Tables 5.3 and 5.4 list the first and second $\mathrm{ABC}$ reflections introduced into the system, observed at approximately $t=1800 \Delta t$, and $t=3200 \Delta t$, respectively. As with the original excitation, the $\mathrm{ABC}$ reflections undergo a transmission into the stub and opposing line end, as well as a reflection back towards their respective $A B C$ port. This impedance mismatch is also detailed in Tables 5.3 and 5.4. 
Table 5.2: Observed and ideal pulse amplitudes in $\mathrm{V} / \mathrm{m}$ at windows 1,2 , and 3 (input, output, and stub) for the second and third order mismatches.

\begin{tabular}{|c|c|c|c|c|}
\hline Filter port & $\begin{array}{c}2^{\text {nd }} \text { order } \\
\text { mismatch }\end{array}$ & Ideal* & $\begin{array}{c}3^{\text {rd }} \text { order } \\
\text { mismatch }\end{array}$ & Ideal* $^{*}$ \\
\hline Input & 0.0274 & 0.0234 & -0.0052 & -0.0078 \\
\hline Output & 0.0162 & 0.0234 & -0.0037 & -0.0078 \\
\hline Stub & -0.0091 & -0.0117 & 0.0084 & 0.0039 \\
\hline
\end{tabular}

* Neglecting reflection effects from $A B C$ walls.

Table 5.3: Amplitudes in $V / m$ of the $A B C$ reflections at windows $I$ and 2 (input and output) introduced after the first $A B C$ reflection, and their resulting mismatch at the stub junction.

\begin{tabular}{|c|c|c|c|}
\hline \multirow{2}{*}{ Filter port } & \multirow{2}{*}{$1^{\text {st }} \mathrm{ABC}$ reflection } & \multicolumn{2}{|c|}{ Mismatch due to $\mathrm{ABC}$ reflection } \\
\cline { 3 - 4 } & & Reflection & Transmission \\
\hline Input & -0.0042 & 0.0014 & -0.0028 \\
\hline Output & 0.0092 & -0.0031 & 0.0061 \\
\hline
\end{tabular}

Table 5.4: Amplitudes in $V / m$ of the $A B C$ reflections at windows $I$ and 2 (input and output) introduced after the second $A B C$ reflection, and their resulting mismatch at the stub junction.

\begin{tabular}{|c|c|c|c|}
\hline \multirow{2}{*}{ Filter port } & $2^{\text {nd }}$ & $\mathrm{ABC}$ reflection & \multicolumn{2}{|c|}{ Mismatch due to $\mathrm{ABC}$ reflection } \\
\cline { 3 - 4 } & & Reflection & Transmission \\
\hline Input & 0.0057 & -0.0019 & 0.0038 \\
\hline Output & 0.0046 & -0.0015 & 0.0031 \\
\hline
\end{tabular}


Table 5.5 summarizes the mismatch at the filter junction that occurs as a result of the $\mathrm{ABC}$ reflections listed in the of Table 5.3. Also provided in the table are their effects on the dynamics of the second-order mismatch. Table 5.6 gives similar information with respect to Table 5.4, corresponding to the third-order mismatch. In both Tables 5.5 and 5.6, the transmissions and reflections resulting from the $\mathrm{ABC}$ reflection, when summed with the ideal signal amplitudes for each mismatch, are comparable to the actual observed event.

Table 5.5: $\quad$ Correction of reflected and transmitted signal by contribution of the first $A B C$ reflections in $\mathrm{V} / \mathrm{m}$ at the three port windows.

\begin{tabular}{|c|c|c|c|c|c|}
\hline Filter port & \multirow{2}{*}{$\begin{array}{c}2^{\text {nd }} \text { order } \\
\text { ideal } \\
\text { mismatch }\end{array}$} & \multicolumn{2}{|c|}{$\begin{array}{c}\text { Mismatch due to } A B C \\
\text { reflection }\end{array}$} & $\begin{array}{c}\text { Ideal }+ \text { ABC } \\
\text { refl. } \\
\text { mismatch }\end{array}$ & $\begin{array}{c}\text { Actual 2 } \\
\text { order } \\
\text { mismatch }\end{array}$ \\
\cline { 3 - 4 } & Left wall & Right wall & & \\
\hline Input & 0.0234 & 0.0014 & 0.0061 & 0.0309 & 0.0274 \\
\hline Output & 0.0234 & -0.0028 & -0.0031 & 0.0175 & 0.0162 \\
\hline Stub & -0.0117 & -0.0028 & 0.0061 & -0.0084 & -0.0091 \\
\hline
\end{tabular}

Table 5.6: $\quad$ Correction of reflected and transmitted signal by contribution of the second $A B C$ reflections in $V / m$ at the three port windows.

\begin{tabular}{|c|c|c|c|c|c|}
\hline Filter port & $\begin{array}{c}3^{\text {rd }} \text { order } \\
\text { ideal } \\
\text { mismatch }\end{array}$ & \multicolumn{2}{|c|}{$\begin{array}{c}\text { Mismatch due to } \mathrm{ABC} \\
\text { reflection }\end{array}$} & $\begin{array}{c}\text { Ideal }+\mathrm{ABC} \\
\text { refl. } \\
\text { mismatch }\end{array}$ & $\begin{array}{c}\text { Actual } 3^{\text {rd }} \\
\text { order } \\
\text { mismatch }\end{array}$ \\
\cline { 3 - 4 } & Left wall & Right wall & & \\
\hline Input & -0.0078 & -0.0019 & 0.0031 & -0.0066 & -0.0052 \\
\hline Output & -0.0078 & 0.0038 & -0.0015 & -0.0056 & -0.0037 \\
\hline Stub & 0.0039 & 0.0038 & 0.0031 & 0.0108 & 0.0084 \\
\hline
\end{tabular}


It should be noted that as the wave propagates, the effects of mesh viscosity, i.e. the damping of the wave, become more significant with time. This may account for the decreased magnitude of the signal at all three windows for the actual mismatch event listed Table 5.6. The transmission of these signals occurs after approximately 4000 iterations and have lesser field levels than the sum of the ideal and $A B C$ reflection values.

The TLM and LGA traces in Figures 5.10 (a) to (c) are relatively similar, with the exception of the lack of $A B C$ reflections in the TLM traces. The TLM algorithm utilizes a more efficient implementation of an absorbing boundary. Thus, ABC reflections do not corrupt the three detectable mismatches. 
(a)

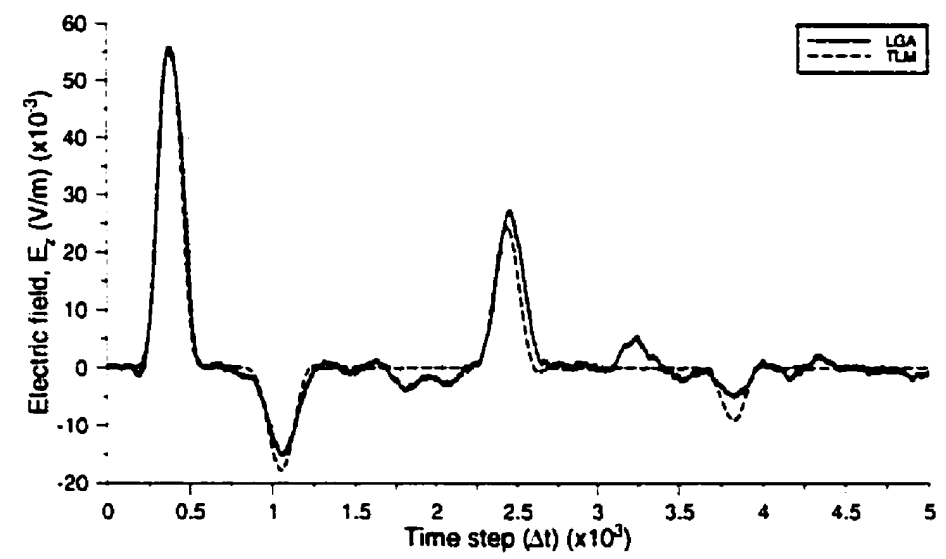

(b)

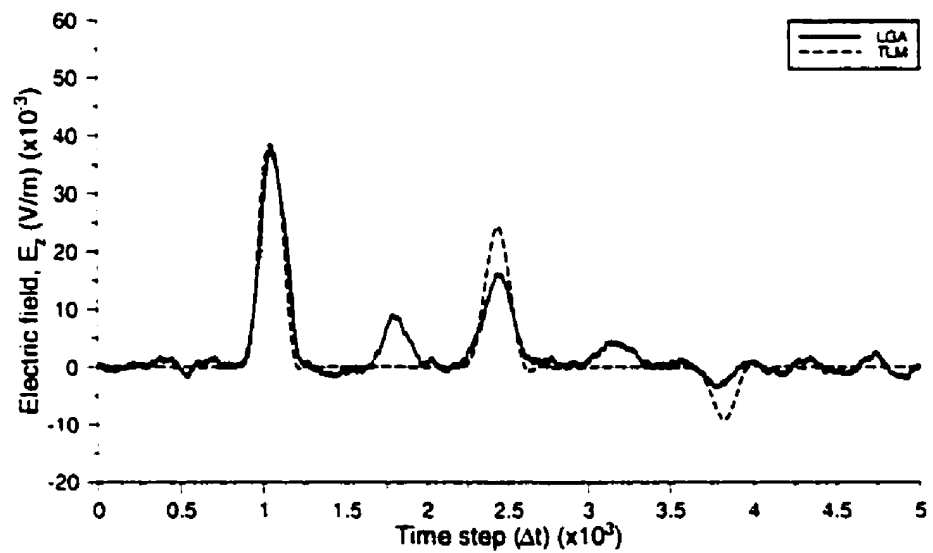

(c)

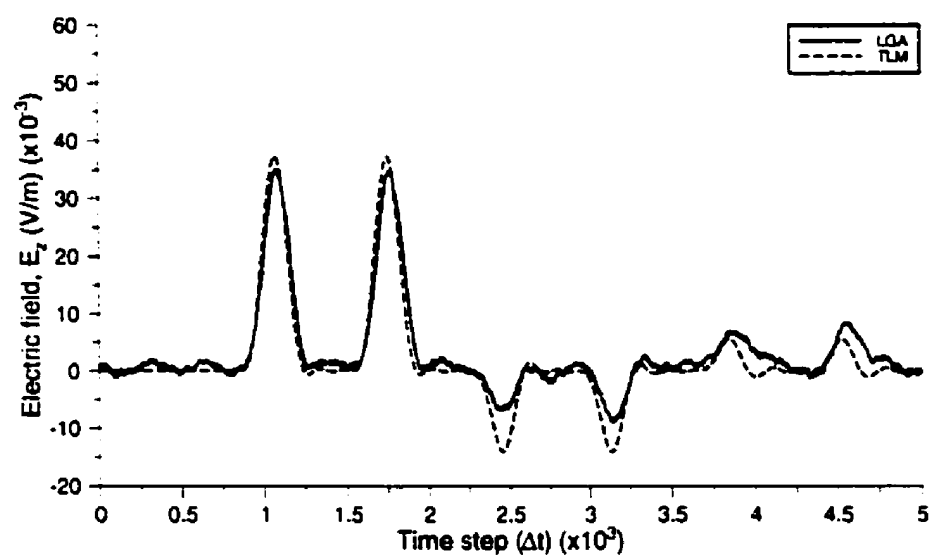

Figure 5.10: Time domain waveforms for (a) input (window 1), (b) output (window 2), and (c) the stub window, for the simulated TLM and LGA filter. 


\subsubsection{Frequency Domain}

The relevant frequency domain information of the filter are the electrical scattering parameters provided by the information at windows 1 and 2. The reflected $\left(S_{11}\right)$ and transmitted $\left(\mathrm{S}_{21}\right)$ power in $\mathrm{dB}$, for the LGA and TLM simulations are given in Figures 5.11 (a) and (b), respectively.

The $S_{11}$ traces in Figure 5.11 (a) are relatively similar, but indicate a slight frequency shift in the TLM case with respect to the design frequency of $1.0 \mathrm{GHz}$. This frequency shift is also noticeable in the TLM $S_{21}$ trace of Figure $5.11(\mathrm{~b})$, where the notch has also shifted with respect to the design frequency. The frequency shift in the TLM $S_{11}$ and $S_{21}$ traces is attributed to contrasts in the analysis methods regarding the TLM algorithm and the Libra software. The TLM algorithm involves a full wave method of analysis, whereas the microwave circuit software Libra is based on simple quasi-statics. This may therefore result in slight differences between the data shown in Figure 5.3 by the Libra simulation, and Figure 5.11 involving the TLM computation.

From Figure 5.11 (b), the level of transmitted power computed by the LGA simulated data at the design frequency is adequate. Consideration was focused on the possible effects of the ensemble averaging on the smoothing of the null in the $S_{21}$ trace. The $S_{11}$ and $S_{21}$ data were computed for the individual LGA filter simulations, with four of these cases shown in Figure 5.12. The $S_{21}$ traces provided in Figure 5.12 (b) clearly display contrasting frequencies, from approximately 0.9 to $1.3 \mathrm{GHz}$, for each individual case. In addition, the transmitted power at each null is considerably different, ranging from a low of approximately $-28 \mathrm{~dB}$ to a high of $-10 \mathrm{~dB}$.

It is possible however, that the poor accuracy of the absorbing boundary in the LGA simulation is affecting the frequency response of the LGA simulations, exclusively or in addition to the ensemble averaging. The line length of the main filter transmission line was extended at both ends to obtain the system reflections and transmissions without the corruptions caused by the $\mathrm{ABC}$ reflections. Specifically, by making the line length longer, the $\mathrm{ABC}$ reflections are not observed in the first and second windows over the computed 
iterations in the time domain. The LGA filter was therefore simulated for a mesh size of $3011 \Delta \mathrm{X} 581 \Delta$, as well $2011 \Delta \mathrm{X} 581 \Delta$. The shift in frequency was still observed, regardless of the line length or absence of $\mathrm{ABC}$ reflections. It would appear that the results are case (i.e. seed) dependent. No observable or predictable trend was found. 


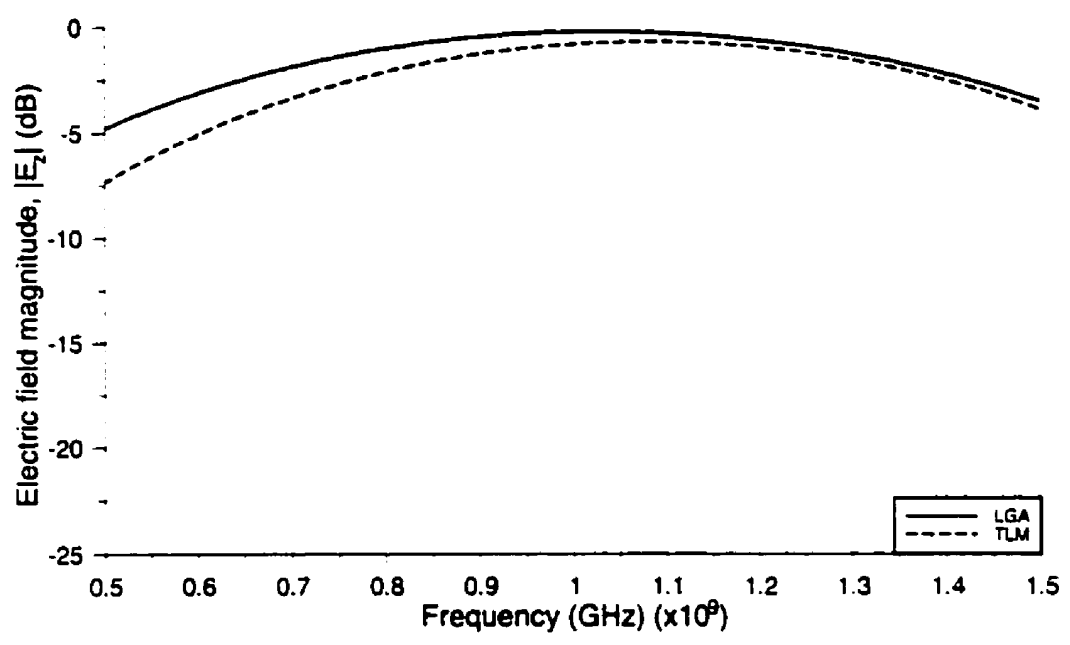

(a)

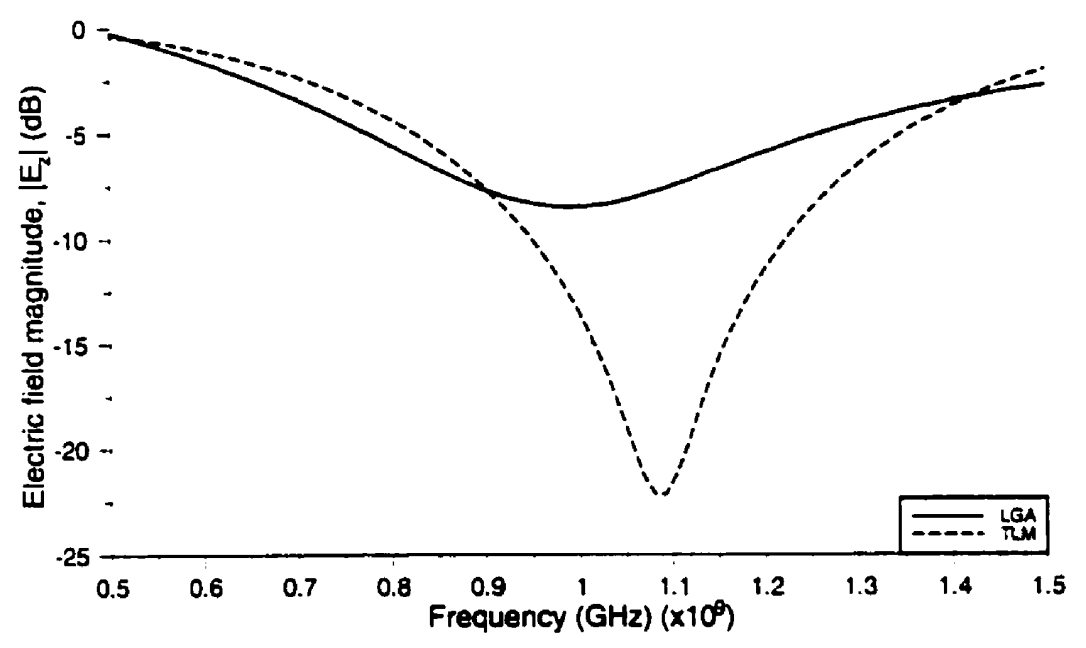

(b)

Figure 5.11: Reflected and transmitted power in $d B$ for LGA and TLM frequency transmission line filter; (a) $S_{1 l}$, and (b) $S_{2 l}$. 


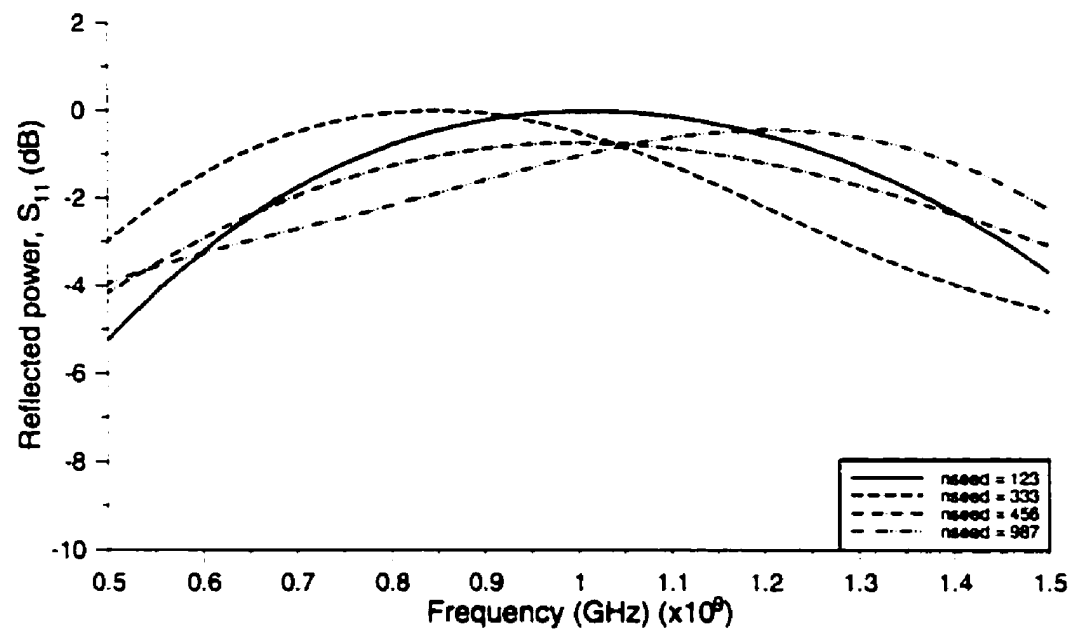

(a)

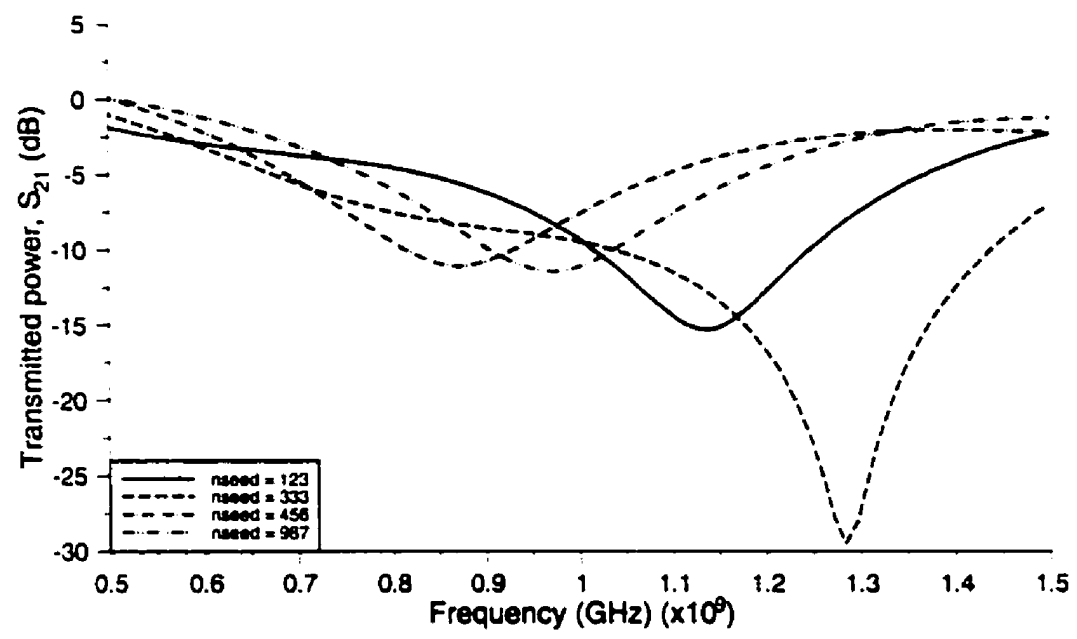

(b)

Figure 5.12: Reflected and transmitted power in $d B$ of four individual $L G A$ seed simulations; (a) $S_{11}$ and (b) $S_{21}$. 


\subsection{Summary of Results}

A simple single stub band reject filter was designed and simulated in two stages beginning with the filter main line using both the TLM and LGA techniques. Initial simulations were performed using only a single transmission line to select a suitable sampling windows dimensions. Consideration was placed on the pulse dynamics as well as the noise level due to the window size. The $120 \Delta \times 50 \Delta$ window was selected for its reduced noise level in comparison to smaller windows, as well as for its seemingly clearer depiction of the original Gaussian pulse and the reflection by the absorbing boundary.

TLM and LGA simulations were performed for identical mesh sizes with slight differences implemented in the simulation of the TLM filter to accommodate its circular window. In general, the time domain waveforms obtained by the LGA experiments were found to be similar to the TLM data. However, the absorbing boundary proved to be only rudimentary for these transmission line experiments. It was possible however, to trace each reflection and transmission in the system knowing the sound speed in the LGA lattice, despite the $\mathrm{ABC}$ reflections propagating along the transmission lines of the filter..

Greater discrepancies hetween the two techniques were noted in the frequency domain. In particular, the level and frequency of the transmitted power differed from that obtained by the TLM result. A random frequency shift, as well as power level variation, were observed for individual simulations, indicating that the outcome is case dependent. Increasing the length of the filter main line, in an attempt to avoid the $\mathrm{ABC}$ reflections over the observed iterations, did not improve the result. 


\section{Chapter 6}

\section{CONCLUSIONS AND FUTURE RESEARCH}

\subsection{Conclusions}

Lattice gas automata consist of particles within a lattice that interact with their nearest neighbours according to simple deterministic rules. The algorithm evolves according to a two-step process, of collision and advection. Essential parts of the algorithm can be implemented in a few lines of code, while stability is maintained by the exact conservation of mass and momentum within the system, with no round-off errors. Boundary conditions are easily to implemented and can be simulated within a structure of significant complexity over a large number of time steps [12].

This thesis dealt with the application of lattice gas automata for use in modelling twodimensional homogeneous electromagnetic phenomena. The HPP-LGA technique has been used to successfully approximate the Navier-Stokes equation, and thus has been shown to simulate fluid flow. Similarities between fluid flow and wave propagation have suggested that LGA can be used to simulate electromagnetic models [2,9]. However, instead of explicitly solving the Navier-Stokes equations, the movements and interactions of thousands of gas particles are monitored [9]. The behaviour of the HPP lattice gas automaton has been shown to model conditions described by the two-dimensional linear wave equation. Thus, the simulation of two-dimensional, transverse-magnetic $\left(\mathrm{TM}_{\mathcal{z}}\right)$, or transverse-electric $\left(\mathrm{TE}_{\mathcal{z}}\right)$ wave propagation is possible with the application of appropriate boundary conditions and sources [2].

Properties inherent to two-dimensional HPP-LGA were detailed through simple twodimensional lattice gas experiments. Excitation in the LGA lattice was achieved using a 
Gaussian pulsed plane wave. Perturbation levels $20 \%$ above the background distribution of particles were applied to the mesh, due to a corruption of the signal in the lattice for higher amplitude levels. When the pulse peak was centered about a PMC boundary, a forward travelling Gaussian puised plane wave was initiated in the mesh. The PMC boundary models an open circuit termination for a $\mathrm{TM}_{2}$ wave propagation. Particles are simply bounced back into the lattice by a PMC boundary, undergoing change in the direction of their velocity. The $A B C$ was implemented for the simulation of a matched load termination. The $A B C$ "absorbed" incoming particles and reintroduced outgoing particles at a level of $\rho_{o}$ using a random number generator. A single column $\mathrm{ABC}$ was implemented throughout this thesis and was shown to have reflection coefficient of approximately $-15 \mathrm{~dB}$, in agreement with [8].

The effects of viscosity on the decay of the pulse perturbation over time and the sound speed in the HPP-LGA system were shown to be least significant for background particle densities of $0.4 \leq \rho_{0} \leq 0.5$. The sound speed in the lattice was found to slow for $\rho_{o}$ less than approximately 0.4 . The magnitude of the decay constant in (3.6) was determined to decreases with increasing $\rho_{o}$. For both the sound speed and pulse decay, these trends were reversed as the background density increased beyond approximately 0.5 .

The effects of spatial and ensemble averaging were briefly examined with respect to their influence on the inherent noise in the HPP-LGA system and the pulse dynamics, in both the time and frequency domain. Ensemble averaging was shown to decrease the rms noise level in the system by $\sqrt{N_{\text {ensemble }}}$, and had little effect on the frequency response of the incident pulse. However, increasing window size during spatial averaging had the effect of broadening and damping of the pulse. The filtering of the signal by the sampling window produced a time domain convolution of the perturbation signal with the sampling window. The Fourier transform is thus the product of the perturbation transform and the sinc function in the time domain. Thus, the larger the window, the wider the gate function in the time domain, and the smaller the perturbation bandwidth in the frequency domain. The noise level can be reduced by increasing the dimensions of the sampling window, but at the cost of reducing the bandwidth of the frequency response.

One of the goals of this thesis was to study the application of the HPP-LGA algorithm 
to scattering experiments. $A \mathrm{TM}_{2}$ cavity was modelled using a four walled PMC mesh. A single scattering object was placed in the mesh between two sampling windows. The forward and backward scattered waveforms were obtained by the two windows. Experiments were performed to further observe the effect of window size on waveforms of the $60 \Delta \times 120 \Delta$ scattering experiment, confirming the characteristics observed in Chapter 3. Additional experiments were performed where the cross-sectional area of the scattering object was varied. It was stated in Chapter 4, that the effects of the variations in the cylinder dimensions on the scattering pattern characteristics were not clearly apparent from the limited information obtained by the two sampling windows. However, the trends observed in LGA waveforms are almost identical to the TLM traces. The TLM method was used to assess the performance of the LGA data, as the implementation of the two algorithms in a a computer environment is similar. Furthermore, as an established computational technique, simulation results obtained using the TLM method represented an "exact" solution with which to evaluate the data obtained by the LGA algorithm.

A second important goal in this thesis was the modelling of a transmission line filter using the HPP-LGA algorithm. A simple single stub band reject filter was designed and simulated using both the TLM and LGA techniques. The absorbing boundary proved to be only rudimentary for these transmission line experiments. It was possible however, to trace each reflection and transmission in the system knowing the sound speed in the LGA lattice. In general, the time domain waveforms obtained by the LGA experiments were found to be similar to the TLM data, regardless of the ABC reflections propagating along the transmission lines of the filter.

The power levels in the frequency domain, in particular the transmitted power, are only adequate in comparison to the TLM result. It was shown that the ensemble averaging had some effect on the representation of the null at the design frequency. A random frequency shift, as well as power level variation, were observed for individual simulations, indicating that the outcome is case dependent. Simulations were performed for filters with a main line of longer length, in an attempt to avoid the $A B C$ reflections over the observed iterations. However, this did not prove successful in eliminating the frequency shift. 


\subsection{Future Research}

The results presented in this thesis indicated that the HPP-LGA method is a feasible technique for the analysis of two-dimensional electromagnetic propagation involving homogeneous systems. The accuracy of the method was appraised by comparing time and frequency domain waveforms to those computed by the transmission line matrix method, an established method of computation in electromagnetics. As the benefits of the HPP-LGA algorithm as a tool for computational electromagnetics have not been extensively explored, the possibilities for future research are quite considerable.

Improvements can be realized in the filter/transmission line modelling process by implementing absorbing boundary conditions with greater accuracy than that applied in this thesis. These improvements may involve a graded ABC [8], produced by creating a transition from free-space to the solid column of $A B C$ cells. The overall width of the $A B C$ is thus increased, with the density of $A B C$ cells for each column related to its relative position along the graded boundary. In addition, the random frequency shift observed by the reflected and transmitted power can be further examined with the implementation of an improved $\mathrm{ABC}$ boundary.

Improvements pertaining to the viscosity effects can be examined using the integer lattice gas techniques outlined in $[25,27,28]$ and applied to the experiments in this thesis. Three-dimensional vector wave behaviour could also be considered using a TLM-style HPP. LGA algorithm requiring up to 12 bits per cell $[41,42]$, or a FDTD-style implementation requiring 24 bits per cell [43]. This would initiate further study of the feasibility and differing characteristics of the altered method. This thesis has considered only twodimensional TM: scattering problems. The algorithm can be used to evaluate twodimensional $\mathrm{TE}_{\mathrm{z}}$ propagation.

It was mentioned in section 1.3 that LGA techniques can easily model complicated boundaries and simulate structures of significant complexity [12]. The ability of LGA to model small features in electromagnetic field problems was studied in [44], for analysis 
pertaining to curved conducting surfaces. A short, cylindrical perfectly conducting cavity, was implemented using stair-stepping discretization. The extremely fine spatial discretization required by the LGA mesh achieved an accurate description of the boundary with minimal stair-stepping errors. Experiments with more intricate scattering objects (e.g. a true cylinder, rather than a rectangular approximation), or more detailed mesh geometries, may therefore be pursued to improve upon the coarser, and relatively simple rectangular mesh. In addition, regarding the scattering experiments in this thesis, a comprehensive study of the scattering patterns may also be carried out, by placing additional sampling windows in the entire region surrounding the scattering object.

More complicated media can be modelled by adjusting the rules within the mesh. Inhomogeneous systems can be realized using the theory of rest particles at a lattice site, addressed in $[2,8,11]$. The addition of rest particles acts to store mass (energy) at a cell for a short period of time. This has the effect of decreasing the average velocity of moving particles. Therefore, the propagation speed of the small density perturbations in the mesh is reduced, and correspondingly, the dielectric constant is increased. This theory of rest particles ca be applied to simulate more complex scattering in inhomogeneous media. 


\section{Appendix}

\section{COMPUTATIONAL EFFICIENCY}

It was stated in section 1.3 that LGA algorithms are inherently parallel, evolving more efficientiy on parallel computing machines. The lattice gas machine, known as the CAM-8, can run simulations at a rate of about 3 billion cell updates per second, on spaces of up to half a billion cells with a single bit per cell; with 16 bits per cell, simulations run at about 200 million site updates per second on spaces of up to 32 million cells [8]. The CAM-8 is comprised of $64 \mathrm{MB}$ of DRAM, where the lattice data is located. The look-up table is resident in the $2 \mathrm{MB}$ SRAM.

The experiments in this thesis were performed in a Unix environment, usually by an Uitra 5 or Ultra 10 station. The amount of CPU time occupied by a particular experiment is listed in Table A.1, where the number of operations within the mesh is approximately equivalent to,

$$
4-N_{x} N_{y} \text { (Number of iterations) }
$$

where $N_{x}$ and $N_{y}$ are the number of cells in $x$ and $y$ within the mesh. In each case, the number of operations is on the order of $10^{9}$. The mesh areas for the cases listed in Table A.1 range from 71781 cells in the single transmission line case to 641601 cells for the scattering experiments. In addition to the lattice update, computational time must also be allotted to the computation of the macroscopic observables. Depending on mesh size and the number of iterations in each experiment, the simulations outlined in Table A.l took from half an hour to four hours to complete by an Ultra 10. These would be completed in a matter of seconds in the CAM-8 environment, ranging from 2.87 seconds for the single transmission line and 54.74 seconds for the filter. 
Table A.1: Number of operations and CPU time completed for selected experiments on Unix Ultra 5 and Ultra 10 stations.

\begin{tabular}{|c|c|c|c|}
\hline Experiment & Unix station & $\begin{array}{c}\text { CPU time } \\
\text { (hrs:min:sec) }\end{array}$ & $\begin{array}{c}\text { Number of } \\
\text { operations }\end{array}$ \\
\hline $\begin{array}{c}\text { PMC experiments } \\
\text { (Section 3.2.1) }\end{array}$ & Ultra 5 & $1: 22: 11$ & \multirow{2}{*}{$2.569 \times 10^{9}$} \\
\cline { 2 - 3 } & Ultra 10 & $0: 52: 59$ & \\
\hline $\begin{array}{c}\text { Scattering experiments } \\
\text { (Chapter 4) }\end{array}$ & Ultra 5 & $1: 53: 42$ & \multirow{2}{*}{$4.106 \times 10^{9}$} \\
\cline { 2 - 3 } & Ultra 10 & $1: 22: 39$ & \\
\hline \multirow{2}{*}{$\begin{array}{c}\text { Single transmission } \\
\text { line (Section 5.2.) }\end{array}$} & Ultra 5 & $0: 43: 27$ & \multirow{2}{*}{$0.574 \times 10^{9}$} \\
\cline { 2 - 3 } & Ultra 10 & $0: 28: 20$ & \multirow{2}{*}{$11.748 \times 10^{9}$} \\
\hline \multirow{2}{*}{$\begin{array}{c}\text { Filter experiments } \\
\text { (Section 5.3) }\end{array}$} & Ultra 5 & $5: 33: 52$ & \\
\cline { 2 - 3 } & Ultra 10 & $3: 53: 17$ & \multicolumn{2}{|c}{} \\
\hline
\end{tabular}




\section{REFERENCES}

[1]. Toffoli, T. and N. Margolis, Cellular Automata Machines. Cambridge: The MIT Press, 1987.

[2]. Simons, N. R., M. Cuhaci, N. Adnani, and G. Bridges, "On the Potential Use of Cellular Automata machines for Electromagnetic Field Solution", International Journal of Numerical Modelling: Electronic Networks, Devices and Fields, 8, 301$312(1995)$.

[3]. Wolfram, S., Cellular Automata and Complexity - Collected Papers. Reading, MA: Addison-Wesley, 1994.

[4]. Toffoli, T., "Cellular Automata as an Alternative to (Rather than an approximation of) Differential Equations in Modeling Physics", Physica D, 10, 117-127 (1984).

[5]. Hardy, J., O. de Pazzis, Y. Pomeau, "Molecular dynamics of a classical lattice gas: Transport properties and time correlation functions", Physical Review A, 13, 19491961 (1976).

[6]. Frisch, U., B. Hasslacher, and Y. Pomeau, "Lattice-Gas Automata for the NavierStokes equation", Physical Review Letters, 56, 1505-1508 (1986).

[7]. Adnani, N., "Cellular Automata Models for the Two-Dimensional Scalar Wave Equation", M. Sc. Thesis, University of Manitoba (1996).

[8]. Cule, D., "HPP Lattice-Gas Automata for Computational Electromagnetics", M. Sc. Thesis, University of Manitoba (1998). 
[9]. Bornholdt, J. M., and K. D. Tatalias, "The application of lattice gas techniques to electromagnetic problems", IEEE Transactions on Magnetics, 25, 2916-2918 (1989).

[10]. Simons, N. R., G. E. Bridges, B. W. Podaima, A. R. Sebak, "Cellular Automata as an Environment for Simulating Electromagnetic Phenomena", IEEE Microwave and Guided Wave Letters, 4, 247-249 (1994).

[11]. Adnani, N., M. Zhang, D. Cule, N. Simons, G. Bridges, and M. Cuhaci, "Modeling wave mechanics using lattice gas automata", in Proceedings of Symposium on Antenna Technology and Applied Electromagnetics, 203-210, Montreal, August 1996.

[12]. Shimomura, T., G. Doolen, B. Hasslacher, and C. Fu, "Calculations using lattice gas techniques", in Los Alamos Science, Special issue, $201-210$ (1987).

[13]. Toffoli, T., "CAM: A High Performance Cellular-Automaton Machine", Physica 10D, 195-204 (1984).

[14]. Hardy, J., Y. Pomeau, and O. de Pazzis, "Time evolution of a two-dimensional model system: Invariant states and time correlation functions", Journal of Mathematical Physics, 14, 1746-1755 (1973).

[15]. Frisch, U., D. d'Humieres, B. Hasslacher, P. Lallemand, Y. Pomeau, J.P. Rivet, "Lattice gas hydrodynamics in two and three dimensions", Complex Systems, 1, 649707, (1987).

[16]. Wolfram, S., "Cellular automata fluids I: Basic theory", Journal of Statistical Physics, 45, 471-526 (1986). 
[17]. Frisch, Hasslacher, and Y. Pomeau, "Lattice gas automata for the Navier-Stokes equation", Physica D, 49, 6920-6926 (1994).

[18]. Thiele, L., "Parallel implementation of cellular systems for numerical modeling", International Journal of Numerical Modelling: Electronic Networks, Devices and Fields, 5, 203-218 (1992).

[19]. Paterson, A., A First Course in Fluid Dynamics. Cambridge: Cambridge University Press, 1983.

[20]. Batchelor, G., An Introduction to Fluid Dynamics. Cambridge: Cambridge University Press, 1967.

[21]. Chen, H., S. Chen, G. Doolen, and Y. Lee, "Simple lattice gas models for waves", Complex Systems, 2, 259-267 (1988).

[22]. Boghosian, B., and C. Levermore, “A cellular automaton for Burger's equation", Complex Systems, 1, 17-30 (1987).

[23]. Simons, N, "Development and Application of Differential-Equation Based Numerical Techniques to Electromagnetic Scattering and radiation Problems", Ph.D Thesis, University of Manitoba (1994).

[24]. Ingard, K. U., Chapter 12 in Fundamentals of Waves and Oscillations. Cambridge: Cambridge University Press, 1988.

[25]. Bridges, G., and N. Simons, "Multi-Bit Lattice Gas Automaton for Computational Electromagnetics", in Program and Abstracts of North American Radio Science Meeting (URSI), 266, Montreal, August (1997). 
[26]. Boghosian, B., J. Yepez, F. Alexander, and N. Margolus, "Integer Lattice Gases", Physical Review E, 55, 4137-4147 (1997).

[27]. Bridges, G., and N. Simons, "Extremely Low-Precision Integer Cellular Array Algorithm for Computational Electromagnetics", IEEE Microwave and Guided Wave Letters, 9, 1-3 (1999).

[28]. Treurniet, J., N. Simons, G. Bridges, "Integer Lattice Gas Automata for Computational Electromagnetics", IEEE Transactions on Microwave Theory and Techniques, 48, 985-990 (2000).

[29]. Poularikas, A., and S. Seely, Signals and systems. Boston, MA: PWS Publishers, 1985.

[30]. Lathi, B., Communication Systems. New York, NY: John Wiley and Sons Inc., 1968.

[31]. Feynman, R., R. Leighton, and M. Sands, The Feynman Lectures on Physics. Reading, MA: Addison-Wesley Publishing Company Inc., 1963.

[32]. Johns, P., and R. Beurle. "Numerical Solution of two-dimensional Scattering Problems using a Transmission Line Matrix", Proceedings of Institute of Electrical Engineers, 118, 1203-1208 (1971).

[33]. Simons, N., "Application of the Transmission-Line Matrix Method to Open Region Field Problems", M. Sc. Thesis, University of Manitoba (1989).

[34]. Christopoulos, C., Transmission-Line Modelling Method: TLM. New York: IEEE Press, 1995. 
[35]. Hoefer, W., MEFiSTo-2D Electromagnetic Simulator: The MEFiSTo-2D Theory. Victoria, BC: Faustus Scientific Corporation, 1998.

[36]. Hoefer, W., "The Transmission-Line Matrix Method: Theory and Applications", IEEE Transactions on Microwave Theory and Techniques, 33, 882-893 (1985).

[37]. Bracewell, R., The Fourier Transform and its Applications. New York, NY: McGraw-Hill Inc., 1965.

[38]. Silver, S., Microwave Antenna Theory and Design. New York, NY: Dover Publications Inc., 1965.

[39]. Bowman, J., T. Senior, and P. Uslenghi, eds., Electromagnetic and Acoustic Scattering by Simple Shapes. New York, NY: John Wiley and Sons Inc., 1969.

[40]. Matthaei, G., L. Young, and E. Jones, Microwave Filters, Impedance-Matching Networks and Coupling Structures. New York, NY: McGraw-Hill Inc., 1964.

[41]. Zhang, M., G. Bridges, and N. Simons, "A 3D Vector Model for Computational Electromagnetics", in Symposium on Antenna Technology and Applied Electromagnetics, Ottawa, Ontario, August 1998.

[42]. Simons, N., G. Bridges, M. Cuhaci, "A Lattice Gas Automaton Capable of Modeling Three-Dimensional Electromagnetic Fields", Journal of Computational Physics, 151, 816-835 (1999).

[43]. Simons, N., "Application of Lattice Gas Automata to the Solution of Electromagnetic Filed Problems", in $6^{\text {th }}$ International Conference on Discrete Models for Fluid Dynamics, Boston, Massachusetts, August 1996. 
[44]. Cule, D., N. Simons, G. Bridges, M. Cuhaci, and J. Lovetri, "Investigation of Geometrically Small Features within Numerical Solutions to Electromagnetic Filed Problems", in Symposium on Antenna Technology and Applied Electromagnetics, Montreal, Quebec, August 1996.

[45]. Cheng, D., Field and Wave Electromagnetics. Reading, MA: Addison-Wesley Inc., 1989.

[46]. Oppenheim, A. and A. Willsky, Signals and Systems. Englewood Cliffs, NJ: Prentice-Hall Inc., 1983.

[47]. Doolen, G. D. et al., Ed., Lattice Gas Methods for Partial Differential Equations. Reading, MA: Addison-Wesley, 1990.

[48]. Chopard, B., and M., Droz, Cellular Automata Modeling of Physical Systems. Cambridge, UK: Cambridge University Press, 1998.

[49]. Lawniczak, A., and R. Kapral, Ed., Pattern Formation and Lattice Gas Automata. Providence, RI: American Mathematical Society, 1996. 